\title{
Regulation of H2B monoubiquitination pathway in breast cancer
}

\author{
Ph.D. Thesis \\ for the award of the degree \\ "Doctor of Philosophy (Ph.D)" \\ of the Georg-August-Universität Göttingen \\ within the doctoral program Molecular Biology of Cells \\ of the Georg-August University School of Science (GAUSS) \\ Submitted by \\ Upasana Bedi \\ born in \\ New Delhi, India
}

Göttingen, 2013 


\section{Thesis Supervisor:}

Prof. Dr. Steven A. Johnsen

\section{Members of the Thesis Committee:}

Prof. Dr. Steven A. Johnsen (Reviewer)

Dept. of Tumor Biology,

University Medical Center Hamburg Eppendorf, Hamburg

Prof. Dr. Holger Reichardt (Reviewer)

Dept. of Cellular and Molecular Immunology

Göttingen University Medical School, Göttingen

Prof. Dr. Gregor Bucher

Dept. of Developmental Biology

Georg-August-University, Göttingen

Date of oral examination: 24th January'2014 


\section{Affidavit}

I hereby declare that the PhD thesis entitled "Regulation of $\mathrm{H} 2 \mathrm{~B}$ monoubiquitination in breast cancer" has been written independently and with no other sources and aids than quoted.

\section{Upasana Bedi}

December, 2013

Göttingen 


\section{$\underline{\text { Table of Contents }}$}

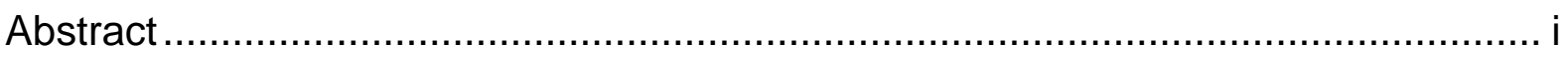

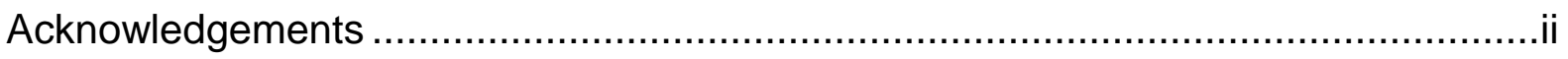

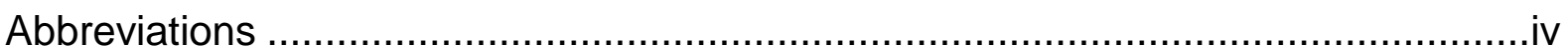

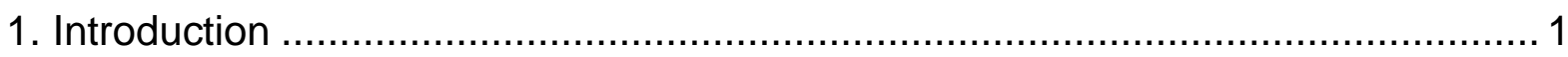

1.1 Chromatin organization and histone modifications...................................... 1

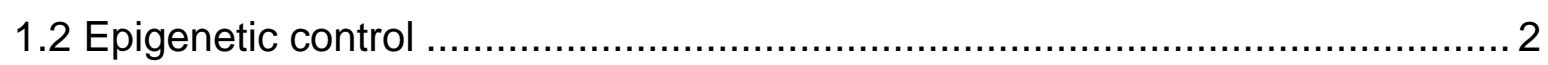

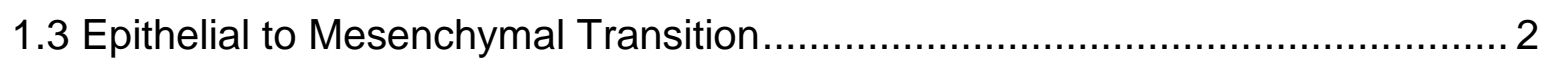

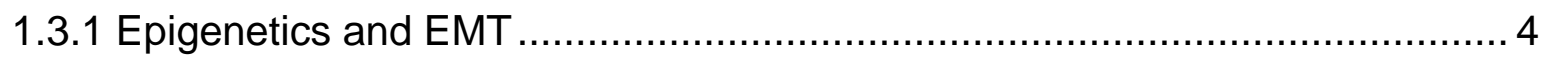

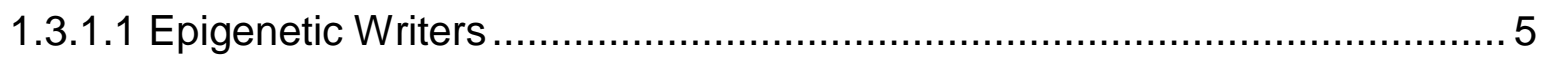

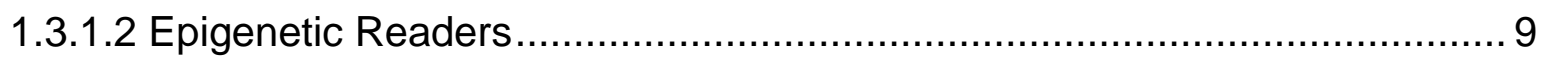

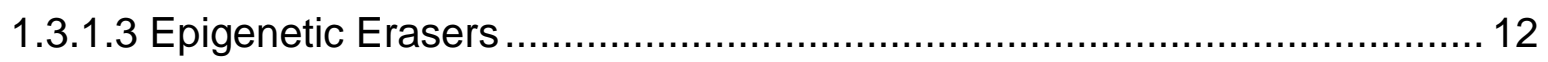

1.3.2 Epigenetic Regulation of EMT inducing Transcription factors .................... 14

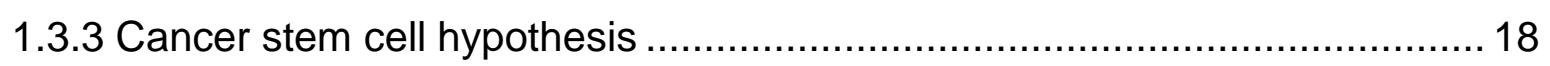

1.4 Estrogen signaling and Estrogen Receptor............................................ 20

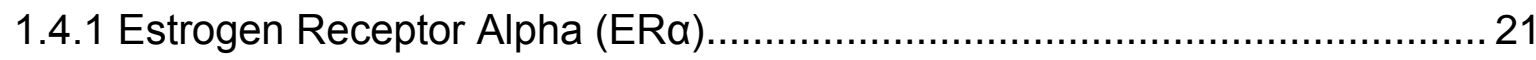

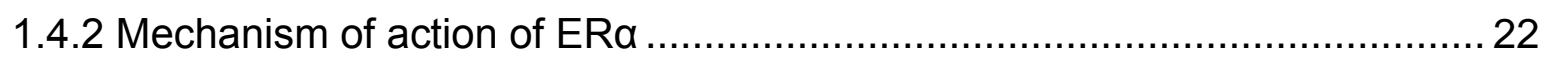

1.5 Histone H2B monoubiquitination (H2Bub1) ............................................. 23

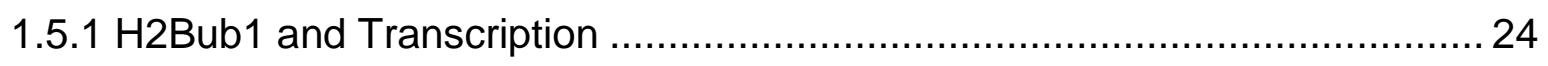

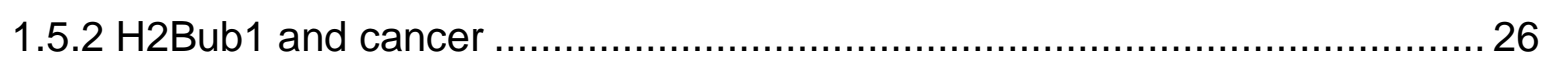

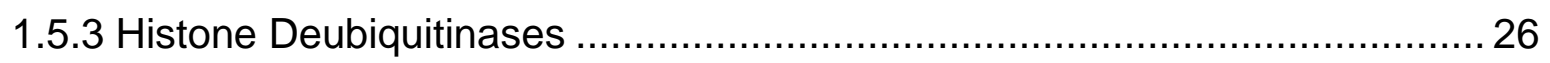

1.6 Upstream regulation of H2Bub1 and transcription ................................... 28

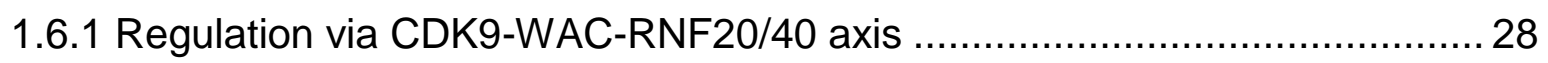

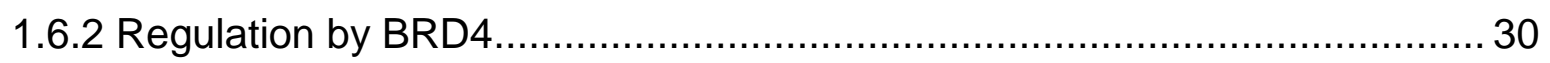

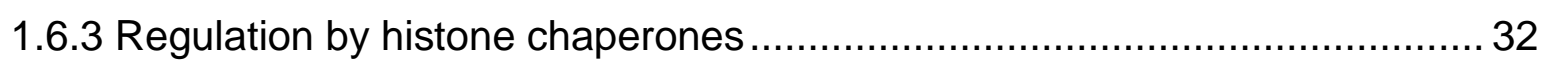

1.6.3.1 Yeast SPT6 (Suppressor of Ty 6) ………............................................ 33

1.6.3.2 SUPT6H (Human homolog of yeast - Suppressor of Ty 6) ....................... 33

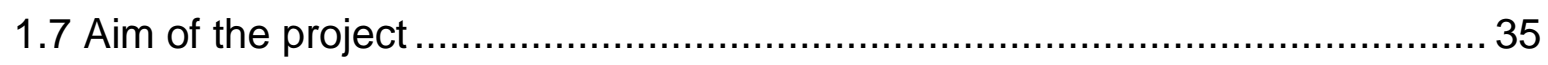

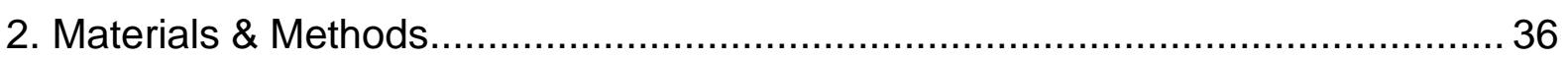

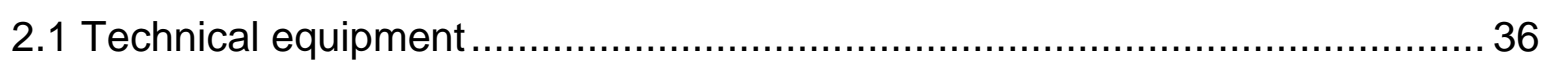

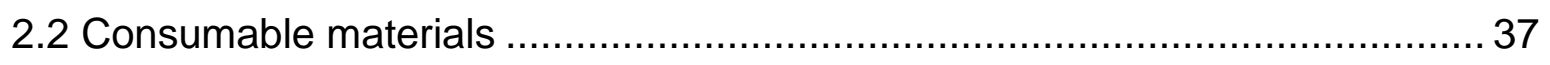

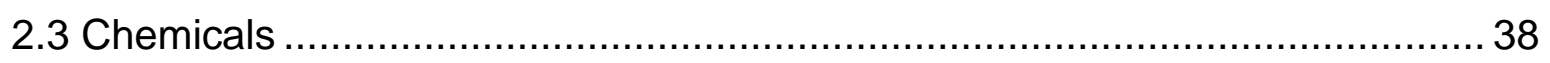

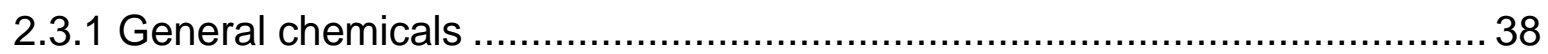




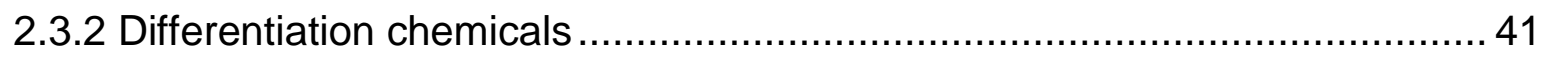

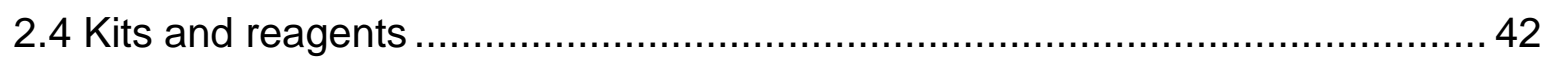

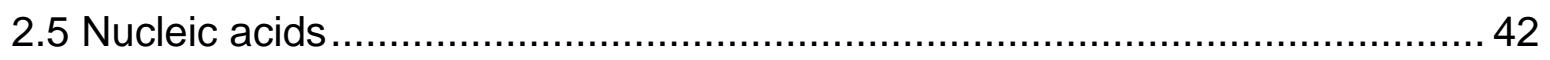

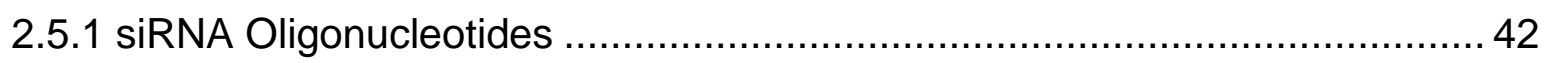

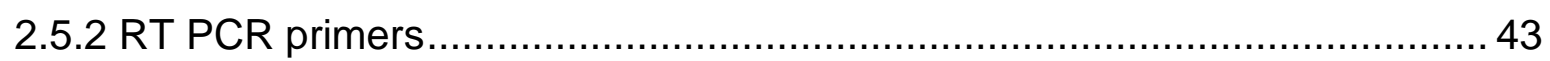

2.5.2.1 Quantitative PCR primers (qPCR) ….............................................. 43

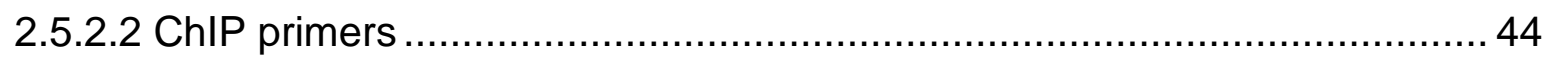

2.5.2.3 Primers for ChIP-seq Library preparation.............................................. 45

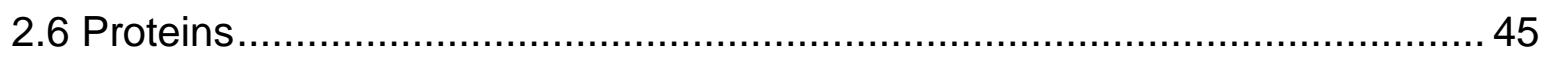

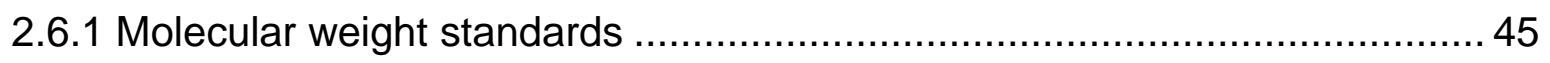

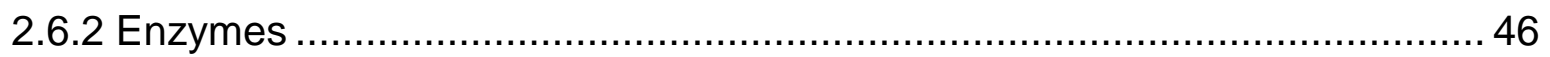

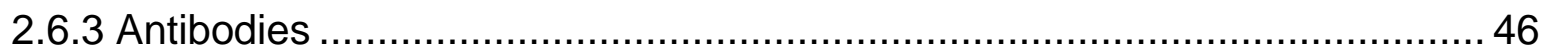

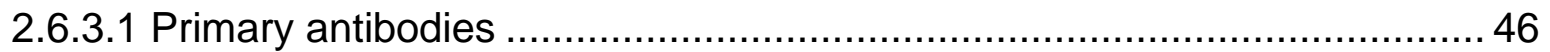

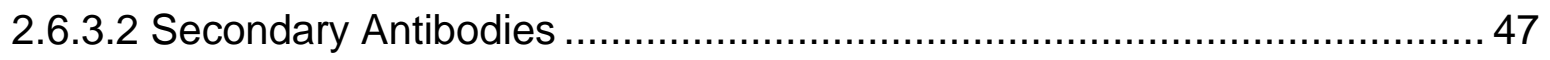

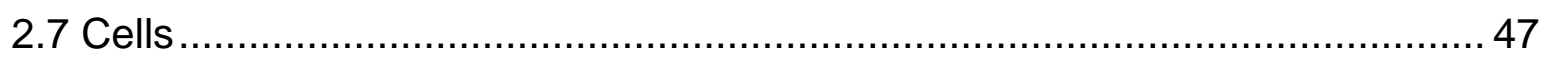

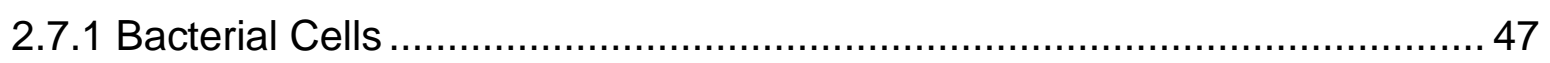

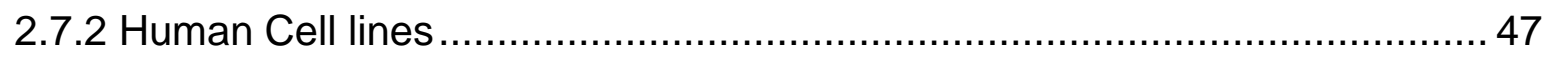

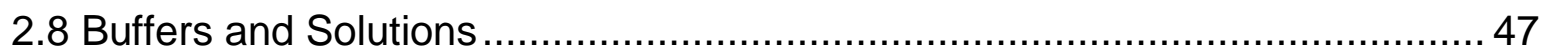

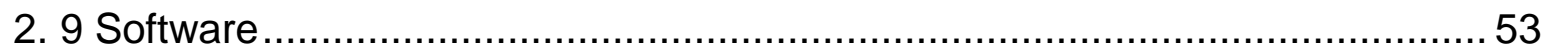

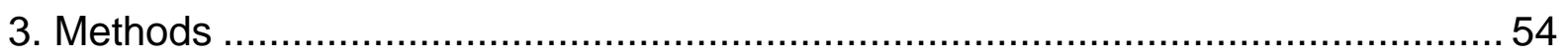

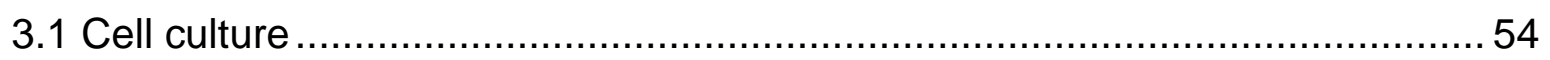

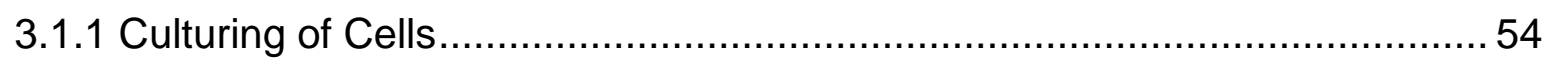

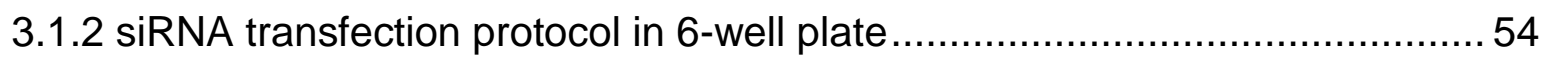

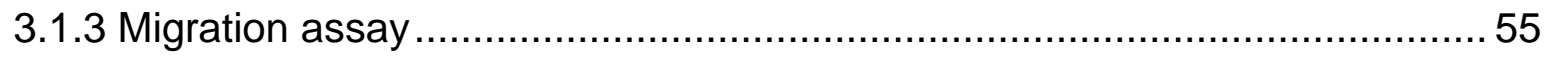

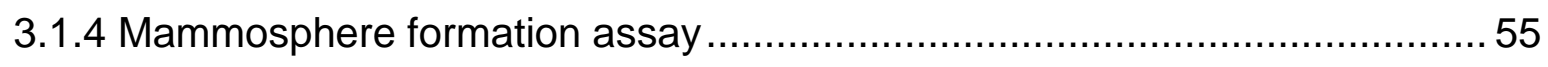

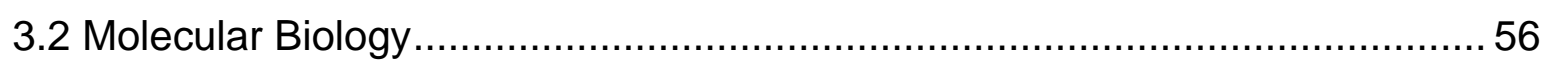

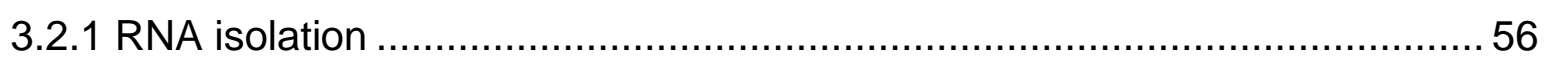

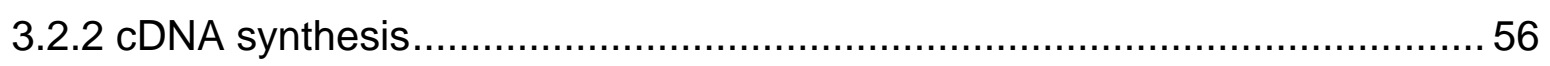

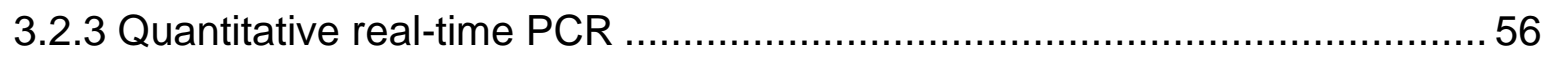

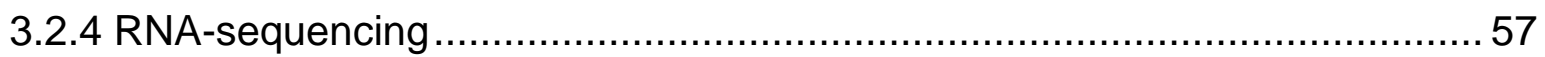

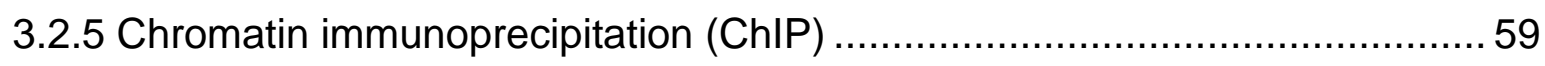

3.2.6 Chromatin immunoprecipitation-sequencing (ChIP-seq) ............................ 60

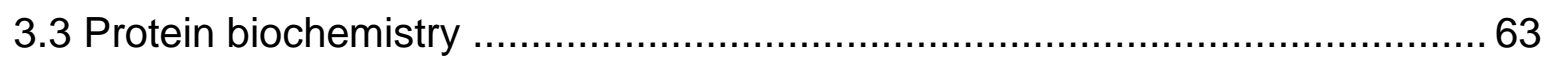




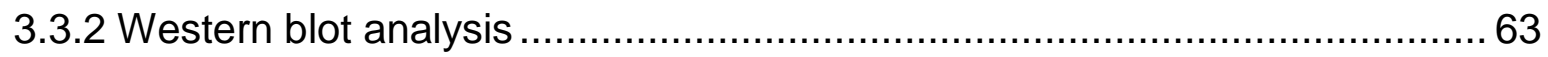

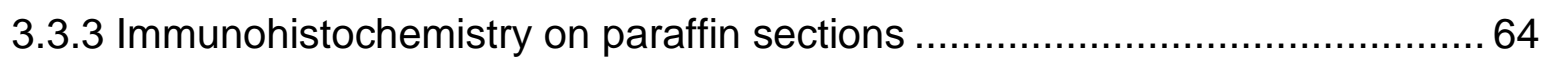

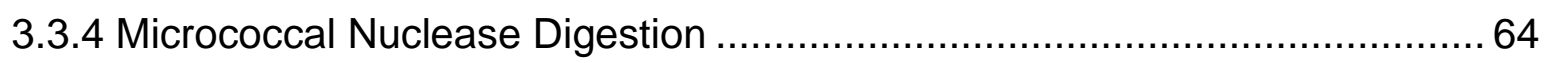

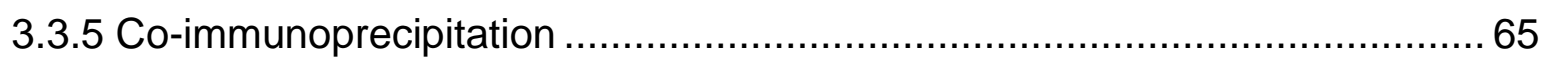

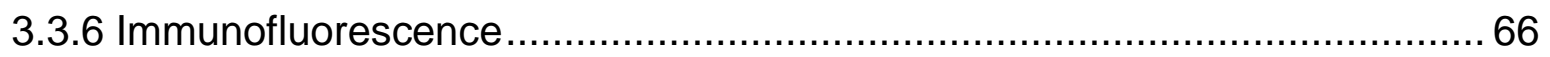

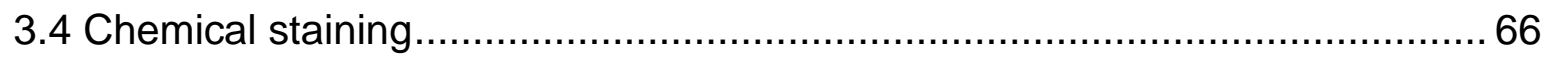

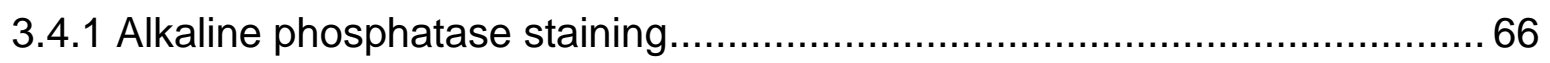

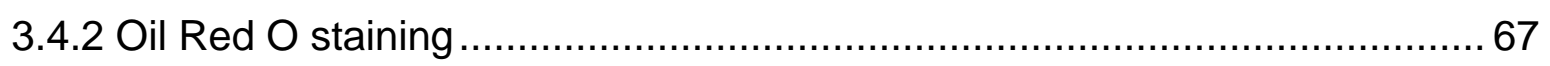

3.4.3 Carmine alum staining for mammary ducts .............................................. 67

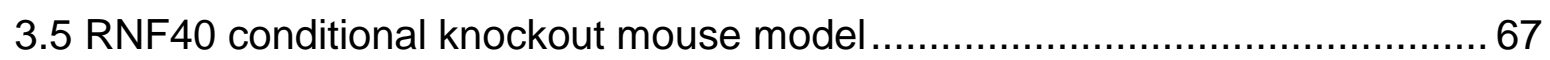

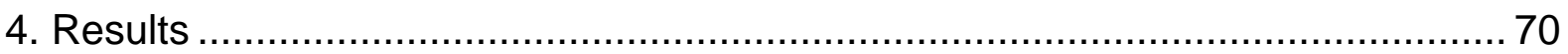

4.1 Estrogen signaling and dedifferentiation ................................................ 70

4.1.1 SUPT6H is required for ERa activity ................................................... 71

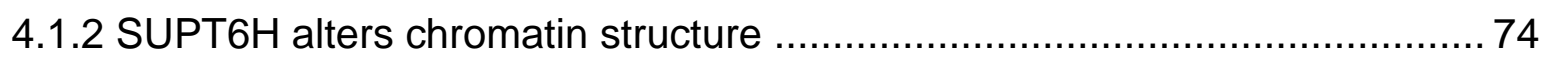

4.1.3 SUPT6H is required for maintaining $\mathrm{H} 2 \mathrm{~B}$ monoubiquitination .................... 76

4.1.4 SUPT6H expression and H2Bub1 levels are inversely correlated with breast

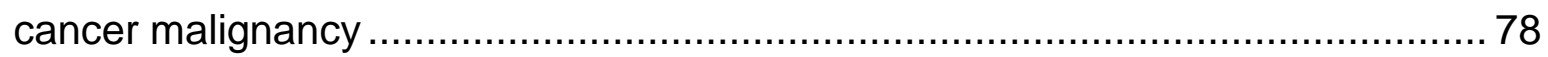

4.1.5 SUPT6H is required for cellular differentiation ....................................... 83

4.1.6 SUPT6H suppresses H3K27me3 on ERa- and differentiation-regulated

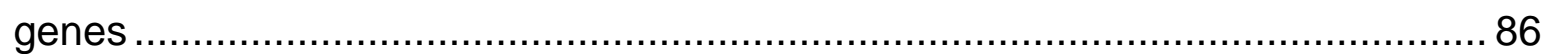

4.2 H2Bub1 and Mammary Stem Cell Phenotype ........................................... 88

4.2.1 RNA-sequencing identifies similarities in gene expression profiles following

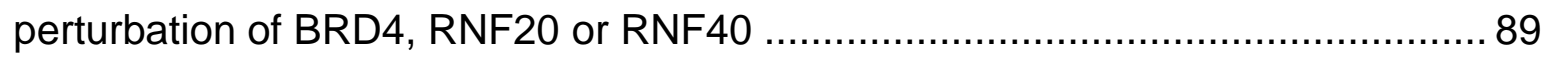

4.2.2 Overlap between BRD4 and H2Bub1 recruitment sites ........................... 92

4.2.3 RNA Sequencing Reveals Regulation of Mammary Stem Cell Gene

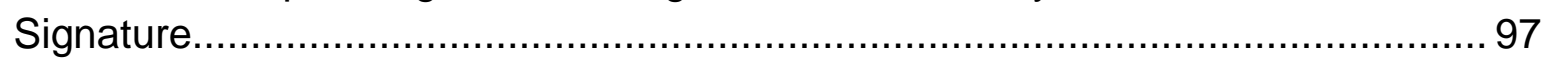

4.2.4 Loss of BRD4 and H2Bub1 is coupled with EMT phenotype ................... 102

4.2.5 Conditional RNF40 knock-out mice indicates enhanced mammary gland

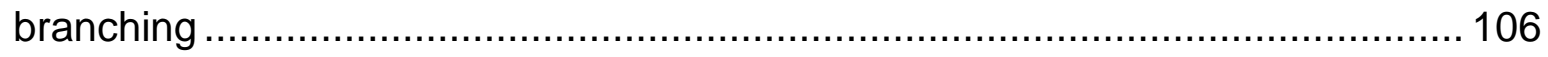

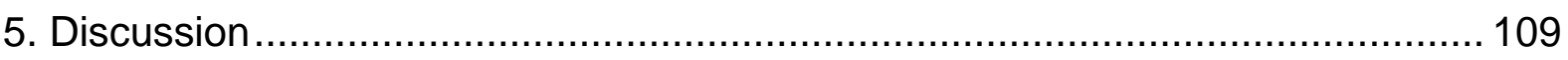

5.1 Histone chaperone SUPT6H interacts with H2Bub1 for active transcription . 109

5.2 BRD4 and H2Bub1 act together during transcription .................................. 112

5.3 BRD4 and H2Bub1 "teamwork" suppresses EMT and mammary stem cell phenotype 
5.4 Possible mechanisms of regulation by BRD4 and H2Bub1 115

5.4.1 Gene Bivalency 115

5.4.2 Repressive role of BRD4 and H2Bub1 ............................................. 117

5.4.3 Regulation of tumor suppressor microRNAs ……................................... 117

5.4.4 Role of BRD4 and H2Bub1 in regulation of enhancer RNA expression ..... 119

5.4.5 Loss of H2Bub1 mimics activation of deubiquitinases (DUBs) .................. 119

5.5 BRD4 inhibition by JQ1 enriches cancer stem cell pool .............................. 120

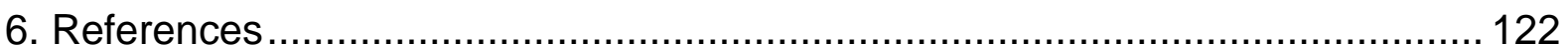

7. Curriculum Vitae......................................................Error! Bookmark not defined. 


\section{Abstract}

H2B monoubiquitination (H2Bub1) regulation via CDK9-WAC-RNF20/40 axis has been well illustrated. It is interesting to determine the upstream regulators which dictate the process of monoubiquitination of $\mathrm{H} 2 \mathrm{~B}$. On further investigation, histone chaperone SUPT6H known to bind P-Ser2 RNAPII was observed to regulate ERadependent signaling through H2Bub1 pathway. Perturbation of SUPT6H led to a decrease in H2Bub1 resulting in impaired estrogen-dependent signaling and mesenchymal stem cell differentiation due to increase in the H3K27me3 repressive mark on the promoters of the genes. Moreover, SUPT6H levels were decreased with tumor progression. Together, these data identify SUPT6H as a new epigenetic regulator of ERa activity and cellular differentiation.

Further upstream regulator of CDK9, BRD4 was also examined. For the first time, we showed the connection between BRD4 and H2Bub1 pathway. They both regulated the gene expression in a similar fashion and had gene expression-dependent occupancy on the genes. Interruption of this pathway by BRD4 or H2Bub1 depletion resulted in acquisition of EMT and stem cell-like phenotype in mammary epithelial cells. The preliminary data for conditional RNF40 KO in mammary gland also showed increased mammary branching further emphasizing the critical role of H2Bub1 as a tumor suppressor. These important findings could help to harness these epigenetic regulators for anti-cancer therapy. 


\section{Acknowledgements}

I would like to express sincere gratitude to my supervisor, Prof. Steven A. Johnsen for his continuous support and enlightenment. He has been the pillar of strength and kept motivating despite several unsuccessful experiments and submissions. He is extremely liberal and allowed me to pursue the project I wanted to. He has been always available for any kind of help and kept us all happy with his vivacity. I could not have imagined a professor being so supportive and fun-loving in the lab. One could not wish to have a friendlier supervisor.

I would like to thank Prof. Hans K. Will for his motivation. He is a great human being and a wonderful advisor.

I would like to thank my thesis committee members, Prof. Holger Reichardt and Prof. Gregor Bucher for their encouragement, insightful comments and thoughtful discussions.

I would sincerely thank Prof. Matthias Dobbelstein, present and former members of Molecular Oncology, Göttingen who welcomed me and made it a pleasure to work with them. I would also thank Prof. Klaus Pantel and members of Institute of Tumor Biology, UKE for a warm welcome after moving to Hamburg.

I would also like to thank all present and former members of AG Johnsen. It has been a fun-filled journey working in the lab despite such terrible days of failures. I would really like to thank Zeynab Najafova and Simon Baumgart for relaxing coffee break discussions. I would like to thank former members, Judith Pirngruber, Tanja Prenzel, Andrei Shchebet, Oleksandra Karpiuk, Magali Hennion and Yvonne BegusNahrmann for a wonderful time and helping me learn things in the lab. I would like to 
thank Theresa Gorsler, Vijayalakshmi Kari, Sankari Nagarajan, Vivek Mishra, Sanjay Raul, Tareq Hossan, Anusha Thota and Wanhua Xie for conducive working atmosphere.

I would also thank Julia Spotter and Dr. Florian Wegwitz for their tremendous help for the mice experiments.

Last but not the least, I would like to thank my parents, Anup Bedi and Neeru Bedi for inculcating all the values I have and making me what I am. I would like to thank my brother, Kunal for all the love and support. I would thank my future father-in law, Rohit Chanana for always believing in me and motivating me when I gave up. A big thank you to my life partner, Rohan Chanana for being there and letting me vent out all the frustrations I had after scientific failures. I would like to thank all my friends in Göttingen who made this journey memorable. You all would remain in my heart forever.

I would not have completed this work without you all being part of my life. Thank you everyone! 


\section{Abbreviations}

A

AF

APS

BGP

BMI-1

BGS

BRD4

Bre1

BSA

${ }^{\circ} \mathrm{C}$

CDK7

CDK9

CDNA

ChIP

ChIP-seq

$\mathrm{CO}_{2}$

COMPASS

CpG

CSS

CTD

CXCL12

DAPI

DEPC

DMEM

DMSO

DNA

dNTP

DRB

DSIF

DTT

DUB
Alpha

Transactivation function

Ammonium persulfate

B-Glycerophosphate

$B$ lymphoma Mo-MLV insertion region 1 homolog

Bovine Growth Serum

Bromodomain containing 4

BREfeldin A sensitivity

Bovine serum albumin

degree Celsius / centrigrade

Cyclin-Dependent Kinase 7

Cyclin-Dependent Kinase 9

Complementary DNA

Chromatin immunoprecipitation

ChIP followed by high-throughput sequencing

Carbon dioxide

Complex proteins associated with Set $1 p$

Cytosine phosphate Guanine

Charcoal-dextran treated FBS

Carboxy-terminal Domain

Chemokine (C-X-C motif) ligand 12

4',6-diamidino-2-phenylindole

Diethylpyrocarbonate

Dulbecco/Vogt modified Eagle's minimal essential medium

Dimethyl sulfoxide

Deoxyribonucleic acid

deoxyribonucleotide

5,6-dichloro-1-beta-D-

ribofuranosylbenzimidazole

DRB Sensitivity Inducing Factor

Dithiothreitol

Deubiquitinating enzyme 
E2

E1 enzyme

E2 enzyme

E3 enzyme

EDTA

e.g.

EGF

EMT

ENY2

ER

ERE

et al.

$\mathrm{EtOH}$

FACT

FDR

GCN5

GREB1

$\mathrm{h}$

$\mathrm{H} 2 \mathrm{~A}$

H2Aub1

$\mathrm{H} 2 \mathrm{~B}$

H2Bub1

$\mathrm{H} 3$

H3K4me3

H3K27me3

H3K36me3

H3K79me3

$\mathrm{H} 4$

HAT

hMSCs

HMT
17ß-Estradiol

Ubiquitin-activating enzyme

Ubiquitin-conjugating enzyme

Ubiquitin-ligase

Ethylenediaminetetraacetic acid

exempli gratia $=$ for example

Epidermal growth factor

Epithelial-mesenchymal transition

Enhancer of yellow 2 homolog

Estrogen Receptor

Estrogen Responsive Element

et alii $=$ and others

Ethanol

Facilitates Active Chromatin

Transcription

False Discovery Rate

Histone asetyltransferase, general control of amino acid synthesis protein 5 Growth regulation by estrogen in breast cancer 1

Hour

Histone 2A

Monoubiquitinated histone $2 \mathrm{~A}$

Histone 2B

Monoubiquitinated histone $2 \mathrm{~B}$

Histone 3

Histone 3 trimethylated at position lysine 4

Histone 3 trimethylated at position lysine 27

Histone 3 trimethylated at position lysine 36

Histone 3 trimethylated at position lysine 79

Histone 4

Histone acetyltransferase

Human Mesenchymal Stem Cells

Histone methyltransferase 
hnRNPK

HRP

HSC70

IAA

IBMX

$\lg G$

$\mathrm{kDa}$

$\mathrm{m}$

$\mu$

MEM

$\min$

MLL

mRNA

$\mathrm{n}$

NELF

NEM

NP-40

PBS

PBS-T

PCR

PDK4

PGR

$\mathrm{pH}$

PPARY

P-TEFb

qPCR

Rad6

RASD1

RING

RNA

RNAPII
Heterogeneous Nuclear

Ribonucleoprotein $\mathrm{K}$

Horseradish peroxidise

Heat shock $70 \mathrm{kDa}$ protein

lodacetamide

Isobutyl-methyl-xanthine

Immunoglobulin G

kilo Dalton

milli $\left(10^{-3}\right)$

micro $\left(10^{-6}\right)$

Minimum Essential Media

Minute

Myeloid/lymphoid or mixed-lineage

leukemia

messenger RNA

nano $\left(10^{-9}\right)$

Negative elongation factor

$\mathrm{N}$-ethylmaleimide

Nonidet P40

Phosphate Buffered Saline

Phosphate Buffered Saline with Tween-

20

Polymerase Chain Reaction

Pyruvate Dehydrogenase Kinase, isozyme 4

Progesterone receptor

Measurement of acidity or alkalinity of a solution

Peroxisome Proliferator-Activated

Receptor gamma

Positive Transcription Elongation Factor beta

Quantitative real-time PCR

Radiation sensitivity protein 6

RAS, dexamethasone-induced 1

Really Interesting New Gene

Ribonucleic acid

RNA Polymerase II 
RNF20

RNF40

RT

RT-PCR

SAGA

SDS

SDS-PAGE

sec

siRNA

SUPT6H

Taq

TEMED

TFF1

Tris

U

UBE2A

USP22

$\mathrm{V}$

WAC

WB
Ring finger protein 20

Ring finger protein 40

Room Temperature

Reverse Transcription PCR

Spt-Ada-Gcn5-Acetyltransferase

Sodium dodecylsulfate

Sodium dodecylsulfate polyacrylamide gel electrophoresis

Second

Small interfering RNA

Supressor of Ty Homologue- 6

Thermus aquaticus

Tetramethylethylenediamine

Trefoil factor 1

Tris(hydroxymethyl)aminomethane

Unit (enzyme activity)

Ubiquitin-conjugating Enzyme E2A

Ubiquitin Specific Peptidase 22

Voltage

WW domain containing adaptor with coiled-coil

Western blot 


\section{List of Figures}

Fig. 1. The process of EMT and its reversible MET .....................................................

Fig. 2. SUPT16H, FACT subunit correlates with the epithelial phenotype in human

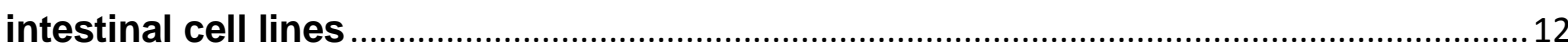

Fig. 3. EMT-TFs interact with epigenetic regulators to repress epithelial genes............16

Fig. 4. Signaling activators induce transcription of EMT-TFs which in turn regulate

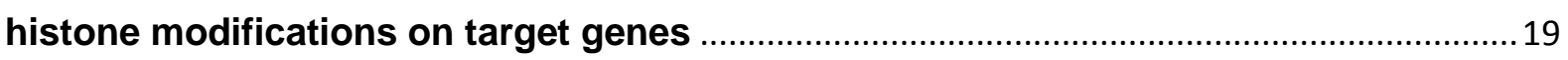

Fig. 5. The regulation of H2Bub1 through CDK9-WAC-RNF20/40 axis .............................29

Fig. 6. Detailed graphical overview for the Rnf40 conditional knockout mouse

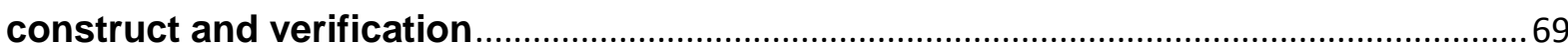

Fig. 7. SUPT6H knockdown decreases estrogen-induced gene expression ................... 72

Fig. 8. Effect of single siRNA SUPT6H knockdown on estrogen-induced gene expression

Fig. 9. Decreased ERa and SUPT6H recruitment to distal EREs of GREB1 and PGR upon SUPT6H knockdown

Fig. 10. Changes in chromatin structure upon SUPT6H knockdown as determined by

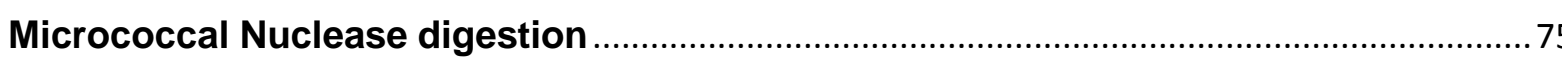

Fig. 11. SUPT6H knockdown decreases RNF40 protein levels and H2Bub1 .................. 77

Fig. 12. Decreased H2Bub1 on transcribed region of CXCL12, GREB1, PGR and TFF1 upon SUPT6H knockdown

Fig. 13. Immunohistochemical analysis of human breast tissue and breast cancer sections suggests an inverse correlation between SUPT6H protein and H2Bub1 levels and malignancy

Fig. 14. Immunohistochemical analysis of human breast tissue sections based on hormone receptor status

Fig. 15. SUPT6H regulates ERa-dependent luminal epithelial phenotype.

Fig. 16. SUPT6H knockdown impairs hMSC differentiation to the adipocyte and osteoblast lineages

Fig. 17. SUPT6H regulates $\mathrm{H} 3 \mathrm{~K} 27 \mathrm{me} 3$ on genes during transcription

Fig. 18. SUPT6H regulates $\mathrm{H} 3 \mathrm{~K} 27 \mathrm{me} 3$ on adipocyte-specific genes during transcription

Fig. 19. BRD4 knockdown decreases H2Bub1 levels in MCF10A cells .............................90

Fig. 20. BRD4 and H2Bub1 regulate gene expression in a similar fashion .....................92

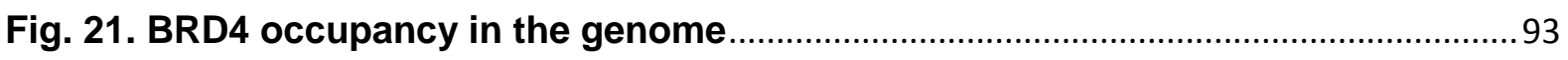


Fig. 22. BRD4 and H2Bub1 occupancy correlate with the expression of the gene ........95

Fig. 23. Proximal and distal occupancy of BRD4 and H2Bub1 …..................................97

Fig. 24. BRD4 and H2Bub1 regulate mammary stem cell gene signature ......................99

Fig. 25. BRD4 and H2Bub1 suppress mammary stem cell phenotype in MCF10A ......100

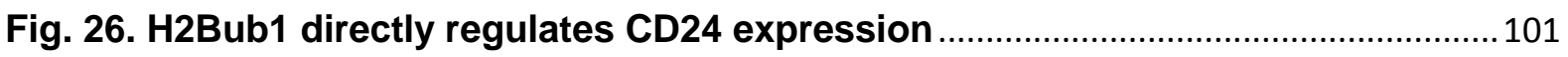

Fig. 27. BRD4 and H2Bub1 regulate the EMT target genes …..................................... 103

Fig. 28. Loss of BRD4 and H2Bub1 induce EMT phenotype in MCF10A cells..............105

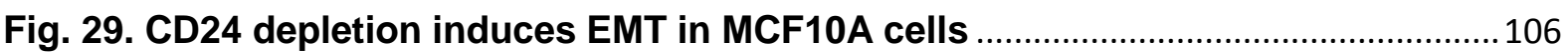

Fig. 30. Confirmation of RNF40 knockout in the mammary glands................................ 106

Fig. 31. Conditional RNF40 KO mice show increased branching of mammary ducts.108

Fig. 32. Model illustrating the role of SUPT6H in transcription

Fig. 33. Possible mechanism of H2Bub1 recruitment by BRD4 associated pathway.. 114

Fig. 34. Graphical representation of the role of BRD4 and H2Bub1 in miRNA regulation. 


\section{Introduction}

\subsection{Chromatin organization and histone modifications}

The human genome is long and contains approximately 6 billion base pairs of DNA which is compacted into fine DNA thread referred to as "Chromatin" (Alberts et al., 2002). Chromatin is composed of the DNA wrapped around a nucleosome containing two of each of the core histones $\mathrm{H} 2 \mathrm{~A}, \mathrm{H} 2 \mathrm{~B}, \mathrm{H} 3$ and $\mathrm{H} 4$. Histones are basic proteins having positive charges which associate and stabilize the negatively charged DNA. They consist of a globular domain and a flexible $\mathrm{N}$-terminal tail (histone tail) projecting out from the nucleosome that undergoes posttranslational modifications (Jenuwein and Allis, 2001). Each of the core histone proteins can be post-translationally modified in various ways including the acetylation, methylation, ubiquitination and sumoylation of the amino terminus of lysine side chains; methylation or citrullination of arginine residues; and the phosphorylation of serine, threonine and tyrosine residues (Jenuwein and Allis, 2001); (Kouzarides, 2007). The specific combination of histone modifications, commonly referred to as the "histone code", is thought to determine the functional outcome probably largely by the recruitment of scaffolding proteins such as bromo- and chromodomain proteins which specifically recognize acetylated and methylated lysine residues, respectively (Jenuwein and Allis, 2001); (James and Frye, 2013). The histone modifications are the most important determinants of the transcriptional state of the genome and it is well recognized that modifications exclusively associated with constitutively silenced regions of the genome constitute "heterochromatin" while other modifications found in actively transcribed regions of the genome form "euchromatin" (Lachner and Jenuwein, 2002). 


\subsection{Epigenetic control}

Epigenetic regulation of gene expression occurs largely through reversible chemical modification of DNA or histone proteins, which do not alter the DNA sequence, but instead control its accessibility and/or ability to be read (Dupont et al., 2009). Other epigenetic changes in chromatin structure can occur through the exchange of variant histones or assembly and disassembly of chromatin structure via histone chaperones, or through ATP-dependent chromatin remodeling, for example, by members of the Swi/Snf family of proteins (Loyola and Almouzni, 2004);(Peterson and Tamkun, 1995). Substantial changes in epigenetic modifications occur to different degrees during various developmental processes such as germ cell development and stem cell differentiation (Hawkins et al., 2010), as well as during pathologic processes such as tumorigenesis (Jones and Baylin, 2007).

\subsection{Epithelial to Mesenchymal Transition}

Metastatic disease accounts for more than $90 \%$ of deaths in patients with solid tumors (Zajicek, 1996). Our understanding of metastasis has been greatly improved by the recognition that cancer cells can acquire the ability to accomplish several steps of the metastatic process at once through the engagement of a cellular program, Epithelial-Mesenchymal Transition (EMT). EMT plays an important role in controlling critical morphogenetic steps during normal embryonic developmental processes such as gastrulation and neural crest migration. In the context of cancer, EMT has been linked to the acquisition of cancer cell motility and invasiveness. During both normal development and tumor progression, EMT is orchestrated by a set of pleiotropically acting transcription factors (TFs), such as Twist, Snail, Slug, Zeb1/2 that together form an intricate transcriptional circuitry (Scheel and Weinberg, 2012). Through the action of EMT-TFs, which mainly act as transcriptional 
repressors, cells lose epithelial traits, such as expression of E-cadherin and ZO-1, leading to the dissolution of adherens and tight junctions (Fig. 1). Repression of epithelial markers is paralleled by upregulation of mesenchymal adhesion molecules, such as $\mathrm{N}$-cadherin and fibronectin. Together, these changes enable epithelial cells to switch from an apical-basal polarity and restricted lateral, collective movement confined by the basement membrane to a front-to-back polarity and ability to freely migrate and invade as single cells. Thus, in embryonic development, EMT is critical for mesoderm formation during gastrulation and overall, EMT affects cellular distribution throughout the embryo during processes such as neural crest migration. In the context of cancer, EMT enables cancer cells to succeed in the early steps of the metastatic process, including local tissue invasion, entry into blood and/or lymphatic vessels, survival during transit and exit from the circulation.

Activation of an EMT program is coupled with the ability of cancer cells to initiate experimental tumors in mice with high efficiency (Bos et al., 2010), although the exact molecular mechanisms linking EMT and tumor-initiating capacity of cancer cells remain to be determined. Given the similarity of experimental tumor initiation and establishment of macroscopic metastases, it is quite likely that EMT is involved in both the early and late steps of the metastatic cascade. In contrast, recent research points to the necessity of the reversal of EMT via a Mesenchymal-Epithelial Transition (MET) at the metastatic site to enable the outgrowth of disseminated tumor cells (DTC) into macroscopic metastases (Peinado et al., 2011). These experimental studies are supported by the clinico-pathological observation that most metastases arising from carcinomas display an epithelial phenotype. However, these seemingly opposing observations may be reconciled by comprehending EMT as a highly dynamic and reversible process. In this scenario, the most aggressive tumor 
cells would be predicted to be those which display a high degree of cellular plasticity. This, in turn, renders inhibition of such plasticity as an attractive approach for therapeutic intervention aimed at inhibiting cell-state transitions, rather than targeting mutated or otherwise genetically altered gene products. However, the precise molecular links between EMT programs and cellular plasticity are only now beginning to be unraveled.

Given the dynamic and drastic transcriptional changes that occur during EMT and MET, broad sweeping, reversible changes in epigenetic modifications which affect chromatin state represent a central and crucial regulatory component of the metastatic process. Changes in gene expression do not depend solely upon the availability of appropriate transcription factors, but also upon the degree of "openness" or "closedness" of the chromatin since both the binding of a TF to DNA as well as its ability to recruit additional transcriptional co-regulatory proteins depends upon changes in histone modifications at the target gene. Emerging data have shown that EMT also involves epigenetic reprogramming with widespread alterations to chromatin modifications at both the DNA and protein level. For example, EMT-TFs, such as Twist, Snail, Slug, ZEB1 and ZEB2 recruit various histone modifying complexes to chromatin, thereby mediating epigenetic silencing of genes. In this section, the interplay between EMT-TFs, transcriptional regulation of EMT markers and chromatin modifiers focusing primarily on histone modifications largely due to their amenability to therapeutic intervention is described.

\subsubsection{Epigenetics and EMT}

Several signaling networks including hypoxia, TGF $\beta$, Wnt and $\mathrm{NOTCH}$ signaling all activate EMT by wide-spread transcriptional changes via the activation of specific transcription factors which elicit their effects on gene transcription and the epigenetic 


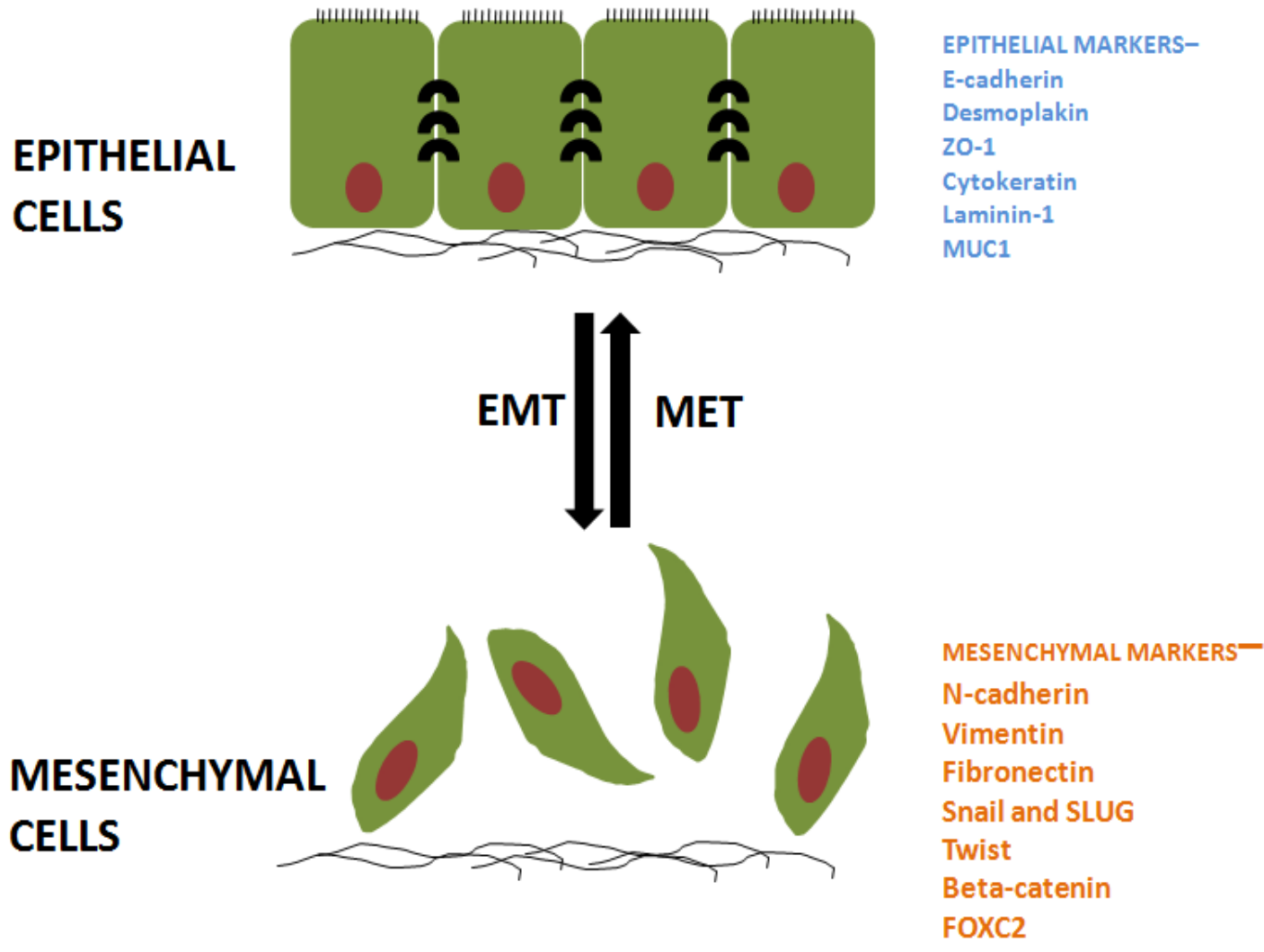

Fig. 1. The process of EMT and its reversible MET. Epithelial cells having a particular set of markers undergo biochemical changes and acquire different set of markers for a mesenchymal phenotype.

landscape by recruiting epigenetic regulatory proteins to specific genes, such as those associated with an epithelial or mesenchymal phenotype. Therefore, a better understanding of the functional interaction of epigenetic modifiers with EMT-TFs and their specificity in the EMT and MET processes may lead to the identification of new therapeutic targets for preventing metastasis.

\subsubsection{Epigenetic Writers}

Epigenetic modifiers can largely be classified into the categories of "epigenetic writers", "epigenetic readers" and "epigenetic erasers". The proteins which catalyze a specific histone modification are referred to as "epigenetic writers". Examples of epigenetic writers include both DNA and histone/lysine methyltransferases 
(HMT/KMT), histone/lysine acetyltransferases (HAT/KAT), arginine methyltransferases (PRMT), ubiquitin ligases, etc. As mentioned earlier, the EMT transcriptional program is controlled both by DNA methylation (Cedar and Bergman, 2009) and post-translational histone modifications (Campos and Reinberg, 2009).

In mammalian cells, DNA is methylated at the cytosine residues of CpG dinucleotides and is commonly associated with gene repression and heterochromatin formation (McCabe et al., 2009). In cancer cells, the genome is globally hypomethylated whereas CpG islands are frequently hypermethylated, resulting in reduced expression of tumor suppressor genes (Baylin et al., 2001). For example, the $\mathrm{CDH} 1$ gene encoding E-cadherin is frequently hypermethylated in breast cancer cell lines exhibiting an EMT-like phenotype (Lombaerts et al., 2006) and is was also shown to be methylated along with several other genes silenced in basal-like breast cancers (Dumont et al., 2008). In addition, to methylation of cytosine, subsequent hydroxylation of $5 \mathrm{mC}$ to 5 -hydroxymethyl-cytosine $(5 \mathrm{hmC})$ by the Ten-Eleven Translocation (TET) family of methylcytosine dioxygenases plays a tumor suppressor function in many types of cancers (Hsu et al., 2012); (Huang et al., 2013); (Yang et al., 2013). Notably, TET1 is frequently down-regulated in breast and prostate cancer in cell lines and xenograft models and its downregulation is associated with overall poorer patient survival (Hsu et al., 2012). This effect appears to be at least partially due to a TET1-dependent demethylation and activation of the Tissue Inhibitor of Metalloproteinase (TIMP)-2 and 3 genes, which are established suppressors of the EMT phenotype. Thus, alterations in both $5 \mathrm{mC}$ and $5 \mathrm{hmC}$ caused by mutation or repression of the TET proteins may play a role in promoting EMT in solid malignancies. A role for the TET proteins in metastasis is also supported by a recent study, demonstrating that the micro-RNA miR-22 exerts its prometastatic 
effects by directly targeting TET proteins (Song et al., 2013). In this way, downregulation of the TET proteins prevents demethylation of the miR-200 gene, which targets the mRNAs for established regulators of the EMT program such as the EMT-TF Zeb1, TGFß1 and the polycomb protein BMI1, thereby potently antagonizing activation of an EMT program and metastasis.

Histone Acetyltransferases (HATs) - An important and well-studied modification responsible for making chromatin accessible to transcription factors is histone acetylation (Grunstein, 1997). Histone acetyltransferases such as GCN5, P/CAF and p300/CBP transfer acetyl groups to the amino group of lysine side chains of histones, thereby altering the charge of the histone, relaxing the chromatin and making it more accessible to transcription factors (Imhof et al., 1997). One important HAT, p300, affects the regulation of Snail and ZEB1 in colon cancer, thereby contributing to EMT and tumor progression (Peña et al., 2006). A different study reported that the absence of p300 promotes EMT in HCT116 colorectal cancer cells (Krubasik et al., 2006). Other HATs such as the human homolog of males absent on the first (hMOF/KAT8) as well as the Steroid Receptor Coactivators-1 and -3 (SRC1/NCOA1 and SRC3/NCOA3) have been shown to play tumor and metastasis suppressor and activator roles, respectively (Jafarnejad and Li, 2012); (Qin et al., 2009); (Agoulnik et al., 2005); (Zhou et al., 2005); (Lydon and O’Malley, 2011). However, whether these or other HATs play specific roles in EMT remains largely unknown.

Histone Methyltransferases (HMTs) - Methyltransferases transfer methyl groups to the lysine or arginine residues of histones. They are classified into lysine (KMT) or arginine methyltransferases (PRMT) depending on the substrate residue for methylation. SET ( $\underline{\text { Su}(v a r) ~ 3-9, ~ E n h a n c e r ~ o f ~ Z e s t e ~ a n d ~ T r i t h o r a x) ~ d o m a i n ~ c o n t a i n i n g ~}$ 
enzymes such as G9a, SUV39H1/H2, EZH1/2 and others, transfer one to three methyl groups to lysine residues on histones (Miller et al., 2001); (Wang and Shang, 2013). Expressed genes typically display "active" methylation marks such as H3K4me3, H3K36me3 and H3K79me3, while transcriptionally silenced genes generally exhibit "repressive" marks such as H3K27me3, H3K9me2 and H3K9me3. Upon hypoxia, mesenchymal genes are marked with H3K4me3 by WDR5, part of MLL and SET1 HMT complex (Wu et al., 2011a). The Polycomb Repressor Complex-2 (PRC2), which contains the methyltransferase Enhancer of Zeste Homolog-2 (EZH2) in complex with Suppressor of Zeste-12 (SUZ12) and Embryonic Ectoderm Development (EED) (Ringrose and Paro, 2004), plays a key role in transcriptional silencing by mediating H3K27me3 (Orlando, 2003). The role of PRC2 in tumorigenesis and EMT has been well characterized and its interplay with EMTTFs is described in more detail below.

Histone Ubiquitin Ligases - Ubiquitination involves the attachment of one or more 76 amino acid ubiquitin moieties to the side change of a lysine in a process involving the sequential function of three enzymes: E1 ubiquitin-activating, E2 ubiquitinconjugating and E3 ubiquitin-ligase enzymes (Hershko and Ciechanover, 1998). While polyubiquitination via lysine 48 of ubiquitin frequently targets proteins for degradation via the $26 \mathrm{~S}$ proteasome, monoubiquitination does not usually target proteins for degradation, but rather functions like other post-translational modifications to serve as a mark for recognition by other proteins or directly alter protein structure or function. In the case of chromatin, both histones $\mathrm{H} 2 \mathrm{~A}$ and $\mathrm{H} 2 \mathrm{~B}$ can be monoubiquitinated in mammals at Lys-119 (H2Aub1) or Lys-120 (H2Bub1), respectively. H2Bub1 is generally associated with euchromatin and transcriptional elongation (Xiao et al., 2005); (Shukla et al., 2006); Minsky et al. 2008) whereas 
$\mathrm{H} 2 \mathrm{Aub1}$ is localized to regions of heterochromatin and prevents transcriptional elongation (Wang et al. 2004a; (Cao et al., 2005). H2B is monoubiquitinated by the obligate RNF20/40 heterodimer in a complex with the ubiquitin conjugating enzyme UBE2A (human homolog of yeast Rad6A) (Zhu et al., 2005a). While decreased H2Bub1 levels are associated with increased invasiveness and tumor progression, its role in controlling EMT has not been described (Johnsen, 2012a); Shema et al. 2008; (Prenzel et al., 2011). In contrast, components of the Polycomb Repressor Complex-1 (PRC1), which ubiquitinates H2A have been shown to promote EMT by upregulating Snail via modulation of PI3K/Akt/GSK-3 $\beta$ signaling (Song et al., 2009) as well as targeting other important EMT transcription factors such as Twist1 and ZEB1 (Yang et al., 2010); Wellner et al. 2009).

\subsubsection{Epigenetic Readers}

Once the chromatin has been marked with specific post-translational histone modifications, the regulatory output in most cases is achieved by the recognition of those marks by epigenetic readers. These chromatin regulators possess specialized domains that recognize and bind to various histone modifications and control DNAassociated functions by recruiting additional regulatory proteins and/or by directly affecting chromatin structure (de la Cruz et al., 2005).

Bromodomains - Bromodomain-containing proteins recognize acetylated lysine residues (Haynes et al., 1992); (Jeanmougin et al., 1997). One particularly noteworthy subclass of bromodomain proteins is the BET (Bromodomain and Extra Terminal) family of proteins which contain two tandem bromodomains at the $\mathrm{N}$ terminal and an Extraterminal domain (ET) at the C-terminus (Florence and Faller, 2001). The family contains BRD2, 3, and 4 as well as the testis-specific BRDT protein and is implicated in transcription regulation by binding to chromatin by virtue 
of the bromodomains (Wu and Chiang, 2007a). In addition to its established role in promoting leukemiogenesis by MLL translocation products (Zuber et al., 2011a) and its fusion with NUT in NUT midline carcinoma (French et al., 2003); (French et al., 2004); (French et al., 2007), BRD4 was shown to suppress an EMT phenotype in mammary epithelial cells (Alsarraj et al., 2011a). However, additional studies are necessary to further characterize the function and molecular mechanisms of BRD4 and other BET domain proteins in EMT during tumor progression and metastasis.

Recognition of methylated lysine residues - Analogous to the recognition of acetylated lysine residues by bromodomains, a number of different domains have been identified which recognize methylated lysine residues including Chromatin organization modifier (chromo-), TUDOR, Plant 브omeodomain (PHD) and Malignant Brain Iumor (MBT) domains (Kim et al., 2006). SFMBT1, a MBT domain containing protein and part of LSD1 complex, gets recruited to epithelial genes via SNAl1 mediated interaction and promotes gene repression by demethylation of $\mathrm{H} 3 \mathrm{~K} 4 \mathrm{me} 2$ (Tang et al., 2013). Included in the chromodomain family of proteins are three subfamilies which include the heterochromatin protein (HP1)/chromobox (CBX) proteins, the chromodomain helicase DNA binding domain (CHD) subfamily and the chromo barrel domain family (Yap and Zhou, 2011). CBX proteins are components of PRC1 complex which recognizes $\mathrm{H} 3 \mathrm{~K} 27 \mathrm{me} 3$ to promote $\mathrm{H} 2 \mathrm{Aub} 1$ and transcriptional repression at PRC2 targets (Levine et al., 2002); (Francis et al., 2004). CBX4

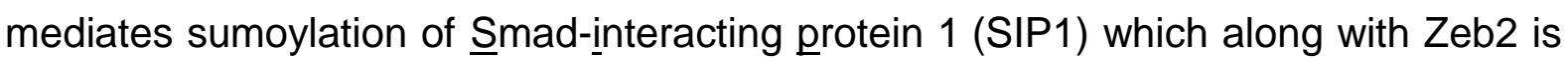
involved in CDH1 repression and EMT (Long et al., 2005); (Vandewalle et al., 2005). Another member, MPP8 (M-phase phosphoprotein 8) recognizes H3K9 methylation on the chromatin and interacts with HMTases GLP and ESET as well as DNA 
methyltransferase, DNMT3A. MPP8 in turn functions to repress $\mathrm{CDH} 1$ expression thereby promoting EMT (Kokura et al., 2010).

Chromatin remodeling proteins and histone chaperones - The regulation of chromatin organization and structure requires both the ATP-dependent activity of chromatin remodeling proteins as well as the ATP-independent functions of histone chaperones (Sif, 2004);(Avvakumov et al., 2011). One component of the Swi/Snf family of ATP-dependent chromatin remodeling proteins BRG1 was found to be mutated in various human tumor cell lines (Wong et al., 2000) and appears to function with beta catenin at TCF target gene promoters to facilitate $\mathrm{Wnt} / \mathrm{\beta}$-cateninregulated gene transcription in colon carcinoma cells (Barker et al., 2001). Importantly, BRG1 also interacts directly with the EMT-TF ZEB1 to repress $C D H 1$ expression and promote EMT (Sánchez-Tilló et al., 2010). In contrast, Metastasisassociated gene 3 (MTA3), part of the ATP-dependent NuRD/Mi-2/CHD remodeling complex was shown to suppress EMT by directly repressing SNAl1 expression (Fujita et al., 2003). Although the activity of histone chaperones has not yet been linked to EMT, our recent data identified decreased expression of the human Suppressor of Ty Homologue-6 (SUPT6H) during breast cancer progression which was associated with decreased H2Bub1 levels, a loss of estrogen responsiveness and a shift from a luminal epithelial to myoepithelial phenotype (Bedi et al. 2013, In Press Oncogene). Another histone chaperone complex referred to as Facilitates Chromatin Transcription (FACT) has been implicated in tumorigenesis (Koman et al., 2012) and DNA repair (Kari et al., 2011a). Interestingly, an analysis of gene expression data from the Cancer Cell Line Encyclopedia (Barretina et al., 2012)

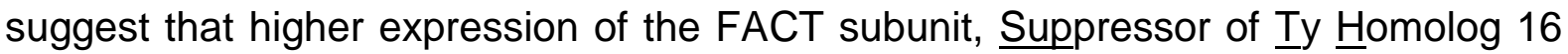
(SUPT16H) is more closely correlated with expression of the epithelial markers 
$\mathrm{CDH} 1, \mathrm{CRB} 3, \mathrm{PKP3}$ and $\mathrm{CDH} 3$, and inversely correlated with the expression of the mesenchymal markers AXL, FN1, SNAI2, VIM, CDH2, TWIST1 and ZEB1 (Fig. 2). Thus, whether and how FACT activity is correlated with an EMT phenotype may be of particular relevance for the application of molecules targeting FACT activity. Future studies will be necessary to determine whether and how SUPT6H, SSRP1, SUPT16H and other histone chaperones promote an EMT phenotype.

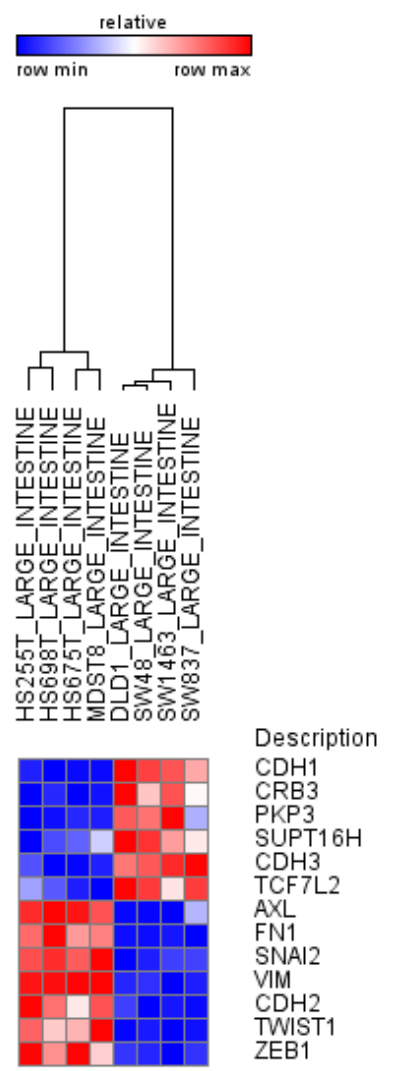

Fig. 2. SUPT16H, FACT subunit correlates with the epithelial phenotype in human intestinal cell lines. Analysis using data from Cancel Cell Line Encyclopedia indicates increased expression of epithelial markers for SUPT16H and decreased expression of mesenchymal markers in intestinal cell lines.

\subsubsection{Epigenetic Erasers}

In contrast to the notion of epigenetic inheritance, most epigenetic modifications, including extremely stable modifications such as DNA methylation, are highly dynamic and can be added or removed from genes within a matter of minutes, 
frequently in a cyclic fashion (Métivier et al., 2008); (Kangaspeska et al., 2008); (Métivier et al., 2003). After the initial activation or repression of a gene has been achieved, cellular and transcriptional plasticity is maintained by the reversibility of the epigenetic status of the target genes. In order to achieve this, most histone modifications also have specific enzymes which catalyze their removal. This class of proteins is broadly referred to as "epigenetic erasers" and exerts an equally important function as writers. If the signal is not stopped timely, the results can lead to defects in transcription and DNA repair ultimately promoting tumorigenesis of tumor progression (Arrowsmith et al., 2012).

Histone Deacetylases (HDACs) - The acetyl groups added by HATs are removed by HDACs in a highly regulated fashion and generally leads to chromatin compaction and transcriptional repression. Notably, HDAC1 was found to be important for TGF $\beta 1$ induced EMT (Lei et al., 2010) and its inhibition suppressed TGF $\beta 1$ induced EMT (Yoshikawa et al., 2007). HDAC3 also interacts with WDR5, a core component of the histone methyltransferase complex responsible for H3K4 methylation and induced hypoxia-mediated EMT by regulating acetylation and methylation patterns on EMT genes (Wu et al., 2011b). Furthermore, the $\mathrm{NAD}^{+}$-dependent histone deacetylase SIRT1 was shown to cooperate with ZEB1 to silence $C D H 1$ expression by deacetylating its promoter (Byles et al., 2012).

Histone demethylases - Finally, histone demethylases revert the effect of methylases by removing the repressive marks on histones. The first histone demethylase to be identified that removes mono- and di- methyl groups on H3K4 was, Lysine-Specific Demethylase-1 (LSD1) or KDM1A (Shi et al., 2004). During EMT, SNAl1 recruits LSD1 to epithelial gene promoters for repression by removal of dimethylation of H3K4me2 on their promoters (Lin et al., 2010a); (Ferrari-Amorotti et 
al., 2013); (Amente et al., 2013). Two other demethylases belonging to the Jumonjidomain family, KDM6B (JMJD3) and JMJD2B which remove H3K27me3 and H3K9me3, respectively, were both recently shown to promote EMT as well (Ramadoss et al. 2012a; (Zhao et al., 2013).

Histone deubiquitinases (DUBs) - As with essentially all other post-translational modifications, the ubiquitin moiety from histone can also be removed in order to reverse the effects of ubiquitination. One example is Ubiquitin-specific-protease-22 (USP22) which deubiquitylates histone H2B (Zhang et al., 2008a) and was found to regulate BMl1 mediated INK4a/ARF and Akt signaling (Liu et al., 2012). Consistently, USP22 is upregulated in tumors with a stem cell-like phenotype exhibiting a poor patient outcome (Glinsky et al., 2005); Zhang et al. 2008; Liu et al. 2011). While many arrows point in the direction that the positive and negative regulation of $\mathrm{H} 2 \mathrm{Bub} 1$ could be associated with a tumor stem cell-like phenotype and EMT, further work will be needed to address this. The ubiquitination of H2A was reported to be reversed by a number of different DUBs including USP3 (Nicassio et al., 2007). Notably, USP3 depletion induces scattering of A549 epithelial lung cancer cells, possibly reflecting a more mesenchymal cellular phenotype (Buus et al., 2009). However, how and whether H2A deubiquitination is involved in controlling EMT must be more clearly addressed.

\subsubsection{Epigenetic Regulation of EMT inducing Transcription factors}

The cellular plasticity which allows the interconversion between epithelial and mesenchymal phenotypes via EMT and MET requires a complicated network of interactions between EMT-TFs, ubiquitous TFs and the epigenetic regulators described above. In this case, both the expression and the activity of EMT-TFs are controlled at an epigenetic level. The connection between loss of E-cadherin and 
tumor progression has been well established (Tepass et al., 2000), and studies have highlighted the epigenetic regulation of the $C D H 1$ gene encoding E-cadherin to be instrumental for cancer cell metastasis (Birchmeier and Behrens, 1994). CDH1 expression is regulated by EMT-TFs including the Snail transcription factor family members Snail (SNAI1) and Slug (SNAI2) (Batlle et al., 2000). Studies have shown that Snail recruits several chromatin modifying enzymes, such as LSD1, G9a, Suv39H1, HDAC1/2 and PRC2, to the $C D H 1$ promoter for transcriptional repression (Dong et al., 2013); (Dong et al., 2012); (Herranz et al., 2008); (Lin et al., 2010b). Fig. 3 lists the described interactions of EMT-inducing factors with various epigenetic factors to transcriptionally repress epithelial genes during EMT. While the epigenetic regulation of EMT markers has been studied for a number of years, only recently has the focus been shifted to also investigate the epigenetic control of EMT-TF expression.

SOX4 - an important upstream regulator of the EMT program - SOX4 is a member of the Sox (SRY-related HMG-box) family of transcription factors and is frequently upregulated in various cancer types (Liu et al., 2006). A recent study demonstrated that SOX4 acts early in the induction of the EMT pathway (Tiwari et al., 2013). Upon TGF $\beta 1$ induction, SOX4 expression is increased, thereby transcriptionally activating EZH2 expression, which in turn increases H3K27me3 at specific genes in order to promote EMT. In concordance, depletion of either the transcription factor SOX4 or its epigenetic regulatory partner EZH2 similarly prevented TGF $\beta$-induced EMT in the murine mammary epithelial cell line NMuMG. In contrast, SOX4 overexpression induced EMT via modulation of Ezh2-mediated H3K27me3 marks on important EMT genes. Together these results strongly implicate SOX4 as a critical upstream 


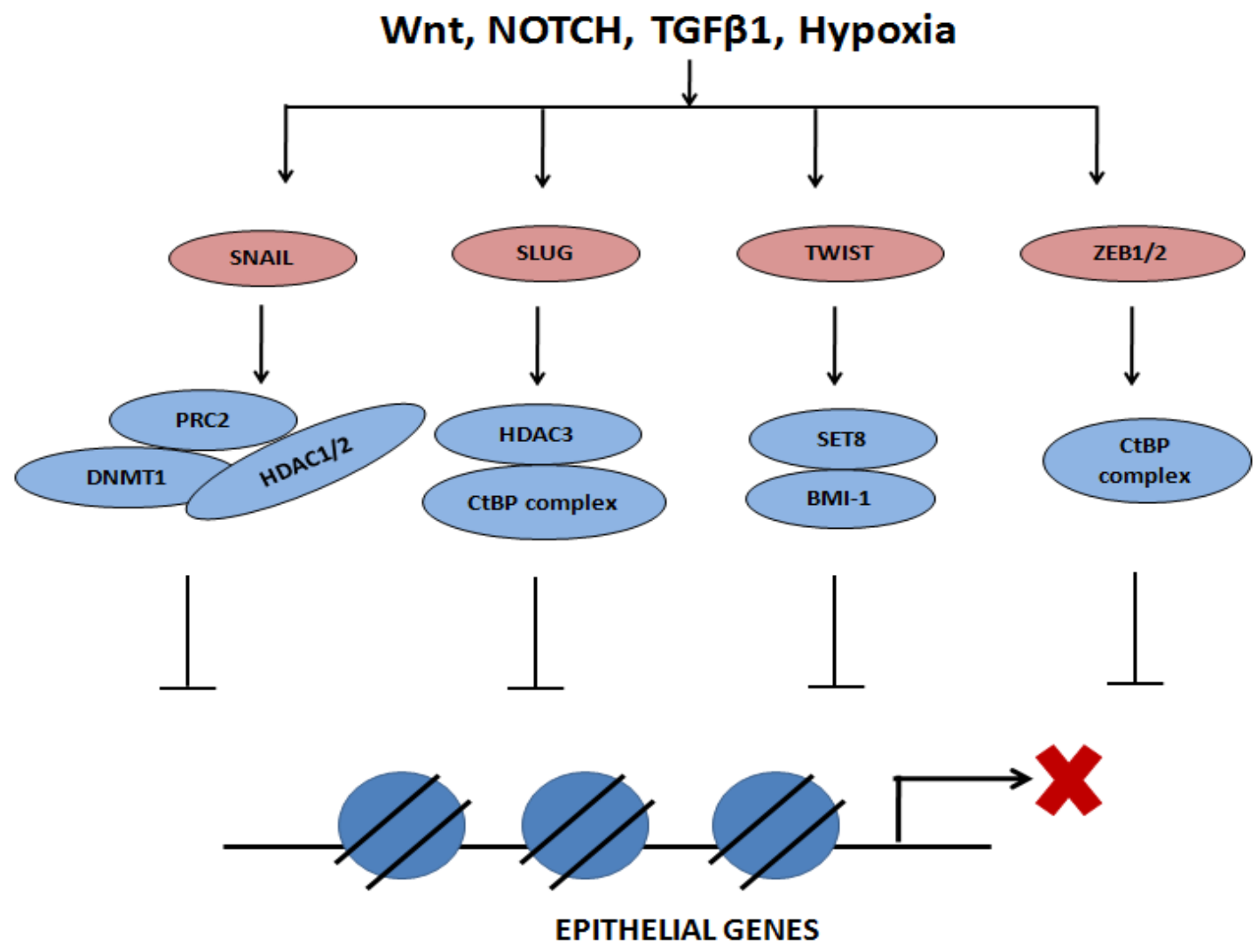

Fig. 3. EMT-TFs interact with epigenetic regulators to repress epithelial genes. EMT inducing factors activate the EMT-TFs which in turn interact with epigenetic regulators to repress the expression of epithelial genes.

regulator of the EMT modulators which carries out its function via epigenetic mechanisms involving EZH2.

SUV39H1 (Suppressor of Variegation $\underline{3-9} \underline{\text { Homolog }} 1$ ), is a key methyltransferase responsible for $\mathrm{H} 3 \mathrm{~K} 9 \mathrm{me} 3$. $\mathrm{H} 3 \mathrm{~K} 9 \mathrm{me}$, like $\mathrm{H} 3 \mathrm{~K} 27 \mathrm{me}$, is a histone modification associated with gene repression. It was shown recently that upon TGF $\beta 1$ induction in MCF10A, there was an upregulation of SUV39H1 along with Snail. These factors have been shown to interact with each other and establish a repressed state of the CDH1 promoter by increasing the levels of H3K9me3 (Dong et al., 2013). 
ZEB1 and ZEB2 are key factors regulating CDH1 expression and their connection with EMT and metastasis of cancer cells has been well established (Spaderna et al., 2008). In a recent study it has been shown that the ZEB1 promoter exists in a poised state containing both markers of activation (H3K4me3) and repression (H3K27me3) (Chaffer et al., 2013). In epithelial cells, ZEB1 is not expressed due to the bivalent marks on its promoter, but upon EMT induction by factors such as TGF $\beta 1$, there is removal of H3K27me3 marks on the ZEB1 promoter leading to its expression. Similarly, removal of H3K27me3 by KDM6B was also shown to be essential for the induction of SNAl1 expression during TGF $\beta 1$-induced EMT (Ramadoss et al., 2012).

Apart from histone methylation, histone acetylation on the genes of the EMT transcription factors is also being investigated. In this regard, it was previously shown that histone deacetylases (HDACs) modulated the chromatin state upon stimulus of extracellular signals like hypoxia (Wu et al., 2011b). Upon hypoxia, a well-described inducer of cancer cell aggressiveness and EMT, HDAC3 was recruited to epithelial genes like $\mathrm{CDH} 1$ leading to decreased $\mathrm{H} 3 \mathrm{~K} 4 \mathrm{ac}$, in turn increased $\mathrm{H} 3 \mathrm{~K} 4 \mathrm{me} 2$ and $\mathrm{H} 3 \mathrm{~K} 27 \mathrm{me} 3$ on their promoters. This led to a repression of epithelial genes. On the other hand, mesenchymal genes like Vimentin had decreased H3K4ac, increased H3K4me2 but decreased H3K27me3. HDAC3 interacted with WDR5 which led to methylation of H3K4 in hypoxic cells.

It was also shown in lung cancer that ZEB1 downregulated its target genes like EpCam (epithelial cell adhesion molecule) by decreasing H3K27ac on these genes (Roche et al., 2013). These findings are critical in understanding that the epigenetic regulation occurs quite upstream of these markers or transcription factors, which may lead to the evaluation of these upstream EMT regulators as potential targets in anti-metastatic therapy. Fig. 4 demonstrates the cascade of epigenetic events that 
control the transcriptional regulation of EMT transcription factors in response to EMT stimuli. The EMT TFs further execute their task of epigenetically regulating the transcription of epithelial and mesenchymal genes.

As EMT has been well studied in particular for the initial transformation and tumor progression, the reversal may be equally important for malignant cells. Depicted in Fig. 1, the Mesenchymal to Epithelial transition (MET) certainly must be more carefully addressed and characterized at the molecular level as it is considered to allow systemically dispersed tumor cells (i.e. DTCs) to regain epithelial characteristics, starting to grow and giving rise to clinically overt metastases ( $\mathrm{K}$ and U, 2003). Simplified, it is thought that after the cells have migrated to distant sites, they no longer receive the EMT-inducing signals experienced in the primary tumor environment, thus allowing them to easily revert back to the epithelial phenotype through MET. These transitions are almost certainly based largely on epigenetic plasticity.

\subsubsection{Cancer stem cell hypothesis}

Another important aspect of EMT is the acquisition of stem cell-like traits to form so called cancer stem cells (CSC) or tumor-initiating cells (TICs). CSCs are recognized by their ability to efficiently give rise to tumors when injected into immunocompromised mouse models due to their self-renewal properties (Reya et al., 2001). Since the first reports in 2008, the molecular connections between EMT and stem cell traits have constantly emerged over the past few years. It is thought that while undergoing metastasis, cancer cells stem cell characteristics which fuel tumor growth at sites of colonization. In doing so, the whole set of EMT master regulators may initiate the epigenetic switch that modulates the gene expression of stem cell markers. After several years, it is now clear that cancer stem cells can be 
H3K27me3

H3К9me2

нзк9me3

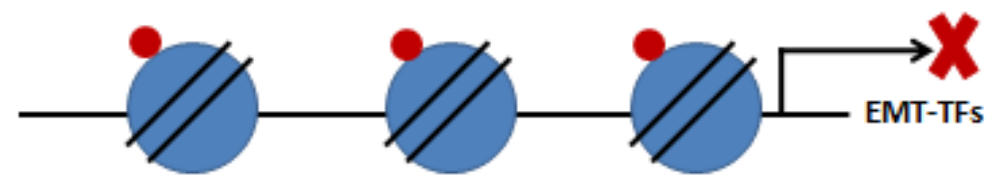

H3K4me2

H3K4me3

HзК36me3

H3K79me3

H3К9ас
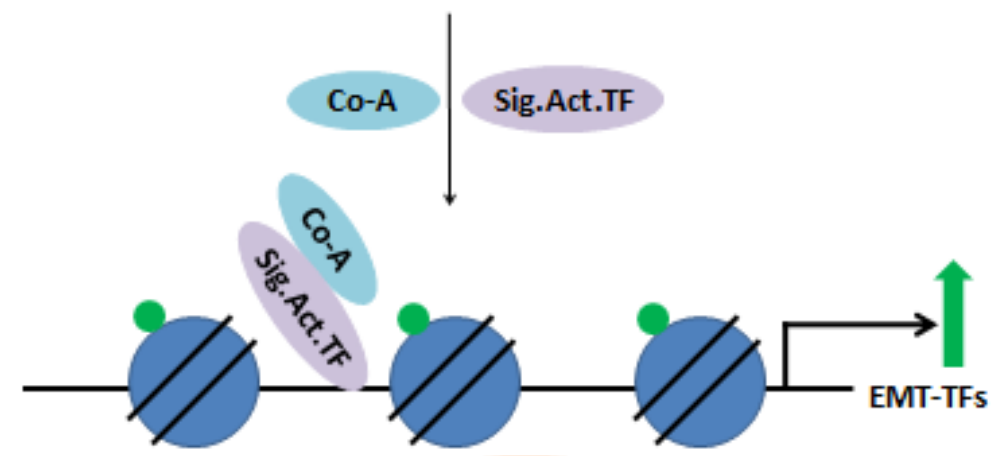

Co-R Co-repressor

Co-A Co-activator

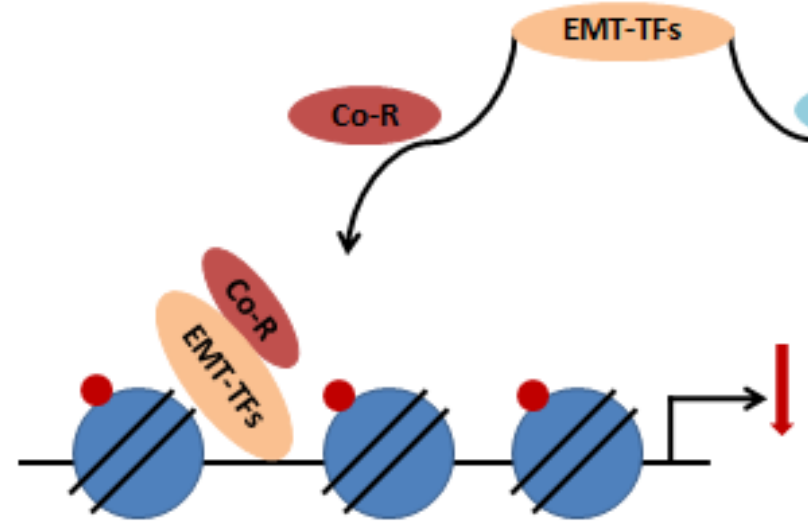

EPITHELIAL MARKERS

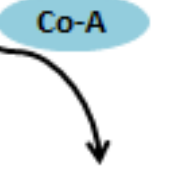

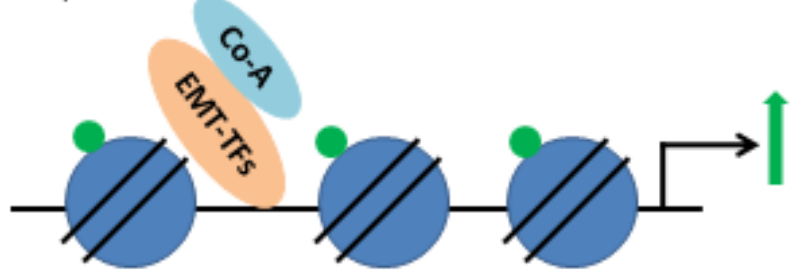

MESENCHYMAL MARKERS

Fig. 4. Signaling activators induce transcription of EMT-TFs which in turn regulate histone modifications on target genes. Upon inducing signal, activating histone modifications on the genes of EMT-TFs promote transcription. EMT-TFs then interact with epigenetic regulators to mark the target genes for activation or repression.

identified based on their cell surface marker profile, e.g. CD44 ${ }^{\text {hi }} / \mathrm{CD}_{2} 4^{\text {lo }}$ or $\mathrm{ALDH}^{+}$in breast cancer (Al-Hajj et al., 2003a). It was shown that ZEB1 promotes the switch in these markers in a given cell population, rendering these cells more stem cell-like (Chaffer et al., 2013). Additionally, ZEB1 can also negatively regulate miR200b expression which functions to suppress the stemness of cells (Park et al., 2008). The 
miR200b family further suppresses polycomb protein BMl1 expression, which is known to exert stemness regulation (Shimono et al., 2009).

Based on genome-wide occupancy studies for various histone modifications, it was observed that the two cancer cell populations, $\mathrm{CD}_{4} 4^{+}$(mesenchymal-like) and $\mathrm{CD}^{+} 4^{+}$(epithelial-like) had different methylation patterns clearly demonstrating epigenetic regulation of epithelial and mesenchymal gene expression (Maruyama et al., 2011). An expression-methylation correlation was also shown, implying that the methylation pattern could be an important determinant of gene expression in cancer cells, especially in terms of EMT and stemness properties (Kamalakaran et al., 2011).

\subsection{Estrogen signaling and Estrogen Receptor}

The steroid hormone estrogen plays an important role in mammalian reproduction. Estrogens regulate several physiological processes, including normal cell growth, development, and maintenance of reproductive tract and bone density as well as regulation of central nervous and skeletal systems (Couse, 1999); (Pettersson and Gustafsson, 2001).

The biological action of estrogen is mainly mediated by the Estrogen Receptor (ER) to which estrogens bind. There are two isoforms of ER, namely ERa and ERß, each encoded by unique genes, ESR1 and ESR2 respectively (Green et al., 1986); (Kuiper et al., 1996). In order to study the role of two receptor isoforms in estrogen signaling, knockout mice for ER were generated to examine the effects on reproductive system. The aERKO female mice having loss of ERa were infertile and showed phenotype such as estrogen-insensitive uteri, little sexual behavior and underdeveloped mammary glands. The aERKO male mice were also infertile. On the 
other hand, the BERKO females were subfertile, producing fewer litters and showed normal mammary gland structure. The BERKO male fertility was unaffected (Couse et al., 2000); (Hewitt et al., 2000) (Emmen and Korach, 2009). Since the effects of aERKO mice were more pronounced, the ERa is widely studied for the estrogen signaling.

\subsubsection{Estrogen Receptor Alpha (ERa)}

The estrogen receptor $\alpha(E R \alpha)$ is one of the key transcriptional regulators of proliferation and differentiation in the mammary epithelium(Deroo and Korach, 2006). Approximately two-thirds of human breast cancers express ERa. ERa expression is associated with a more differentiated luminal tumor phenotype and overall better patient survival compared to ERa negative tumors (Ali and Coombes, 2000);(Khan et al., 1998). Thus, ERa expression is an important prognostic marker and is predictive for tumor response to anti-estrogen treatment. Despite an initial positive response, roughly one-third of ERa-positive tumors becomes refractory to anti-hormone therapy and develops estrogen-independence. Therefore, an increased understanding of the molecular mechanisms controlling ERa-mediated transcriptional regulation may help to uncover new molecular targets which may be utilized to more effectively treat and eradicate ERa-positive tumors (Theodorou et al., 2013).

Recent studies have demonstrated that one of the essential transcriptional regulatory steps controlled by ERa is transcriptional elongation (Kininis et al., 2009). ERa interacts with the Positive Transcription Elongation Factor-b (P-TEFb) complex (Wittmann et al., 2005) which promotes transcriptional elongation in part by phosphorylating Ser2 (P-Ser2) within the carboxy-terminal domain (CTD) of RNA Polymerase II (RNAPII) (Egloff and Murphy, 2008a). HEXIM1, a negative regulator of 
P-TEFb activity also interacts with ERa (Ogba et al., 2008) and its overexpression leads to a tamoxifen-resistant phenotype in breast cancer cells (Ketchart et al., 2011).

ERa-regulated transcription is a highly dynamic process which is associated with the recruitment of multiple histone modifying enzymes, changes in histone modifications, chromatin remodeling and significant changes in overall chromatin organization (Kouzarides, 2007) Li et al. 2007; (Fullwood et al., 2009) (Métivier et al., 2006). These changes appear to be required for the efficient expression of estrogenregulated genes and may represent new potential therapeutic targets (Johnsen et al., 2006). In previous studies, we uncovered a tumor suppressor function for H2Bub1 (Prenzel et al., 2011) Shema et al. 2008; (Johnsen, 2012b) where its levels decrease during the malignant progression of breast cancer. Interestingly, while H2Bub1 is required for ERa-regulated gene transcription, instead of leading to impaired cell proliferation, a loss of H2Bub1 instead led to the estrogen-independent growth of ERa-positive MCF7 breast cancer cells (Prenzel et al., 2011) possibly implicating a loss of $\mathrm{H} 2 \mathrm{Bub1}$ in the transition from an estrogen-dependent to hormone-independent growth of breast cancer.

\subsubsection{Mechanism of action of ERa}

ER belongs to the nuclear hormone receptor superfamily. There have been several attempts to elucidate the mechanism of action of ER. Earlier studies indicated the "two step mechanism" where ER is a cytoplasmic protein and remains associated with heat shock proteins. Upon ligand binding, ER dissociates from the protein complex and gets localized to the nucleus to regulate transcription of target genes (Jensen and DeSombre, 1973); (Parker, 1995). However, over the years, the model has evolved from the two step mechanism to "one step mechanism". In this classical 
mechanism, estradiol or estrogen diffuses into the cell and binds to nuclear ER resulting in conformation change and dimerization of ER. This complex then binds to the palindromic sequences, Estrogen Response Elements (ERE) of the target genes along with other mediators (White and Parker, 1998); (Tsai and O'Malley, 1994).

The steroid receptors mediate transcriptional activation by two activation functions, $\mathrm{AF} 1$ and AF2, located in $\mathrm{N}$-terminal and hormone binding domain respectively (Lees et al., 1989); (Tora et al., 1989). AF1 has been shown to be phosphorylated by several signaling pathways (Ali et al., 1993); (Bunone et al., 1996). AF2 is induced upon hormone binding and is important for the receptor function (Danielian et al., 1992); (Saatcioglu et al., 1993).

\subsection{Histone H2B monoubiquitination (H2Bub1)}

Ubiquitination is a process of addition of 76 amino acid ubiquitin moiety to the substrate proteins (Hochstrasser, 1996) in a stepwise process catalyzed by three enzymes, mainly ATP-dependent ubiquitin-activating enzyme or E1, a ubiquitinconjugating enzyme or E2, and a ubiquitin ligase or E3 (Glickman and Ciechanover, 2002). E3 enzymes typically consist of RING-finger domain essential for ubiquitin ligase activity. Substrate proteins can be poly- or mono- ubiquitinated in which polyubiquitination marks them for degration by $26 \mathrm{~S}$ proteasome machinery and monoubiquitination is critical for cellular function. Based on the data from the yeast, H2B gets monoubiquitinated at Lysine 123 by the E3 ligase, Bre1 with E2 conjugating enzyme, Rad6 (Wood et al., 2003); (Hwang et al., 2003). In mammals, H2B was found to be ubiquitinated at Lysine 120 by the Bre1 homolog, RNF20/RNF40 complex (Kim et al., 2005); (Zhu et al., 2005b). Importantly, both RNF20 and RNF40 consist of a RING-finger domain but only RING-domain of RNF20 participates in the ubiquitination of H2B in vitro (Kim et al., 2009a). In 
mammalian cells, RNF20/40 complex interacts with hRAD6 (UBE2A), E2 conjugating enzyme as well as hPAF complex and thereby regulates $\mathrm{H} 2 \mathrm{~B}$ monoubiquitination (Kim et al., 2009a).

H2B monoubiquitination is involved in numerous cellular functions, including transcription regulation.

\subsubsection{H2Bub1 and Transcription}

H2Bub1 has been found to play critical role in transcriptional regulation by altering the expression of a subset of genes in mammalian cells (Shema et al., 2008b) Several independent studies have established the positive role of H2Bub1 in transcription. First evidence of $\mathrm{H} 2 \mathrm{Bub1}$ as a transcriptional co-activator was shown by (Kim et al., 2005) in which RNF20 interacted with transcription factor, p53 and was recruited to MDM2 promoter in a p53-dependent manner. Overexpression of both RNF20 and p53 promoted induction of p53 target genes, p21 and MDM2 while depletion decreased the activation. In this way, H2Bub1 has a promoter recruitment mechanism involving direct activator interactions (Kim et al., 2005). H2Bub1 was shown to expedite FACT function thereby enhancing transcription elongation (Pavri et al., 2006). FACT is $\mathrm{H} 2 \mathrm{~A}-\mathrm{H} 2 \mathrm{~B}$ chaperone and plays an important role in nucleosome assembly and resassembly during transcription (Schwabish and Struhl, 2004). In yeast cells, H2Bub1 was shown to interact with FACT subunit, Spt16 and they both regulated nucleosome density and prevented cryptic transcription initiation (Fleming et al., 2008). Its role in transcription elongation has been further strengthened due to its association with the transcribed region of highly expressed genes in mammalian cells (Minsky et al., 2008b). Moreover, it is well established that $\mathrm{H} 2 \mathrm{Bub} 1$ is required for $\mathrm{H} 3$ methylation, both $\mathrm{H} 3 \mathrm{~K} 4$ and $\mathrm{H} 3 \mathrm{~K} 79$ by co-ordinating with COMPASS, complex containing Set1 histone methyltransferase (Lee et al., 2007). 
These marks are considered to be active marks for gene transcription. It was further shown that the addition of huge ubiquitin moiety on H2B led to local disruption of chromatin structure making the site of addition more open and accessible (Fierz et al., 2011a) which facilitates the recruitment of several transcription factors.

Moreover, H2Bub1 was shown to regulate the histone mRNA 3'end processing and critical for correct stem loop-dependent processing of histone genes. CDK9 was also shown to regulate H2Bub1 through CTD-dependent PAF-RNF20/40 complex (Pirngruber et al., 2009a). Thus, CDK9 and H2Bub1 together played an important role in histone mRNA processing.

H2Bub1 has also been shown to participate in cellular processes such as cell cycle checkpoint activation and DNA damage signaling (Chernikova et al. 2012; (Moyal et al., 2011) (Kari et al., 2011a) (Nakamura et al., 2011). Like transcription, DNA repair also requires extensive chromatin reorganization. As H2Bub1 is known to facilitate chromatin remodeling, RNF20/RNF40 mediated H2Bub1 at double strand breaks was found to help in chromatin opening and homologous recombination (Nakamura et al., 2011); Kari et al. 2011). It was also shown that the E3 ubiquitin ligases RNF20 and RNF40 were substrates of the ATM and ATR kinases (Mu et al., 2007) (Stokes et al., 2007).

The role of H2Bub1 has been well recognized in cellular differentiation and estrogen signaling. It was shown that H2Bub1 promotes differentiation of mesenchymal stem cells by resolving the bivalency on differentiation genes and promoting differentiation (Karpiuk et al., 2012a). H2Bub1 levels increased in mesenchymal stem cell differentiation as well as during embryonic stem cell differentiation (Fuchs et al., 2012). 


\subsubsection{H2Bub1 and cancer}

The role of RNF20 as a tumor suppressor was first brought into light by Shema et al. 2008. They showed that RNF20 promoter gets hypermethylated during breast cancer. The depletion of RNF20 led to enhanced cell migration and tumorigenesis as well as decreased p53 levels, an important tumor suppressor. Later it was also shown for RNF40 to regulate estrogen independent cell proliferation and activation of certain cell survival signaling pathways in breast cancer cells (Prenzel et al., 2011). Direct studies on human breast tissues indicated a decrease in H2Bub1 levels in malignant tissues whereas the non-transformed adjacent tissues possessed abundant H2Bub1 (Prenzel et al., 2011).

Recently, RNF20 levels were found to be downregulated in testicular seminomas and in the premalignant lesion in situ carcinoma (Chernikova et al., 2012b). They proposed that upon RNF20 depletion, there is formation of RNA:DNA hybrids referred to as R-loops which are major source of genomic instability. This genomic instability due to loss of RNF20 leads to acquisition of malignant and invasive phenotypes (Chernikova et al., 2012b); (Chernikova and Brown, 2012). Several studies have reported that there is loss of H2Bub1 during carcinogenesis ((Shema et al., 2008a)(Prenzel et al., 2011) (Hahn et al., 2012); (Urasaki et al., 2012). Collectively, these findings suggest that the loss of H2Bub1 correlates with increased cancer progression.

\subsubsection{Histone Deubiquitinases}

Ubiquitination is a reversible process. H2Bub1 is highly dynamic and is maintained by the addition as well as active removal from the chromatin by the class of enzymes called deubiquitinases (DUBs). 
In yeast, Spt-Ada-Gcn5-Acetylating complex (SAGA), a novel transcriptional regulatory complex was identified. It consisted of acetylating and deubiquitinating enzymatic activities (Grant et al., 1997). The SAGA deubiquitination module (DUBm) comprises of Sgf11, Sus1 and Ubp8 (Köhler et al., 2006) with corresponding human orthologs ATXN7L3, ENY2 and USP22 (as depicted in Fig. 5). H2B deubiquitination in yeast was found to be mediated by two deubiquitinating enzymes Ubp8 and Ubp10 (Henry et al., 2003);(Daniel et al., 2004)(Emre et al., 2005); (Gardner et al., 2005). Ubp8 is a part of SAGA complex and functions at promoters of SAGAdependent genes while Ubp10 is associated with non-transcribed regions regulating telomere silencing. In humans, Ubp8 homolog, USP22 was discovered (Zhao et al., 2008); (Zhang et al., 2008b) but no Ubp10 ortholog has been described till date.

Deubiquitination of H2Bub1 in humans is mainly dependent on the SAGA complex and perturbation of its subunit, ATXN7L3 leads to a great increase in the H2Bub1 levels (Lang et al., 2011). No studies have been able to establish the role of USP22 as the main DUB for H2Bub1. Some indirect studies reveal a potential link between the two. USP22 was discovered as part of 11-gene signature associated with poor prognosis determined by transcriptional profiling of tumor cells (Glinsky et al., 2005). USP22 was also shown to positively regulate p53-dependent transcription (Zhang et al., 2008b). Previously RNF20 was described as a co-activator of p53-dependent activation of p21 and MDM2 (Kim et al., 2005). Thus, it was hypothesized that an optimal balance between RNF20 and USP22 activities are required for p53dependent transcriptional activation. 


\subsection{Upstream regulation of $\mathrm{H} 2 \mathrm{Bub1}$ and transcription}

\subsubsection{Regulation via CDK9-WAC-RNF20/40 axis}

RNA polymerase II carboxyl-terminal domain (CTD) is the largest subunit of eukaryotic RNAPII consisting of a conserved 52 repeated heptapeptide in humans (YSPTSPS) (Egloff and Murphy, 2008b). Previous studies have shown that Ser5 gets phosphorylated by cyclin-dependent kinase 7 (Glover-Cutter et al., 2009) and Ser2 is phosphorylated by Positive Transcription Elongation Factor $\beta$ (P-TEFb). PTEF-b comprises of cyclin-dependent kinase 9 (CDK9) and cyclin T1 or K (Peng et al., 1998); (Fu et al., 1999). It is well established that P-Ser2 is involved in elongation, splicing and mRNA processing (Egloff and Murphy, 2008b). CDK9 plays an important role in transcription elongation. The first step for transcription elongation is Ser2 phosphorylation of RNAPII which helps in the recruitment of transcription machinery for elongation. Next, CDK9 is known to phosphorylate the factors that cause polymerase pausing, negative elongation factor-E (NELF-E) and suppressor of Ty homologue 5 (SUPT5H) subunit of DSIF. Phosphorylation of DSIF results in conversion into positive elongation factor whereas phosphorylation of NELF-E leads to disassociation from the complex. CDK9 phosphorylation events thereby promote transcription elongation (Fujinaga et al., 2004); (Yamada et al., 2006); (Peterlin and Price, 2006).

P-TEFb activity is tightly regulated in the cells. An important mechanism regulating P-TEFb is its reversible binding to HEXIM1 and 7SK snRNA which in turn inhibit the kinase activity of P-TEFb (Yik et al., 2003); (Michels et al., 2004). 7SK snRNA serves as a mediator for HEXIM1 : P-TEFb interaction. HEXIM1 and its related protein, HEXIM2 form homo or heterodimers and bind to 7SK snRNA thereby undergoing conformational change to finally bind P-TEFb (Egloff et al., 2006) (Li et al., 2005). 
Upon transcription induction or stress inducing agents, P-TEFb gets released from the HEXIM1 complex and gets recruited to the site of active transcription (Yik et al., 2003).

For transcription elongation, it was recently identified that WW domain-containing adaptor with coiled-coil protein (WAC) acts as an interaction partner of RNF20/40 complex (Zhang and Yu, 2011a). WAC interacts with P-Ser2 as well as RNF20/40 complex thereby forming a complex comprising of CDK9, WAC and RNF20/40. This provided the mechanistic link between H2Bub1 and RNAPII CTD Ser2 phosphorylation and CDK9 (Fig. 5) that had been missing for years. This finding was further substantiated by independent studies where inhibition or depletion of CDK9 led to a global decrease in H2Bub1 levels while CDK9 overexpression increased H2Bub1 (Pirngruber et al. 2009; Karpiuk et al. 2012).

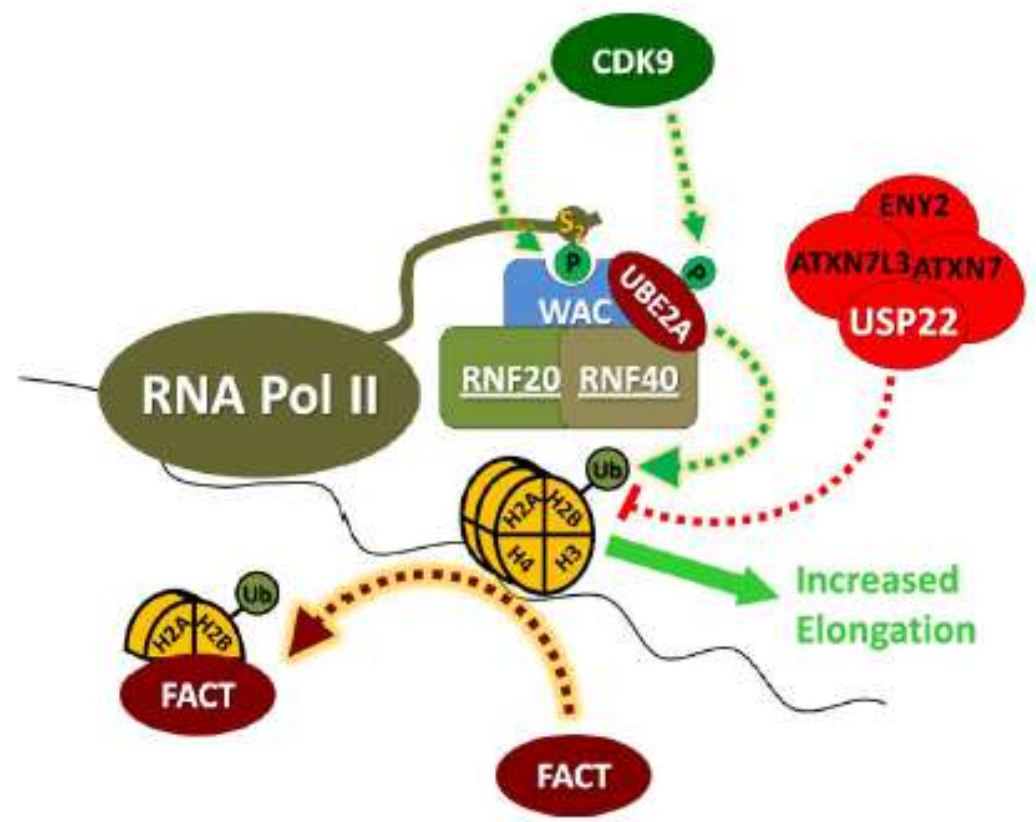

Fig. 5. The regulation of H2Bub1 through CDK9-WAC-RNF20/40 axis (Johnsen, 2012a). CDK9 phosphorylates Ser2 on the YSPTSPS heptapeptide repeat sequence on CTD of RNAPII. This recruits WW-domain containing adaptor with coiled coil protein (WAC) which further recruits the 
RNF20/RNF40 complex. This leads to monoubiquitination of histone H2B at lysine 120. H2Bub1 is deubiquitinated by the SAGA complex containing USP22, ATXN7, ATXN7L3 and ENY2.

Cancer is often associated with abnormal protein phosphorylation patterns. Under normal conditions, CDK9 was found to phosphorylate an important tumor suppressor p53 (Radhakrishnan and Gartel) and likely played a critical role as a tumor suppressor. Upon onset of malignancy, CDK9 can deregulate the p53 activation and promote tumor progression. Likewise, during AIDS, CDK9 interacted with the HIV protein Tat and enhanced viral replication and AIDS progression (Falco and Giordano) by phosphorylating RNAPII at Ser2 as well as at Ser5, while latter is normally phosphorylated by CDK7.

CDK9 and its cyclin partners are involved in the regulation of apoptosis in normal cells (Foskett et al., 2001) by promoting the expression of anti-apoptotic factors like myeloid cell leukemia 1 (MCL-1) (Chen et al., 2005). It is highly likely that deregulation of this CDK9-dependent pathway imparts the cells to resist apoptosis resulting in malignancy like it is evident in several other pathways such as EGFR (Pedersen et al., 2005).

Moreover, CDK9 was shown to interact with TNF- $\alpha$ and regulated the transcription of TNF- $\alpha$ target gene, MMP-9 thereby promoting tumor invasion and metastasis (Shan et al., 2005). There are several studies that pointed at CDK9 to play a role in several lymphomas (Bellan et al., 2004), rhabdomyosarcoma (Simone and Giordano, 2006) and prostate cancer (Lee et al., 2001).

\subsubsection{Regulation by BRD4}

Bromodomain containing protein $\underline{4}$ (BRD4), belongs to the BET (Bromodomain and Extra Terminal) family of proteins containing two tandem bromodomains at the $\mathrm{N}$ - 
terminal as well as an Extraterminal domain (ET) at the C-terminus (Florence and Faller, 2001). The family comprises of BRD 2,3,4 and testis-specific BRDT protein regulating transcription (Wu and Chiang, 2007b). Studies have shown that BRD4 interacts with CDK9 and Cyclin T1, components of P-TEFb (Positive Iranscription Elongation Factor b) (Yang et al., 2005). BRD4 binds to the P-TEFb which is free from the inhibitory complex of HEXIM1 and 7SK snRNA. BRD4 recognizes the acetylated chromatin and recruits P-TEFb which leads to CTD phosphorylation of RNA Polymerase II and thereby, BRD4 regulates transcription (Jang et al., 2005a). There were also reports that BRD4 could directly act as a kinase and phosphorylate Ser2 of RNAPII CTD and regulate transcription (Devaiah et al., 2012). The direct connection of BRD4 and H2Bub1 is not yet established but since BRD4 interacts with P-Ser2 and CDK9, it could be hypothesized that BRD4 could regulate H2Bub1 via CDK9-WAC-RNF20/40 axis.

BRD4 plays a variety of roles in several biological processes. BRD4 has been shown to regulate cell growth and BRD4 $4^{-/-}$mice are embryonic lethal (Maruyama et al., 2002) (Houzelstein et al., 2002). Unlike other bromodomain containing proteins that get released from the chromatin during mitosis, BRD4 remains bound and implicate its role in transmission of transcriptional memory across cell division (Dey et al., 2000); (Dey et al., 2003) (Kanno et al., 2004). In this way, BRD4 marks the M/G1 genes for transcription initiation in daughter cells after mitosis (Dey et al., 2009). It also functions in the inflammatory response by binding to acetylated lysine-310 residue on RelA subunit of NF-KB and acting as transcriptional co-activator of NF-KB (Huang et al., 2009). BRD4 was found to suppress HIV transcription by phosphorylating CDK9 and inhibiting its kinase activity (Zhou et al., 2009). 
Similar to CDK9, BRD4 regulated pathway is often the common target of dysregulation and results in transcriptional abnormalities.

BRD4 gene was found to be rearranged in aggressive carcinoma (French et al., 2001). BRD4 activation plays an important role in breast cancer progression (Crawford et al., 2008) and promotes epithelial to mesenchymal transition and stem cell-like conversion (Alsarraj et al., 2011b). Recently, RNA inhibitor screen identified BRD4 as an important factor for acute myeloid leukemia maintenance (Zuber et al., 2011b).

\subsubsection{Regulation by histone chaperones}

The nucleosome consists of DNA wrapped around proteins called histones. During replication or efficient transcription, the nucleosome needs to be disassembled in order to open the DNA. Likewise, after replication or transcription, DNA needs to be packaged into nucleosomes to prevent cryptic transcription. This is a cumbersome task which cannot be achieved without the class of factors called as chromatin modifiers which are further classified into two groups: ATP dependent and ATP independent. The proteins that require the energy of ATP to unwind the nucleosomes are termed as chromatin remodelers whereas the proteins that work independent of ATP energy are called histone chaperones. The nucleosome assembly is a stepwise process. Crystallographic studies have previously shown that $\mathrm{H} 3-\mathrm{H} 4$ tetramer occupies the core of the nucleosome whereas H2A-H2B tetramer binds to the peripheral region (Richmond et al., 1984). Several studies then further showed that $\mathrm{H} 3-\mathrm{H} 4$ deposition takes place first followed by $\mathrm{H} 2 \mathrm{~A}-\mathrm{H} 2 \mathrm{~B}$ and then linker histone $\mathrm{H} 1$ to form highly organized nucleosomal arrays (Smith and Stillman, 1991); (Kaufman, 1996). 
The sequential addition of histones to the chromatin is as follows: $\mathrm{H} 3-\mathrm{H} 4$ dimers are recruited stepwise to form tetramer termed as tetrasome. The $\mathrm{H} 2 \mathrm{~A}-\mathrm{H} 2 \mathrm{~B}$ dimers are then recruited in stepwise fashion to form hexasome with single $\mathrm{H} 2 \mathrm{~A}-\mathrm{H} 2 \mathrm{~B}$ dimer and then finally histone octamer after the addition of another $\mathrm{H} 2 \mathrm{~A}-\mathrm{H} 2 \mathrm{~B}$ dimer. Histone chaperones are thought to be critical for the careful incorporation of histones into an organized octamer (Das et al., 2010).

\subsubsection{Yeast SPT6 (Suppressor of Ty 6)}

SPT6 belongs to the SPT family (Suppressor of yeast transposons) of genes in Saccharomyces cerevisiae. Based on the genetic screen for mutations in yeast that affect the transcription, SPT family was found to restore the gene expression which were disrupted by the insertion of transposon Ty (Yamaguchi et al., 2001). It was shown that Spt6 along with other Spt proteins binds to RNA Pol II and affects transcription elongation (Hartzog et al., 1998). Yeast Spt6 contains SH2 domain which is responsible for its binding with RNA Pol II (Dengl et al., 2009). Spt6 was found to be $\mathrm{H} 3-\mathrm{H} 4$ chaperone due to its interaction with histone $\mathrm{H} 3$ and involved in regulating the chromatin structure (Bortvin and Winston, 1996). Moreover, it was found to regulate cryptic transcription initiation by regulating the chromatin structure after RNA Pol II passage (Kaplan et al., 2003).

\subsubsection{SUPT6H (Human homolog of yeast - Suppressor of Ty 6)}

Although a lot was known about yeast Spt6, not much had been known about the human homolog of Spt6. The human homolog, SUPT6H also contained SH2 domain and features for regulating transcription (Chiang et al., 1996). In recent past, there were discoveries that human Spt6 was also coupled with RNA Pol II and transcription elongation. SUPT6H stimulated the transcription elongation by interacting with RNA Pol II in vitro (Endoh et al., 2004). Despite the wealth of 
information, the mechanism by which SUPT6H controlled transcription elongation remains largely unknown.

As mentioned above, H2B monoubiquitination is dependent upon the activity of PTEFb and phosphorylation of Ser2 (Pirngruber et al. 2009; (Johnsen, 2012c) where the obligate heterodimeric $\mathrm{H} 2 \mathrm{~B}$ ubiquitin ligase complex RNF20/40 is linked to elongating RNAPII via the WW domain containing adaptor with coiled-coil (WAC) protein which binds directly to P-Ser2 (Zhang and Yu, 2011b). Like WAC, the histone chaperone Suppressor of Ty Homologue-6 (SUPT6H) binds to the elongating Ser2 phosphorylated form of RNAPII (Yoh et al., 2007); (Diebold et al., 2010). Thus, SUPT6H could also regulate H2Bub1 via CDK9-WAC-RNF20/40 axis. 


\subsection{Aim of the project}

H2Bub1 is important for several processes such as gene expression, DNA damage and cellular differentiation. However, H2Bub1 is a mark for execution of these events but what dictates the placement of this mark needs further investigation. Thus, the aim of this study was to investigate the role of H2Bub1 and its upstream regulators, histone chaperone SUPT6H and bromodomain protein BRD4 in breast cancer. To study the effect of these regulators, SUPT6H was depleted and the influence on ERa-dependent signaling, critical for breast cancer was examined. High-throughput RNA and ChIP-sequencing was used to study the regulation by BRD4 and H2Bub1 in suppression of stem cell-like phenotype in mammary cells. 


\section{Materials \& Methods}

\subsection{Technical equipment}

\begin{tabular}{|c|c|}
\hline Agarose gel chamber & Harnischmacher Labortechnik, Kassel \\
\hline Balance & Sartorius AG, Göttingen \\
\hline Bandelin Sonoplus Sonicator & Bandelin electr. GmbH \& Co. KG, Berlin \\
\hline Biological Safety Cabinet "Hera Safe" & Thermo Fisher Scientific, Waltham, USA \\
\hline Bioruptor Plus & Diagenode, Belgium, Europe \\
\hline Centrifuge (Megafuge 1.OR) & Thermo Fisher Scientific, Waltham, USA \\
\hline Centrifuge $4^{\circ} \mathrm{C}(5417 \mathrm{R})$ & Eppendorf AG, Hamburg \\
\hline C1000TM Thermal Cycler & Bio-Rad Laboratories GmbH, München \\
\hline CFX96TM Optical Reaction Module & Bio-Rad Laboratories GmbH, München \\
\hline Counting chamber (Neubauer) & Brand GmbH \& Co. KG, Wertheim \\
\hline Confocal microscope LSM510 META & Carl Zeiss Microlmaging GmbH, Göttingen \\
\hline 5100 Cryo $1^{\circ} \mathrm{C}$ Freezing Container & Thermo Fisher Scientific \\
\hline Electrophoresis \& Electrotransfer Unit & Bio-Rad Laboratories GmbH, München \\
\hline Freezer $-20^{\circ} \mathrm{C}$ & Liebherr GmbH, Biberach \\
\hline Freezer $-80^{\circ} \mathrm{C}$ "Hera freeze" & Thermo Fisher Scientific, Waltham, USA \\
\hline Gel Imager "Gel iX imager" & Intas Science Imaging GmbH, Göttingen \\
\hline Incubator (bacteria) & Memmert GmbH \& Co. KG, Schwabach \\
\hline Incubator (bacteria culture) & Infors AG, Bottmingen \\
\hline Incubator (cell culture) "Hera cell 150" & Thermo Fisher Scientific, Waltham, USA \\
\hline Inverse Microscope "Axiovert 40 CFL" & $\begin{array}{l}\text { Carl Zeiss Microlmaging GmbH, } \\
\text { Göttingen }\end{array}$ \\
\hline Luminometer 2030-100 & Turner designs, Sunnyvale, CA, USA \\
\hline Magnet stirrer "MR3001" & Heidolph GmbH \& Co. KG, Schwabach \\
\hline Microscope "Axiovert 40 C" & Carl Zeiss Microlmaging GmbH, Göttingen \\
\hline Microwave & Clatronic International GmbH, Kempen \\
\hline Nano Drop® ND-1000 & Peqlab Biotechnology GmbH, Erlangen \\
\hline
\end{tabular}




\begin{tabular}{|l|l|}
\hline Spectrophotometer & \\
\hline Pestle & Sartorius AG, Göttingen \\
\hline pH meter & noLab® WTW GmbH, Weilheim \\
\hline Pipette Aid® portable XP & $\begin{array}{l}\text { Drummond Scientific Co., Broomall, } \\
\text { USA }\end{array}$ \\
\hline Pipettes "Research" Series & Eppendorf AG, Hamburg \\
\hline Power supply “Power Pack P25T" & Biometra GmbH, Göttingen Material \\
\hline Qubit® 2.0 Fluorometer & Invitrogen GmbH, Karlsruhe \\
\hline Refrigerator & Liebherr GmbH, Biberach \\
\hline Repeat Pipette & Gilson Inc., Middleton, USA \\
\hline ScanScope XT & Aperio, Vista, CA, USA \\
\hline Scanner (CanoScan 8600F) & Canon GmbH, Krefeld \\
\hline Shaker "Rocky" & Schütt Labortechnik GmbH, Göttingen \\
\hline Table centrifuge (GMC-060) & LMS Co., Ltd., Tokyo, Japan \\
\hline Test tube rotator & Schütt Labortechnik GmbH, Göttingen \\
\hline Ultrapure Water System “Aquintus" & MembraPure GmbH, Bodenheim \\
\hline Vacuum pump & $\begin{array}{l}\text { Integra Bioscienc. AG, Zizers, } \\
\text { Switzerland }\end{array}$ \\
\hline Vortex mixer & Scientific Industries, Inc., Bohemia, USA \\
\hline Water bath “TW 20" & JULABO Labortechnik GmbH, Seelbach \\
\hline X- Ray Cassettes & Rego X-ray GmbH. Augsburg \\
\hline & \\
\hline
\end{tabular}

\subsection{Consumable materials}

\begin{tabular}{|l|l|}
\hline Cellstar 6- and 12-well cell culture plate & Greiner Bio-One GmbH, Frickenhausen \\
\hline Cellstar PP-tube 15 and $50 \mathrm{ml}$ & Greiner Bio-One $\mathrm{GmbH}$, Frickenhausen \\
\hline Cellstar tissue culture dish $100 \times 20 \mathrm{~mm}$ & Greiner Bio-One GmbH, Frickenhausen \\
\hline Cellstar tissue culture dish $145 \times 20 \mathrm{~mm}$ & Greiner Bio-One GmbH, Frickenhausen \\
\hline Cell scraper $(16 \mathrm{~cm}, 25 \mathrm{~cm})$ & Sarstedt AG \& Co., Nümbrecht \\
\hline Cryo TubeTM Vial $(1.8 \mathrm{ml})$ & Thermo Fisher Scientific, Waltham, USA \\
\hline
\end{tabular}




\begin{tabular}{|l|l|}
\hline Falcon® assay plate, 96 well & VWR Int., LLC, West Chester, USA \\
\hline Gel blotting paper (Whatman paper) & Sartorius AG, Göttingen \\
\hline Glass coverslips (18 mm) & Gebr. Rettberg GmbH, Göttingen \\
\hline HybondTM-PVDF Transfer Membrane & GE Healthcare Europe GmbH, München \\
\hline Microtube 0,5 ml, $1.5 \mathrm{ml}, 2 \mathrm{ml}$ & Sarstedt AG \& Co., Nümbrecht \\
\hline Microtube 1.5 ml, conical & VWR International GmbH, Darmstadt \\
\hline 96 Multiply® PCR plate white & Sarstedt AG \& Co., Nümbrecht \\
\hline 96-well Multiplate PCR plate white (low) & Bio-Rad Laboratories GmbH, München \\
\hline $\begin{array}{l}\text { NORM-JECT Syringes of different } \\
\text { volume }\end{array}$ & Henke Sass Wolf GmbH, Tuttlingen \\
\hline Parafilm® "M" & Pechiney Plastic Packaging, Chicago, USA \\
\hline PET track-etched cell culture inserts & BD Bioscience, Franklin Lakes, NJ, USA \\
\hline Petri dish 92×16 mm & Sarstedt AG \& Co., Nümbrecht \\
\hline Pipette tips & Greiner Bio-One GmbH, Frickenhausen \\
\hline Pipette filter tips & Sarstedt AG \& Co., Nümbrecht \\
\hline $\begin{array}{l}\text { Protan® Nitrocellulose transfer } \\
\text { membrane }\end{array}$ & Whatman GmbH, Dassel \\
\hline Shandon Coverplate & Thermo Fisher Scientific, Waltham, USA \\
\hline Syringe filter, Ca-membrane, 0,20 m & Sartorius AG, Göttingen \\
\hline Tissue microarrays US & Biomax,Inc., Rockville, MD, USA \\
\hline Ultra low attachment plates & Corning Life sciences, NY, USA \\
\hline X-ray films “Super RX" & Fujifilm Corp., Tokyo, Japan \\
\hline
\end{tabular}

\subsection{Chemicals}

\subsubsection{General chemicals}

\begin{tabular}{|l|l|}
\hline Acetic acid & Carl Roth GmbH \& Co. KG, Karlsruhe \\
\hline Adefodur WB developing concentrate & Adefo-Chemie GmbH, Dietzenbach \\
\hline Adefodur WB fixing concentrate & Adefo-Chemie GmbH, Dietzenbach \\
\hline Agarose & Biozym Scientific GmbH, Oldendorf \\
\hline Albumin Fraction V (BSA) & Carl Roth GmbH \& Co. KG, Karlsruhe \\
\hline Ammonium persulfate (APS) & Carl Roth GmbH \& Co. KG, Karlsruhe \\
\hline
\end{tabular}




\begin{tabular}{|l|l|}
\hline Ammonium sulfate $\left(\mathrm{NH}_{4}\right)_{2} \mathrm{SO}_{4}$ & Carl Roth GmbH \& Co. KG, Karlsruhe \\
\hline Aprotinin & Carl Roth GmbH \& Co. KG, Karlsruhe \\
\hline Bromophenol blue & Sigma-Aldrich Co., St. Louis, USA \\
\hline Calcium Chloride $\left(\mathrm{CaCl}_{2}\right)$ & Carl Roth GmbH \& Co. KG, Karlsruhe \\
\hline Charcoal Dextran treated FBS & Thermo Scientific HyClone, Logan, USA \\
\hline Chelex (Chelating lon Exchange Resin) & Bio-Rad Laboratories GmbH, München \\
\hline Chloroform & Carl Roth GmbH \& Co. KG, Karlsruhe \\
\hline Cholera Toxin & Sigma-Aldrich Co., St. Louis, USA \\
\hline Co-precipitant Pink & Bioline, Luckenwalde \\
\hline Colorless co-precipitant & Bioline, Luckenwalde \\
\hline Crystal violet & Sigma-Aldrich Co., St. Louis, USA \\
\hline Citric acid & Carl Roth GmbH \& Co. KG, Karlsruhe \\
\hline DePeX mounting media & VWR International GmbH \\
\hline Diaminobenzidine substrate & ImmPACTTM DAB, SK-4105, Vector, \\
\hline Biethylpyrocarbonate (DEPC) & Carl Roth GmbH \& Co. KG, Karlsruhe \\
\hline Dimethyl sulfoxide (DMSO) & AppliChem GmbH, Darmstadt \\
\hline Dithiothreitol (DTT) & Carl Roth GmbH \& Co. KG, Karlsruhe \\
\hline DMEM & GlBCO®, Invitrogen GmbH, Darmstadt \\
\hline DMEM/F12 & GlBCO®, Invitrogen GmbH, Darmstadt \\
\hline dNTPs & Carl Roth GmbH \& Co. KG, Karlsruhe \\
\hline Ethanol absolute & Th. Geyer GmbH \& Co. KG, Renningen \\
\hline Ethidium bromide & Carl Roth GmbH \& Co. KG, Karlsruhe \\
\hline Ethylenediaminetetraacetic acid (EDTA) & Carl Roth GmbH \& Co. KG, Karlsruhe \\
\hline Epidermal Growth Factor (EGF) & Sigma-Aldrich Co., St. Louis, USA \\
\hline Fetal Bovine Serum (FBS) & Thermo Scientific HyClone, Logan, USA \\
\hline Formaldehyde & Sigma-Aldrich Co., St. Louis, USA \\
\hline Glycerol & Carl Roth GmbH \& Co. KG, Karlsruhe \\
\hline B-Glycerolphosphate (BGP) & Sigma-Aldrich Co., St. Louis, USA \\
\hline Glycine & Merck, Darmstadt \\
\hline GlycoBlue & \\
\hline Hematoxyline & GmbH \& Co. KG, Karlsruhe \\
\hline
\end{tabular}




\begin{tabular}{|l|l|}
\hline Horse Serum & Sigma-Aldrich Co., St. Louis, USA \\
\hline Hydrochloric acid (HCl) & Carl Roth GmbH \& Co. KG, Karlsruhe \\
\hline Hydrocortisone & Sigma-Aldrich Co., St. Louis, USA \\
\hline Insulin & Sigma-Aldrich Co., St. Louis, USA \\
\hline lodacetamide & Sigma-Aldrich Co., St. Louis, USA \\
\hline Isopropanol & Carl Roth GmbH \& Co. KG, Karlsruhe \\
\hline Leupeptin & Carl Roth GmbH \& Co. KG, Karlsruhe \\
\hline Lithium chloride (LiCl), 8M & Sigma-Aldrich Co., St. Louis, USA \\
\hline Magnesium chloride (MgCl $\left.{ }_{2}\right)$ & Carl Roth GmbH \& Co. KG, Karlsruhe \\
\hline MEM, no Glutamine, No Phenol Red & Life Technologies, Carlsbad, USA \\
\hline Methanol & M. Baker B.V., Deventer, Netherlands \\
\hline N-ethylmaleimide (NEM) & Sigma-Aldrich Co., St. Louis, USA \\
\hline Nickel chloride (NiCl $\left.{ }_{2}\right)$ & Sigma-Aldrich Co., St. Louis, USA \\
\hline Nile Red & Sigma-Aldrich Co., St. Louis, USA \\
\hline NonidetTM P40 (NP-40) & Sigma-Aldrich Co., St. Louis, USA \\
\hline Opti-MEM & GlBCO®, Invitrogen GmbH, Darmstadt \\
\hline PBS tablets & GlBCO®, Invitrogen GmbH, Darmstadt \\
\hline Pefabloc SC Protease Inhibitor & Carl Roth GmbH \& Co. KG, Karlsruhe \\
\hline Penicillin-Streptomycin solution & Sigma-Aldrich Co., St. Louis, USA \\
\hline Peptone & Carl Roth GmbH \& Co. KG, Karlsruhe \\
\hline Potassium acetate & Carl Roth GmbH \& Co. KG, Karlsruhe \\
\hline Potassium chloride (KCl) & AppliChem GmbH, Darmstadt \\
\hline Potassium dihydrogen phosphate & Carl Roth GmbH \& Co. KG, Karlsruhe \\
\hline Protein-A Sepharose CL-4B & GE Healthcare, Uppsala, Sweden \\
\hline Protein-G Sepharose 4 Fast Flow & GE Healthcare, Uppsala, Sweden \\
\hline RNase inhibitor & New England Biolabs, Frankfurt am Main \\
\hline RNAiMAX & Invitrogen GmbH, Karlsruhe \\
\hline Roti®-Phenol & Carl Roth GmbH \& Co. KG, Karlsruhe \\
\hline Rotiphorese® Gel 30 & Carl Roth GmbH \& Co. KG, Karlsruhe \\
\hline Rotipuran® Chloroform & Carl Roth GmbH \& Co. KG, Karlsruhe \\
\hline Rotipuran® Isoamylalcohol & \\
\hline
\end{tabular}




\begin{tabular}{|l|l|}
\hline SepharoseTM CL-4B & GE Healthcare, Uppsala, Sweden \\
\hline Skim milk powder & Carl Roth GmbH \& Co. KG, Karlsruhe \\
\hline Sodium acetate & Carl Roth GmbH \& Co. KG, Karlsruhe \\
\hline Sodium azide & AppliChem GmbH, Darmstadt \\
\hline Sodium chloride (NaCl) & Carl Roth GmbH \& Co. KG, Karlsruhe \\
\hline Sodium deoxycholate & AppliChem GmbH, Darmstadt \\
\hline Sodium dodecylsulfate (SDS) & Carl Roth GmbH \& Co. KG, Karlsruhe \\
\hline Sodium fluoride (NaF) & AppliChem GmbH, Darmstadt \\
\hline di-Sodium hydrogen phosphate & Carl Roth GmbH \& Co. KG, Karlsruhe \\
\hline Sodium hydroxide (NaOH) & Carl Roth GmbH \& Co. KG, Karlsruhe \\
\hline Sodium pyruvate (Na-Pyr) & GlBCO®, Invitrogen GmbH, Darmstadt \\
\hline SYBR Green I & Roche Diagnostics GmbH, Mannheim \\
\hline TEMED & Carl Roth GmbH \& Co. KG, Karlsruhe \\
\hline a,a-Trehalose Dihydrate & USB Corporation, Cleveland, USA \\
\hline Tris & Carl Roth GmbH \& Co. KG, Karlsruhe \\
\hline Triton X-100 & AppliChem GmbH, Darmstadt \\
\hline TRIzol® Reagent & Invitrogen GmbH, Karlsruhe \\
\hline Trypsin-EDTA (0.05\%) & GlBCO®, Invitrogen GmbH, Darmstadt \\
\hline Tween-20 & AppliChem GmbH, Darmstadt \\
\hline Xylene & Carl Roth GmbH \& Co. KG, Karlsruhe \\
\hline & \\
\hline
\end{tabular}

\subsubsection{Differentiation chemicals}

\begin{tabular}{|l|l|}
\hline Ascorbic acid & Sigma-Aldrich Co., St. Louis, USA \\
\hline Calcitriol (1a,25-dihydroxy Vitamin D3) & Cayman chemicals, Ann Arbor, USA \\
\hline Dexamethasone & Sigma-Aldrich Co., St. Louis, USA \\
\hline B-Glycerolphosphate (BGP) & Sigma-Aldrich Co., St. Louis, USA \\
\hline Isobuthylmetylxantine (IBMX) & Sigma-Aldrich Co., St. Louis, USA \\
\hline Troglitazone & Sigma-Aldrich Co., St. Louis, USA \\
\hline
\end{tabular}




\subsection{Kits and reagents}

\begin{tabular}{|l|l|}
\hline Alkaline phosphatase leukocyte kit & Sigma-Aldrich Co., St. Louis, USA \\
\hline Bioanalyzer DNA High sensitivity kit & Agilent, Santa Clara, USA \\
\hline $\begin{array}{l}\text { Immobilon Western Chemiluminescent } \\
\text { HRP Substrate }\end{array}$ & Millipore, Billerica, USA \\
\hline LipofectamineTM 2000 & Invitrogen GmbH, Karlsruhe \\
\hline LipofectamineTM RNAiMAX & Invitrogen GmbH, Karlsruhe \\
\hline PureYieldTM Plasmid Midiprep & Promega GmbH, Mannheim \\
\hline QIAprep® Spin Miniprep Kit & Qiagen GmbH, Hilden \\
\hline Qubit dsDNA HS assay & Invitrogen GmbH, Karlsruhe \\
\hline SuperSignal® West Dura & Thermo Fisher Scientific, Waltham, USA \\
\hline SuperSignal® West Femto Maximum & Thermo Fisher Scientific, Waltham, USA \\
\hline
\end{tabular}

\subsection{Nucleic acids}

\subsection{1 siRNA Oligonucleotides}

Target Gene siRNA Target Sequence Source

\begin{tabular}{|c|c|c|c|}
\hline Target Gene & siRNA sequence 5'-3' direction & Source & Cat. No. \\
\hline $\begin{array}{c}\text { siGENOME Nontargeting } \\
\text { siRNA pool \# 1 }\end{array}$ & --- & Dharmacon & D-001206-13 \\
\hline CD24 siGENOME (\# 5) & GAGCAAUGGUGGCCAGGCU & Dharmacon & D-187156-05 \\
\hline CD24 siGENOME (\# 6) & GCAGAUUUAUUCCAGUGAA & Dharmacon & D-187156-06 \\
\hline CD24 siGENOME (\# 7) & CAACUAAUGCCACCACCAA & Dharmacon & D-187156-07 \\
\hline CD24 siGENOME (\# 8) & GGUGGUGCCCUGCAGUCAA & Dharmacon & D-187156-08 \\
\hline RNF40 siGENOME (\# 1) & GAGAUGCGCCACCUGAUUAUU & Dharmacon & D-006913-01 \\
\hline RNF40 siGENOME (\# 2) & GAUGCCAACUUUAAGCUAAUU & Dharmacon & D-006913-02 \\
\hline RNF40 siGENOME (\# 3) & GAUCAAGGCCAACCAGAUUUU & Dharmacon & D-006913-03 \\
\hline RNF40 siGENOME (\# 4) & CAACGAGUCUCUGCAAGUGUU & Dharmacon & D-006913-04 \\
\hline RNF20 siGENOME (\#1) & CCAAUGAAAUCAAGUCUAA & Dharmacon & D-007027-01 \\
\hline RNF20 siGENOME (\#2) & UAAGGAACUCCAGAAUAU & Dharmacon & D-007027-02 \\
\hline
\end{tabular}




\begin{tabular}{|c|c|c|c|}
\hline RNF20 siGENOME $(\# 3)$ & GCAAAUGUCCCAAGUGUAA & Dharmacon & D-007027-03 \\
\hline RNF20 siGENOME $(\# 4)$ & AGAAGAAGCUACAUGAUUU & Dharmacon & D-007027-04 \\
\hline BRD4 siGENOME $(\# 2)$ & GAACCUCCCUGAUUACUAU & Dharmacon & D-004937-02 \\
\hline BRD4 siGENOME $(\# 3)$ & UAAAUGAGCUACCCACAGA & Dharmacon & D-004937-03 \\
\hline BRD4 siGENOME $(\# 4)$ & UGAGAAAUCUGCCAGUAAU & Dharmacon & D-004937-04 \\
\hline BRD4 siGENOME $(\# 5)$ & AGCUGAACCUCCCUGAUUA & Dharmacon & D-004937-05 \\
\hline SUPT6H siGENOME $(\# 1)$ & GAACAUGACUUCACAGAUG & Dharmacon & D-010540-01 \\
\hline SUPT6H siGENOME $(\# 3)$ & CCAGAGACCUUCUACAUUG & Dharmacon & D-010540-03 \\
\hline SUPT6H siGENOME $(\# 4)$ & AAAGAAGGCUCAAGACAUU & Dharmacon & D-010540-04 \\
\hline SUPT6H siGENOME $(\# 18)$ & GGGGAGAACCUGCGGGAUA & Dharmacon & D-010540-18 \\
\hline
\end{tabular}

For transfections, the Dharmacon siRNAs (\#1 - \#4) were pooled in a 1:1:1:1 ratio.

\subsubsection{RT PCR primers}

Reverse Transcription primers were ordered from Metabion AG, Martinisried and Sigma Aldrich, Hamburg.

\subsubsection{Quantitative PCR primers (qPCR)}

Primers mentioned were used in 5' to 3' orientation and designed using the NCBI primer designing tool (http://www.ncbi.nlm.nih.gov/tools/primer-blast/).

\begin{tabular}{|c|c|c|c|}
\hline Gene & Forward primer (5' to 3') & Reverse primer (5' to 3') & Reference \\
\hline 18S rRNA & AACTGAGGCCATGATTAA & GGAACTACGACGGTATCTGA & This study \\
\hline ACTA2 & ACCTTTGGCTTGGCTTGTCA & GGAAGCTTTAGGGTCGCTGG & This study \\
\hline AXL & ATCGCCAAGATGCCAGTCAA & CACATTGTCACCCCGAAGG & This study \\
\hline CK19 & GAATCGCAGCTTCTGAGACCA & CTGGCGATAGCTGTAGGAAGTC & This study \\
\hline CXCL12 & TGCCAGAGCCAACGTCAAGCATC & CGGGTCAATGCACACTTGTCTGTTGT & $\begin{array}{c}\text { (Prenzel et } \\
\text { al., 2011) }\end{array}$ \\
\hline DLL1 & GCAAGCGTGACACCAAGTG & AAGTTGAACAGCCCGAGTCC & This study \\
\hline FN1 & CCCTGGTGTCACAGAGGCTA & GAGAGAGAGCTTCTTGTCCTGTC & This study \\
\hline GAS6 & ACCTGACCGTGGGAGGTATT & GTGTCTTCTCCGTTCAGCCA & This study \\
\hline
\end{tabular}




\begin{tabular}{|c|c|c|c|}
\hline GREB1 & GTGGTAGCCGAGTGGACAAT & ATTTGTTTCCAGCCCTCCTT & $\begin{array}{c}\text { (Prenzel et } \\
\text { al., 2011) }\end{array}$ \\
\hline HNRNPK & ATCCGCCCCTGAACGCCCAT & ACATACCGCTCGGGGCCACT & $\begin{array}{l}\text { (Karpiuk et } \\
\text { al., 2012a) }\end{array}$ \\
\hline IL32 & AGAAGCTGAAGGCCCGAATG & TGTCCACGTCCTGATTCTGC & This study \\
\hline NFKB2 & CTACTGGAGGCCCTGTCTGA & CCGTACGCACTGTCTTCCTT & This study \\
\hline NFKBIZ & GATGCTGTCCGCCTGTTGAT & CACTGGCTGTTCGTTCTCCA & This study \\
\hline PGR & TCCACCCCGGTCGCTGTAGG & TAGAGCGGGCGGCTGGAAGT & $\begin{array}{c}\text { (Prenzel et } \\
\text { al., 2011) }\end{array}$ \\
\hline PPARG & ACCTCCGGGCCCTGGCAAAA & TGCTCTGCTCCTGCAGGGGG & $\begin{array}{l}\text { (Karpiuk et } \\
\text { al., 2012a) }\end{array}$ \\
\hline RELB & AGCGGAAGATTCAACTGGGC & TGTCATAGACGGGCTCGGAA & This study \\
\hline RNF40 & AGTACAAGGCGCGGTTGA & GAAGCAGAAAACGTGGAAGC & $\begin{array}{c}\text { (Prenzel et } \\
\text { al., 2011) }\end{array}$ \\
\hline ROR1 & AAACGGCAAGGAGGTGGTTT & TGCACATGCAATCCСTCTGT & This study \\
\hline RUNX2 & GCGGTGCAAACTTTCTCCAG & GCAGCCTTAAATGACTCTGTTGG & This study \\
\hline SERPINE1 & GACCTCAGGAAGCCCCTAGA & GTGCCACTCTCGTTCACCTC & This study \\
\hline SLIT2 & TTCACCTCTTCGGGCCATTC & AGCCACTTGAGATGGCAGTC & This study \\
\hline SUPT6H & GAAAACGCACCTCTTTTGATG & CGTCCTCGTCATCTGACATTT & This study \\
\hline TGFBR2 & TCGCTGTAATGCAGTGGGAG & TCATGCTTTCGACACAGGGG & This study \\
\hline TJP3 & CAGAGCATGGAGGATCGTGG & TCAGGTTCTGGAATGGCACG & This study \\
\hline TNFSF10 & TGCGTGCTGATCGTGATCTT & GCATCTGCTTCAGCTCGTTG & This study \\
\hline TNFSF12 & CTGGGAGGAAGCCAGAATCAAC & TCATCAAAGTGCACCTGACAGTA & This study \\
\hline
\end{tabular}

\subsubsection{ChIP primers}

\begin{tabular}{|c|c|c|c|}
\hline Gene & Forward primer (5' to $\left.3^{\prime}\right)$ & Reverse primer (5' to $\left.3^{\prime}\right)$ & Reference \\
\hline CXCL12 TSS & GCAGTGCGCTCCGGCCTTT & ССTCACTGCAGACCGGGCCA & $\begin{array}{c}\text { (Prenzel et al., } \\
\text { 2011) }\end{array}$ \\
\hline CXCL12 TR & AAAGAGCCTGTCTGCAGGTG & CCTGTCTCTTCTCGGGTTCAC & This study \\
\hline GAPDH TSS & CGGCTACTAGCGGTTTTACG & AAGAAGATGCGGCTGACTGT & This study \\
\hline GREB1 ERE & CCTGGGAATGGAGATTTTGATA & GAGCTGCGAGTCCCTAACAG & $\begin{array}{c}\text { (Prenzel et al., } \\
\text { 2011) }\end{array}$ \\
\hline GREB1 TSS & GCCAAATGGAAGAAGGACAG & АССАССТАССТССАGTСАСС & $\begin{array}{c}\text { (Prenzel et al., } \\
2011)\end{array}$ \\
\hline GREB1 TR & AGTGCAGGGAGAAAGGCAAG & GGAGAGCATGGTGTGCAGAT & This study \\
\hline PDK4 BV & GCGTCGAGGCTCCAGGGCT & GCCCAAGCTGGGTCCTAGGGTT & $\begin{array}{c}\text { (Karpiuk et al., } \\
\text { 2012a) }\end{array}$ \\
\hline PGR ERE & GGCCAGCAGTCCTGCAACAGTC & CCCAAGCTTGTCCGCAGCCTT & $\begin{array}{c}\text { (Prenzel et al., } \\
\text { 2011) }\end{array}$ \\
\hline PGR TSS & GTGCGTGTGGGTGGCATTCTC & GCGGGAGCACTAGCCGCC & This study \\
\hline
\end{tabular}




\begin{tabular}{|c|c|c|c|}
\hline PGR TR & AGTCCGCTGTCCTTTTCTGG & TATCTCCCTGGACGGGCTAC & This study \\
\hline PPARG BV & AGCCGCTCCGGGGGAACTT & ACAGGGCCTGGCCAGCTACAA & $\begin{array}{c}\text { (Karpiuk et al., } \\
\text { 2012a) }\end{array}$ \\
\hline RASD1 BV & CGGCCACCCTCACCTTCTCCT & GATCTGCTGCCTGAGCCGCTG & $\begin{array}{c}\text { (Karpiuk et al., } \\
\text { 2012a) }\end{array}$ \\
\hline TFF1 TSS & CCTGGATTAAGGTCAGGTTGGA & TCTTGGCTGAGGGATCTGAGA & $\begin{array}{c}\text { (Prenzel et al., } \\
\text { 2011) }\end{array}$ \\
\hline TFF1 TR & CCACTCCCTAGAAGGACCCA & GCTGGCAACCCATATTCCCT & This study \\
\hline
\end{tabular}

\subsubsection{Primers for ChIP-seq Library preparation}

\begin{tabular}{|c|c|c|c|}
\hline $\begin{array}{c}\text { Protein/ } \\
\text { Modification }\end{array}$ & Primer & $5 '-3$ ' Sequence & Reference \\
\hline & $\begin{array}{l}\text { Multi- } \\
\text { Adap1 F }\end{array}$ & Phosphate-GATCGGAAGAGCACACGTCT & Illumina \\
\hline & $\begin{array}{c}\text { Multi- } \\
\text { Adapt1 R }\end{array}$ & АCACTCTTTCCCTACACGACGCTCTTCCGATC*T & Illumina \\
\hline & $\begin{array}{l}\text { Multi-PCR- } \\
1.0\end{array}$ & $\begin{array}{l}\text { AATGATACGGCGACCACCGAGATCTACACTCTTTCC } \\
\text { CTACACGACGCTCTTCCGATC*T }\end{array}$ & $\begin{array}{l}\text { Modified from } \\
\text { Illumina }\end{array}$ \\
\hline BRD4 -1 & $\begin{array}{l}\text { Multi-PCR- } \\
\text { Ind5 }\end{array}$ & $\begin{array}{c}\text { CAAGCAGAAGACGGCATACGAGATCACTGTGTGAC } \\
\text { TGGAGTTCAGACGTGTGCTCTTCCGATC*T }\end{array}$ & $\begin{array}{l}\text { Modified from } \\
\text { Illumina }\end{array}$ \\
\hline BRD4 - 2 & $\begin{array}{l}\text { Multi-PCR- } \\
\text { Ind6 }\end{array}$ & $\begin{array}{l}\text { CAAGCAGAAGACGGCATACGAGATATTGGCGTGAC } \\
\text { TGGAGTTCAGACGTGTGCTCTTCCGATC*T }\end{array}$ & $\begin{array}{l}\text { Modified from } \\
\text { Illumina }\end{array}$ \\
\hline BRD4 - 3 & $\begin{array}{l}\text { Multi-PCR- } \\
\text { Ind12 }\end{array}$ & $\begin{array}{l}\text { CAAGCAGAAGACGGCATACGAGATTACAAGGTGAC } \\
\text { TGGAGTTCAGACGTGTGCTCTTCCGATC*T }\end{array}$ & $\begin{array}{l}\text { Modified from } \\
\text { Illumina }\end{array}$ \\
\hline H2Bub1 - 1 & $\begin{array}{l}\text { Multi-PCR- } \\
\text { Ind7 }\end{array}$ & $\begin{array}{l}\text { CAAGCAGAAGACGGCATACGAGATGATCTGGTGAC } \\
\text { TGGAGTTCAGACGTGTGCTCTTCCGATC*T }\end{array}$ & $\begin{array}{l}\text { Modified from } \\
\text { Illumina }\end{array}$ \\
\hline H2Bub1 - 2 & $\begin{array}{l}\text { Multi-PCR- } \\
\text { Ind9 }\end{array}$ & $\begin{array}{l}\text { CAAGCAGAAGACGGCATACGAGATCTGATCGTGAC } \\
\text { TGGAGTTCAGACGTGTGCTCTTCCGATC*T }\end{array}$ & $\begin{array}{l}\text { Modified from } \\
\text { Illumina }\end{array}$ \\
\hline H2Bub1 - 3 & $\begin{array}{l}\text { Multi-PCR- } \\
\text { Ind10 }\end{array}$ & $\begin{array}{l}\text { CAAGCAGAAGACGGCATACGAGATAAGCTAGTGAC } \\
\text { TGGAGTTCAGACGTGTGCTCTTCCGATC*T }\end{array}$ & $\begin{array}{l}\text { Modified from } \\
\text { Illumina }\end{array}$ \\
\hline H3K27ac - 1 & $\begin{array}{l}\text { Multi-PCR- } \\
\text { Ind20 }\end{array}$ & $\begin{array}{c}\text { CAAGCAGAAGACGGCATACGAGATGGCCACGTGAC } \\
\text { TGGAGTTCAGACGTGTGCTCTTCCGATC*T }\end{array}$ & $\begin{array}{l}\text { Modified from } \\
\text { Illumina }\end{array}$ \\
\hline H3К27ac - 2 & $\begin{array}{l}\text { Multi-PCR- } \\
\text { Ind23 }\end{array}$ & $\begin{array}{l}\text { CAAGCAGAAGACGGCATACGAGATCCACTCGTGAC } \\
\text { TGGAGTTCAGACGTGTGCTCTTCCGATC*T }\end{array}$ & $\begin{array}{l}\text { Modified from } \\
\text { Illumina }\end{array}$ \\
\hline
\end{tabular}

\subsection{Proteins}

\subsubsection{Molecular weight standards}

Gene RulerTM DNA-Ladder

PageRulerTM Prestained Protein Ladder
Fermentas GmbH, St. Leon-Rot

Fermentas $\mathrm{GmbH}$, St. Leon-Rot 


\subsubsection{Enzymes}

\begin{tabular}{|l|l|}
\hline Phusion polymerase & New England Biolabs, Frankfurt am Main \\
\hline Proteinase K & Invitrogen GmbH, Karlsruhe \\
\hline Restriction enzymes & New England Biolabs, Frankfurt am Main \\
\hline Reverse Transcriptase (M-MuLV) & New England Biolabs, Frankfurt am Main \\
\hline RNase A & Qiagen GmbH, Hilden \\
\hline Taq DNA Polymerase & Prime Tech, Mink, Belarus \\
\hline T4 DNA Ligase & New England Biolabs, Frankfurt am Main \\
\hline
\end{tabular}

\subsubsection{Antibodies}

\subsubsection{Primary antibodies}

Following antibodies were used for ChIP and Western blot analyses in the mentioned dilutions.

\begin{tabular}{|c|c|c|c|c|c|c|c|}
\hline Name & Clone & Cat. No. & WB & ChIP & IF & IHC & Source \\
\hline BRD4 & (N-term) & T2948 & $1: 2000$ & & & & Epitomics \\
\hline BRD4 & (N-term) & & & $1 \mu \mathrm{g}$ & & & (Wu et al., 2006) \\
\hline CK8/18 & & BP-5007 & & & $1: 200$ & & Acris \\
\hline $\begin{array}{c}\text { E- } \\
\text { Cadherin }\end{array}$ & 24E10 & 3195 & $1: 2000$ & & & & Cell Signaling \\
\hline ERa & & sc-543 & $1: 1000$ & $1 \mu \mathrm{g}$ & & & Santa Cruz \\
\hline $\mathrm{H} 2 \mathrm{~B}$ & - & $07-371$ & $1: 3000$ & - & & & Millipore \\
\hline H2Bub1 & 56 & 05-1312 & $1: 5000$ & $2 \mu g$ & & & Millipore (for WB) \\
\hline H2Bub1 & 7B4 & & & & $1: 200$ & & $\begin{array}{c}\text { (Prenzel et al., } \\
2011)\end{array}$ \\
\hline H2Bub1 & D11 & 5546 & - & $1 \mu l$ & & & Cell Signaling \\
\hline H3K4me3 & & $\begin{array}{c}\text { MAb-152- } \\
050\end{array}$ & & $1 \mu \mathrm{g}$ & & & Diagenode \\
\hline H3K27me3 & & $\begin{array}{c}\text { pAb-069- } \\
050\end{array}$ & & $1 \mu \mathrm{g}$ & & & Diagenode \\
\hline HSC70 & B-6 & sc-7298 & $1: 25000$ & - & & & Santa Cruz \\
\hline $\begin{array}{l}\text { IgG (non- } \\
\text { specific) }\end{array}$ & - & $a b 46540$ & - & $1 \mu g$ & & & Abcam \\
\hline RNF20 & & $\begin{array}{c}\text { PA5- } \\
19597 \\
\end{array}$ & $1: 1000$ & & & & Thermo Scientific \\
\hline RNF40 & KA7-27 & R9029 & $1: 1000$ & - & & & Sigma \\
\hline SUPT6H & & $\begin{array}{l}\text { A300- } \\
802 A\end{array}$ & $1: 2000$ & $1 \mu g$ & & & Bethyl Lab.Inc. \\
\hline
\end{tabular}




\begin{tabular}{|c|c|c|c|c|c|c|c|}
\hline SUPT6H & & ab-32820 & & & & $1: 200$ & Abcam (for IHC) \\
\hline Vimentin & V-9 & sc-6260 & $1: 1000$ & & & $1: 200$ & Santa Cruz \\
\hline
\end{tabular}

\subsubsection{Secondary Antibodies}

\begin{tabular}{|c|c|c|c|}
\hline Name & Catalog No. & WB Dilution & Source \\
\hline Donkey Anti-Mouse IgG-HRP & $715-036-150$ & $1: 20000$ & $\begin{array}{c}\text { Jackson } \\
\text { ImmunoResearch }\end{array}$ \\
\hline Donkey Anti-Rabbit IgG-HRP & $711-036-152$ & $1: 5000$ & $\begin{array}{c}\text { Jackson } \\
\text { ImmunoResearch }\end{array}$ \\
\hline
\end{tabular}

\subsection{Cells}

\subsubsection{Bacterial Cells}

Escherichia coli DH10BTM Invitrogen $\mathrm{GmbH}$, Karlsruhe

\subsubsection{Human Cell lines}

\begin{tabular}{|c|c|c|c|c|}
\hline Cell Line & Species & Tissue Origin & Disease & Source \\
\hline MCF7 & Human & $\begin{array}{c}\text { Mammary } \\
\text { gland/breast }\end{array}$ & adenocarinoma & $\begin{array}{c}\text { ATCC } \\
\text { (HTB-22) }\end{array}$ \\
\hline MCF10A & Human & $\begin{array}{c}\text { Mammary } \\
\text { gland/breast }\end{array}$ & $\begin{array}{c}\text { nontumorigenic } \\
\text { epithelial cell line }\end{array}$ & $\begin{array}{c}\text { ATCC } \\
\text { (CRL-10317) }\end{array}$ \\
\hline MSC & Human & $\begin{array}{c}\text { Bone marrow } \\
\text { stromal cells }\end{array}$ & $\begin{array}{c}\text { TERT- } \\
\text { immortalized } \\
\text { nontumorigenic } \\
\text { cells }\end{array}$ & $\begin{array}{c}\text { Prof. M. Kassem, } \\
\text { Odense University } \\
\text { Hospital, Denmark }\end{array}$ \\
\hline T47D & Human & $\begin{array}{c}\text { Mammary } \\
\text { gland/breast }\end{array}$ & $\begin{array}{c}\text { ductal carcinoma } \\
\text { ATCC } \\
\text { (HTB-133) }\end{array}$ \\
\hline
\end{tabular}

\subsection{Buffers and Solutions}

\section{RIPA buffer}

$\begin{array}{ll}\text { PBS } & 1 \mathrm{X} \\ \text { NP-40 } & 1 \% \\ \text { Sodium deoxycholate } & 0.5 \% \\ \text { SDS } & 0.1 \%\end{array}$


TBS-T 10X (pH 7.6)

Tris

$0.1 \mathrm{M}$

$\mathrm{NaCl}$

$1.5 \mathrm{M}$

Tween-20

$0.5 \%$

Western salts 10X

Tris

$0.25 \mathrm{M}$

Glycine

$0.86 \mathrm{M}$

SDS

$0.7 \mathrm{mM}$

\section{PBS-T 10X (pH 7.4)}

$\mathrm{NaCl}$

$0.73 \mathrm{M}$

$\mathrm{KCl}$

$0.027 \mathrm{M}$

$\mathrm{NaH}_{2} \mathrm{PO}_{4}{ }^{*} 7 \mathrm{H}_{2} \mathrm{O}$

$14.3 \mathrm{mM}$

$\mathrm{KH}_{2} \mathrm{PO}_{4}$

$14.7 \mathrm{mM}$

Tween-20

$1 \%$

\section{PBS for cell culture}

1 PBS tablet per $500 \mathrm{ml}$ distilled $\mathrm{H}_{2} \mathrm{O}$

\section{PBS-T}

PBS including $0.1 \%(\mathrm{w} / \mathrm{v})$ Tween-20

\section{PCR-Mix 10X}

Tris- $\mathrm{HCl}(\mathrm{pH} 8.8)$

$750 \mathrm{mM}$

$\left(\mathrm{NH}_{4}\right)_{2} \mathrm{SO}_{4}$

$200 \mathrm{mM}$

Tween-20

$0.1 \%$

\section{RT-PCR Master Mix}

PCR-Mix

$\mathrm{MgCl}_{2}$

SYBR Green

dNTPs

Taq-polymerase

Triton X-100

Trehalose
$1 \mathrm{X}$

$3 \mathrm{mM}$

$1: 80000$

$0.2 \mathrm{mM}$

$20 \mathrm{U} / \mathrm{ml}$

$0.25 \%$

$300 \mathrm{mM}$

\section{Blocking solution}

TBST

$1 \mathrm{X}$

Milk

$5 \%$ 
Cell culture freezing medium

DMEM

FBS

DMSO

\section{Chelex (10\%)}

Chelex

\section{Nuclear preparation buffer}

$\mathrm{NaCl}$

EDTA pH 8.0

Tris- $\mathrm{HCl} \mathrm{pH} 7.5$

NP-40

Triton X-100

$\mathrm{NaF}$

\section{Sonication buffer-1}

Tris- $\mathrm{HCl} \mathrm{pH} 8.0$

EDTA

SDS

\section{Sonication buffer-2}

EDTA

Tris-HCl pH 8.0

$\mathrm{NaCl}$

$\mathrm{NP}-40$

$\mathrm{NaF}$

\section{Dilution buffer}

EDTA

Tris-HCl pH 8.0

$\mathrm{NaCl}$

NP-40

$\mathrm{NaF}$

Sodium deoxycholate

\section{IP Buffer}

EDTA
Tris- $\mathrm{HCl} \mathrm{pH} 8.0$
$\mathrm{NaCl}$
$\mathrm{NP}-40$

$50 \mathrm{mM}$

$42 \%$

$50 \%$

$8 \%$

$10 \%(w / v)$ in $\mathrm{H} 2 \mathrm{O}$

$150 \mathrm{mM}$
$20 \mathrm{mM}$
$50 \mathrm{mM}$
$0.5 \%$
$1 \%$
$20 \mathrm{mM}$

$10 \mathrm{mM}$

$1 \%(w / v)$

$20 \mathrm{mM}$

$50 \mathrm{mM}$

$150 \mathrm{mM}$

$1 \%(\mathrm{v} / \mathrm{v})$

$20 \mathrm{mM}$

$20 \mathrm{mM}$

$50 \mathrm{mM}$

$150 \mathrm{mM}$

$1 \%(\mathrm{v} / \mathrm{v})$

$20 \mathrm{mM}$

$0.5 \%(w / v)$

$20 \mathrm{mM}$

$50 \mathrm{mM}$

$150 \mathrm{mM}$

$1 \%(\mathrm{v} / \mathrm{v})$ 
Sodium deoxycholate

\section{Wash buffer}

$\mathrm{LiCl}$

\section{$0.5 \mathrm{M}$}

$\mathrm{NP}-40$

$1 \%(\mathrm{v} / \mathrm{v})$

Sodium deoxycholate

EDTA

$20 \mathrm{mM}$

Tris- $\mathrm{HCl} \mathrm{pH} 8.5$

$10 \mathrm{mM}$

$\mathrm{NaF}$

$20 \mathrm{mM}$

\section{TE buffer}

Tris- $\mathrm{HCl} \mathrm{pH} 8.0$

EDTA

$1 \mathrm{mM}$

\section{Protease, phosphatase and deubiquitinase inhibitors}

Pefabloc

Aprotinin/Leupeptin

BGP

NEM

IAA

$\mathrm{NiCl}_{2}$
$1 \mathrm{mM}$

$1 \mathrm{ng} / \mu \mathrm{l}$

$10 \mathrm{mM}$

$1 \mathrm{mM}$

$10 \mu \mathrm{M}$

$1 \mathrm{mM}$

DMEM cell culture "normal" medium

Phenol red-free high-glucose DMEM

FBS

Penicillin

Streptomycin

Sodium pyruvate
$10 \%$

$100 \mathrm{U} / \mathrm{ml}$

$100 \mu \mathrm{g} / \mathrm{ml}$

$1 \mathrm{mM}$

\section{DMEM/F12 cell culture medium}

Phenol red-free high-glucose DMEM/F12

Horse serum

$5 \%$

EGF

$100 \mu \mathrm{g} / \mathrm{ml}$

Hydrocortisone

$1 \mathrm{mg} / \mathrm{ml}$

Cholera toxin

$1 \mathrm{mg} / \mathrm{ml}$

Insulin

$10 \mathrm{mg} / \mathrm{ml}$

Penicillin

$100 \mathrm{U} / \mathrm{ml}$

Streptomycin

$100 \mu \mathrm{g} / \mathrm{ml}$ 


\section{$\underline{6 x \text { Laemmli buffer }}$}

Tris (pH 6.8)

$0.35 \mathrm{M}$

Glycerol

$30 \%$

SDS

$10 \%$

DTT

$9.3 \%$

Bromophenol blue

$0.02 \%$

SDS separating gel ( $\mathrm{X} \%$ )

Acrylamide

$\mathrm{X} \%$

Tris- $\mathrm{HCl}(\mathrm{pH} 8.8)$

$375 \mathrm{mM}$

SDS

$0.1 \%$

APS

$0.1 \%$

TEMED

$0.04 \%$

\section{SDS stacking gel (5\%)}

Acrylamide

Tris-HCl (pH 6.8)

SDS

APS

TEMED
$5 \%$

$125.5 \mathrm{mM}$

$0.1 \%$

$0.1 \%$

$0.1 \%$

\section{TAE buffer (50x)}

Tris

Acetic acid

$2 \mathrm{M}$

EDTA

$1 \mathrm{M}$

$0.1 \mathrm{M}$

$\underline{\text { Transfer buffer }}$

10x Western salts

$10 \%$

Methanol

$15 \%$

\section{Co-IP Lysis Buffer}

Tris- $\mathrm{HCl}(\mathrm{pH}-7.1)$

$5 \mathrm{mM}$

$\mathrm{NaCl}$

$25 \mathrm{mM}$

Triton X - 100

$0.5 \%$

$\mathrm{NaF}$

$25 \mathrm{mM}$

$\mathrm{Na}_{3} \mathrm{VO}_{4}$

$0.5 \mathrm{mM}$

DTT

$0.2 \mathrm{mM}$ 


\section{Ascorbic acid stock solution (1000x)}

$0.2 \mathrm{M}$ ascorbic acid in sterile water

\section{Calcitriol stock solution (1000x)}

$10 \mu \mathrm{M}$ calcitriol in 100\% DMSO

Dexamethasone stock solution (1000x)

$100 \mu \mathrm{M}$ dexamethasone in $100 \% \mathrm{EtOH}$

\section{ß-glycerol phosphate (BGP) stock solution (100x)}

$1 \mathrm{M}$ BGP in sterile water

\section{IBMX stock solution (100X)}

$0.45 \mathrm{M}$ isobutyl-methyl-xanthine in $100 \% \mathrm{EtOH}$

\section{Troglitazone stock solution (1000x)}

$10 \mathrm{mM}$ Troglitazone in 100\% DMSO

\section{MEM cell culture "normal" medium}

Phenol red-free high-glucose MEM

BGS

Antibiotic-Antimycotic solution

\section{MEM cell culture "adipocyte" medium}

Phenol red-free high-glucose MEM

BGS

Antibiotic-Antimycotic solution

Dexamethasone

IBMX

Insulin

Troglitazone
$15 \%$

$1 \mathrm{X}$

$10 \mathrm{nM}$

$0.45 \mathrm{mM}$

$2 \mu \mathrm{M}$

$10 \mu \mathrm{M}$

\section{MEM cell culture "osteoblast" medium}

Phenol red-free high-glucose MEM

BGS

Antibiotic-Antimycotic solution

Dexamethasone

$\beta$-glycerol phosphate (BGP)

Ascorbic acid

Calcitriol
$10 \%$

$1 \mathrm{X}$

$10 \mathrm{nM}$

$10 \mathrm{mM}$

$0.2 \mathrm{mM}$

$10 \mathrm{nM}$ 


\section{9 Software}

\begin{tabular}{|l|l|}
\hline Name & Source \\
\hline DAVID analysis & http://david.abcc.ncifcrf.gov \\
\hline $\begin{array}{l}\text { Differential gene expression analysis } \\
\text { (DESeq) package }\end{array}$ & $\begin{array}{l}\text { http://www.bioconductor.org/packages/rele } \\
\text { ase/bioc/html/DESeq.html }\end{array}$ \\
\hline Galaxy cistrome & http://cistrome.org/ap/root \\
\hline Galaxy & http://galaxyproject.org/ \\
\hline Gene Set Enrichment Analysis (GSEA) & $\begin{array}{l}\text { Broad Institute, } \\
\text { http://www.broadinstitute.org/gsea/index.jsp }\end{array}$ \\
\hline Image J & http://rsbweb.nih.gov/ij/ \\
\hline AxioVision Software & Carl Zeiss Microlmaging GmbH, Göttingen \\
\hline Primer designing tool NCBI/Primer-BLAST & www.ncbi.nlm.nih.gov/tools/primer-blast/ \\
\hline R statistical software & http://www.r-project.org/ \\
\hline
\end{tabular}




\section{Methods}

\subsection{Cell culture}

\subsubsection{Culturing of Cells}

MCF7 (human breast adenocarcinoma) cells were cultured in phenol red-free highglucose Dulbecco's modified Eagles medium (DMEM) supplemented with 10\% fetal bovine serum (FBS), 100 units $/ \mathrm{ml}$ penicillin, $100 \mu \mathrm{g} / \mathrm{ml}$ streptomycin and $1 \mathrm{mM}$ sodium pyruvate at $37^{\circ} \mathrm{C}$ under $5 \% \mathrm{CO}_{2}$ atmosphere. MCF10A (human mammary epithelial) cells were cultured in DMEM/F12 medium supplemented with $5 \%$ horse serum, $100 \mu \mathrm{g} / \mathrm{ml}$ EGF, $1 \mathrm{mg} / \mathrm{ml}$ hydrocortisone, $1 \mathrm{mg} / \mathrm{ml}$ Cholera toxin, $10 \mathrm{mg} / \mathrm{ml}$ Insulin, 100 units $/ \mathrm{ml}$ penicillin and $100 \mu \mathrm{g} / \mathrm{ml}$ streptomycin under the same conditions. hMSC-Tert20 cells (Simonsen et al., 2002) were cultured in low glucose, phenol red-free MEM supplemented with 10\% FBS and 1X antibiotic antimycotic. T47D (human mammary ductal carcinoma) cells were maintained in RPMI 1640 medium supplemented with $10 \%$ FBS, 100 units $/ \mathrm{ml}$ penicillin, $100 \mu \mathrm{g} / \mathrm{ml}$ streptomycin and $1 \mathrm{mM}$ sodium pyruvate.

\subsection{2 siRNA transfection protocol in 6-well plate}

Reverse-siRNA transfections were performed using LipofectamineTM RNAiMAX according to the manufacturer's instructions. For transfection, $30 \mathrm{pmol}$ of the respective siRNA's were diluted in $500 \mu \mathrm{l}$ of Opti-MEM medium in a well of a 6 -well plate. $5 \mu$ LipofectamineTM RNAiMAX was added to each well and incubated for 20 min at RT. In the meantime, MCF7 or MCF10A cells were trypsinized and diluted in medium without antibiotics. Cells were then counted using Neubauer counting chamber and nearly 250,000 cells (MCF10A) or 300,000 cells (MCF7) from the diluted cells were added to each well already containing the siRNA-LipofectamineTM 
RNAiMAX complexes. The medium was replaced with medium containing antibiotics after $24 \mathrm{~h}$. Cells were harvested in $48 \mathrm{~h}$ or $72 \mathrm{~h}$ after transfection.

\subsubsection{Migration assay}

Migration potential of cells upon various knockdowns or inhibitor treatments was assayed by seeding 50,000 MCF10A cells $48 \mathrm{~h}$ after transfection with the respective siRNAs into $8.0 \mu \mathrm{m}$ PET track-etched membrane cell culture inserts. The inserts were pre-equilibrated for at least $30 \mathrm{~min}$ with serum free medium. After preequilibration, the medium was substituted by normal MCF10A cell culture medium and cells were allowed to migrate through the inserts. Cells were grown for another $48 \mathrm{~h}$ and scraped gently from the upper layer of the inserts using a Q-Tip before fixation with $100 \%$ methanol for 10 min. Migrated cells were stained by crystal violet staining $(0.1 \%(\mathrm{w} / \mathrm{v})$ crystal violet, $10 \%(\mathrm{v} / \mathrm{v})$ formaldehyde) for $10 \mathrm{~min}$. The inserts were rinsed twice in distilled water to remove excess staining. The membrane of the inserts was then visualized under the microscope.

\subsubsection{Mammosphere formation assay}

Single cells were allowed to grow in non-adherent and non-differentiating conditions to determine their in vitro proliferative capacity. Single cell suspensions from MCF10A cells $48 \mathrm{~h}$ after transfection with respective siRNAs were seeded at a density of 10,000 cells per $\mathrm{ml}$ in DMEM/F12 medium supplemented with $2 \%$ B27 (serum free supplement), $5 \mathrm{mg} / \mathrm{ml}$ insulin, $0.5 \mathrm{mg} / \mathrm{ml}$ hydrocortisone, $20 \mathrm{ng} / \mathrm{ml}$ basic fibroblast growth factor (bFGF) and $20 \mathrm{ng} / \mathrm{ml}$ epidermal growth factor (EGF). Cells were grown in 6 well plates coated with 1.2\% polyhema for 6 days and mammospheres formed were counted and images were taken using Zeiss Axiovision software. 


\subsection{Molecular Biology}

\subsubsection{RNA isolation}

RNA isolation from the cultured cells was performed with QIAzol® reagent according to the manufacturer's instructions. Cells were washed twice with PBS, lysed by addition of $500 \mu \mathrm{l}$ of $\mathrm{QIAzo|}{ }^{\circledR}$ reagent to each well (6-well format) and scraped into $1.5 \mathrm{ml}$ tubes. $100 \mu \mathrm{l}$ of chloroform was added to the samples, vortexed for $20 \mathrm{sec}$ and then centrifuged at $10,000 \mathrm{~g}$ for $20 \mathrm{~min}\left(4^{\circ} \mathrm{C}\right)$. The aqueous phase was collected into a fresh $1.5 \mathrm{ml}$ tube and chloroform extraction was performed again followed by 2 $\mathrm{h}$ or overnight isopropanol precipitation at $-20^{\circ} \mathrm{C}$. After that, samples were centrifuged at maximal speed of $12,000 \mathrm{~g}$ for $30 \mathrm{~min}\left(4^{\circ} \mathrm{C}\right)$, pellets were washed twice with $70 \%$ ethanol, dried on vacuum concentrator and re-dissolved in $40 \mu \mathrm{l}$ of DEPC water. RNA concentration was measured using a NanoDrop.

\subsection{2 cDNA synthesis}

For DNA synthesis $1 \mu \mathrm{g}$ of total RNA was mixed with $2 \mu \mathrm{l}$ of $15 \mu \mathrm{M}$ random primers and $4 \mu \mathrm{l}$ of $2.5 \mathrm{mM} \mathrm{dNTP}$ mix and incubated $5 \mathrm{~min}$ at $70^{\circ} \mathrm{C}$. After that $4 \mu \mathrm{l}$ of reverse transcription master mix containing $2 \mu$ 10x reaction buffer, 10 units of RNAse Inhibitor, 25 units of reverse transcriptase and $1.625 \mu$ l of DEPC water were added to each sample. cDNA synthesis was performed at $42^{\circ} \mathrm{C}$ for $1 \mathrm{~h}$ followed by enzyme inactivation for $5 \mathrm{~min}$ at $95^{\circ} \mathrm{C}$. Finally, samples were brought to $50 \mu \mathrm{l}$ volume by DEPC water.

\subsubsection{Quantitative real-time PCR}

One $\mu$ of ChIP or cDNA sample was used for subsequent quantitative real-time PCR analysis with a final reaction volume of $25 \mu$ l. A PCR reaction was setup as follows: $75 \mathrm{mM}$ Tris- $\mathrm{HCl}$ (pH 8.8), $20 \mathrm{mM}\left(\mathrm{NH}_{4}\right)_{2} \mathrm{SO}_{4}, 0.01 \%$ Tween-20, $3 \mathrm{mM} \mathrm{MgCl}, 200 \mu \mathrm{M}$ 
dNTPs, 0.5 U/reaction Taq DNA Polymerase, $0.25 \%$ Triton X-100, 1:80,000 SYBR Green I, $300 \mathrm{mM}$ Trehalose and $30 \mathrm{nM}$ primers.

A two-step PCR protocol was used for each primer pair:

$2 \min -95^{\circ} \mathrm{C}$

$\left[\begin{array}{c}15 \mathrm{sec}-95^{\circ} \mathrm{C} \\ 1 \min -60^{\circ} \mathrm{C}\end{array}\right] \times 40$

The PCR reaction was followed by a melting curve analysis from $60^{\circ} \mathrm{C}$ to $95^{\circ} \mathrm{C}$ with read every $0.5^{\circ} \mathrm{C}$.

cDNA samples were quantified using a standard curve made from all cDNA samples. Prior to statistical analysis all qRT-PCR samples were normalized to $18 \mathrm{~S}$ ribosomal RNA or HNRNPK as an internal reference gene. The expression levels were determined relative to the vehicle treated control sample and expressed as "relative mRNA expression".

ChIP and ChIP input samples were also quantified using a standard curve made from ChIP input DNA. ChIP samples were normalized to their corresponding input samples and expressed as "\% input".

\subsubsection{RNA-sequencing}

RNA-seq library preparation as well as sequencing was carried out at the Transcriptome Analysis Laboratory (TAL), University of Göttingen.

\section{Library Preparation}

Library preparation for RNA-seq was performed using the TruSeq RNA Sample Preparation Kit (Illumina, Cat.No. RS-122-2002) starting from 500 ng of total RNA. 
Accurate quantitation of cDNA libraries was performed by using the QuantiFluor ${ }^{\mathrm{TM}}$ dsDNA System (Promega). The size range of final cDNA libraries was determined by applying the DNA 1000 chip on the Bioanalyzer 2100 from Agilent (280 bp). cDNA libraries were amplified and sequenced by using the cBot and HiSeq2000 from Illumina (SR; 1 x50 bp; 6 GB ca. 30-35 million reads per sample).

Sequence images were transformed with Illumina software BaseCaller to bcl files, which were demultiplexed to fastq files with CASAVA v1.8.2. Quality check was done via fastqc (v. 0.10.0, Babraham Bioinformatics).

\section{Data Analysis}

The analysis involves detection of differentially expressed genes in various conditions. The raw data Fastq files from the sequencer were analyzed using Bowtie 2.0 on Galaxy and DESeq package in R-script. Bowtie 2.0 is a short read aligner which functions to align short DNA sequences (as reads from sequencer) to the human genome (transcriptome) - hg19 mRNA. The output provides RPKM values that signify number of reads per kilobase of DNA per million mapped reads for each gene, for each sample. This output file was used for DESeq package in R-script (Anders and Huber, 2010). Heatmaps were generated to visualize the differential expression of genes in various knockdowns. The list of differentially regulated genes was used for Gene Set Enrichment Analysis (GSEA) as well as DAVID (Gene ontology). GSEA is a computational method for determining statistically significant differences between two phenotypes. DAVID is a web-based program for analyzing the list of genes to their associated biological annotation. 


\subsubsection{Chromatin immunoprecipitation (ChIP)}

Protein-DNA complexes in cells grown in $10 \mathrm{~cm}$ plates were crosslinked for $10 \mathrm{~min}$ by adding $1 \%$ formaldehyde in PBS. Glycine to a final concentration of $125 \mathrm{mM}$ was added for $5 \mathrm{~min}$ in order to quench the formaldehyde. Cells were then washed twice with ice-cold PBS and scraped in $1 \mathrm{ml}$ of Nuclear preparation buffer containing inhibitor cocktail. All further steps were performed on ice or at $4^{\circ} \mathrm{C}$. Nuclear pellet was isolated from the lysate by centrifugation at $12,000 \mathrm{~g}$ for $1 \mathrm{~min}$ and pellet was washed again with $1 \mathrm{ml}$ of Nuclear preparation buffer. Finally, the nuclear pellet was resuspended by gentle pipetting in $150 \mu \mathrm{l}$ Sonication buffer-1 (with $1 \%$ SDS) containing inhibitor cocktail and incubated at $4^{\circ} \mathrm{C}$ on a wheel for $15 \mathrm{~min}$. The SDS content was diluted to $0.5 \%$ SDS using $150 \mu \mathrm{l}$ Sonication buffer-2 (no SDS). The sonication process was done in a Bioruptor for $30 \mathrm{~min}$ with $30 \mathrm{sec}$ on/off cycles. The soluble chromatin was then cleared from the debris by centrifugation at $12000 \mathrm{~g}$ for 10 min $\left(8^{\circ} \mathrm{C}\right)$ and pre-cleared with $100 \mu \mathrm{l}$ of $50 \%$ slurry of Sepharose beads for $1 \mathrm{~h}$. After the pre-clearing the chromatin was centrifuged, diluted in Dilution buffer, aliquoted, frozen in liquid nitrogen and stored at $-80^{\circ} \mathrm{C}$. For the immunoprecipitation, $100 \mu \mathrm{l}$ of chromatin extract was diluted up to $500 \mu \mathrm{l}$ with IP buffer (containing inhibitor cocktail) and incubated overnight with the indicated amount of antibodies. Immunoglobulin bound complexes were precipitated by adding $30 \mu \mathrm{l}$ of $50 \%$ slurry of Protein-A or Protein-G Sepharose and incubated for 2 h. Following incubation, the samples were centrifuged at $2,000 \mathrm{~g}$ for $2 \mathrm{~min}$. The beads were washed with series of buffers as follows: ice-cold IP buffer twice, wash buffer thrice, IP buffer twice and TE buffer twice. The crosslink was reversed by adding $10 \%$ slurry Chelex with subsequent heating to $95^{\circ} \mathrm{C}$ for $10 \mathrm{~min}$. The proteins in the samples were eliminated by adding $20 \mu \mathrm{g}$ of Proteinase $\mathrm{K}$ and incubating for $30 \mathrm{~min}$ at $55^{\circ} \mathrm{C}(800 \mathrm{rpm})$. 
Proteinase $\mathrm{K}$ was inactivated by heating at $95^{\circ} \mathrm{C}$ for $10 \mathrm{~min}$. The samples were centrifuged at $12,000 \mathrm{~g}$ for $1 \mathrm{~min}\left(4^{\circ} \mathrm{C}\right)$ and the supernatant was analyzed by quantitative real-time PCR. The background binding was determined by performing a ChIP with a non-specific IgG antibody. ChIP inputs preparation: $10 \mu \mathrm{l}(10 \%$ relative to ChIPs) of chromatin extracts were incubated overnight at $-20^{\circ} \mathrm{C}$. The inputs were processed with Chelex addition as described above for ChIP samples. ChIP samples were normalized to input DNA samples, and displayed as "\% input".

\subsubsection{Chromatin immunoprecipitation-sequencing (ChIP-seq)}

ChIP-sequencing involves chromatin immunoprecipitation followed by DNA sequencing in order to determine the sites for protein-DNA interactions in the genome. The chromatin for ChIP-seq was prepared as mentioned above. For sequencing, the DNA was isolated using phenol chloroform extraction.

DNA isolation using phenol chloroform extraction : For this purpose, $50 \mu \mathrm{l}$ of $10 \mathrm{mM}$ Tris $\mathrm{HCl} \mathrm{pH} 8.0$ containing $10 \mu \mathrm{g}$ of RNAse A was added to the already washed chromatin-bound beads as well as the input samples and incubated for $30 \mathrm{~min}$ at $37^{\circ} \mathrm{C}$. Then, $50 \mu \mathrm{l}$ of $2 x$ Sonication buffer -2 and $20 \mu \mathrm{g}$ of Proteinase $\mathrm{K}$ were added and the samples were incubated overnight at $65^{\circ} \mathrm{C}$ with a subsequent centrifugation step at 2,000 g for 2 min at RT. The supernatant was collected, and the beads were rinsed again with $100 \mu \mathrm{l}$ of $10 \mathrm{mM}$ Tris $\mathrm{pH}$ 8.0. The samples were centrifuged and the supernatant was added to the first one. For extraction, $10 \mu \mathrm{l}$ of $8 \mathrm{M} \mathrm{LiCl}, 4 \mu \mathrm{l} \mathrm{co-}$ precipitant (linear polyarcylamide) and $200 \mu \mathrm{l}$ phenol/chloroform/isoamyl alcohol (25:24:1) were added, samples were vortexed for $30 \mathrm{sec}$ and centrifuged for $2 \mathrm{~min}$ at full speed. The aqueous phase was collected and the phenol phase was back extracted with $200 \mu \mathrm{l} 10 \mathrm{mM}$ Tris $\mathrm{HCl} \mathrm{pH} 8.0$ and $400 \mathrm{mM} \mathrm{LiCl}$. After vortexing and centrifugation, the second aqueous phase was pooled with the first one and 
precipitation was performed by addition of $100 \% \mathrm{EtOH}$ and incubation for $2 \mathrm{~h}$ at $80^{\circ} \mathrm{C}$. After that, samples were centrifuged at maximal speed for $30 \mathrm{~min}\left(4^{\circ} \mathrm{C}\right)$, pellets were washed with $70 \%$ ethanol, dried on vacuum concentrator and redissolved in $40 \mu$ l of water.

$5 \mu \mathrm{l}$ of the DNA was used for qRT-PCR to confirm the efficient chromatin immunoprecipitation and $35 \mu \mathrm{l}$ for ChIP-seq. DNA concentration was measured using a Qubit dsDNA HS assay on a Qubit® 2.0 Fluorometer.

\section{Shearing check}

The efficiency of sonication was determined by performing a shearing check. Briefly, $10 \mu \mathrm{l}$ of sheared chromatin was used for phenol chloroform extraction. After resuspension of the DNA in $15 \mu \mathrm{l}$ of $10 \mathrm{mM}$ Tris $\mathrm{HCl} \mathrm{pH} 8.0,100 \mu \mathrm{g} / \mathrm{ml}$ RNAse A was added and the mixture was incubated for $1 \mathrm{~h}$ at $37^{\circ} \mathrm{C}(700 \mathrm{rpm})$. The DNA was then run on a $1.5 \%$ agarose gel and analyzed on the gel documentation. Efficient shearing consisted of a smear from about 150 to $1 \mathrm{~kb}$ with a maximum around 200$400 \mathrm{bp}$.

\section{Library preparation}

ChIP-seq library preparation was performed using NEBNext Ultra DNA library prep kit for Illumina (E7370) as per manual's instructions. 5 - 50 ng of fragmented DNA from ChIP was resonicated for $15 \mathrm{~min}$ in Bioruptor to ensure small fragments suitable for sequencing. End preparation was performed by adding end prep enzyme mix and end repair reaction buffer $(10 X)$ to a final reaction volume of $65 \mu$ l. Samples were placed on a thermocycler with cycles of $20^{\circ} \mathrm{C}$ for 30 min and $65^{\circ} \mathrm{C}$ for 30 min followed by adaptor ligation with blunt/TA ligase master mix, NEBNext adaptor and 
ligation enhancer to a final volume of $83.5 \mu$. Samples were incubated at $20^{\circ} \mathrm{C}$ for 15 min, then $3 \mu$ of User enzyme was added and placed at $37^{\circ} \mathrm{C}$ for $15 \mathrm{~min}$. Adaptor ligated DNA was cleaned up using 0.9x AMPure XP beads on magnetic stand and finally DNA was resuspended in $28 \mu \mathrm{l}$ of $10 \mathrm{mM}$ Tris $\mathrm{pH}$ 8.0. From this, $23 \mu \mathrm{l}$ of DNA was used for PCR amplification followed by clean up of PCR amplified product using AMPure XP beads. The DNA was resuspended in $33 \mu \mathrm{l}$ of $10 \mathrm{mM}$ Tris $\mathrm{pH} 8.0$ and analyzed on Bioanalyzer.

Quantitation of cDNA libraries was done on an Invitrogen Qubit 2.0 Fluorometer and the size range of cDNA libraries was performed on an Agilent Bioanalyzer 2100 (High Sensitivity DNA Assay). cDNA libraries were amplified and sequenced by using the cBot and HiSeq2500 from Illumina (20-25 million reads per sample). Sequence images were transformed with Illumina software BaseCaller to bcl files, which were demultiplexed to fastq files with CASAVA v1.8.2. Quality check was done via fastqc (v. 0.10.1, Babraham Bioinformatics).

\section{Data Analysis}

ChIP-seq analysis was performed by Prof. Steven A. Johnsen. The fastq files from the sequencer or downloaded from ENA database were mapped to the human genome using Bowtie on Galaxy server which involves alignment of short DNA reads to the human genome - hg19. The BAM files from Bowtie were used for Model Based Analysis of ChIP-seq (MACS), which involves the identification of the peaks for the protein binding, commonly referred to as peak calling with peak $p$-values $\leq$ 10e-5. This generated Bed file containing the peak location and Wiggle (Wig) file containing signal profile further used to analyze the genome-wide recruitment of proteins or histone modifications. Cis-regulatory Element Annotation System (CEAS) 
and SitePro packages available on Galaxy were used to determine the sites of recruitment of proteins or histone modifications as well as average signal intensities at various genomic locations.

\subsection{Protein biochemistry}

\subsubsection{SDS-PAGE}

SDS-PAGE is used for separating proteins using denaturing agent SDS (sodium dodecylsulfate) on a polyacrylamide gel upon electrophoresis (Laemmli, 1970). For protein preparation, cells were lysed in RIPA buffer containing $1 \mathrm{mM}$ Pefabloc, 1 $\mathrm{ng} / \mu \mathrm{l}$ Aprotinin/Leupeptin, $10 \mathrm{mM} \mathrm{BGP}$ and $1 \mathrm{mM}$ NEM. Genomic DNA was sheared by sonication where samples were sonicated for $10 \mathrm{sec}$ at $10 \%$ power using a tip sonicator. Before loading, protein samples were boiled in Laemmli Buffer for 10 min and then subjected to SDS-PAGE. The composition of stacking and resolving gel is described in section 2.8. Polyacrylamide gels were run in SDS running buffer at 20 $\mathrm{mA} / \mathrm{gel}$.

\subsubsection{Western blot analysis}

After electrophoresis, proteins were separated according to their molecular weight that are later identified (Towbin et al., 1979) using target protein specific antibodies. Separated proteins were transferred at $100 \mathrm{~V}$ to PVDF membranes using transfer buffer for $90 \mathrm{~min}$, depending on the size of the protein. The membranes were incubated for $1 \mathrm{~h}$ in TBS-T and 5\% (w/v) dry milk to block non-specific antibody binding. Afterwards the membranes were incubated for $1 \mathrm{~h}$ at room temperature or overnight at $4^{\circ} \mathrm{C}$ in the same blocking buffer containing the respective primary antibodies, diluted as described in the antibody table (2.6.3.1). After washing thrice with TBS-T, the membranes were incubated for $1 \mathrm{~h}$ with the corresponding horseradish peroxidase-conjugated anti-mouse $\lg G$, anti-rabbit $\lg G$ or anti-rat $\lg G$ 
secondary antibodies in appropriate dilutions. Further washing thrice with TBS-T, HRP signals were detected using enhanced chemoluminescence and exposed to Xray films.

\subsubsection{Immunohistochemistry on paraffin sections}

For immunostaining, paraffin-embedded sections were de-paraffinized and rehydrated as stated in established procedures. Sections were incubated in xylene for $20 \mathrm{~min}$, followed by rehydration in a 100\%, 90\% and $70 \% \mathrm{EtOH}$ series before washing with PBS. Proteins were then unmasked by boiling slides in $10 \mathrm{mM}$ citric acid/sodium-phosphate. After washing with PBS, sections were quenched for endogenous peroxidase activity with $3 \%$ hydrogen peroxide in PBS for 45 min at RT and then blocked using 5\% FBS diluted in PBS for $1 \mathrm{~h}$ at RT. The primary antibody diluted in PBS containing 5\% FBS was applied and incubated overnight at $4^{\circ} \mathrm{C}$ in a humid chamber. Sections were washed using PBS before adding the biotinylated secondary antibody 1:200 diluted in PBS and incubated for $1 \mathrm{~h}$ at RT. Sections were washed with PBS followed by Avidin-Peroxidase incubation 1:1,000 diluted in PBS for 45 min. Staining signals were detected using diaminobenzidine (DAB) substrate. Hematoxyline (Mayer's hemalaun solution) was used for counterstaining. Histological slides were digitized with a ScanScope XT (Aperio) at 400x magnification. Color intensity quantification was achieved with ImageJ software (Schneider et al., 2012) by manually indicating the nuclei as region-of-interests and performing colour deconvolution (Ruifrok and Johnston, 2001).

\subsubsection{Micrococcal Nuclease Digestion}

MCF7 cells were scraped in hypotonic lysis buffer (10\% sucrose w/v, $10 \%$ glycerol v/v, $10 \mathrm{mM}$ HEPES $\mathrm{pH}$ 7, $10 \mathrm{mM} \mathrm{KCl,} 2 \mathrm{mM} \mathrm{MgCl}$ and $50 \mu \mathrm{g} / \mathrm{ml} \mathrm{BSA)} \mathrm{containing}$ protease inhibitors (1 $\mathrm{ng} / \mu \mathrm{l}$ aprotinin/leupeptin, $1 \mathrm{mM}$ Pefabloc), deubiquitinating 
enzyme inhibitors ( $1 \mathrm{mM}$ N-ethylmaleimide, $10 \mu \mathrm{M}$ iodoacetamide) and $5 \mathrm{mM}$ sodium butyrate, pelleted by centrifugation, and resuspended in lysis buffer with $0.1 \%$ Triton X-100 and incubated $10 \mathrm{~min}$ on ice for permeabilization. The nuclei were washed once with MNase buffer (50 mM Tris $\mathrm{pH} 8,5 \mathrm{mM} \mathrm{CaCl}_{2}$ and $100 \mu \mathrm{g} / \mathrm{ml} \mathrm{BSA}$ ) and resuspended in MNase buffer. MNase $(2,000,000$ gels units/ml, diluted 1:400, New England Biolabs) was added to the nuclei and digestion was stopped after 4 minutes by adding 1 volume Stop solution (50 mM EDTA, 1\% SDS, $100 \mathrm{mM}$ Tris pH 7.5 and $200 \mu \mathrm{g} / \mathrm{ml}$ Proteinase K). Proteinase $\mathrm{K}$ digestion was performed overnight at $50^{\circ} \mathrm{C}$. DNA was purified using phenol/chloroform/isoamyl alcohol extraction in presence of $0.4 \mathrm{M} \mathrm{LiCl}$ and ethanol precipitation with $10 \mu \mathrm{g}$ of linear polyacrylamide as coprecipitant (Bioline). RNA was digested by $200 \mu \mathrm{g} / \mathrm{ml}$ RNase $A$ for $1 \mathrm{~h}$ at $37^{\circ} \mathrm{C}$. DNA concentration was measured using Nanodrop. Digestion patterns were analyzed on $2 \%$ agarose gel and using a Bioanalyzer with the DNA 1000 kit (Agilent).

\subsubsection{Co-immunoprecipitation}

Cells were washed with PBS and scraped in $1.5 \mathrm{ml}$ Co-IP lysis buffer containing protease inhibitors, vortexed for $15 \mathrm{sec}$ and incubated for $1 \mathrm{~h}$ on a rotator at $4^{\circ} \mathrm{C}$. The lysate was sonicated for $30 \mathrm{sec}$ three times and centrifuged at $10,000 \mathrm{rpm}$ for 5 min at $4^{\circ} \mathrm{C}$. Supernatant was transferred to a fresh $1.5 \mathrm{ml}$ tube and resonicated followed by centrifugation. The supernatant was collected and $100 \mu$ was taken as input. Appropriate amount of supernatant was incubated with $2-3 \mu \mathrm{g}$ of antibody for $4 \mathrm{~h}$ or overnight at $4^{\circ} \mathrm{C}$ on a rotator. The next day, $30 \mu \mathrm{l}$ of $50 \%$ slurry of Protein $\mathrm{A}$ or $\mathrm{G}$ sepharose was added and incubated for $2 \mathrm{~h}$ on a rotator at $4^{\circ} \mathrm{C}$. Beads were collected by centrifugation at $1000 \mathrm{rpm}$ for $2 \mathrm{~min}$, washed twice with lysis buffer and 
resuspended in $50-100 \mu$ l of lysis buffer. Beads were boiled in Laemmli buffer, loaded on SDS-PAGE and protein was detected using antibody of interest.

\subsubsection{Immunofluorescence}

Cells grown on chamber slides were washed with PBS and fixed with 4\% paraformaldehyde for $15 \mathrm{~min}$ at RT. Cells were washed twice with PBS and permeabilized using $0.1 \%$ Triton $\mathrm{X}-100$ for 10 min. After washing twice with PBS, cells were blocked with $10 \%$ FBS for 10 min followed by overnight incubation with primary antibody dilution in $10 \%$ FBS. Next day, unbound antibody was washed away by washing twice with PBS and incubated with Alexa-488 or Alexa-594 conjugated secondary antibodies. Cells were washed twice with PBS and stained for DAPI and mounted with coverslips using mounting medium. Images were taken using LSM 510 META confocal microscope and analyzed using the LSM Image Browser.

\subsection{Chemical staining}

\subsubsection{Alkaline phosphatase staining}

Osteoblast differentiation efficiency was determined by staining of alkaline phosphatase activity. Staining was performed with alkaline phosphatase kit for leukocytes according to manufacturer's instructions. All steps were performed at RT. First, cells were washed with PBS and fixed for $30 \mathrm{sec}$ with Citrate fixing solution containing (for $98 \mathrm{ml}$ ): $66 \mathrm{ml}$ acetone, $25 \mathrm{ml}$ Citrate solution and $8 \mathrm{ml} \mathrm{37 \%}$ formaldehyde. After fixation, cells were washed thrice with distilled water and incubated for 15 min with diazonium salt followed by rinsing with distilled water and drying. Diazonium salt preparation: $1 \mathrm{ml}$ of FRV-Alkaline solution was mixed with 1 $\mathrm{ml}$ of sodium nitrate solution and incubated for $2 \mathrm{~min}$. Then $45 \mathrm{ml}$ of distilled water and $1 \mathrm{ml}$ of Naphtol AS-BI Alkaline solution were added to the mix. Diazonium salt 
was prepared freshly before each staining. Pictures of the stained plates were taken under light microscope using 10x magnification.

\subsubsection{Oil Red O staining}

Lipid drops were stained with Oil Red $O$ staining to determine the adipocyte differentiation efficiency. All steps were performed at RT. Oil Red O working solution was prepared by mixing 3 parts of Oil Red O stock solution $(300 \mathrm{mg} / \mathrm{ml}$ of Oil Red O powder in $99 \%$ isopropanol) with 2 parts of distilled water and incubated for 10 min followed by filtration. Cells were washed with PBS, fixed with $10 \%$ formaldehyde for 30 min and incubated with $60 \%$ isopropanol for $5 \mathrm{~min}$. Then, cells were stained with

Oil Red $\mathrm{O}$ working solution for $5 \mathrm{~min}$ followed by rinsing with distilled water and drying. Pictures of the stained plates were taken under light microscope using 10x magnification.

\subsubsection{Carmine alum staining for mammary ducts}

Tissues were fixed overnight in $4 \%$ paraformaldehyde and then rinsed with PBS. Fixed tissues were then pressed between glass slides before staining with carmine alum for 4 hours at room temperature. Carmine alum stain was prepared by mixing $1 \mathrm{~g}$ of carmine and $2.5 \mathrm{~g}$ of aluminum potassium sulfate in $500 \mathrm{ml}$ of distilled water and boiled for $20 \mathrm{~min}$, filtered and refrigerated. The stained tissues were then put in series of $50 \%, 70 \%$ and $100 \%$ ethanol each for $5 \mathrm{~min}$. The tissues were then cleared in xylene overnight. The next day, they were transferred to methyl salicylate until the images were taken.

\subsection{RNF40 conditional knockout mouse model}

Cell culture experiments had shown that RNF40 and H2Bub1 were essential for cellular differentiation, it was then anticipated that Rnf40 null mouse would most 
likely be lethal. Thus, a conditional targeting approach was pursued by designing a construct in which exons 3 and 4 of the mouse Rnf40 gene were surrounded by loxP sites. For selection, the neomycin resistance cassette surrounded by two FRTsequences was included in the construct. This approach facilitates deletion of the targeted gene in a time and tissue-dependent manner. The inclusion of the FRTsites enabled FLP recombinase-mediated excision of the Neomycin-cassette, which could otherwise interfere with RNF40 expression in vivo (Pham et al., 1996); (Ren et al., 2002); (Scacheri et al., 2001). The deletion of the resistance cassette was achieved by crossing the Rnf $40^{\text {loxPNeo }}$ mice to a mouse strain expressing the FLP recombinase under the control of the Rosa26 promoter (Farley et al., 2000). The resulting recombination of the transgene led to a functional Rnf40 gene locus carrying two loxP sites surrounding exons 3 and 4 (Fig. 6A). The effect of Rnf40 deletion on mammary epithelium maintenance was examined by crossing with the MMTV-Cre mice. Cre recombinase was under the control of mouse mammary tumor virus (MMTV) long terminal repeat (LTR) in order to restrict the expression to mammary tissue (Wagner et al., 1997). RNF40 $0^{\text {loxP/wt }}$ mice had been obtained, verified and crossed to the MMTV-Cre mouse strains which were further crossed to RNF40 ${ }^{\text {loxPlloxP. }}$. The female mice having RNF40 $0^{\text {loxPlloxP }}$ and Cre expression were allowed to develop for 6-weeks to determine the role of RNF40 and H2Bub1 in mammary epithelium development and maintenance. (Fig. 6B) 

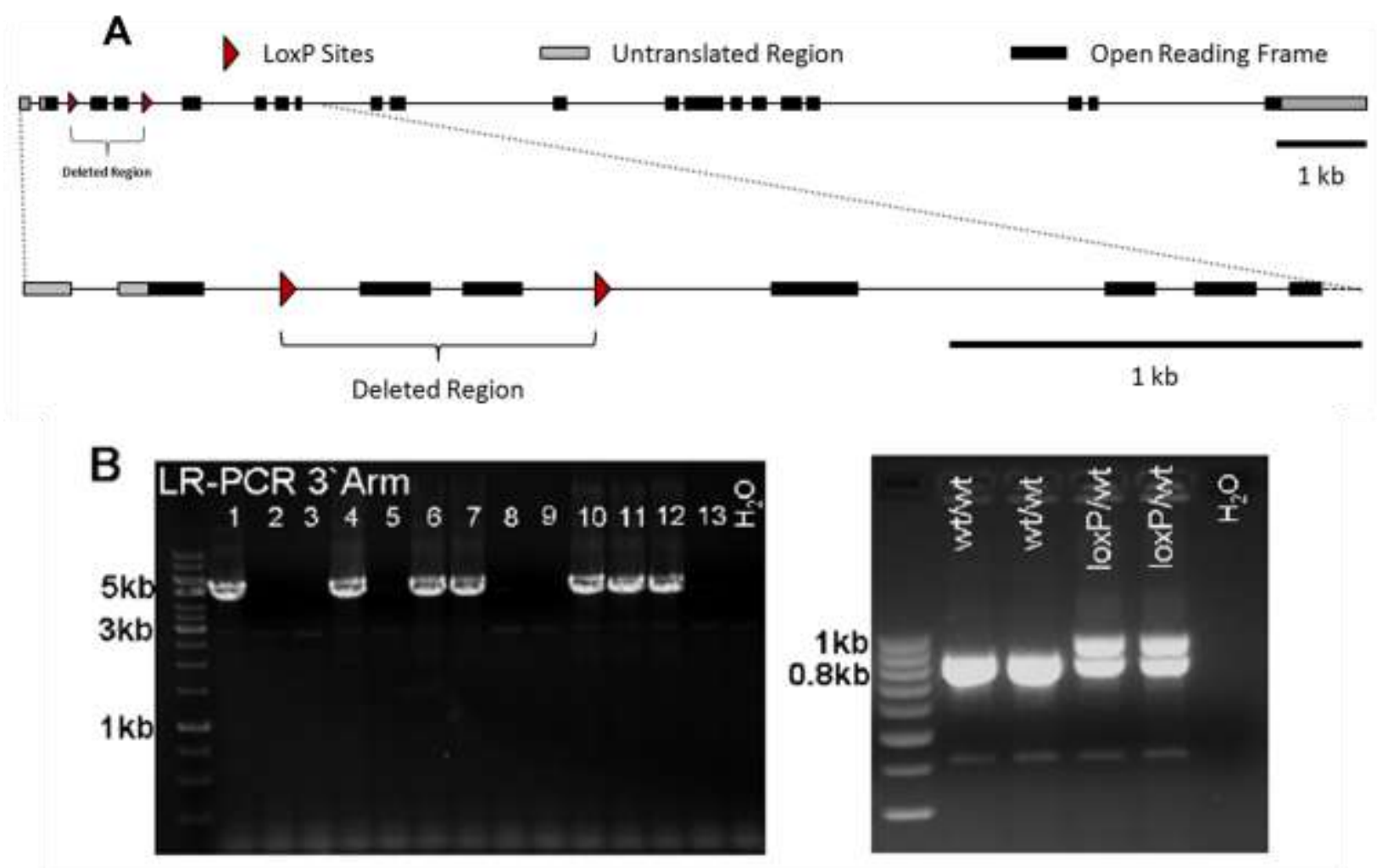

Fig. 6. Detailed graphical overview for the Rnf40 conditional knockout mouse construct and verification. (A) Graphical overview of the Rnf40-construct. (B) Representative picture on the right side of a long-range PCR amplifying a 5382bp fragment of the RNF40 conditional knockout gene using one primer placed within the Neomycin-resistance cassette and the other primer outside of the construct. The amplification of a product with the correct size verifies that the construct has integrated into the correct genomic locus. The right side displays genotyping results using a two primer strategy flanking exon 3 and 4 . This PCR strategy yields an $803 \mathrm{bp}$ product for the wild-type allele while the additional sequences of the loxP sites leads to a 936bp fragment. 


\section{Results}

During development, undifferentiated cells receive extracellular signals and undergo differentiation to form specialized cells. On the contrary, dedifferentiation is considered to be the reversal of the normal development cycle where specialized cells regress back to a stem cell state and has been reported in various cancers. There is a fine balance between these two states and the shift towards one determines the cell fate to undergo differentiation or become dedifferentiated. Our group previously addressed the role of H2Bub1 during cellular differentiation (Karpiuk et al., 2012b). It is now well characterized that H2Bub1 levels increase during differentiation of somatic and pluripotent stem cells (Karpiuk et al. 2012, (Fuchs et al., 2012); Chen et al. 2012). Furthermore, it was found that H2Bub1 plays a tumor suppressor function (Shema et al. 2008, (Prenzel et al., 2011) and could determine tumor malignancy. H2Bub1 levels were found to decrease during tumor progression in breast cancer (Prenzel et al., 2011). Consistently, we wanted to investigate a potential role of H2Bub1 in suppressing a stem cell phenotype in mammary epithelial and breast cancer cells.

\subsection{Estrogen signaling and dedifferentiation}

It is well established that breast cancers having luminal and myoepithelial phenotypes display distinct gene expression patterns which correlate with patient outcome (Abd El-Rehim et al., 2004). While luminal tumors which express ERa and CK19, are generally lower grade and correlate with better overall patient survival, tumors with a myoepithelial phenotype generally have a poorer prognosis and are characterized by the expression of mesenchymal markers like aSMA (ACTA2). Thus, ERa plays a central role in maintaining a luminal epithelial phenotype. Absence of 
ERa leads to a switch from a luminal to a myoepithelial phenotype thereby promoting a more dedifferentiated and aggressive phenotype. It was previously shown that H2Bub1 and RNF40 regulated ERa dependent gene transcription and that H2Bub1 levels in human breast tissue sections decreased with tumor progression (Prenzel et al., 2011). The link between H2Bub1 and P-Ser2 RNA Polymerase II mediated via CDK9-WAC-RNF40 axis was also established (Pirngruber et al., 2009a). We then wanted to investigate the role of histone chaperone, SUPT6H (human homolog of yeast Spt6), known to interact with P-Ser2 RNA Polymerase II through its SH2 domain in estrogen-dependent gene transcription and examine its connection to the H2Bub1 pathway.

\subsubsection{SUPT6H is required for ERa activity}

Given the importance of transcriptional elongation in the regulation of ERa activity, we hypothesized that the histone chaperone and transcriptional elongation regulatory protein, SUPT6H may also be required for estrogen-regulated transcription. In order to test this hypothesis, we compared the effects of siRNAmediated knockdown of SUPT6H to those of the pure ERa antagonist, ICI 182780 on the induction of several estrogen regulated genes in the ERa-positive luminal breast cancer cells, MCF7 and T47D. ICI 182780 is a pure antiestrogen which blocks ERa nucleocytoplasmic shuttling and nuclear uptake, thereby leading to ERa degradation (Dauvois et al., 1993). Treatment of cells with ICI 182780 inhibits ERa dependent gene transcription. As expected, ICI 182780 treatment decreased the induction of the investigated direct ERa target genes (CXCL12, GREB1 and PGR) in both the cell lines (Fig. 7A and B). SUPT6H knockdown also showed similar effects in both the cell lines thereby indicating that it plays an essential role in estrogen-regulated transcription. 
A

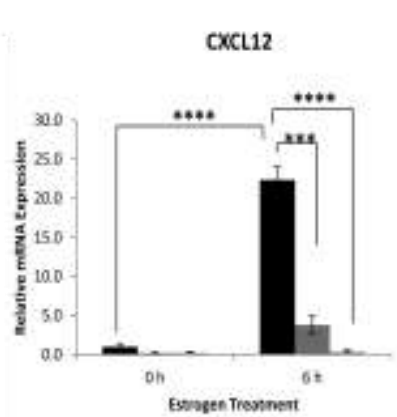

B

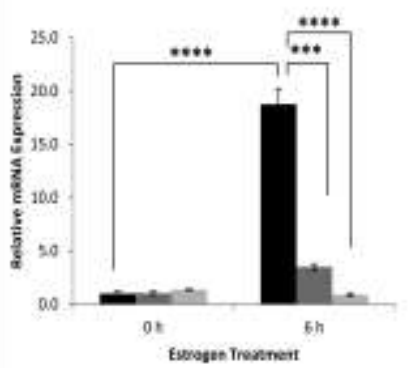

GREB1

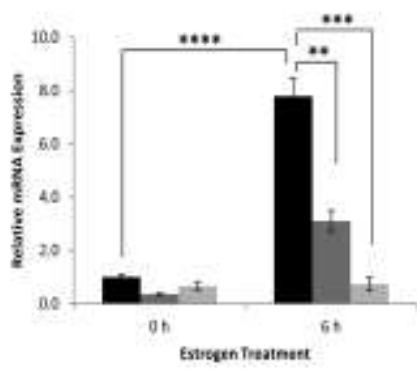

GREB1

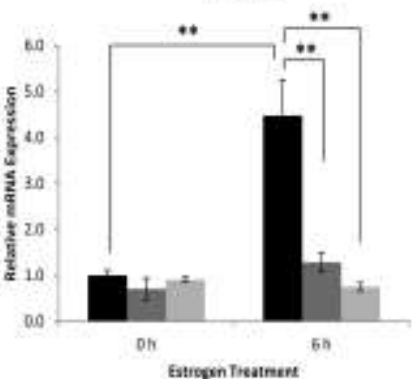

Control
PGR

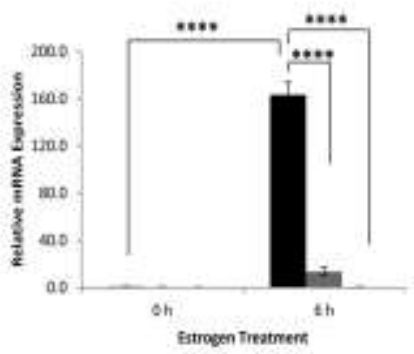

PGR

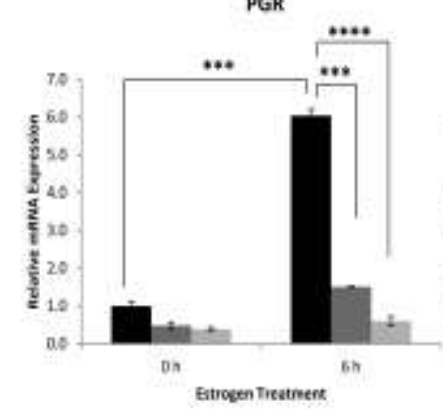

SUPT6H

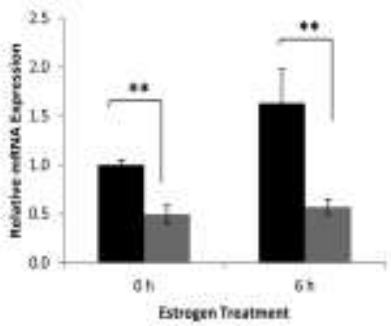

SUPT6H

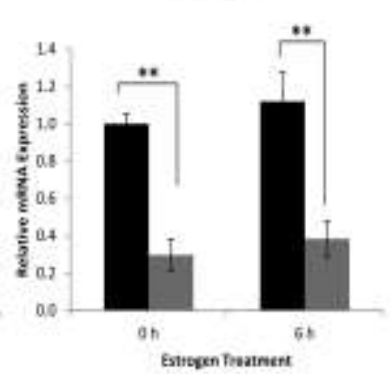

Fig. 7. SUPT6H knockdown decreases estrogen-induced gene expression. (A and B) MCF7 and T47D cells were transfected with either control or SUPT6H siRNAs or treated with ICI 182780, grown for $24 \mathrm{~h}$ before switching to hormone-deprived medium and grown for another $24 \mathrm{~h}$. Cells were then stimulated with $10 \mathrm{nmol} / \mathrm{L} 17 \beta$-estradiol (E2) for $6 \mathrm{~h}$ and the expression levels of $C X C L 12$, GREB1, $P G R$ and SUPT6H were analyzed by qPCR. Gene expression levels were normalized to $18 \mathrm{~S}$ ribosomal RNA, graphed relative to the control sample and expressed as "Relative mRNA Expression"; mean values + s.d., $n=3$. For statistical significance, ANOVA test was performed and indicated by ${ }^{*}(p \leq 0.05),{ }^{* *}(p \leq 0.01),{ }^{* * *}(p \leq 0.001)$ and ${ }^{* * *}(p \leq 0.0001)$.

The effect on estrogen-induced gene expression upon SUPT6H knockdown was further confirmed using single siRNAs for SUPT6H on two representative estrogenregulated genes, CXCL12 and PGR (Fig. 8A). SUPT6H knockdown did not affect the ERa expression as validated by qPCR (Fig. 8B) and western blot (Fig. 8C).

Furthermore, since SUPT6H displays histone chaperone activity and may therefore promote the opening of chromatin and binding of ERa, we next tested whether its depletion affects ERa recruitment to chromatin at Estrogen Response Elements (ERE) on two well characterized estrogen-responsive genes (GREB1 and PGR). 
A
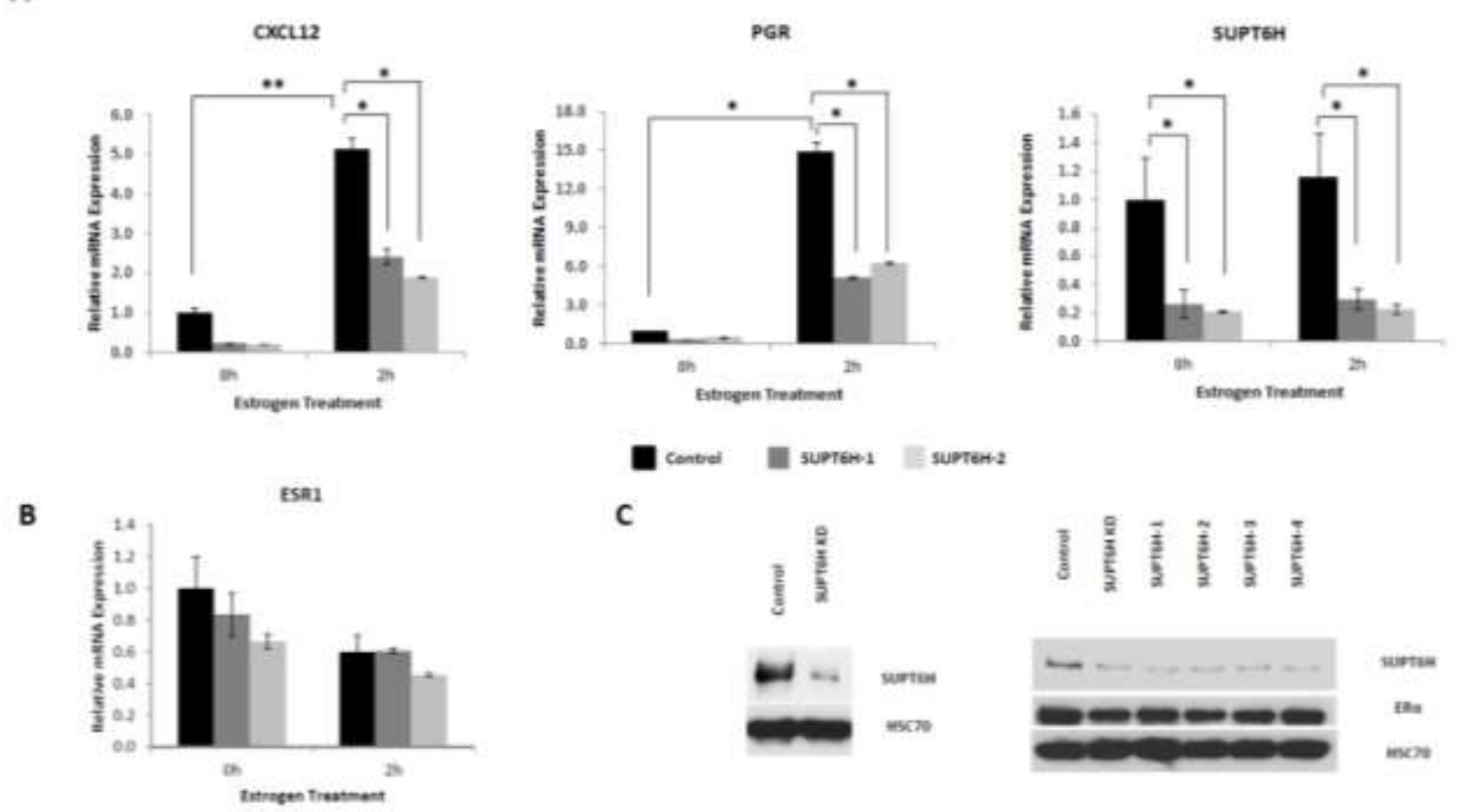

Contiel If supren:1 suprom:

c
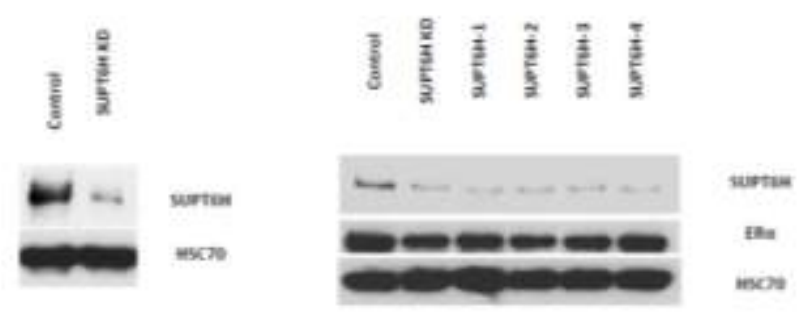

Fig. 8. Effect of single siRNA SUPT6H knockdown on estrogen-induced gene expression. MCF7 cells transfected with control or single siRNAs for SUPT6H were analyzed by qPCR (A and B) and Western blot (C). ESR1 mRNA levels (B) as well as ERa protein levels (C) were not affected with SUPT6H knockdown. HSC70 is shown as a loading control. For RNA experiment, MCF7 cells transfected with control or SUPT6H siRNAs, were grown for $8 \mathrm{~h}$ and then switched to hormone deprived medium for $14 \mathrm{~h}$. Cells were then stimulated with $10 \mathrm{nmol} / \mathrm{L} 17 \beta$-estradiol (E2) for $2 \mathrm{~h}$ and expression levels were analyzed. Gene expression levels were normalized to $18 \mathrm{~S}$ ribosomal mRNA, graphed relative to the control sample and expressed as "Relative mRNA Expression"; mean values + s.d., $n=3$. For protein samples, MCF7 cells transfected with control or SUPT6H siRNA pool or single SUPT6H siRNAs were grown for $24 \mathrm{~h}$ and then harvested. For statistical significance, ANOVA test was performed and indicated by * $(p \leq 0.05)$ and ${ }^{* *}(p \leq 0.01)$.

Indeed, chromatin immunoprecipitation (ChIP) analysis revealed less ERa recruitment to previously identified distal ERa binding sites (Carroll et al., 2006) of the estrogen regulated genes GREB1 (5 kb upstream relative to TSS) and PGR (12 kb downstream relative to TSS) following SUPT6H knockdown (Fig. 9A). Importantly, SUPT6H was recruited both to the distal EREs as well as to the 3' ends (Fig. 9B and C) of these genes and its recruitment was decreased following SUPT6H knockdown. This supports a role for SUPT6H in regulating estrogen-dependent gene expression at least in part via regulation of ERa recruitment to EREs. 
A
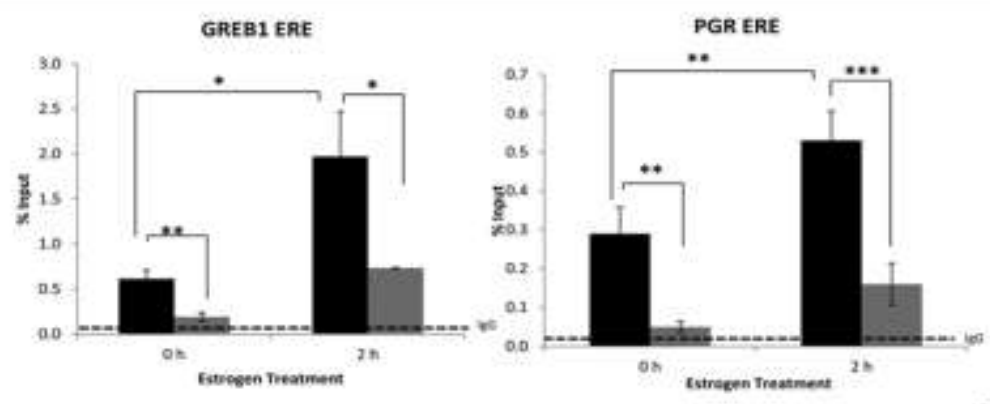

B

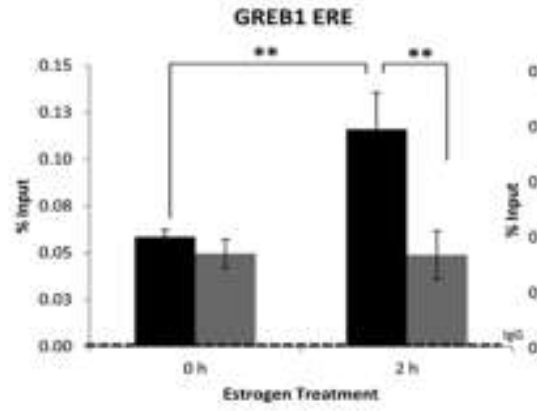

C
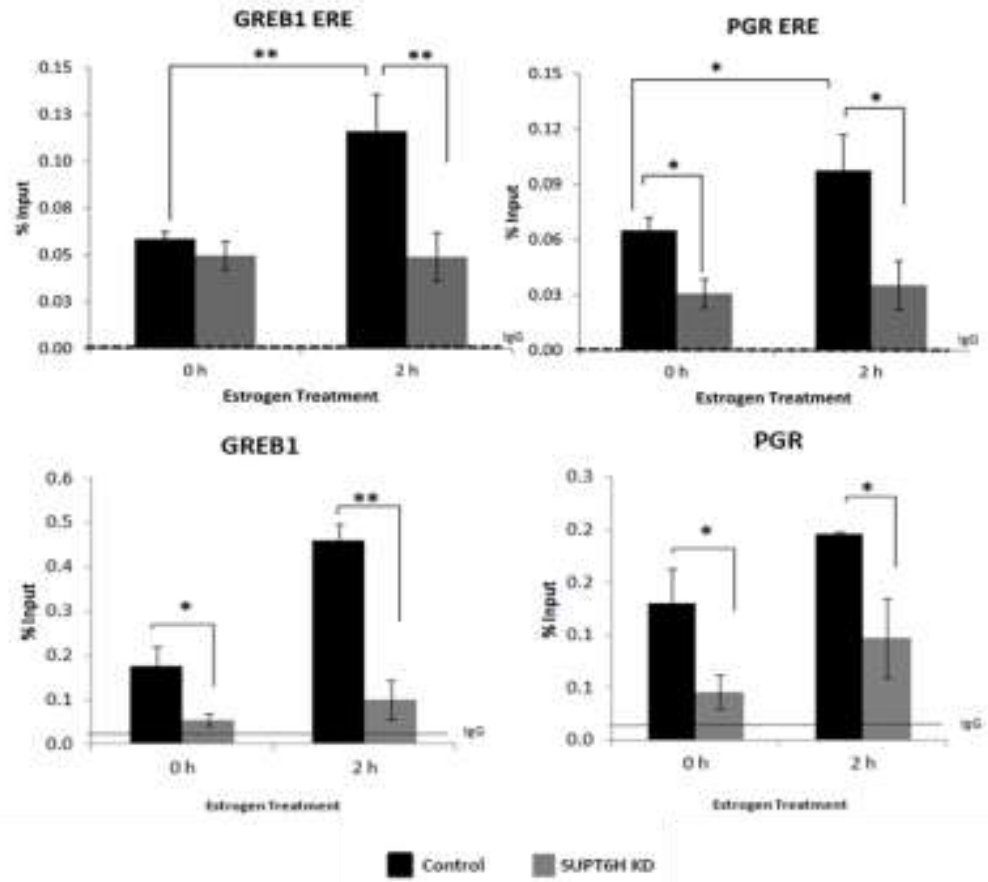

PGR

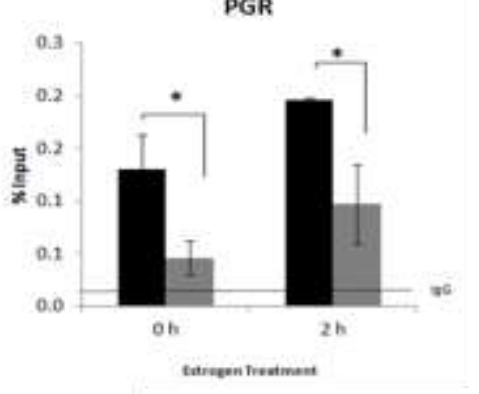

ISUPTEA ND

Fig. 9. (A and B) Decreased ERa and SUPT6H recruitment to distal EREs of GREB1 and PGR upon SUPT6H knockdown. (C) SUPT6H recruitment on the 3' ends of estrogen target genes, GREB1 and PGR. MCF7 cells were transfected and cultured as in Fig. 7A, except that cells were stimulated with $10 \mathrm{nmol} / \mathrm{L} 17 \beta$-estradiol (E2) for $2 \mathrm{~h}$. ChIP samples were normalized to input samples and expressed as "\% Input"; mean values + s.d., $n=3$. The dotted line indicates the background binding as measured by the average signal of non-specific $\lg G$ binding across all samples and sites. For statistical significance, ANOVA test was performed and indicated by * $(p \leq 0.05),{ }^{* *}(p \leq 0.01)$ and $* * *(p \leq 0.001)$.

\subsubsection{SUPT6H alters chromatin structure}

SUPT6H interacts directly with histone $\mathrm{H} 3$ and promotes chromatin assembly and/or disassembly during transcription (Bortvin and Winston, 1996). Micrococcal nuclease (MNase) digestion provides information about global chromatin openness based on the accessibility to the MNase enzyme. In order to test whether SUPT6H knockdown affects global chromatin accessibility, MNase assay was performed on chromatin 
isolated from control and SUPT6H siRNA transfected cells. The nucleosome profile indicated a more open chromatin state following SUPT6H knockdown (Fig. 10A). Quantitative analysis (Fig. 10B) showed that the percentage of mononucleosomes was significantly higher following SUPT6H knockdown (Fig. 10C) thereby indicating a more decondensed state of chromatin in the absence of SUPT6H. Thus, SUPT6H likely plays a critical role not only in chromatin disassembly during transcriptional elongation but also in reassembling nucleosomes after transcription in order to maintain an organized chromatin structure.

A

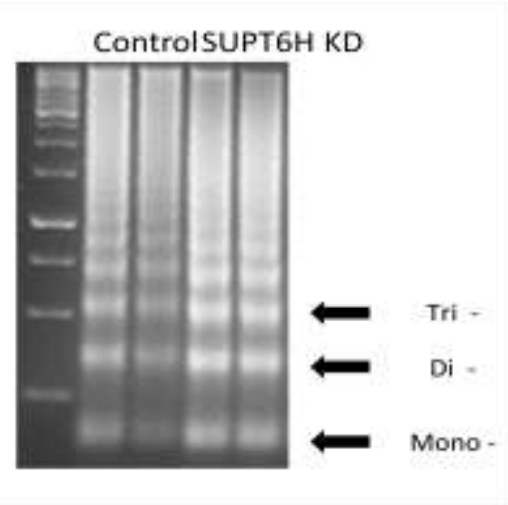

C

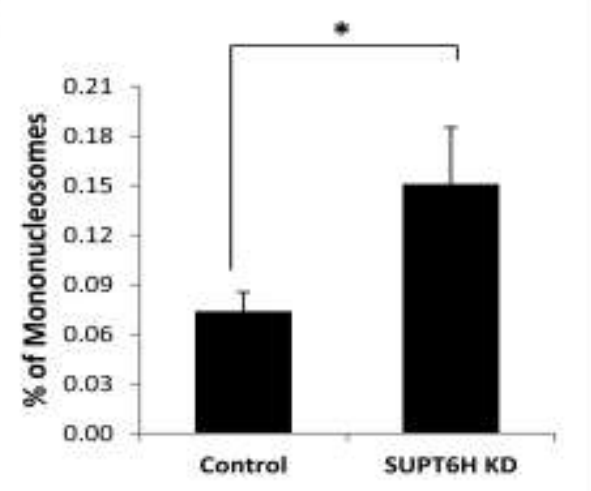

B

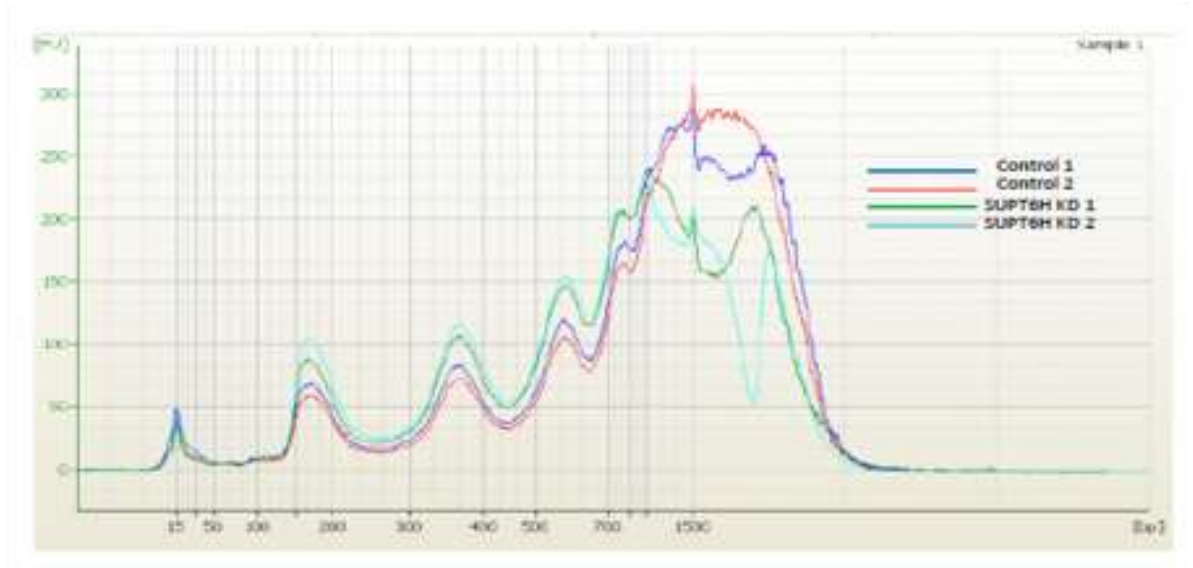

Fig. 10. Changes in chromatin structure upon SUPT6H knockdown as determined by Micrococcal Nuclease digestion. Nuclei from cells transfected with control or SUPT6H siRNAs for $48 \mathrm{~h}$ were digested with Micrococcal Nuclease for $4 \mathrm{~min}$. (A) A portion of the samples were run on agarose gel and stained with ethidium bromide. (B) MNase samples were also analyzed with a Bioanalyzer and the nucleosome profile was plotted. (C) The percentage of mononucleosomes was calculated from the nucleosome profile using the Bioanalyzer software. For statistical significance, ANOVA test was performed and indicated by * $(p \leq 0.05)$. 


\subsubsection{SUPT6H is required for maintaining H2B monoubiquitination}

During transcription elongation, P-Ser2 RNA Polymerase II plays an important role as a platform for recruiting the WAC/RNF20/RNF40 complex (Pirngruber et al. 2009; Johnsen 2012; Zhang and Yu 2011). RNF40 is an essential component of the E3 ubiquitin ligase complex responsible for $\mathrm{H} 2 \mathrm{~B}$ monoubiquitination in the transcribed region of active genes (Kim et al. 2009; Pirngruber et al. 2009). Since SUPT6H also directly interacts with P-Ser2 RNA Polymerase II during transcription elongation, we tested whether SUPT6H also influences H2Bub1. Indeed, SUPT6H knockdown led to a significant decrease in H2Bub1 levels in ERa-positive MCF7 breast cancer cells (Fig. 11A, left panel). Surprisingly, RNF40 protein levels also significantly decreased following SUPT6H knockdown. This effect does not appear to be primarily due to a decrease in RNF4O gene expression since only a modest effect of SUPT6H depletion was observed on RNF40 mRNA levels (Fig. 11A, right panel). To examine if the effect of SUPT6H depletion on H2Bub1 was ERa dependent, the ERa-negative normal mammary epithelial cell line, MCF10A was used. Similar effects on RNF40 and H2Bub1 were observed in MCF10A cells following SUPT6H knockdown (Fig. 11B). In order to determine if SUPT6H is present in complexes with ERa, RNF20 and RNF40, we performed immunoprecipitation studies in MCF7 cells using antibodies against each protein and tested for the (co-)immunoprecipitation of RNF40 and SUPT6H. Immunoprecipitation of RNF20, SUPT6H and to a lesser extent ERa resulted in a co-immunoprecipitation of RNF40 (Fig. 11C). Importantly, SUPT6H was also co-immunoprecipitated with ERa, RNF20 and to a lesser extent RNF40 suggesting that these proteins may form functional complexes in vivo. Interaction of SUPT6H with ERa is consistent with the recruitment of SUPT6H to 
distal EREs in an estrogen-dependent manner and could explain the impaired ERa recruitment to ERE of ERa target genes upon SUPT6H knockdown (Fig. 9A).

A

MCF7
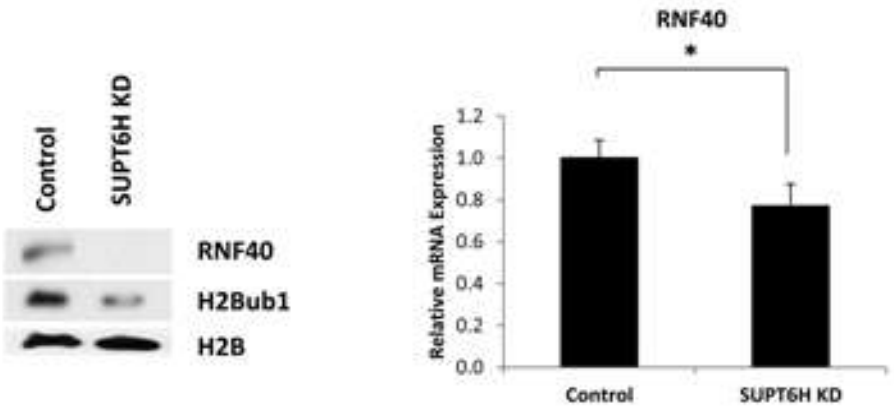

B

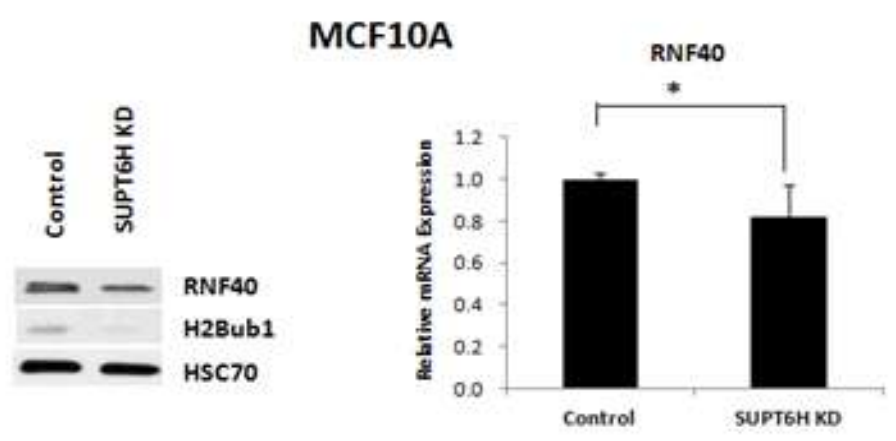

C

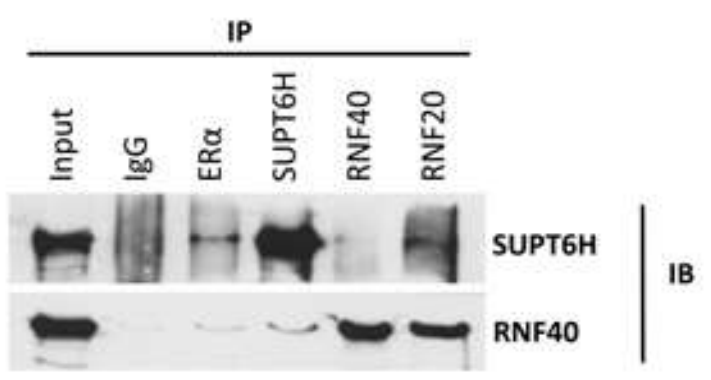

Fig. 11. SUPT6H knockdown decreases RNF40 protein levels and H2Bub1. (A) Decreased RNF40 protein and H2Bub1 levels were observed by Western blot upon SUPT6H knockdown in MCF7 cells while RNF40 mRNA levels were only modestly affected. MCF7 cells were transfected with SUPT6H siRNA for $48 \mathrm{~h}$ and then harvested for RNA and protein. (B) SUPT6H knockdown also affects RNF40 and H2Bub1 levels in ERa negative breast cancer MCF10A (mammary epithelial) cells. RNF40 mRNA levels were modestly affected with SUPT6H knockdown. MCF10A cells transfected with control or SUPT6H siRNAs were grown for $48 \mathrm{~h}$ and then analyzed by Western blot and $\mathrm{qPCR}$. Gene expression levels were normalized to $18 \mathrm{~S}$ ribosomal mRNA, graphed relative to the control sample and expressed as "Relative mRNA Expression"; mean values + s.d., $n=3$. (C) SUPT6H forms complexes with ERa, RNF20 and RNF40. Cell lysates from MCF7 cells grown in complete medium were immunoprecipitated using a non-specific IgG as a negative control or antibodies against ERa, RNF20, RNF40 and SUPT6H and were detected by Western blot using antibodies against SUPT6H and RNF40. 
To further confirm the regulation of H2Bub1 by SUPT6H, ChIP assay was performed for H2Bub1. Consistent with the decreased transcription of these genes, SUPT6H silencing in MCF7 cells decreased H2Bub1 levels in the transcribed region of the ERa target genes (CXCL12, GREB1, PGR and TFF1) (Fig. 12). These findings are also consistent with the role of $\mathrm{H} 2 \mathrm{Bub1}$ in controlling ERa activity in MCF7 cells (Prenzel et al., 2011) and support a role for SUPT6H in controlling H2Bub1 levels via interaction with the RNF20/40 complex.

\section{H2Bub1}
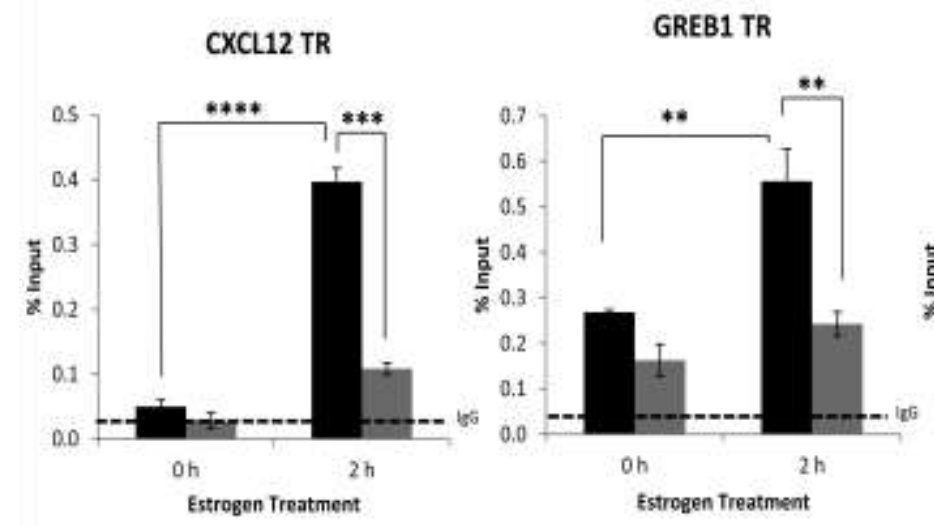

\section{GREB1 TR}

PGR TR TFF1 TR

Control
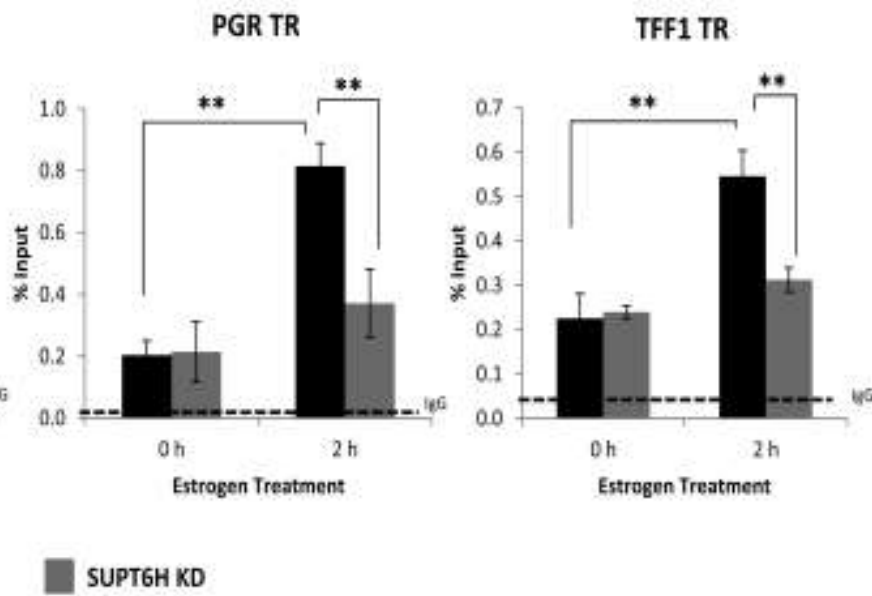

Fig. 12. Decreased H2Bub1 on transcribed region of CXCL12, GREB1, PGR and TFF1 upon SUPT6H knockdown. ChIP extracts of MCF7 cells from Fig. 9 were used. ChIP samples were normalized to input samples and expressed as "\% Input"; mean values + s.d., $n=3$. The dotted line indicates the background binding as measured by the average signal of non-specific lgG binding across all samples and sites. For statistical significance, ANOVA test was performed and indicated by ${ }^{*}(p \leq 0.05),{ }^{* *}(p \leq 0.01),{ }^{* * *}(p \leq 0.001)$ and ${ }^{* * * *}(p \leq 0.0001)$.

\subsubsection{SUPT6H expression and H2Bub1 levels are inversely correlated with breast cancer malignancy}

Previous work from our group identified significantly decreased H2Bub1 levels in malignant and metastatic breast cancers in comparison to normal mammary 
epithelium (Prenzel et al., 2011). Therefore, we performed immunohistochemical analysis of SUPT6H expression and H2Bub1 in normal human breast tissue and tumor sections as well as SUPT6H expression in the tissue microarrays analyzed in the previous study (Prenzel et al., 2011). While normal human breast epithelium exhibited detectable H2Bub1 and SUPT6H levels, ERa-positive tumors demonstrated a grade-dependent decrease in both H2Bub1 and SUPT6H (Fig. 13A). The nuclear staining intensity of SUPT6H and H2Bub1 in these and other tumor samples was quantified using digital image analysis. Box plot analysis of the intensity of both SUPT6H and H2Bub1 on the basis of various grades also indicated a grade-dependent decrease which was further confirmed with the 2D plot of the intensity in various grades (Fig. 13B and C). Moreover, both proteins inversely correlated with malignancy where normal breast samples showed significant staining of both H2Bub1 and SUPT6H while invasive ductal carcinoma samples showed significantly reduced levels (Fig. 13D). Box plot analysis further confirmed a decreased IHC-staining intensity of both SUPT6H and H2Bub1 in the nuclei of invasive ductal carcinoma in comparison to normal mammary epithelium (Fig. 13E). 
A
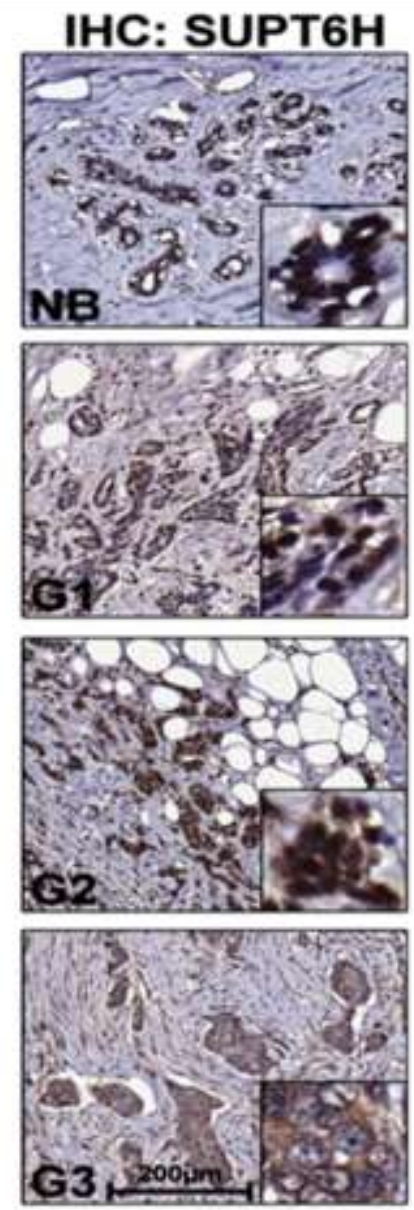

IHC: H2Bub1
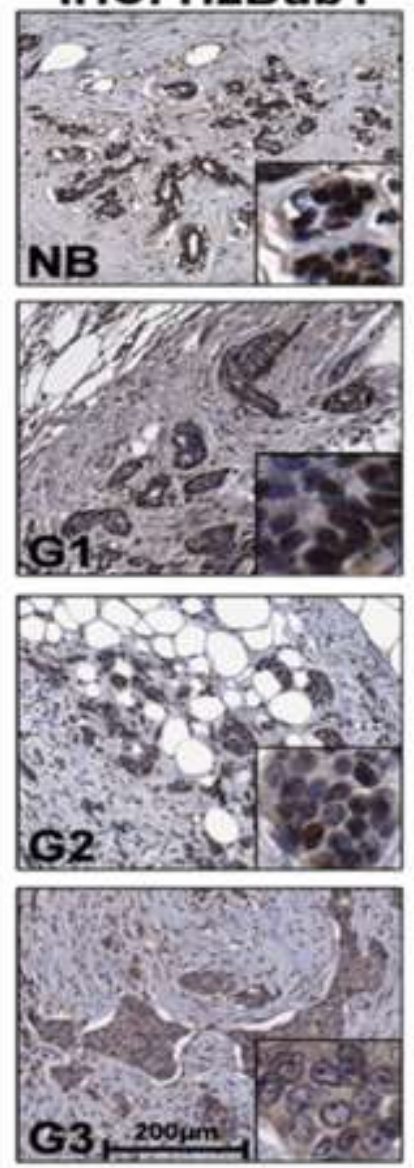

D

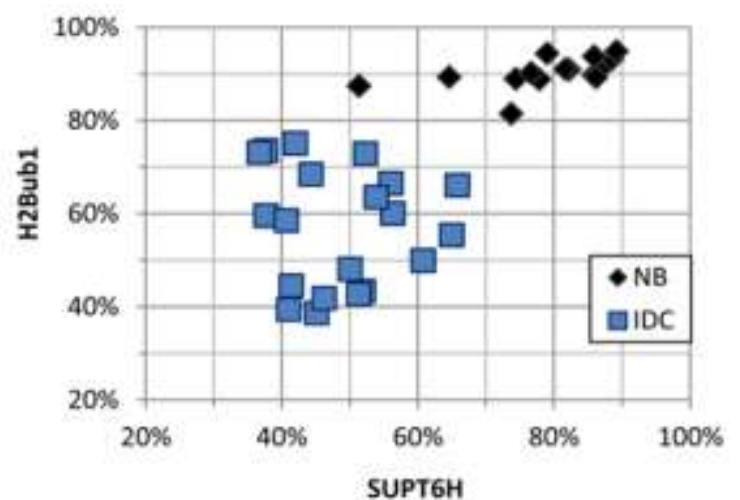

B

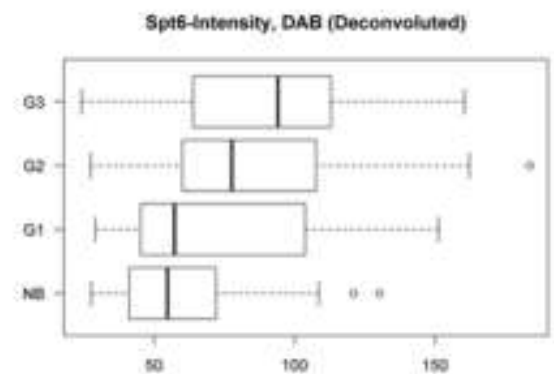

MzBub 1 - intensity, DAB (Deconvoluted)

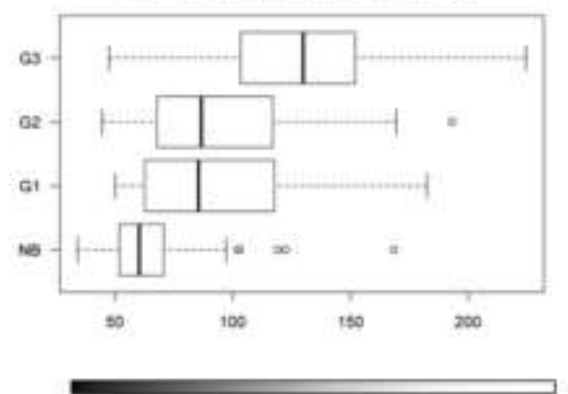

C

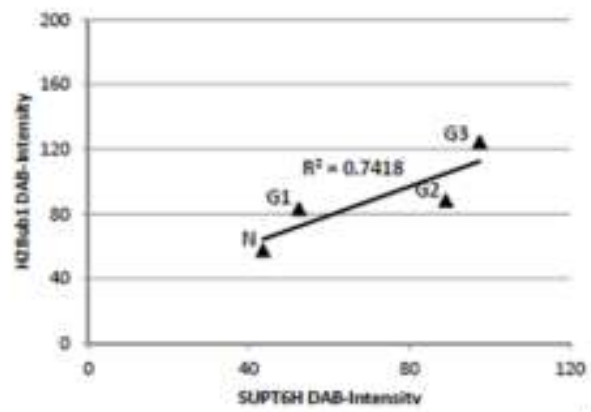

E

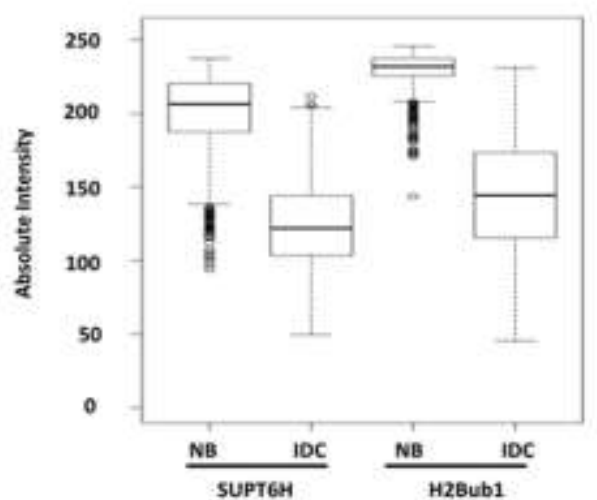

Fig. 13. Immunohistochemical analysis of human breast tissue and breast cancer sections suggests an inverse correlation between SUPT6H protein and H2Bub1 levels and malignancy.

(A) Examples of one representative normal breast specimen and ER-positive tumors of varying grades demonstrating decreasing SUPT6H and H2Bub1 protein levels with decreasing tumor differentiation status (increased tumor grade). Immunohistochemical detection of SUPT6H and H2Bub1 using DAB (brown) and Haematoxyline counterstain (blue), images 200x, magnified inserts. (NB, normal breast; G1, Grade 1; G2, Grade 2; G3, Grade 3). (B) Intensity gradient and (C) 2D plot of SUPT6H and H2Bub1 and in tissue sections divided into various grades. (D) 2D plot of SUPT6H and H2Bub1 relative staining intensity in 35 tissue samples. 15 tissue microarray cores with normal breast tissue ('NB') and 20 cores with invasive ductal carcinoma ('IDC') were quantified by digital image 
analysis (Points: Median values of 50 nuclei per core.). (E) Boxplots summarizing the intensity values of the 35 samples quantitated in (D). SUPT6H and H2Bub1 are significantly decreased in IDC compared to NB $(p<0.001)$. The analysis was done by Dr. Andreas Scheel, Pathologie Nord Hessen, Kassel.

However, this effect does not appear to be solely dependent upon ERa status since other tumor classes (eg. triple negative and Her2-positive) also showed a correlation between increased tumor grade and decreased H2Bub1 and SUPT6H. The nuclear staining intensity of SUPT6H and H2Bub1 was determined in individual tissue sections with their hormone receptor status (Fig. 14A). Box plot analysis of SUPT6H and H2Bub1 intensity in tissue sections based on their hormone receptor status showed a similar decrease in SUPT6H and H2Bub1, irrespective of ERa status (Fig. 14B). In addition, we examined whether SUPT6H mRNA levels are also decreased in breast cancer vs. normal samples. Indeed, SUPT6H mRNA levels were significantly lower in malignant breast cancer vs. normal samples in three independent studies (Finak et al., 2008); (Richardson et al., 2006)(Sørlie et al., 2001), further supporting that decreased SUPT6H is a common occurrence in breast cancer (Fig. 14C). 
A

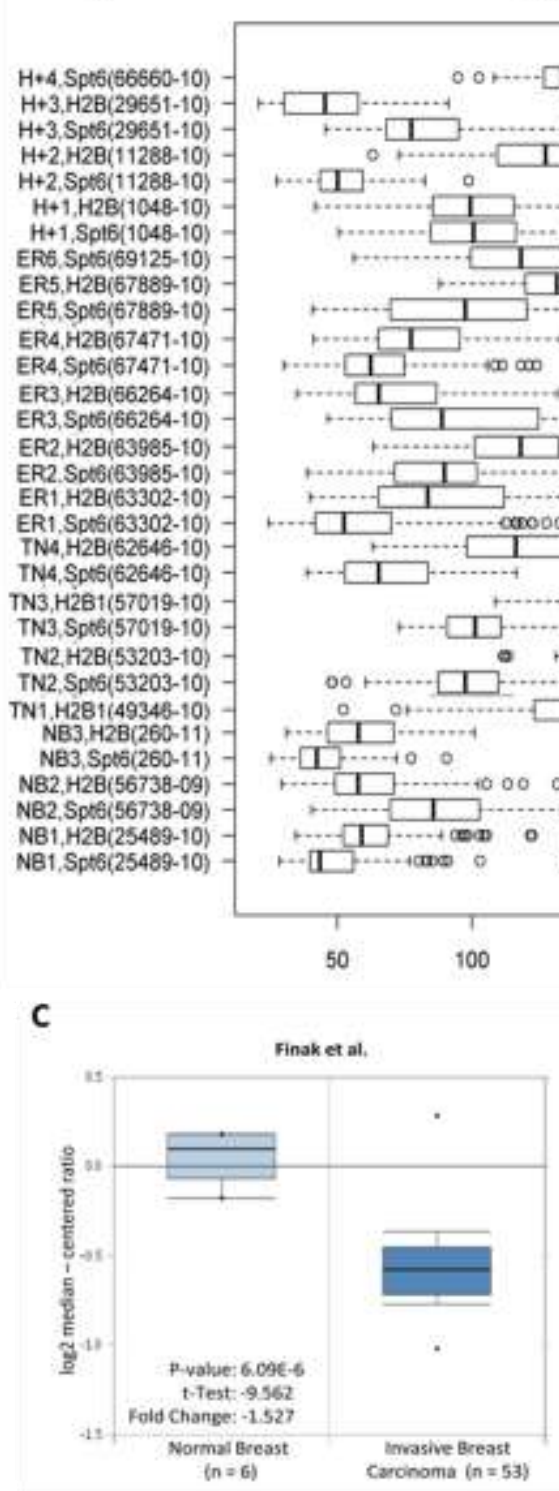

DAB-Intensity (Deconvoluted)

B
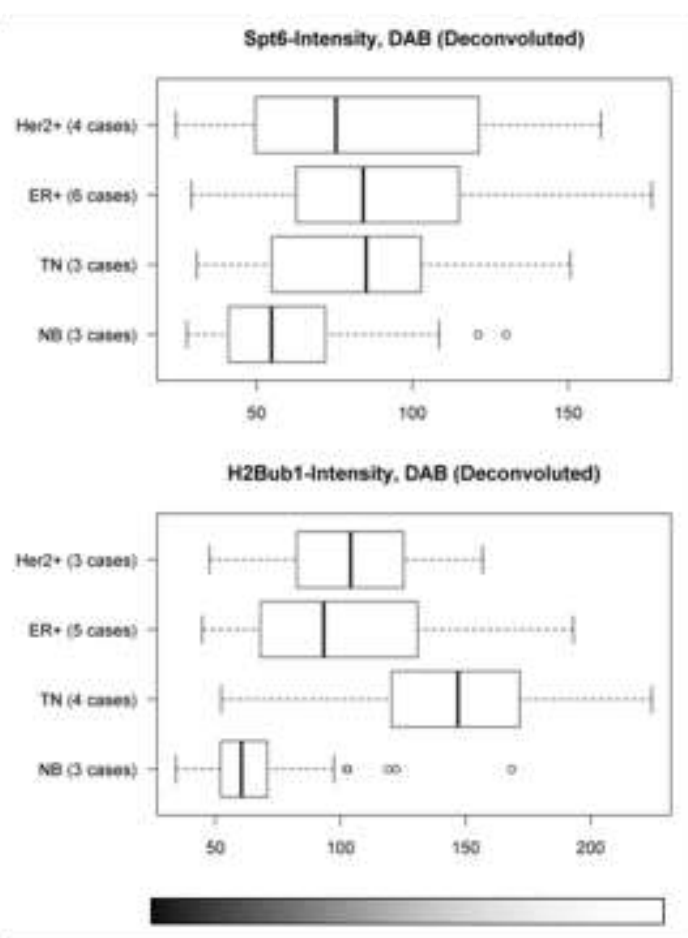

$\begin{array}{lll}1 & 1 & 250\end{array}$
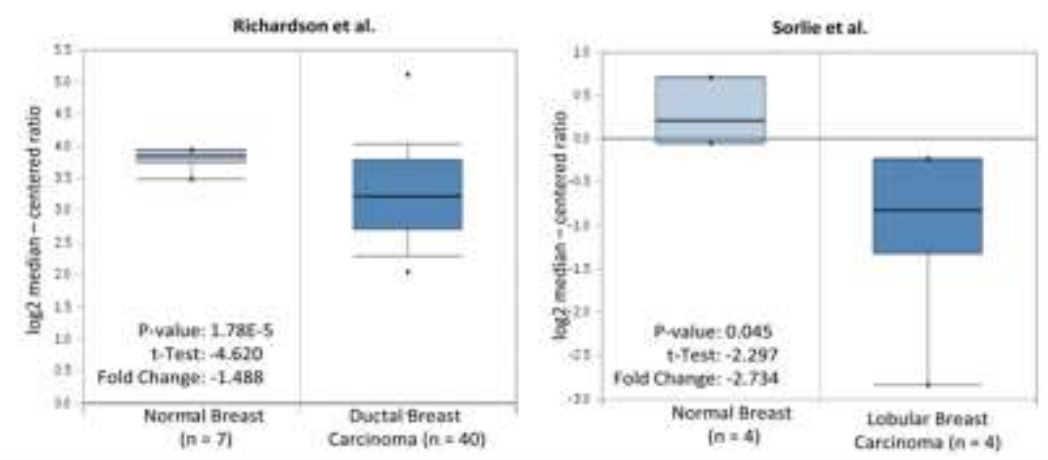

Fig. 14. Immunohistochemical analysis of human breast tissue sections based on hormone receptor status. (A) Staining intensities of SUPT6H and H2Bub1 in individual tissue sections with hormone receptor status. (B) Overall intensity gradient of SUPT6H and H2Bub1 in tissue sections classified on the basis of receptor status. (C) Gene expression analysis using the Oncomine database demonstrate decreased SUPT6H mRNA levels in three independent datasets in breast cancer vs. normal tissues.

Consistent with a central role for ERa in maintaining a luminal epithelial phenotype, treatment of T47D cells with the pure ERa antagonist, ICI 182780 resulted in upregulation of the myoepithelial marker, a smooth muscle actin (ACTA2) and downregulation of the luminal epithelial marker, cytokeratin 19 (CK19) (Fig. 15A). 
Importantly, SUPT6H depletion resulted in a similar phenotype in both MCF7 and T47D cells (Fig. 15B) suggesting that SUPT6H plays a central role in the ERadependent maintenance of a differentiated mammary luminal epithelial phenotype.

A

ACTA2

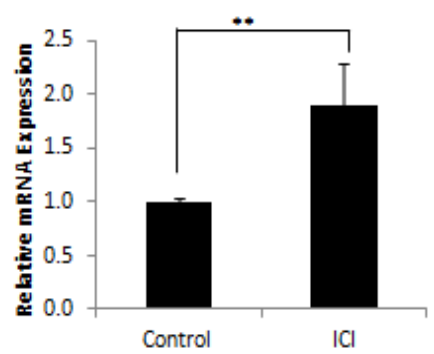

B

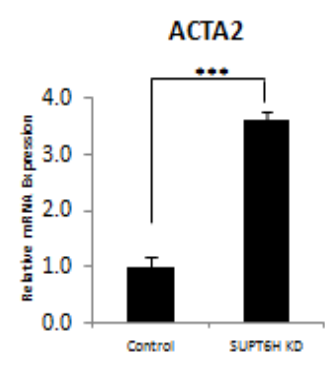

$\underline{\text { MCF7 }}$

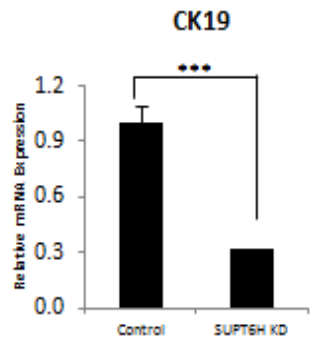

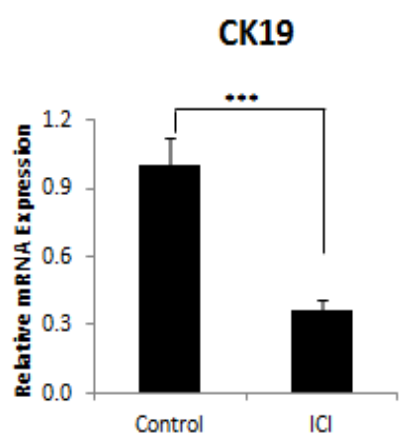

$\underline{\mathrm{T} 47 \mathrm{D}}$
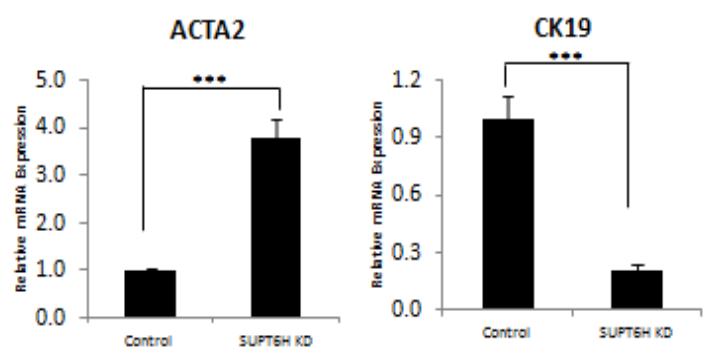

Fig. 15. SUPT6H regulates ERa-dependent luminal epithelial phenotype. (A) Expression levels of ACTA2 and CK19 in T47D cells transfected with control or treated with ICI 182780, grown for $24 \mathrm{~h}$ before switching to hormone-deprived medium and grown for another $24 \mathrm{~h}$. Cells were then stimulated with $10 \mathrm{nmol} / \mathrm{L} 17 \beta$-estradiol (E2) for $48 \mathrm{~h}$ and the expression levels of ACTA2 and CK19 were analyzed by qPCR. (B) Expression levels of ACTA2 and CK19 were analyzed in MCF7 and T47D cells transfected with control or SUPT6H siRNAs, grown for $24 \mathrm{~h}$ before switching to hormonedeprived medium and grown for another $24 \mathrm{~h}$. Cells were then stimulated with $10 \mathrm{nmol} / \mathrm{L} 17 \beta$-estradiol (E2) for $48 \mathrm{~h}$ (T47D) and 6h (MCF7) and the expression levels of ACTA2 and CK19 were analyzed by qPCR. Gene expression levels were normalized to $18 \mathrm{~S}$ ribosomal RNA, graphed relative to the control sample and expressed as "Relative mRNA Expression"; mean values + s.d., $n=3$. For statistical significance, ANOVA test was performed and indicated by ${ }^{* *}(p \leq 0.01)$ and ${ }^{* * *}(p \leq 0.001)$.

\subsubsection{SUPT6H is required for cellular differentiation}

Since the decreased levels of H2Bub1 and SUPT6H in poorly differentiated tumors compared to normal mammary epithelium and more differentiated low grade tumors did not appear to solely depend upon ERa activity, we hypothesized that SUPT6H 
may be more generally required for establishing or maintaining a differentiated phenotype. Consistent with this notion, our group previously showed that H2Bub1 increases during differentiation in various systems and that perturbation of the H2Bub1 regulatory pathway significantly impairs differentiation (Karpiuk et al. 2012; (Fuchs et al., 2012) Chen et al. 2012). A survey of gene expression correlations in the GeneSapiens database (Kilpinen et al., 2008) identified a significant correlation between SUPT6H and RNF4O gene expression in adipose tissue and human mesenchymal stem cells (hMSCs) suggesting that SUPT6H may be intimately associated with the H2Bub1 regulatory pathway in normal differentiating cells of the mesenchymal lineage (Fig. 16A). Given the established role of H2Bub1 in controlling multipotent differentiation of hMSCs, we performed siRNA-mediated knockdown of SUPT6H in the immortalized hMSC-Tert20 cell line (Simonsen et al., 2002) and examined its effects on cellular differentiation. Consistent with a role for SUPT6H in controlling H2B monoubiquitination, H2Bub1 levels were decreased in differentiating hMSCs following SUPT6H knockdown (Fig. 16B). We further examined the effects of SUPT6H depletion on differentiation into the adipocyte and osteoblast lineages. Similar to the effects observed following knockdown of components of the H2Bub1 regulatory pathway (Karpiuk et al., 2012b), SUPT6H knockdown prevented both adipocyte and osteoblast differentiation as displayed by a near complete loss of Oil Red $\mathrm{O}$ and alkaline phosphatase staining, respectively, following the induction of differentiation (Fig. 16C and D). Importantly, SUPT6H knockdown also resulted in decreased expression of adipocyte- (PDK4, PPARG, RASD1; Fig. 16E) and osteoblast-specific (RUNX2; Fig. 16F) marker genes. Thus, SUPT6H is essential for hMSC differentiation, probably in part via the H2Bub1 regulatory pathway. 


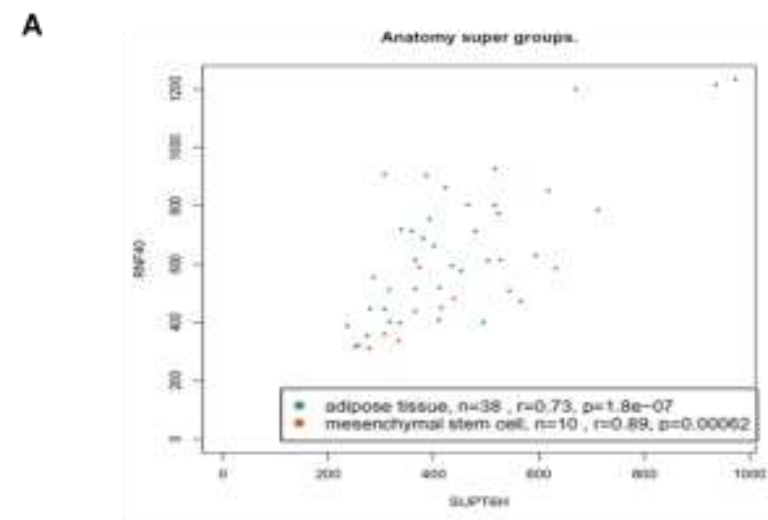

B

C
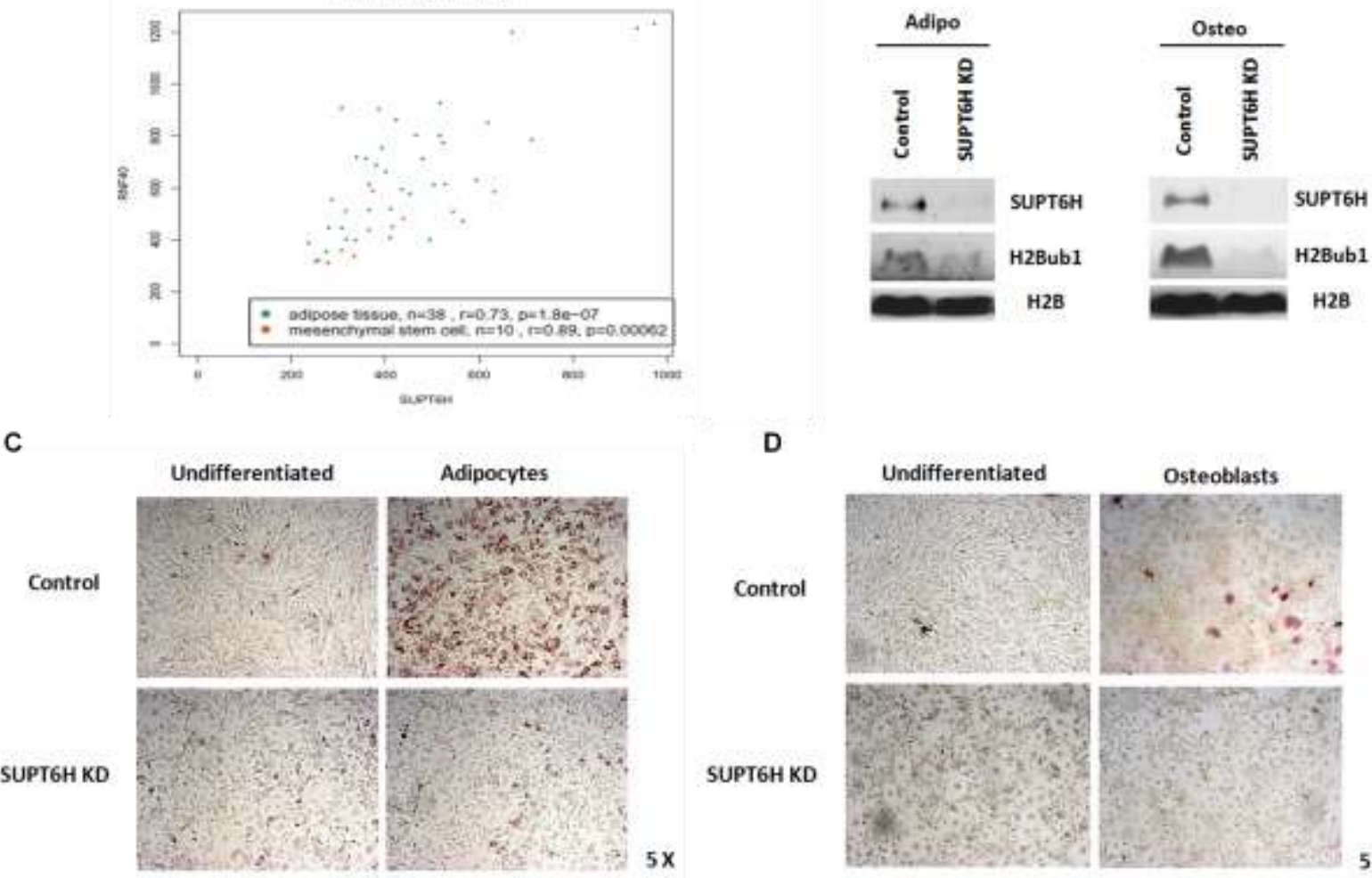

D
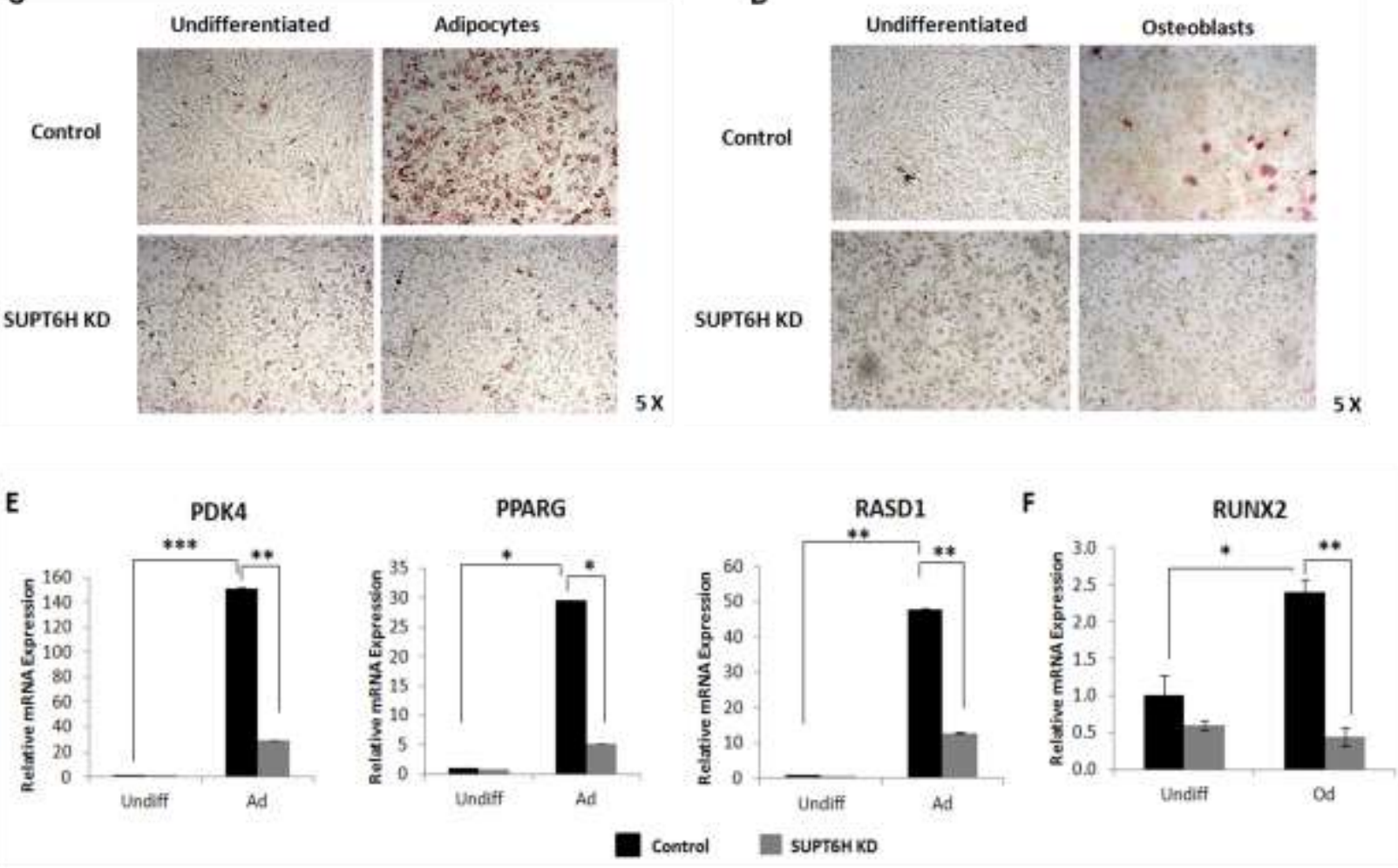

Fig. 16. SUPT6H knockdown impairs hMSC differentiation to the adipocyte and osteoblast lineages. (A) Correlation between SUPT6H and RNF40 mRNA levels in mesenchymal stem cells and adipose tissue as analyzed using the GeneSapiens database. (B-F) hMSCs transfected with control or SUPT6H siRNAs were induced to differentiate into adipocytes and osteoblasts for 5 days. (B) Protein extracts were analyzed by Western blot using antibodies against SUPT6H, H2Bub1 and H2B as a loading control. (C) Differentiation was examined using Oil Red $O$ staining for adipocytes and (D) alkaline phosphatase staining for osteoblast following SUPT6H knockdown. (E and F) Decreased expression of adipocyte differentiation genes, PDK4, PPARG and RASD1, and RUNX2 for osteoblast differentiation was found upon SUPT6H knockdown. The expression levels were normalized to HNRNPK, graphed relative to the control sample and expressed as "Relative mRNA Expression"; mean values + s.d., $n=2$. For statistical significance, ANOVA test was performed and indicated by * $(p \leq 0.05),{ }^{* *}(p \leq 0.01)$ and ${ }^{* * *}(p \leq 0.001)$. 


\subsubsection{SUPT6H suppresses H3K27me3 on ERa- and differentiation-regulated genes}

Gene repression via trimethylation of lysine 27 of histone $\mathrm{H} 3(\mathrm{H} 3 \mathrm{~K} 27 \mathrm{me})$ plays an important role both in tumorigenesis as well as in the suppression of lineage-specific gene expression in stem cells. For example, the expression of the H3K27 methyltransferase EZH2 is associated with a more aggressive and less differentiated phenotype in breast cancer (Collett et al., 2006); (Raaphorst et al., 2003) and with tamoxifen resistance in advanced ERa-positive breast cancer (Reijm et al., 2011) implicating aberrant H3K27me3 in breast cancer progression. Recent studies have uncovered interactions between SUPT6H and both known enzymes which remove methylation from H3K27, JMJD3 and UTX (Chen et al., 2012b); Wang et al. 2013). Furthermore, SUPT6H and H2Bub1 were shown to control H3K27 demethylation during cell differentiation (Wang et al. 2013; Karpiuk et al. 2012). Thus, we hypothesized that SUPT6H may play a similar role in suppressing H3K27me3 on ERa target genes. Therefore, we performed ChIP analysis for H3K27me3 on various direct estrogen-responsive ERa target genes (CXCL12, GREB1, PGR, TFF1) following SUPT6H knockdown. In each case, H3K27me3 levels were significantly increased following SUPT6H knockdown (Fig. 17A). Since SUPT6H is a histone chaperone and interacts with histone $\mathrm{H} 3$, it was speculated that the increase in H3K27me3 could be due to increased H3 levels. Hence, ChIP analysis for total H3 was performed on estrogen-target genes (CXCL12, GREB1, PGR and TFF1) following SUPT6H knockdown and $\mathrm{H} 3$ levels were found to be unregulated (Fig. 17B). To further validate the increase in H3K27me3 levels on these genes, H3K27me3 levels were normalized with H3 levels on respective genes and were 
found to be significantly increased both in the absence and presence of estrogen

(Fig. 17C).

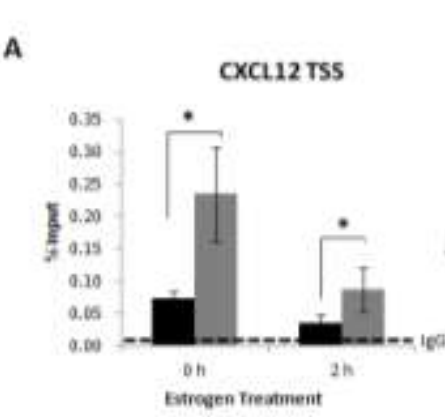

B

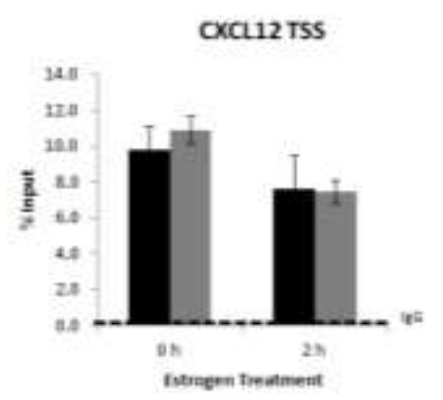

c

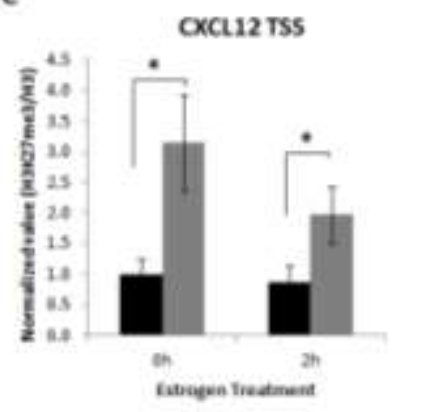

H3K27me3
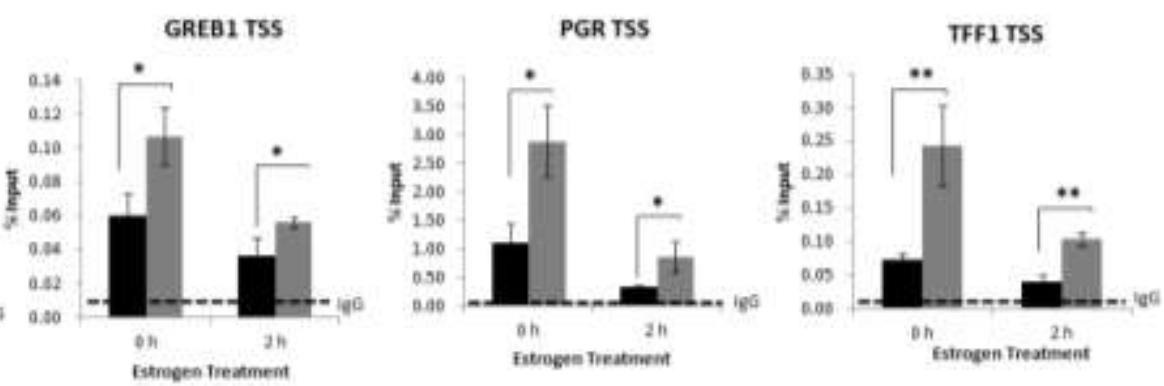

$\underline{\mathrm{H} 3}$
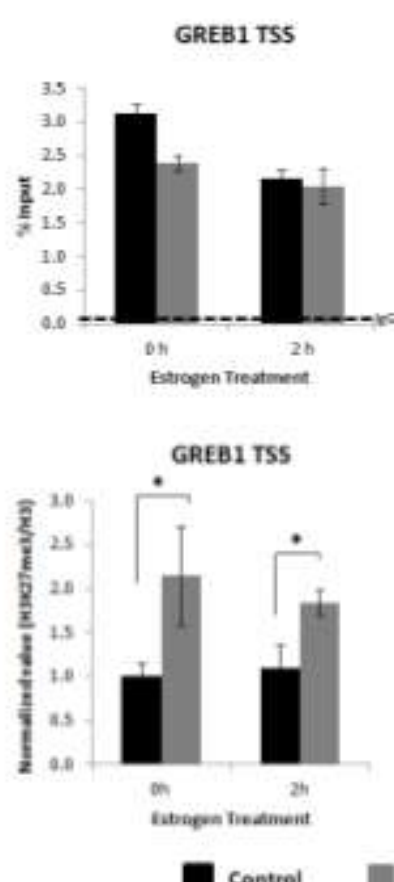
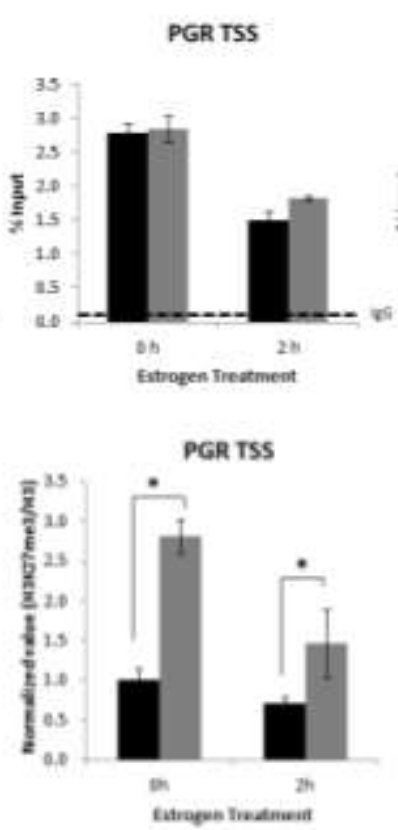

SUPT6A Ko

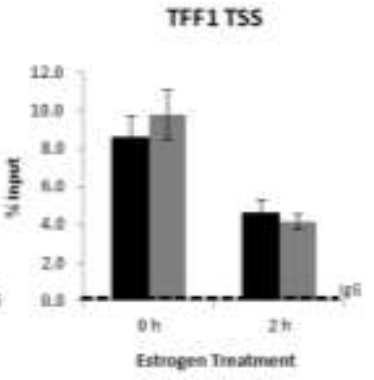

TFF1 TSS

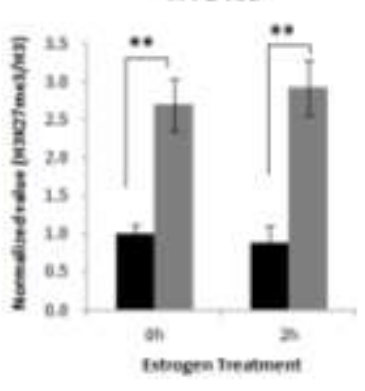

Fig. 17. SUPT6H regulates $\mathrm{H} 3 \mathrm{~K} 27 \mathrm{me} 3$ on genes during transcription. (A) Samples from MCF7 cells shown in Fig. 9 were examined for H3K27me3 occupancy near the TSS of the estrogenregulated genes CXCL12, GREB1, PGR and TFF1. (B) H3 ChIP was performed on the same MCF7 cell extracts and $\mathrm{H} 3$ levels on TSS of various estrogen target genes, CXCL12, GREB1, PGR and TFF1 were found to be unregulated. (C) H3K27me3 levels were normalized to total H3 levels. ChIP samples were normalized to input samples and expressed as "\% Input"; mean values + s.d., $n=3$. The dotted line indicates the background binding as measured by the average signal of non-specific IgG binding across all samples and sites. For statistical significance, ANOVA test was performed and indicated by ${ }^{*}(p \leq 0.05)$ and ${ }^{* *}(p \leq 0.01)$. 
Furthermore, similar to our previous results following RNF40 knockdown, SUPT6H depletion also resulted in increased H3K27me3 levels on three different H2Bub1dependent adipocyte-specific genes (PDK4, PPARG and RASD1) in both undifferentiated hMSCs and following adipocyte differentiation (Fig. 18).

\section{H3K27me3}
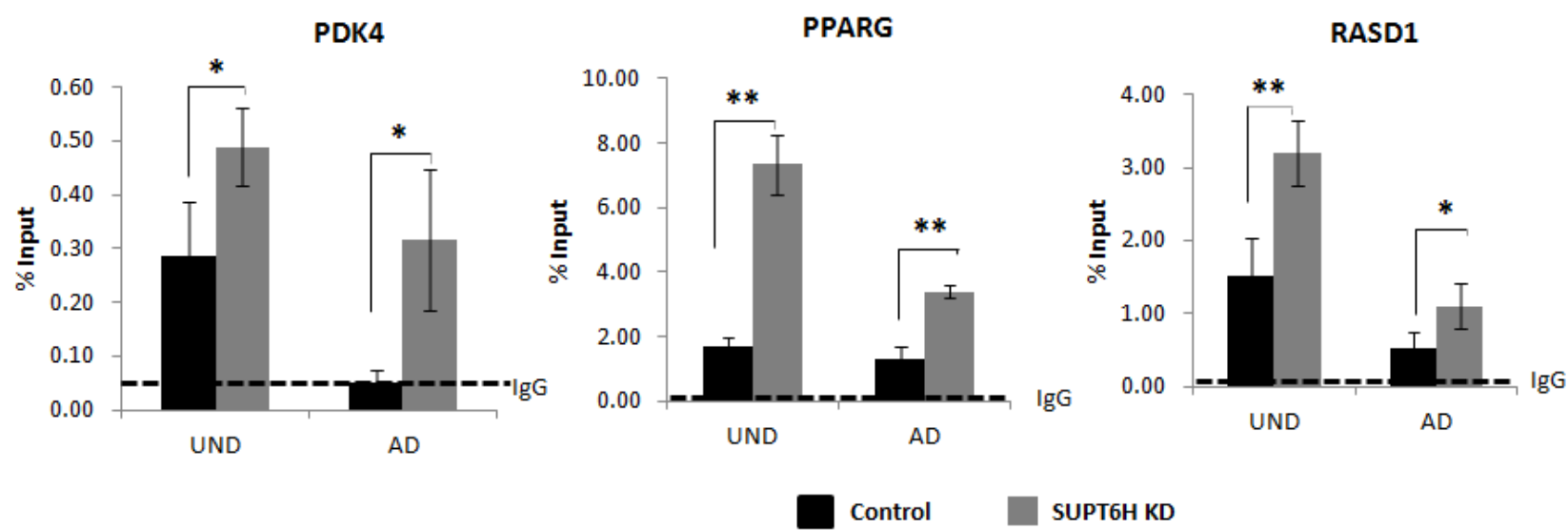

Fig. 18. SUPT6H regulates $\mathrm{H} 3 \mathrm{~K} 27 \mathrm{me} 3$ on adipocyte-specific genes during transcription. $\mathrm{hMSCs}$ were transfected with control or SUPT6H siRNA and induced to differentiate to the adipocyte lineage for 5 days as in Fig. 10. H3K27me3 occupancy was then examined on adipocyte-specific genes using chromatin immunoprecipitation. H3K27me3 levels increase on PDK4, PPARG and RASD1 genes upon SUPT6H knockdown which remain high even upon differentiation. ChIP samples were normalized to input samples and expressed as "\% Input"; mean values + s.d., $n=3$. The dotted line indicates the background binding as measured by the average signal of non-specific IgG binding across all samples and sites. For statistical significance, ANOVA test was performed and indicated by ${ }^{*}(p \leq 0.05)$ and ${ }^{* *}(p \leq 0.01)$.

The results for the above mentioned data entitled "SUPT6H Controls Estrogen Receptor Activity and Cellular Differentiation by Multiple Epigenomic Mechanisms" are in press at Oncogene.

\subsection{H2Bub1 and Mammary Stem Cell Phenotype}

The link between P-Ser2 RNA Polymerase II and H2Bub1 mediated via CDK9-WAC-

RNF20/40 was well established (Pirngruber et al. 2009; (Pirngruber et al.) Johnsen 
2012b; (Johnsen, 2012c) Karpiuk et al. 2012). BRD4 recognizes the acetylated chromatin and recruits P-TEFb to promote CTD phosphorylation of RNA Polymerase II and transcriptional elongation (Yang et al., 2005); Jang et al. 2005). During tumor progression, the differentiated cells undergo dedifferentiation and acquire stem cell characteristics. Therefore, we further examined the function of H2Bub1 and its upstream regulator, BRD4 in controlling epithelial to mesenchymal transition and the acquisition of a stem cell phenotype in breast cancer. To address these aspects, we investigated the role of $\mathrm{H} 2 \mathrm{Bub} 1$ and BRD4 in the regulation of mammary stem cell phenotype.

\subsubsection{RNA-sequencing identifies similarities in gene expression profiles following perturbation of BRD4, RNF20 or RNF40}

The effect of H2Bub1 deficiency on cells is primarily investigated by knocking down the E3 ligases, RNF20 or RNF40 (heterodimeric complex) which are responsible for H2Bub1. Likewise, the effect of BRD4 suppression could be examined using BRD4 siRNA or BRD4 inhibition by JQ1 treatment. Thus, using siRNA-mediated knockdown of RNF20, RNF40 and BRD4 for 3 days in MCF10A (normal mammary epithelial) cells, RNA and protein were isolated. The knockdown efficiency of all siRNAs was confirmed by both Western blot and GPCR before performing highthroughput sequencing of RNA samples (Fig. 19A and B). As expected, H2Bub1 protein levels decreased upon RNF20 and RNF40 knockdown (Fig. 19A, left panel). The effects of BRD4 depletion on H2Bub1 has never been investigated and for the first time, we demonstrate that H2Bub1 levels are decreased upon BRD4 knockdown and JQ1 treatment (Fig. 19A, right panel), thereby providing the basis to investigate the correlation further. 
A
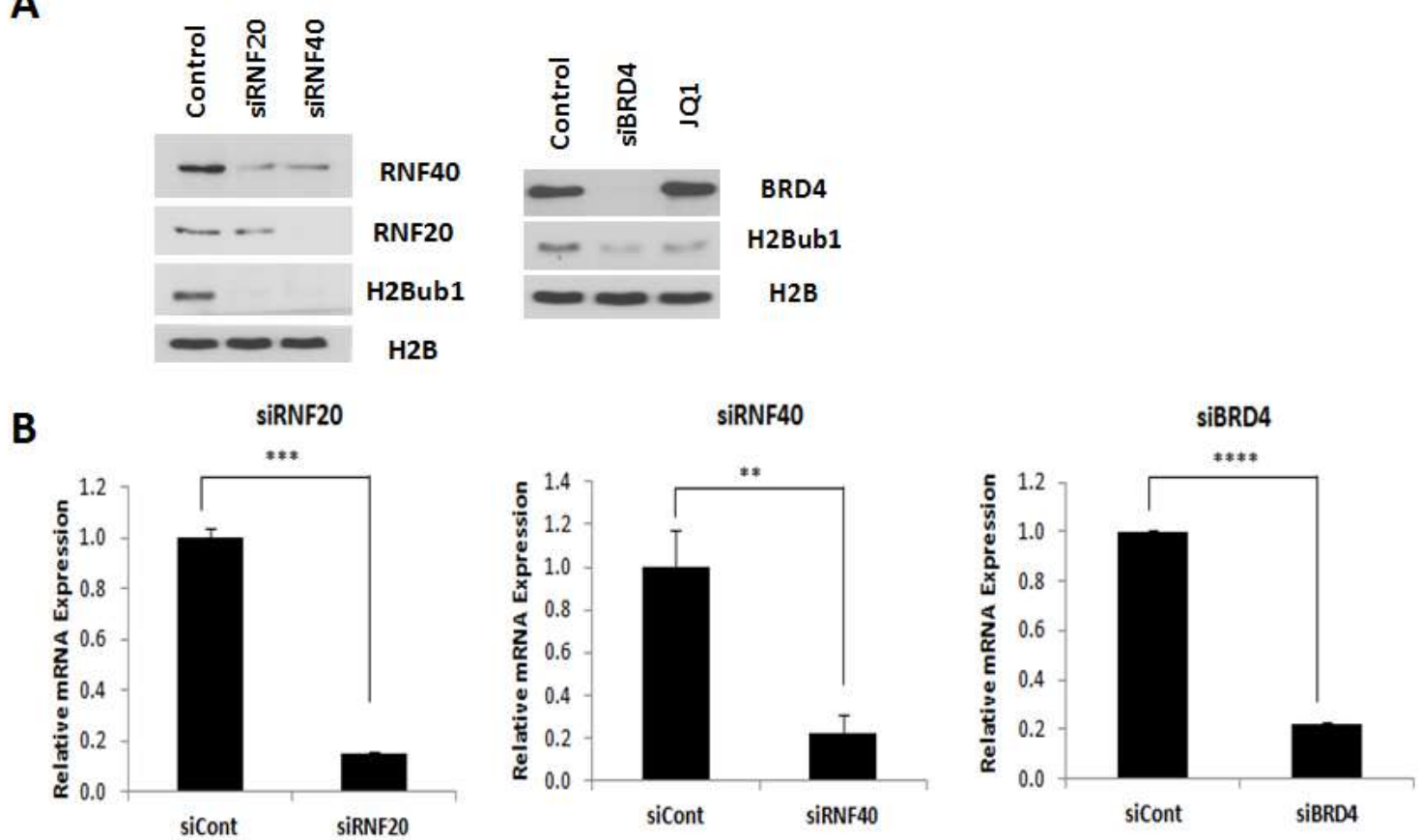

Fig. 19. BRD4 knockdown decreases H2Bub1 levels in MCF10A cells. MCF10A cells were transfected with siRNAs for Control, RNF20, RNF40 and BRD4 or treated with $250 \mathrm{nM}$ JQ1 for 3 days. (A) BRD4 suppression by either BRD4 siRNA or JQ1 inhibition decreases H2Bub1 protein levels. H2B was used as a loading control. (B) Knockdown efficiency of siRNAs was confirmed by qPCR. Gene expression levels were normalized to HNRNPK, graphed relative to the control sample and expressed as "Relative mRNA Expression"; mean values + s.d., $n=3$. For statistical significance, ANOVA test was performed and indicated by ${ }^{* *}(p \leq 0.01),{ }^{* *}(p \leq 0.001)$ and ${ }^{* * *}(p \leq 0.0001)$.

Thus, the potential link between BRD4 and H2Bub1 was further examined by sequencing RNA from these cells and analyzing the genes affected. The genes were selected based on fold regulation of greater than or equal to 2 for upregulated genes and less than or equal to 0.5 for downregulated genes with $p$-values of less than or equal to 0.05 for statistical significance. To determine significant overlap between knockdowns, genes from these subsets were further selected to have regulation of 1.2 or 0.86 fold in any knockdown. These differentially expressed genes in the knockdowns of RNF20, RNF40 and BRD4 are depicted in the heatmap (Fig. 20A) and indicate a common trend of gene regulation among all conditions. Moreover, Venn diagram demonstrated a large overlap of 374 genes regulated by RNF20, 
RNF40 and BRD4 (Fig. 20B). Consistent with the function of RNF20 and RNF40 proteins, there was a huge overlap of 446 genes in their regulated gene sets as depicted in the heatmap and Venn diagram (Fig. 20C and D). Thus, the RNA-seq analysis substantiated the hypothesis that there is a common trend of gene regulation between BRD4 and H2Bub1 pathway.

A

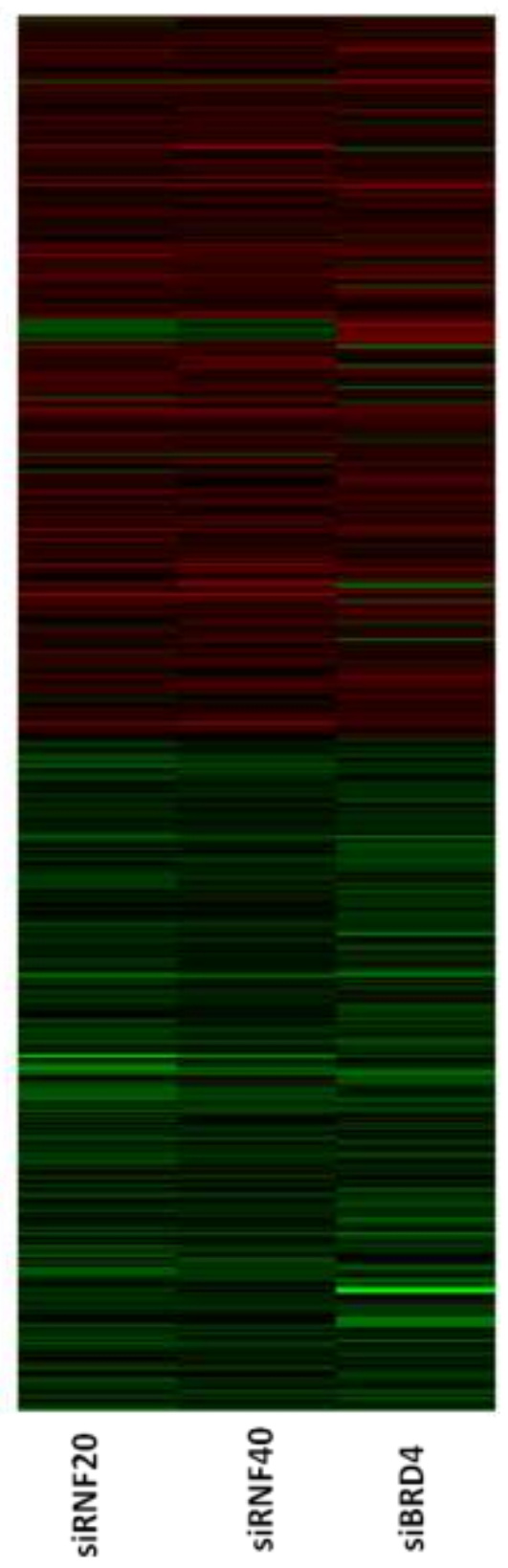

C

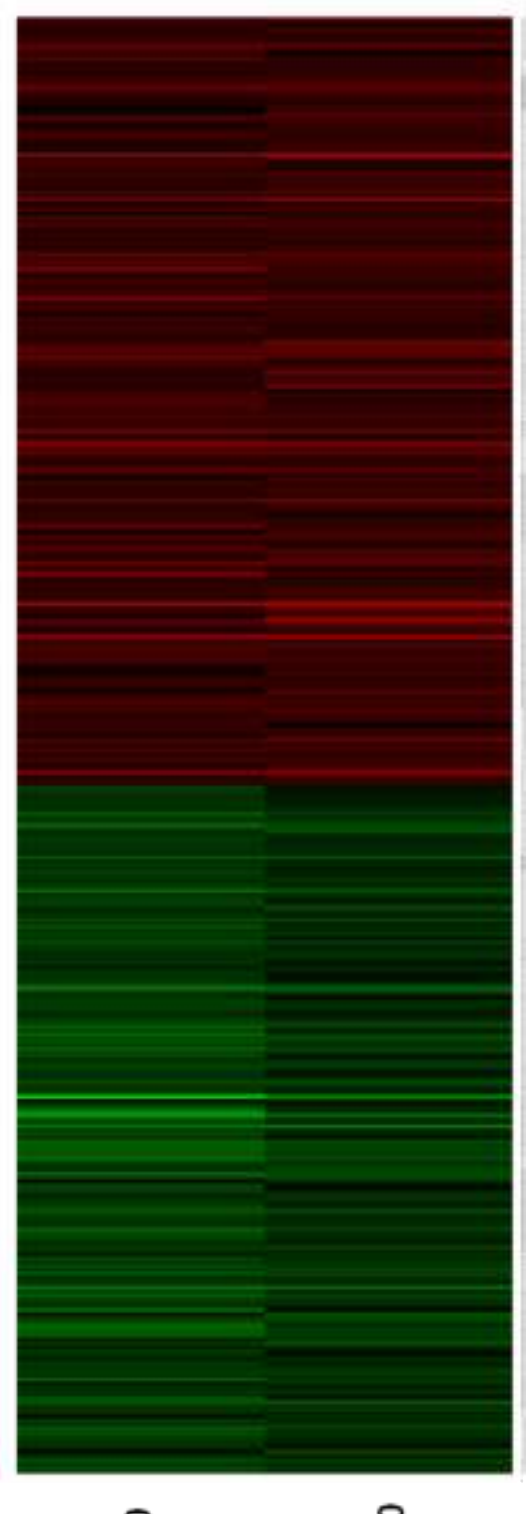

忿 


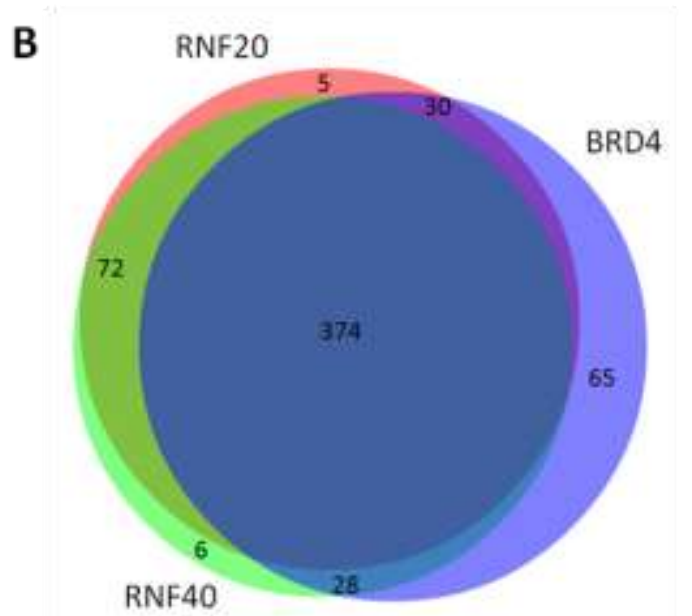

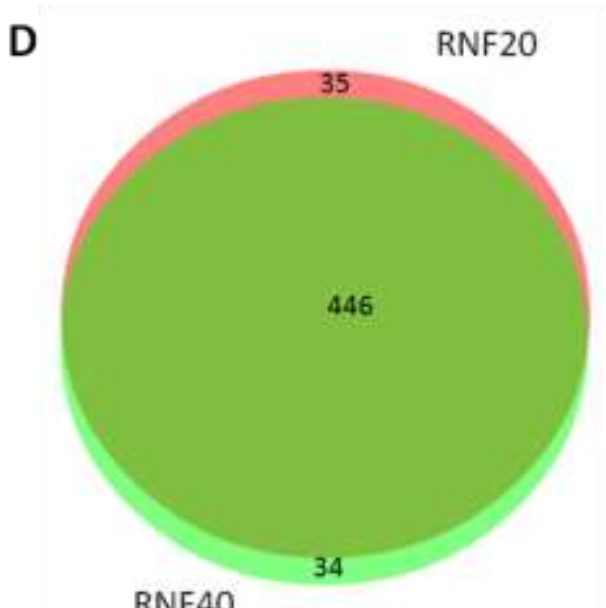

RNF40

Fig. 20. BRD4 and H2Bub1 regulate gene expression in a similar fashion. MCF10A cells were transfected with siRNAs for Control, RNF20, RNF40 and BRD4 for 3 days, RNA was harvested and used for high-throughput sequencing. Expression values were normalized to the corresponding gene in cells transfected with control siRNA. Heat maps were generated from statistically significant (padjvalues $\leq 0.05$ ) up- (red) or down- (green) regulated genes with the cut-off of -1.0 and +1.0 log2fold change in all the conditions; mean values, $n=2$. (A) Heatmap and (B) Venn diagram for RNF20, RNF40 and BRD4 regulated genes. (C) Heatmap and (D) Venn diagram for RNF20 and RNF40 regulated genes. For Venn diagrams, genes from the first cut-off subsets were further selected with cut-off of -0.26 and +0.26 logefold change in any condition.

\subsubsection{Overlap between BRD4 and H2Bub1 recruitment sites}

Since BRD4 and H2Bub1 showed similar pattern of gene regulation, we next used ChIP-seq to determine whether BRD4 and H2Bub1 play direct roles in regulating transcription of target genes. No studies have analyzed the correlation between genome-wide recruitment of BRD4 and H2Bub1. Thus, to understand the mechanistic link between BRD4 and H2Bub1 in gene regulation, ChIP-sequencing of BRD4 and H2Bub1 was performed in MCF10A cells. Using Cis-regulatory Element Annotation System (CEAS), part of cistrome package (Liu et al., 2011a), the enrichment for BRD4 on specific genomic features (eg. Promoter regions, introns etc.) could be determined. It is estimated as the relative enrichment of ChIP regions in particular genomic feature with respect to the whole genome. As shown in Fig. 21, the pie chart depicts the distribution of BRD4 recruitment on various genomic 
locations. It was observed that BRD4 is recruited to gene promoters and coding exons.

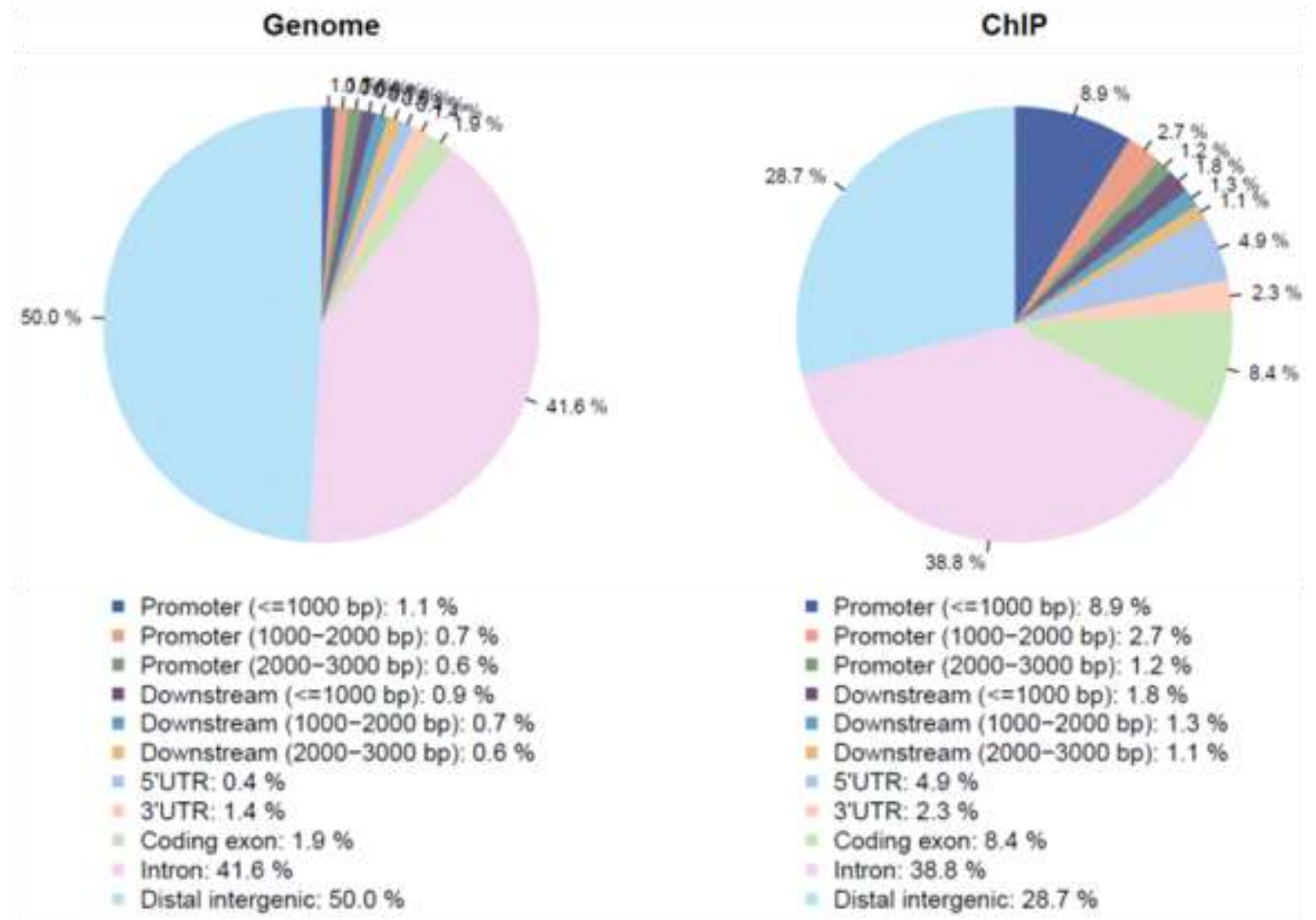

Fig. 21. BRD4 occupancy in the genome. Using CEAS, the enrichment of BRD4 at various genomic locations in Human hg19 was determined.

In order to determine the correlation between BRD4 recruitment and overall gene expression, genes were categorized based on their absolute expression levels seen in RNA-seq in control cells. Gene expression levels were based on RPKM (reads per kilobase of DNA per million reads) values where higher RPKM indicates higher expression (Mortazavi et al., 2008). The genes were then separated into categories : under 500 RPKM, 500-1500 RPKM, 1500-3000 RPKM, 3000-5000 RPKM and 500010,000 RPKM where under 500 indicates the lowest expressed genes and 500010,000 indicates the highest expressed genes. Based on this classification, average BRD4 recruitment was determined via aggregate plot analysis (using CEAS) (Liu et 
al., 2011a) to deduce the average signal profiles around transcriptional start sites of genes in the various gene expression-dependent groups. In this case, BRD4 recruitment near the transcription start site (TSS) $( \pm 3 \mathrm{~kb}$ relative to TSS) varied according to the expression levels of the genes (Fig. 22A). The highly expressed genes had more BRD4 recruitment whereas the lowly expressed genes had the least BRD4 recruitment. A similar gene expression-dependent pattern was observed for H2Bub1, further strengthening the correlation between the two (Fig. 22B). Since H2Bub1 is known to be an important histone modification coupled with transcription elongation and often associated with the transcribed region (Minsky et al., 2008b), H2Bub1 recruitment was also observed across the gene bodies (Fig. 22C). Similarly, the highly expressed genes possessed higher H2Bub1 while the lowly expressed genes had lower H2Bub1 levels in the transcribed regions. These results are consistent with the previously published data for the presence of H2Bub1 in the transcribed region (Minsky et al., 2008b). Based on the published ChIP-seq data for RNAPII (SRR488765) (Baillat et al., 2012), H3K9/14ac (SRR398030) and H3K4me3 (SRR398029) (Choe et al., 2012), aggregate plot analyses were performed on the genes classified as in Fig. 22A. Consistent with BRD4 and H2Bub1 recruitment, there was similar pattern for RNAPII, H3K9/14ac and H3K4me3 on the TSS of these genes correlating well with the expression of these genes (Fig. 22D).

A

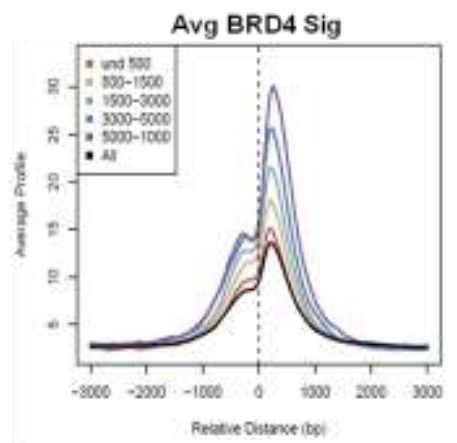

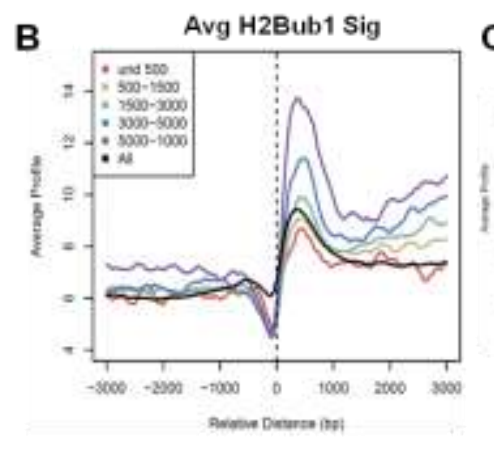

c

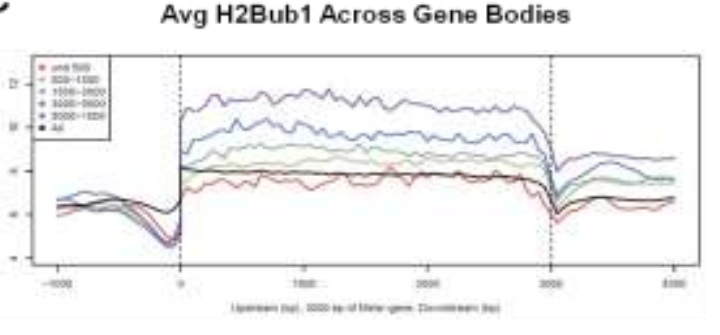


D
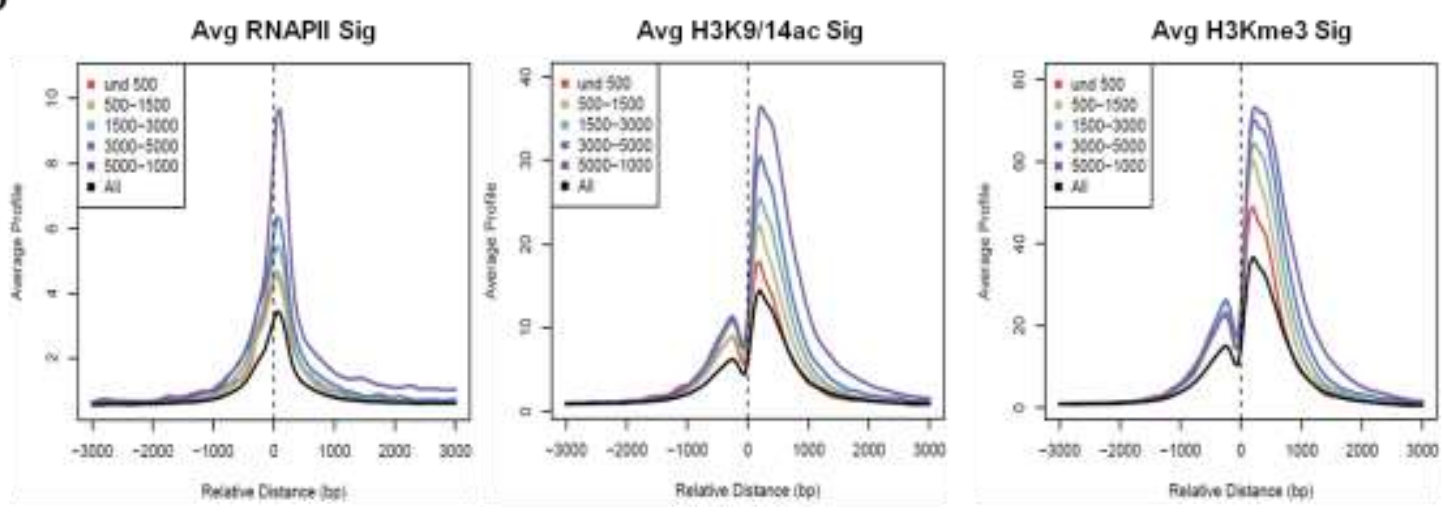

Fig. 22. BRD4 and H2Bub1 occupancy correlate with the expression of the gene. Genes were sorted into various categories based on their RPKM values from RNA seq in siCont sample. Using CEAS, the average signal intensity at TSS (3kb upstream and downstream) for the proteins or histone modifications correlating with the expression levels was evaluated. (A-B) Average BRD4 and H2Bub1 recruitment around the TSS of the genes classified on the basis of expression. (C) H2Bub1 signal across the gene bodies correlating with the gene expression. (D) Using published data for RNAPII, $\mathrm{H} 3 \mathrm{~K} 9 / 14 \mathrm{ac}$ and $\mathrm{H} 3 \mathrm{~K} 4 \mathrm{me} 3$, their occupancy correlated with the determined gene expression list. The analysis was performed by Prof. Steven A. Johnsen, UKE, Hamburg.

Heatmap analysis diagrammatically depicts the recruitment signals of ChIP-seq at various genomic locations. The genomic locations are clustered together based on kmeans clustering into clusters having similar patterns. The heatmaps illustrating the recruitment of BRD4, RNAPII, H3K4me3, H3K9/14ac and H2Bub1 near all known TSS (UCSC genes) were analyzed (Fig. 23A). Consistent with the above data, there was recruitment of these proteins and histone modifications to the same sites. To further validate the data from heatmap, BRD4 binding sites were classified into proximal (close to TSS) and distal (greater than 10kb away from any known TSS) sites. Aggregate plot analyses of average recruitment to proximal or distal sites for BRD4, H3K4me3 and H3K9/14ac showed increased recruitment to proximal sites, consistent with their promoter binding. Surprisingly, H2Bub1 as well as substantial fraction of BRD4 were highly recruited to the distal sites as compared to proximal sites (Fig. 23B). These observations strengthen the role of BRD4 in enhancer function and suggest a potential role for H2Bub1 in enhancer activity. To further 
determine the role of BRD4 and H2Bub1 in enhancer function, the distribution of BRD4, H3K4me3, H3K4me1 (SRR398028) (Choe et al., 2012) and H2Bub1 on distal BRD4 binding sites were also plotted (Fig. 23C). As depicted in Fig. 23B, the aggregate plots of $\mathrm{H} 2 \mathrm{Bub1}$, $\mathrm{H} 3 \mathrm{~K} 4 \mathrm{me} 1$ and $\mathrm{BRD} 4$, all indicate their presence at enhancers. However, heatmaps suggest these are different classes, marked by only H2Bub1 (transcribed region), both H3K4me3 and H3K4me1 (active genes) and absence of H3K4me3 but presence of H3K4me1, BRD4 and H2Bub1 (possible enhancer sites). Till date, H2Bub1 has been associated with transcription elongation and transcribed region but this novel function of H2Bub1 on enhancer has never been determined.

A

$\underline{\text { TSS }}$

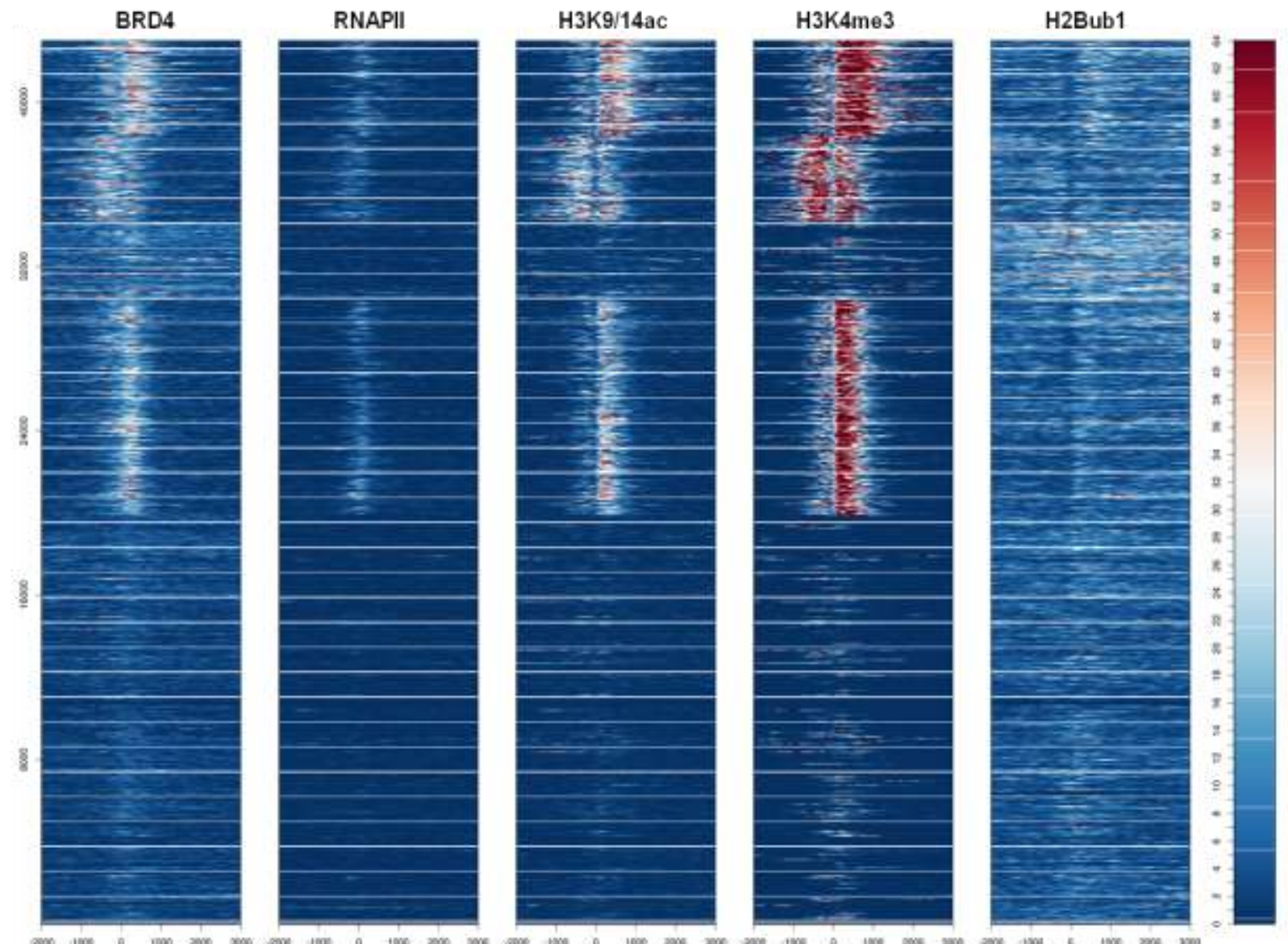



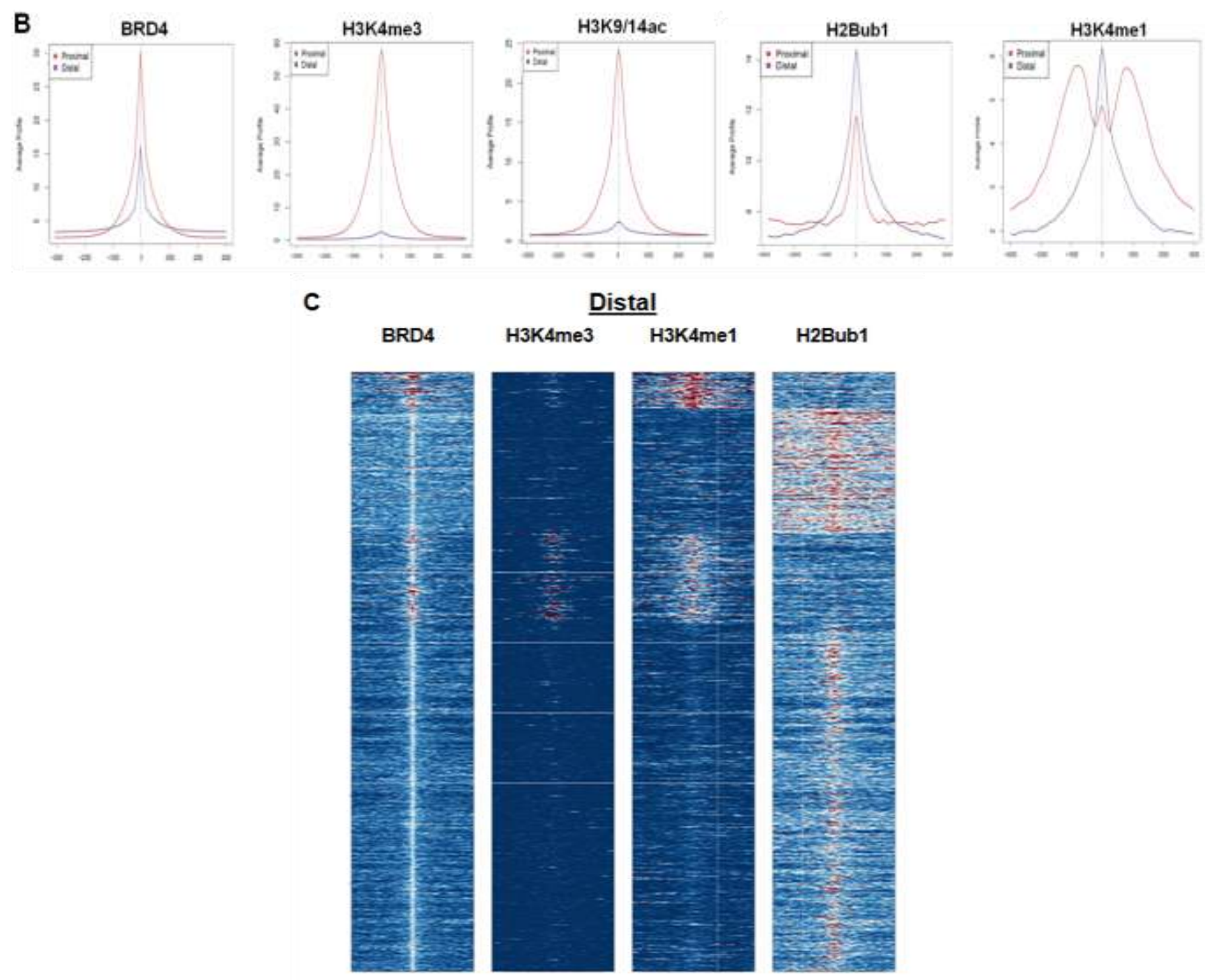

H2Bub1
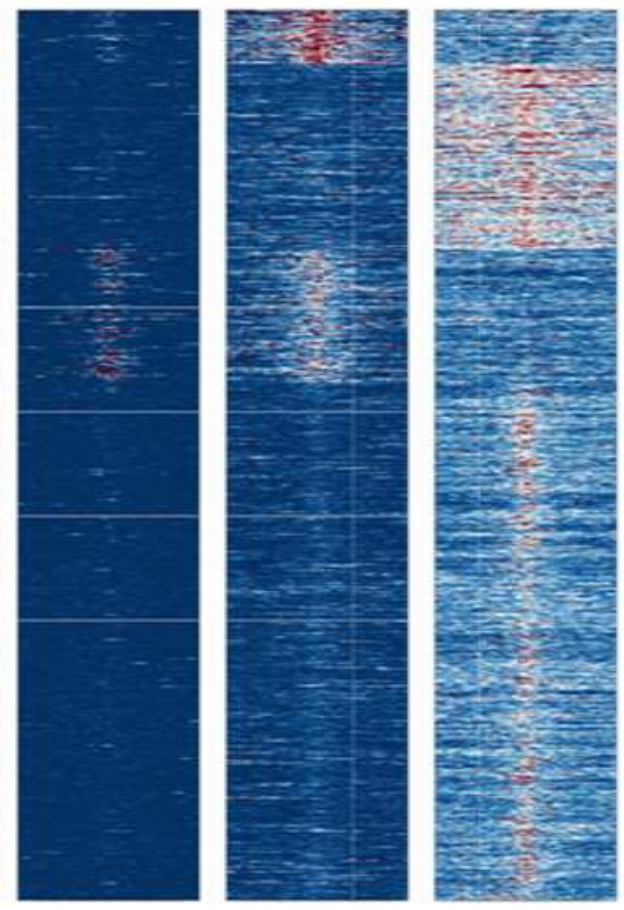

Fig. 23. Proximal and distal occupancy of BRD4 and H2Bub1. (A and C) Heatmap depicting the occupancy of BRD4 and H2Bub1 at the proximal and distal sites with $\mathrm{k}$-means clustering where $\mathrm{k}=5$. (B) Aggregate plot analysis was performed to determine the BRD4 and H2Bub1 occupancy at proximal and distal sites (greater than 10kb away from any known TSS).

\subsubsection{RNA Sequencing Reveals Regulation of Mammary Stem Cell Gene}

\section{Signature}

Extending further from the differential gene expression, gene ontology and functional gene analyses were performed. Using DAVID, a web based Gene ontology application, several genes annotated to biological pathways regulating cell migration, 
adhesion and motility were found upon RNF20, RNF40 and BRD4 depletion or following BRD4 inhibition by JQ1 (Fig. 24A). Moreover, the Gene Set Enrichment Analysis (GSEA) for the comparison of siCont versus all knockdowns was performed with Molecular signature database C2 Curated gene sets. It revealed an enrichment of gene signatures associated with a mammary stem cell phenotype (Lim et al., 2010); (Pece et al., 2010) (Cromer et al., 2004) and EMT pathways (Gotzmann et al., 2006); (Jechlinger et al., 2003) (Jaeger et al., 2007)(Sarrió et al., 2008) upon siRNF20, siRNF40, siBRD4 and JQ1, as depicted in Fig. 24B. The gene expression profile for Mammary stem cell phenotype obtained from GSEA is shown in Fig. 24C.

A

\begin{tabular}{|c|c|c|c|}
\hline Cotegory & Term & Kisist & FDR \\
\hline GOTERM_BP_FAT & G0:0006928-cell motion & 7.83 & 40.01 \\
\hline KEGG, PATHWAY & Hse04512 ECQN-ecestor interastion & 3.61 & 80001 \\
\hline SP_PIR_kEWWOROS & coll adhesian & 6.32 & 0.05 \\
\hline GOTERM_BP_FAT & 600016477-cell migration & 5.12 & 0.22 \\
\hline KEG5_PATHWAY & hsa05200-9athweps in cancer & 5.42 & 0.84 \\
\hline GOTERM_BP_FAT & 600043870-cell motility & 5.42 & 0.24 \\
\hline GOTERM_BP_FAT & 60:0031589-celloubatrate adhesion & 3.3 & 0.05 \\
\hline GOTERM_BP_FAT & 600001525-angiogenesic & 3.61 & 0.3 \\
\hline GOTERM_BP_FAT & 60:0030855-epitheial cell diterentivition & 4.22 & 20.01 \\
\hline KEGG PATHWAY & hsa05222.5malloel lung cancer & 3.61 & $\$ 0.001$ \\
\hline
\end{tabular}

B

\begin{tabular}{|c|c|c|c|c|}
\hline Gene Set Name & $\begin{array}{l}\text { SIRNF20 } \\
\text { NES }\end{array}$ & $\begin{array}{c}\text { SiRNF40 } \\
\text { NES }\end{array}$ & $\begin{array}{l}\text { siBRD4 } \\
\text { NES }\end{array}$ & $\begin{array}{l}\text { JQ1 } \\
\text { NES }\end{array}$ \\
\hline UM_MAMMARY_STEM_CEL_UP & 2.669214 & 2.6737285 & 2.34563 & 1.5145473 \\
\hline CROMER_TUMORIGENESIS_UP & 2.552813 & 2.5889904 & 2.3322124 & 1.5536558 \\
\hline PECE_MAMMARY_STEM_CELL_UP & 1.991437 & 1.5460906 & 1.3266135 & 1.7256552 \\
\hline $\begin{array}{l}\text { GOTZMANN_EPITHELIAL_TO_ } \\
\text { MESENCHYMAL_TRANSITION_UP }\end{array}$ & 1.830831 & 1.5405318 & 1.9655154 & 1.6666821 \\
\hline IAEGER_METASTASIS_UP & 1.574489 & 1.5398923 & 1.2932582 & 1.6448592 \\
\hline $\begin{array}{l}\text { JECHUINGER_EPITHEUAL_TO_ } \\
\text { MESENCHYMAL_TRANSITON_UP }\end{array}$ & 1.533922 & 1.8079038 & 1.4890907 & 1.4683328 \\
\hline UM_MAMMARY_STEM_CEL_DN & 2.316742 & 1.982711 & 1,429627 & - \\
\hline SMID_BREAST_CANCER_BASAL_DN & 1.856327 & 1.597556 & 1.254441 & . \\
\hline $\begin{array}{l}\text { SARRIO_EPTHELIAL_ } \\
\text { MESENCHYMAL_TRAANSITION_DN }\end{array}$ & 1.432256 & 1.573119 & 1.785646 & 1.364273 \\
\hline IAEGER_METASTASIS_DN & 1.428366 & - & 2.507704 & 2.7156463 \\
\hline
\end{tabular}


C

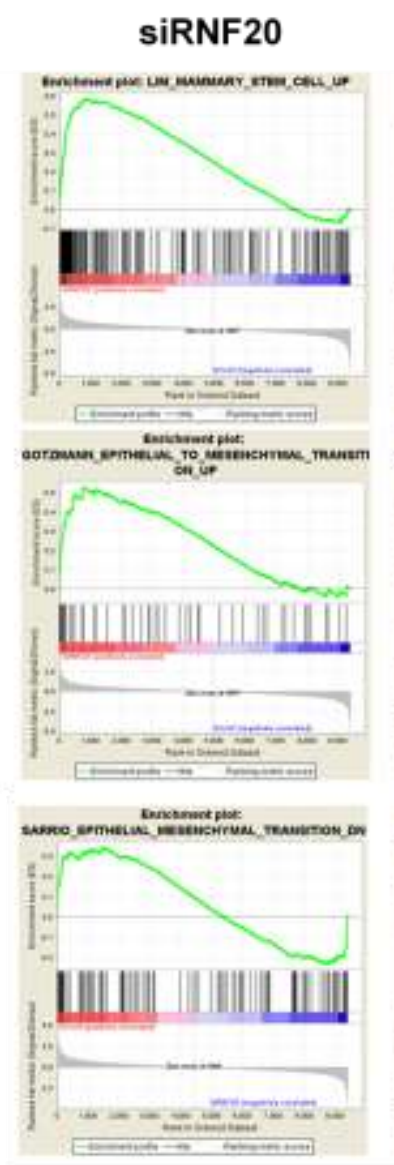

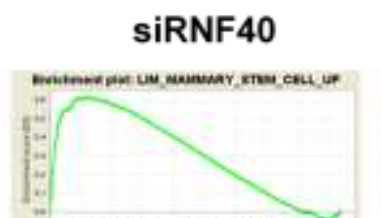
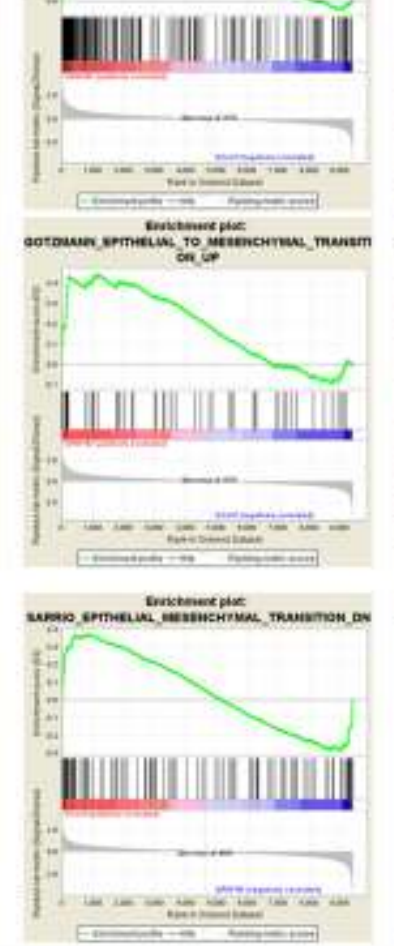
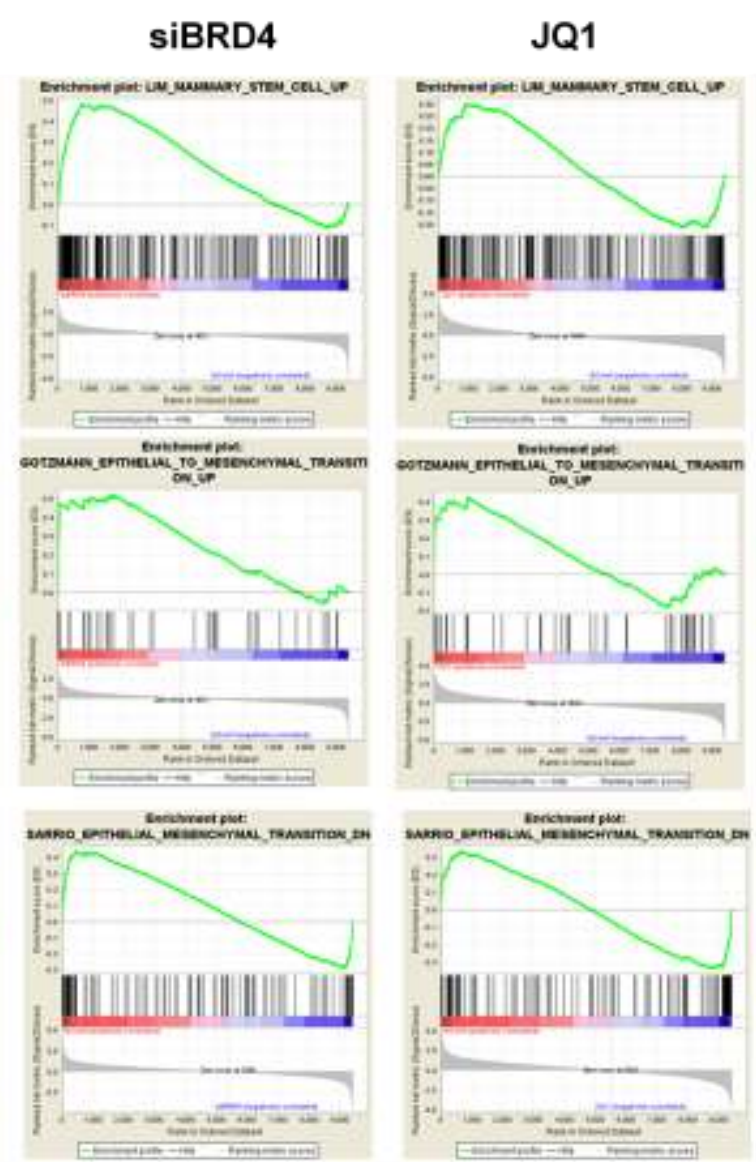

Fig. 24. BRD4 and H2Bub1 regulate mammary stem cell gene signature. (A) Gene Ontology analysis using DAVID reveals regulation of various pathways involved in cell migration and invasion. ( $B$ and $C$ ) GSEA analysis identifies mammary stem cell and EMT genes regulated upon loss of BRD4 and H2Bub1.

The mammosphere culture in breast cancer has been widely used in order to identify the enrichment of stem cell populations in vitro (Grimshaw et al., 2008). The cells transfected with control, RNF20, RNF40 or BRD4 siRNAs or treated with JQ1 were seeded as single cells into low attachment plates and allowed to grow in nonadherent and non-differentiating conditions. Consequently, cells possessing stem cell characteristics could give rise to $3 \mathrm{D}$ spheres originating from single cells. The mammospheres formed were counted and it was found that loss of RNF20, RNF40, 
BRD4 or JQ1 treatment similarly upregulated the mammosphere formation (Fig. 25A and B).

As a result, it could be interpreted that BRD4 and H2Bub1 play an important role in suppression of mammary stem cell phenotype.
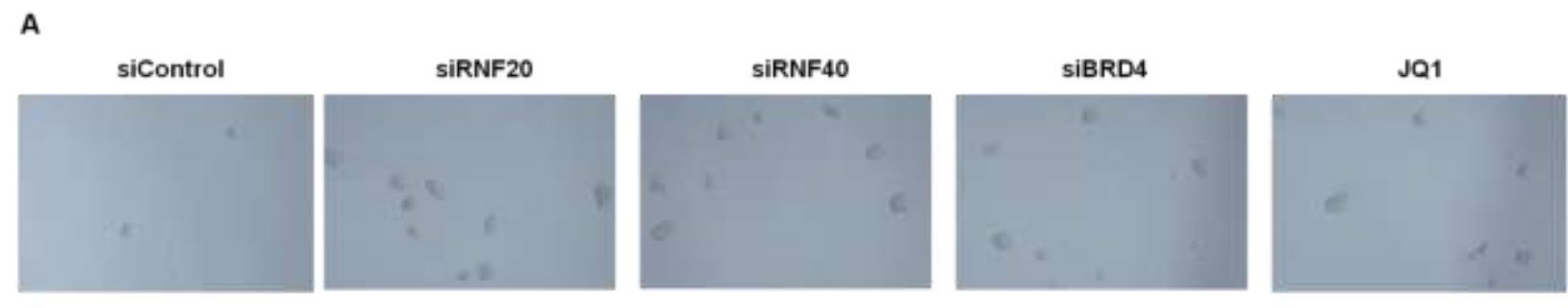

B

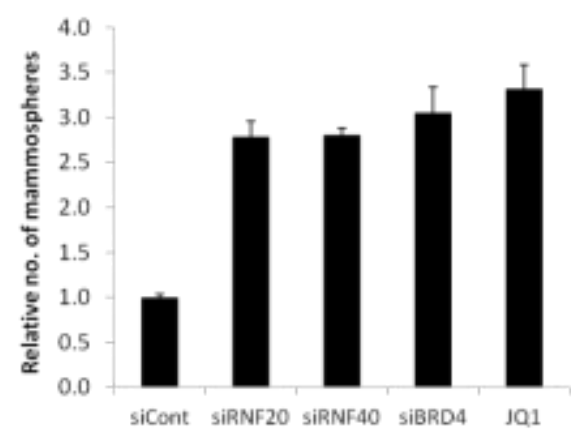

Fig. 25. BRD4 and H2Bub1 suppress mammary stem cell phenotype in MCF10A. (A) Mammosphere images upon knockdown of RNF20, RNF40 or BRD4 and JQ1 treatment. The images were taken with the microscope at 5x magnification. (B) Quantification of mammospheres formed showed increase with the knockdowns or treatment. The values were normalized to the control and represented "relative to the control"; mean values, $n=3$.

Moreover, in the RNA-seq analysis, CD24, an adhesion molecule and characteristic marker of breast cells was downregulated upon H2Bub1 depletion. Loss of CD24 is an important characteristic of stem cell-like phenotype in breast cancer cells (Al-Hajj et al. 2003; (Ponti et al., 2005). To further confirm the regulation of CD24, H2Bub1 was depleted using RNF40 knockdown and the effects were observed on CD24 protein levels and expression (Fig. 26A and B). ChIP-seq analysis of H2Bub1 indicated presence of H2Bub1 in CD24 gene body (Fig. 26C). For further 
confirmation, ChIP for H2Bub1 was performed upon RNF40 depletion. A decrease in H2Bub1 levels in CD24 transcribed region was observed indicating a direct regulation of CD24 transcription by H2Bub1 (Fig. 26D). RNF40 dependent gene, GAPDH was used as a positive control.

A

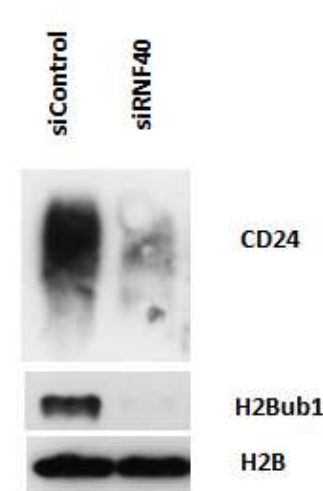

C

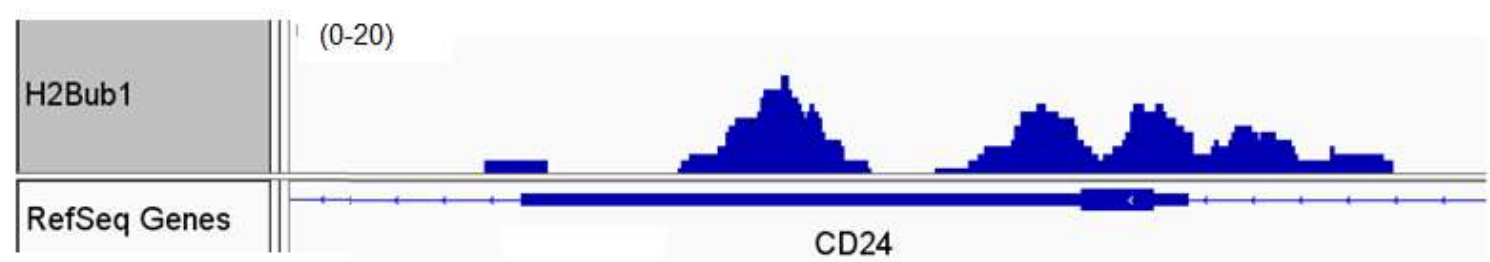

D

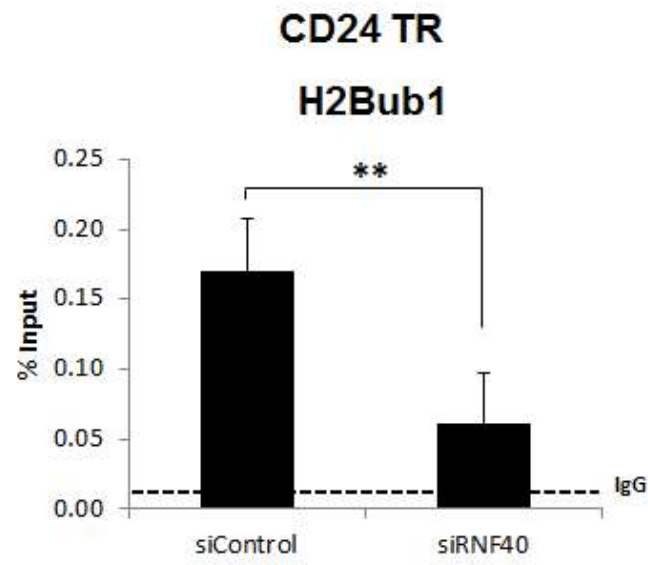

B

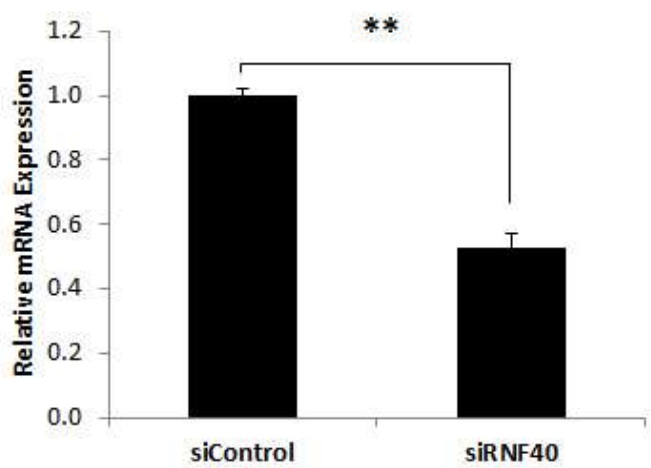

CD24
CD24

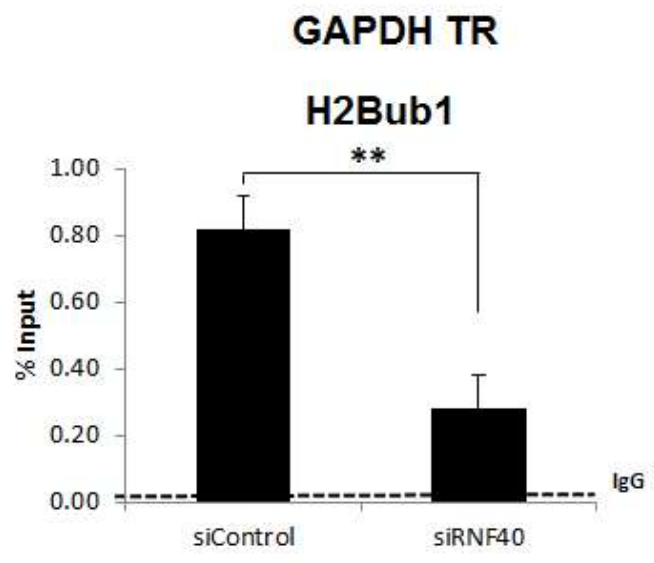

Fig. 26. H2Bub1 directly regulates CD24 expression. (A and B) RNF40 depletion leads to decrease in CD24 protein levels and mRNA expression. The expression levels were normalized to $H N R N P K$, graphed relative to the control sample and expressed as "Relative mRNA Expression"; mean values + s.d., $n=3$. (C) ChIP-seq profile of H2Bub1 indiactes its presence in CD24 gene body. (D) H2Bub1 levels decrease in CD24 TR upon RNF40 knockdown. GAPDH was taken as positive control for the presence of H2Bub1. ChIP samples were normalized to input samples and expressed as "\% Input"; mean values + s.d., $n=3$. The dotted line indicates the background binding as measured by the average signal of non-specific $\lg G$ binding across all samples and sites. For statistical significance, ANOVA test was performed and indicated by ${ }^{* *}(p \leq 0.01)$. 


\subsubsection{Loss of BRD4 and H2Bub1 is coupled with EMT phenotype}

In cancer cells, acquisition of EMT phenotype is often the first step for migration and helps transform differentiated cells into a more stem-cell like state (Mani et al., 2008); (Polyak and Weinberg, 2009). MCF10A normal mammary epithelial cells are often used as a model system to study the transition from loss of epithelial state characterized by markers like e-cadherin and cytokeratin $8 / 18$ to acquisition of mesenchymal markers like vimentin and fibronectin. After identifying EMT and mammary stem cell signatures as being specifically enriched in the RNA-seq data from knockdowns of RNF20, RNF40 and BRD4 in MCF10A, several genes involved in EMT were selected from the RNA-seq analysis and confirmed by qPCR. The graphs from the qPCR data as well as heatmap representation of gene expression are shown (Fig. 27A and B).

To further confirm the role of RNF20, RNF40 and BRD4 depletion or JQ1 treatment in suppressing EMT in MCF10A cells, the changes in EMT markers was analyzed by western blot following their depletion or inhibition by JQ1. It was observed that there was downregulation of epithelial marker, e-cadherin and upregulation of mesenchymal marker, vimentin (Fig. 28A). Further changes in markers was visualized by immunostaining for CK8/18 and vimentin in the cells transfected with siRNAs for control, RNF20, RNF40 or BRD4 or treated with JQ1 for 3 days. There was a downregulation of the epithelial marker CK8/18 and upregulation of the mesenchymal marker vimentin in cells depleted of RNF20, RNF40 or BRD4 or treated with JQ1 (Fig. 28B). 


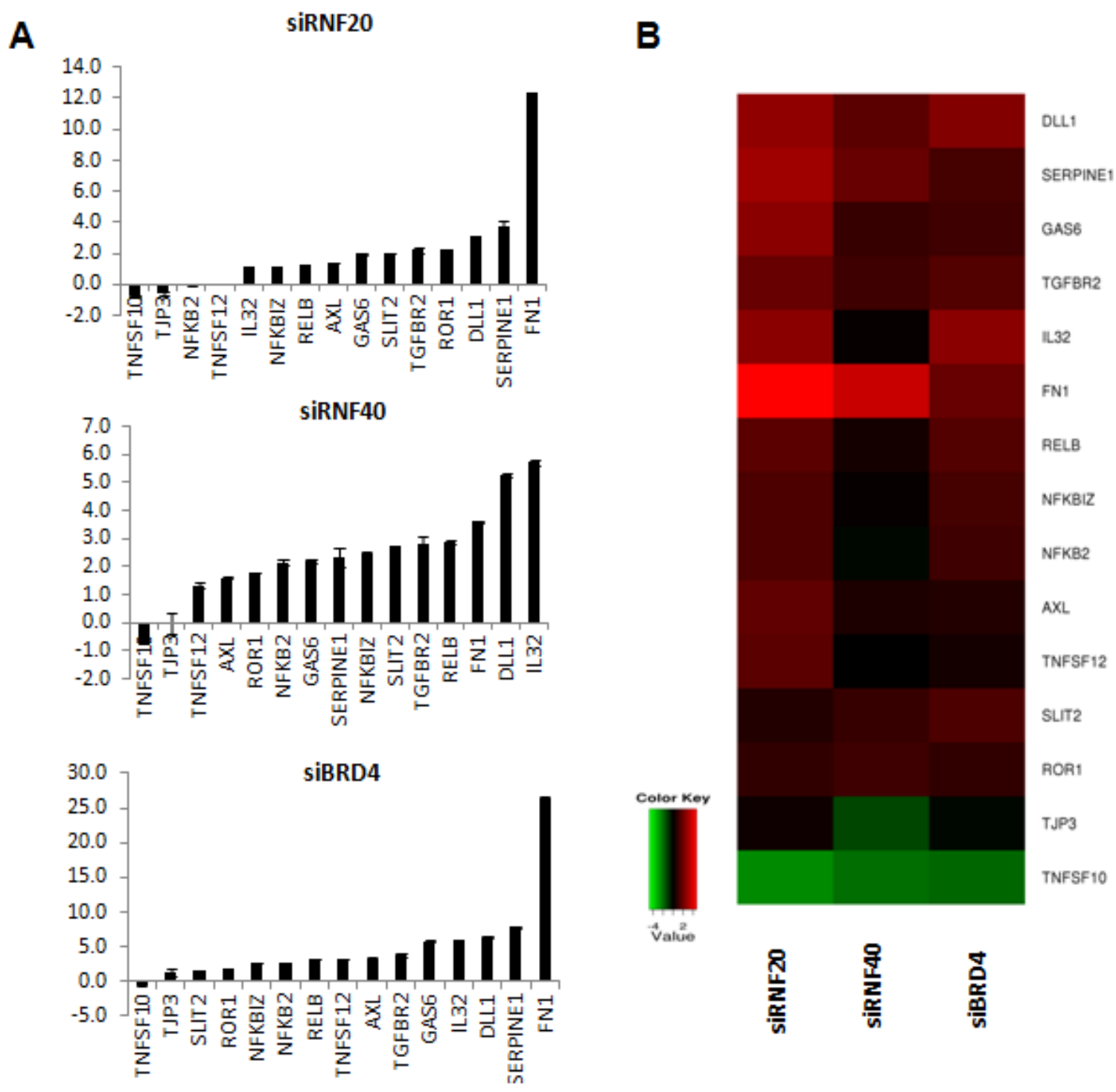

Fig. 27. BRD4 and H2Bub1 regulate the EMT target genes. (A) EMT genes regulated by BRD4 and H2Bub1 in RNA seq were confirmed by qPCR. (B) The fold regulation of these genes obtained in qPCR was represented as a heatmap. The expression levels were normalized to HNRNPK, graphed relative to the control sample and expressed as "Relative mRNA Expression"; mean values + s.d., $n$ $=3$.

An EMT phenotype often imparts the cells with a more invasive phenotype thereby making them more migratory. This could be determined by transwell migration assay where cells are allowed to migrate through $8 \mu \mathrm{m}$ membrane pores. The cells transfected with control, RNF20, RNF40 or BRD4 siRNAs or treated with JQ1 were allowed to migrate through the membrane before staining with crystal violet. It was 
observed that cells depleted of RNF20, RNF40 or BRD4 or JQ1 treated had enhanced migration, an important characteristic of EMT (Fig. 28C).

A
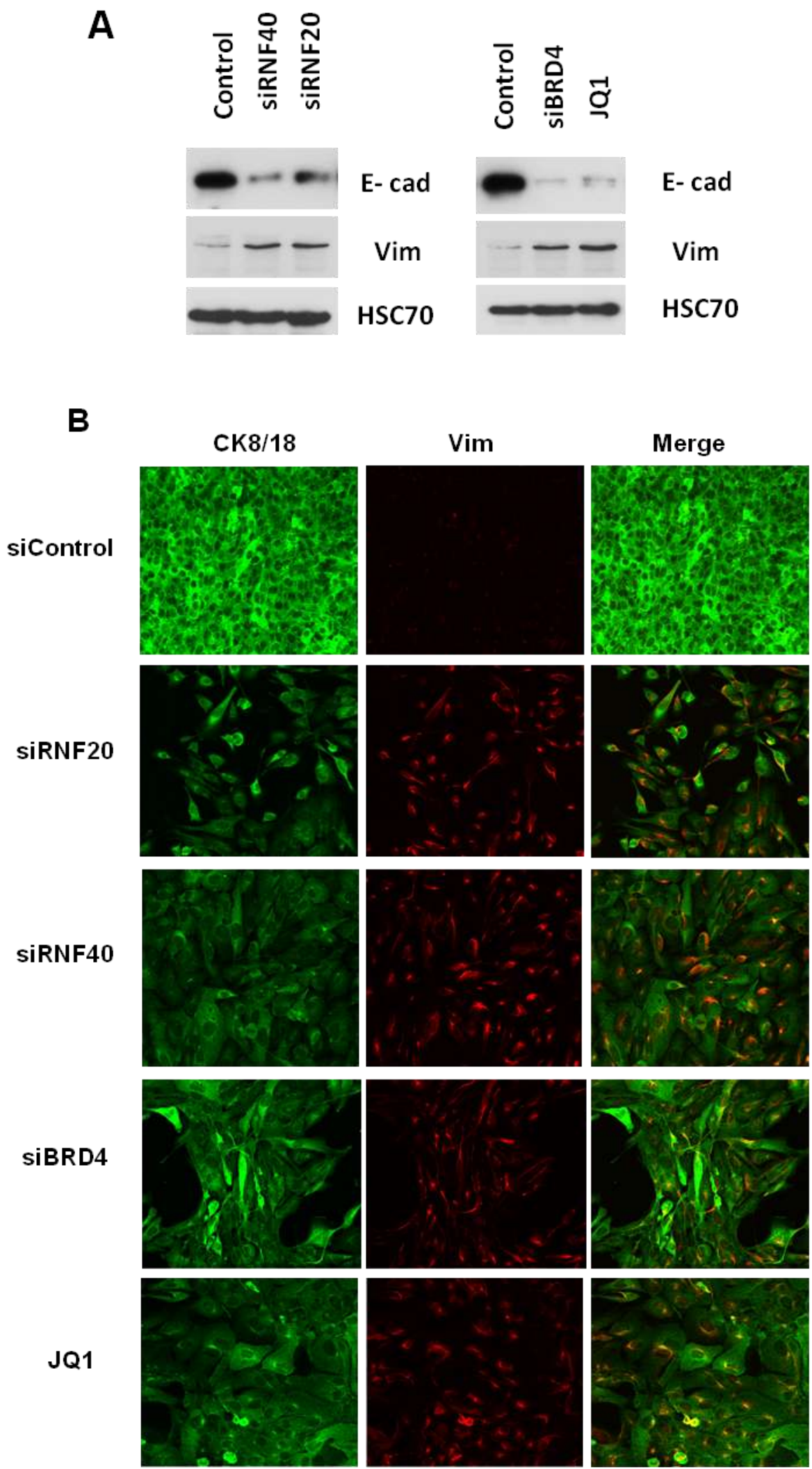
c
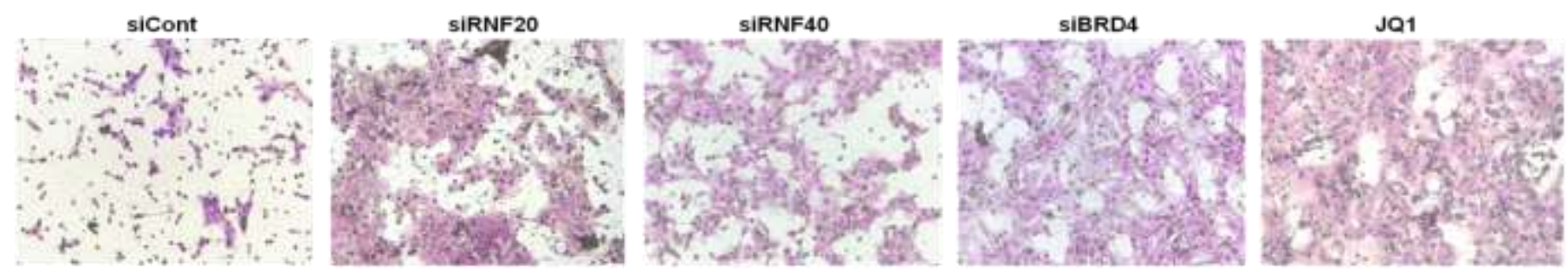

Fig. 28. Loss of BRD4 and H2Bub1 induce EMT phenotype in MCF10A cells. (A) Knockdown of RNF20, RNF40 or BRD4 and JQ1 treatment results in decrease in epithelial marker, e-cadherin and increase in mesenchymal marker, vimentin. (B) Immunoflourescence staining of CK8/18 and vimentin in these cells shows induction of EMT. (C) Transwell migration assay indicates increase in migration upon BRD4 and H2Bub1 loss.

Consistent with the effects of RNF40 depletion on CD24 levels, we next wanted to investigate if CD24 knockdown also results in EMT phenotype. Indeed, CD24 depletion using siRNA mediated knockdown resulted in acquisition of EMT phenotype (Fig. 29).

A

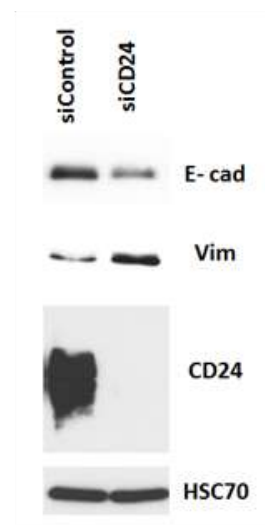

C

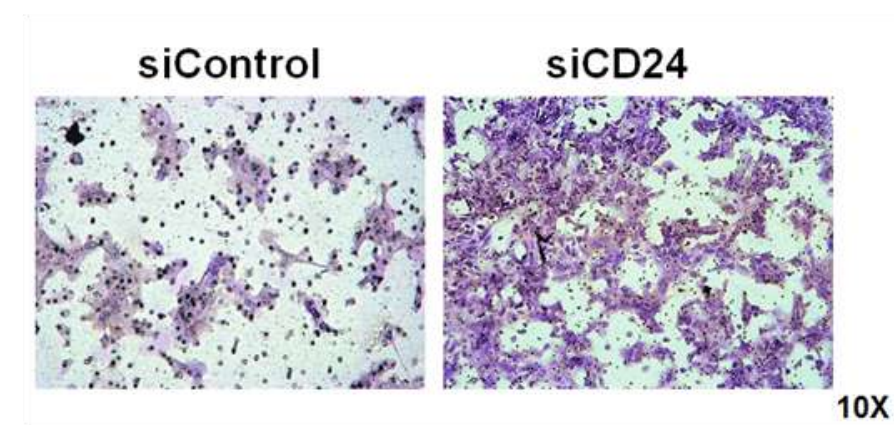

B

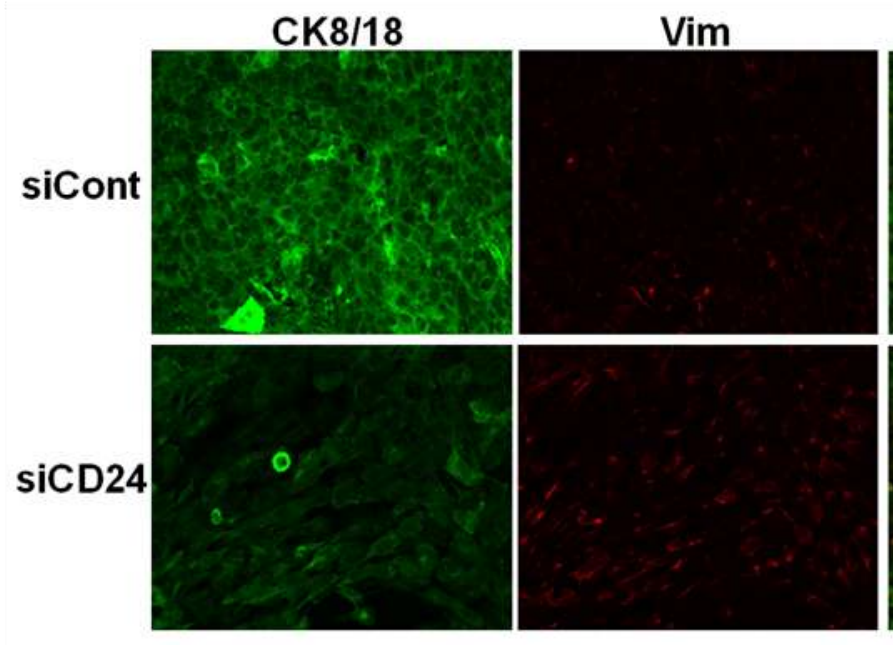

Merge
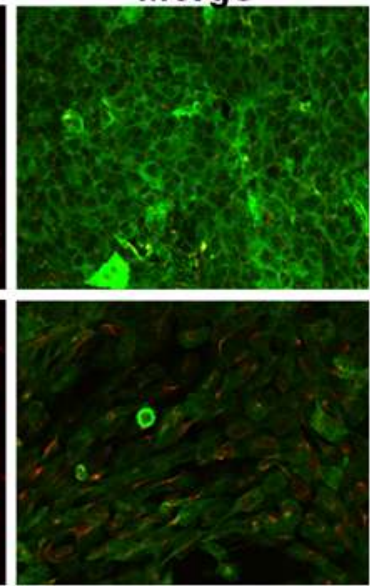
Fig. 29. CD24 depletion induces EMT in MCF10A cells. (A) siRNA mediated knockdown of CD24 leads to increase in vimentin and decrease in E-cadherin protein levels. (B) Immunoflorescence staining for CK8/18 and vimentin indicates EMT. (C) Transwell migration assay upon CD24 depletion shows enhanced migration.

\subsubsection{Conditional RNF40 knock-out mice indicates enhanced mammary gland branching}

In order to examine the in vivo regulation of RNF40 on mammary gland development, conditional mammary gland-specific RNF40 KO mice were generated. The mice engineered with RNF40 construct flanked by LoxP on both ends were crossed to mice expressing Cre recombinase under the control of the mouse mammary tumor virus promoter (MMTV-Cre) to get targeted knock-out of RNF40 in the mammary gland. Semi-quantitative PCR analysis revealed decrease in RNF40 levels in MMTV-Cre-RNF40 ${ }^{\text {loxP/loxP }}$ as compared to littermate controls (Fig. 30).

\begin{tabular}{|c|c|c|}
\hline Mice & RNF40 & MMTV-Cre \\
\hline 107 & $+/+$ & - \\
\hline 109 & $+/+$ & + \\
\hline 114 & $+/+$ & + \\
\hline 115 & $+/-$ & * \\
\hline 140 & $+/+$ & - \\
\hline 143 & $+/+$ & + \\
\hline
\end{tabular}

RNF40 - LoxP

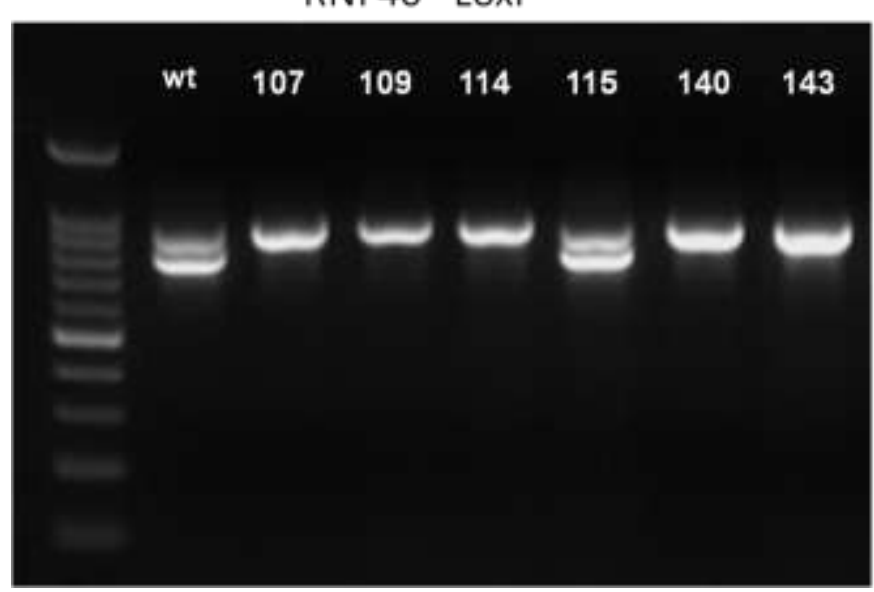

MMTV-Cre

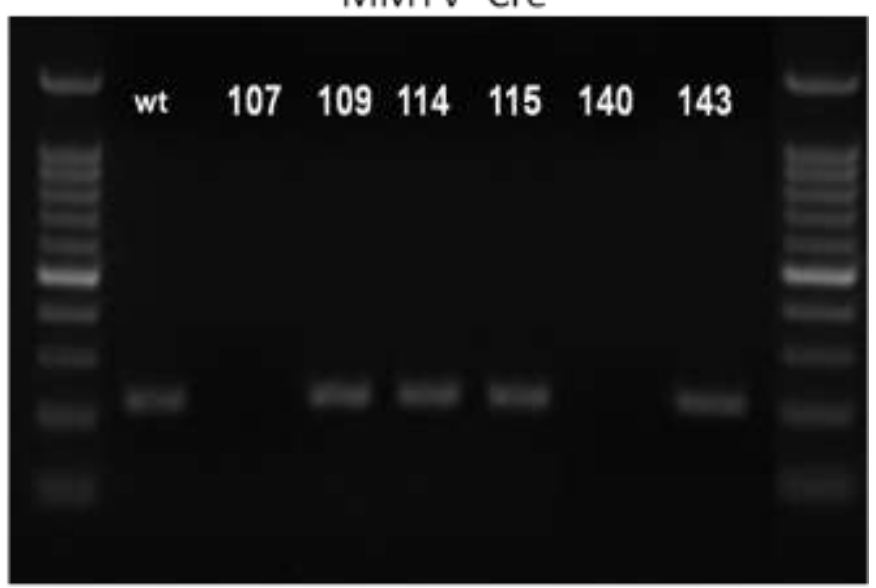

Fig. 30. Confirmation of RNF40 knockout in the mammary glands. The genotype of the mice was confirmed by semi-quantitative PCR for RNF40 and MMTV-Cre. 
The 6-week old mice were dissected and the mammary gland phenotype was observed. Whole mount images with carmine alum stained mammary ducts are shown in Fig. 31A. The mammary branching density was also observed at a higher magnification (Fig. 31B). The mammary ducts were counted and the branching density (no. of branches/mammary gland) between littermates was evaluated (Fig. 31C). This preliminary data indicates that there could be a phenotype of increased mammary branching between 3 independent groups of littermates. This observation needs further validation since the $n$-value ( $n=3$ each) is small. This initial data is already a huge finding and substantiates the hypothesis in vivo.

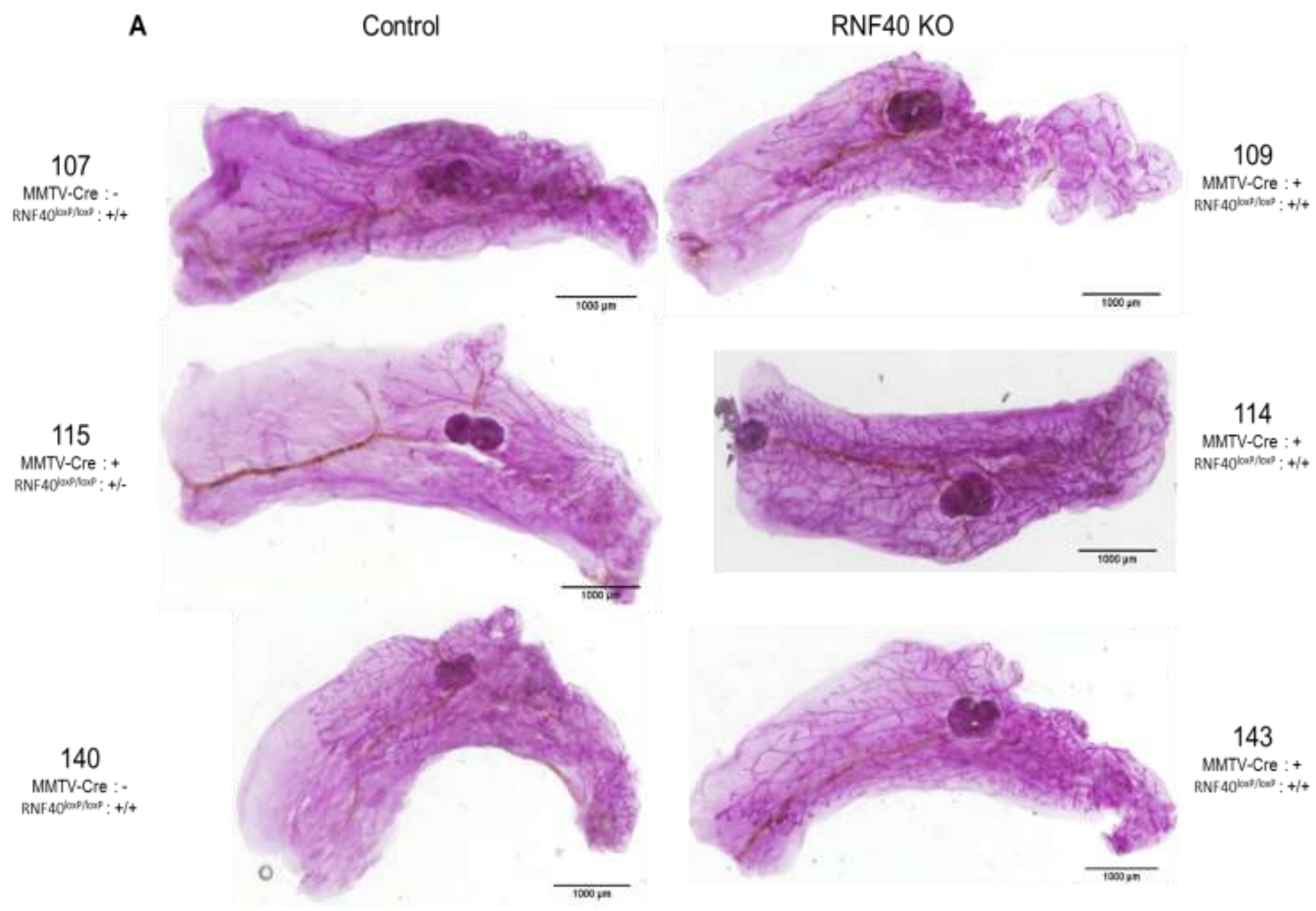




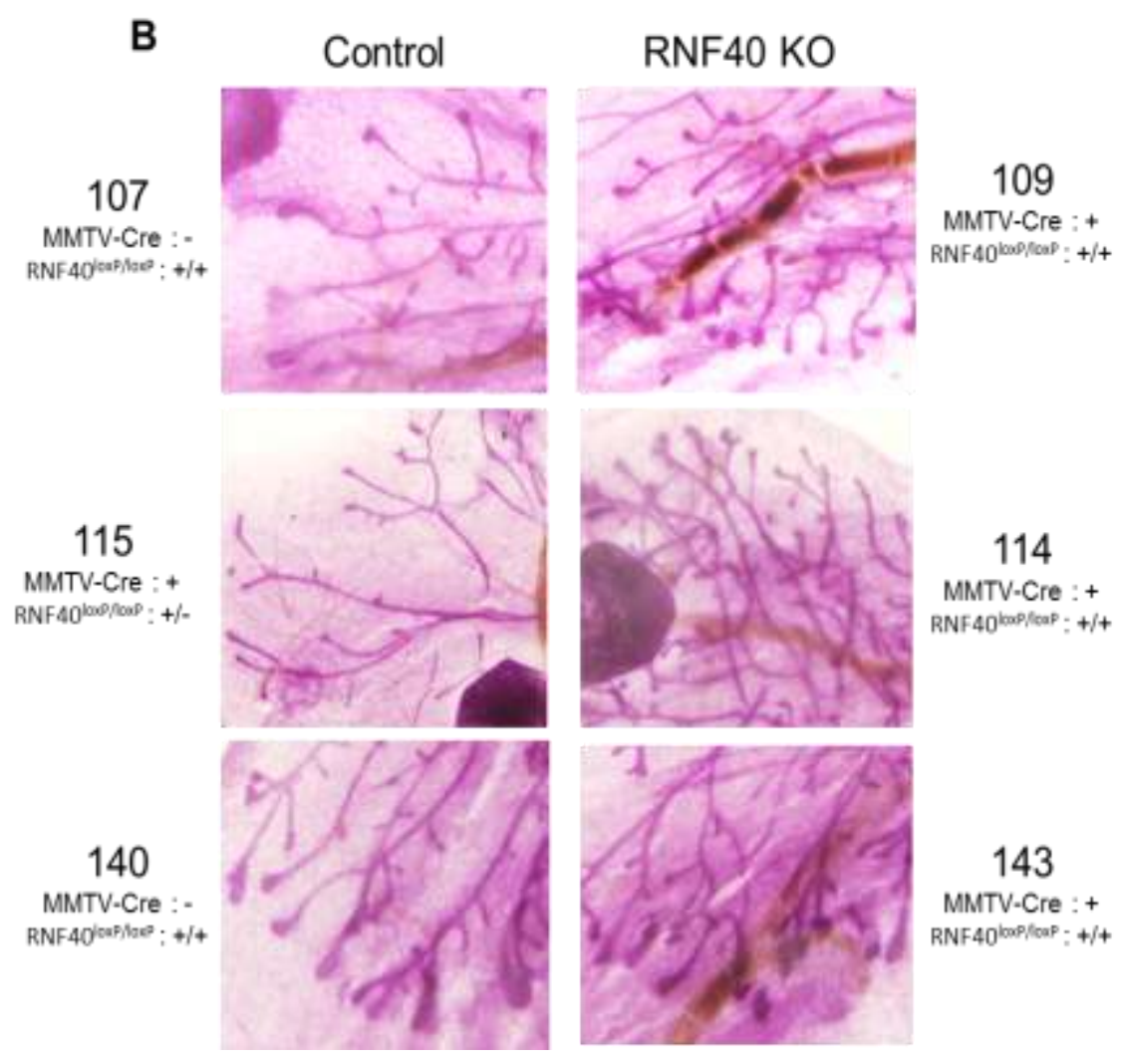

\begin{tabular}{|c|c|c|}
\hline & Control & RNF40 KO \\
\hline $107 / 109$ & 1.73 & 3.18 \\
\hline $115 / 114$ & 1.55 & 4.61 \\
\hline $140 / 143$ & 4.59 & 5.38 \\
\hline
\end{tabular}

Fig. 31. Conditional RNF40 KO mice show increased branching of mammary ducts. (A) The representative images of the mammary glands from the control and conditional RNF40 KO mice. (B) The terminal buds branching observed at a higher magnification. (C) The branching density was determined by dividing no. of branches by the mammary gland. The branches were counted and area was determined using ImageJ. 


\section{Discussion}

Transcription is a complex process regulated by the co-ordinated action of several proteins and mediators. It involves recruitment of transcription factors and histone modifications at target genes coupled with chromatin reorganization ( $\mathrm{Li}$ et al., 2007b). One important histone modification known to regulate chromatin structure as well as transcription elongation is histone H2B monoubiquitination (H2Bub1). Due to its large ubiquitin moiety, H2Bub1 plays an essential function in opening the chromatin and facilitating the recruitment of several transcription factors (Fierz et al., 2011b). It is known to be regulated by the well-established CDK9-WAC-RNF20/40 axis (Johnsen 2012; (Pirngruber et al., 2009a); Karpiuk et al. 2012). It has also been associated with the transcribed regions of genes thereby regulating transcription elongation (Minsky et al., 2008b). It is also critical for several cellular processes. It is fascinating to study and explore more of the functions associated with this modification.

\subsection{Histone chaperone SUPT6H interacts with H2Bub1 for active transcription}

SUPT6H was previously shown to bind to the Ser2-phosphorylated RNAPII CTD and promote transcriptional elongation. We have shown here that SUPT6H is required for ERa target gene expression and that it promotes the recruitment of ERa to estrogen target genes. Consistently, SUPT6H is also present in a complex with ERa and likely functions as a co-activator to promote estrogen-induced changes in chromatin structure.

Due to its direct interactions with histones $\mathrm{H} 3$ and $\mathrm{H} 4$, SUPT6H appears to be important for nucleosome disassembly and reassembly in genomic regions undergoing extensive chromatin re-organization such as actively transcribed genes. 
Based on our data from micrococcal nuclease digestion assays, SUPT6H appears to control chromatin organization over a substantial portion of the genome since its depletion leads to a more open global chromatin. Thus, we hypothesize that SUPT6H regulates transcription, at least in part by maintaining proper chromatin structural organization, possibly by preventing the improper exposure of regulatory sequences or promoter regions.

CDK9-mediated Ser2 phosphorylation of the RNAPII CTD is important for RNF20/40 recruitment to chromatin by the adaptor protein, WAC which then is required for $\mathrm{H} 2 \mathrm{~B}$ monoubiquitination (Pirngruber et al., 2009a) Zhang and Yu 2011; (Pirngruber et al., 2009b). H2Bub1 has been linked with transcription elongation based in part on its presence in the transcribed regions of active genes (Minsky et al., 2008b). Since SUPT6H also interacts with P-Ser2 RNAPII, it is possible that the WAC/RNF20/40 complex and SUPT6H travel together with the elongating RNAPII. This connection between SUPT6H and H2Bub1 had never been reported before. Consistently, our co-immunoprecipitation experiments demonstrated for the first time that SUPT6H and RNF40 are present in a complex and may co-regulate target gene expression in part through H2Bub1. The effect of SUPT6H depletion on H2Bub1 and RNF40 protein levels further supports that SUPT6H regulates this important histone modification.

We previously demonstrated that H2Bub1 levels are inversely correlated with tumor malignancy in breast cancer (Prenzel et al., 2011) but no studies had investigated the role of histone chaperone, SUPT6H in tumorigenesis. On further examining the online Oncomine database, a correlation between SUPT6H expression and 
malignancy was observed. These studies are consistent with our findings that both SUPT6H and H2Bub1 levels decrease with decreased tumor differentiation status. Consistent with a role of SUPT6H and H2Bub1 in maintaining a differentiated phenotype, we now show that SUPT6H, like H2Bub1 (Karpiuk et al., 2012a), is required for maintaining a luminal mammary epithelial phenotype and for multipotent stem cell differentiation. Consistently, we observed a strong correlation between SUPT6H and RNF40 mRNA levels in both hMSCs and adipose tissue. Thus, it is likely that SUPT6H and H2Bub1 act in the same pathway to promote estrogenresponsive and lineage-specific gene transcription.

During myoblast differentiation, SUPT6H was recently found to interact with the two important histone demethylases, KDM6A/UTX and KDM6B/JMJD3 which function to remove Polycomb-mediated repressive marks, H3K27me3 on target genes (Chen et al. 2012; Wang et al. 2013). Consequently, SUPT6H helps to remove gene repression and enables active transcription by interaction with elongating RNAPII. We suggest a model for the role of SUPT6H in transcription in which the target gene requires SUPT6H in order to suppress H3K27me3-mediated repression. In this model, SUPT6H is recruited to estrogen-regulated and lineage-specific genes and functions to further recruit additional interaction partners including the H3K27 demethylases KDM6A/UTX and KDM6B/JMJD3 and the H2B ubiquitin ligase complex RNF20/40 to the elongating RNAPII (Fig. 32). This leads to a suppression of $\mathrm{H} 3 \mathrm{~K} 27$ me3 and the maintenance of $\mathrm{H} 2 \mathrm{Bub} 1$ to sustain an active epigenomic status. 


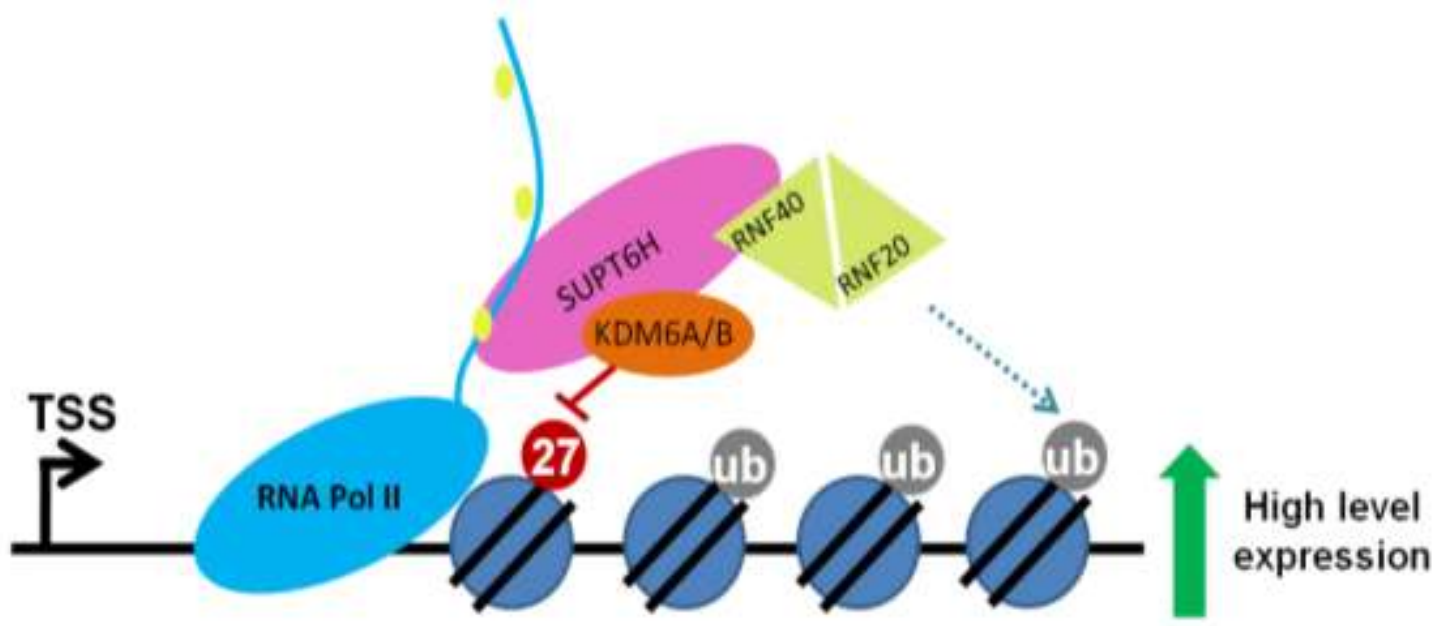

\section{Active Transcription}

Fig. 32. Model illustrating the role of SUPT6H in transcription. Red circles marked "27" depict H3K27me3 and gray circles with "ub" denote H2Bub1. During transcription, SUPT6H regulates $\mathrm{H} 3 \mathrm{~K} 27 \mathrm{me} 3$ and H2Bub1 on the chromatin via interactions with several factors.

The results presented here are consistent with our findings in primary breast cancer and suggest that decreased SUPT6H expression or inactivation of the H2B monoubiquitination regulatory pathway may provide an alternative mechanism which facilitates Polycomb-mediated repression during malignant progression. Whether and how the SUPT6H-RNF40-H2Bub1 pathway can be harnessed to regulate estrogen receptor-regulated transcription in breast cancer or to suppress differentiation of stem cells for regenerative medicine purposes warrants further investigation.

\subsection{BRD4 and H2Bub1 act together during transcription}

BRD4 is known to bind highly acetylated histones, $\mathrm{H} 3$ and $\mathrm{H} 4$ (Wu and Chiang, 2007b) and recruits P-TEFb to genes for active transcription (Jang et al. 2005; (Yang et al., 2005). The regulation of H2Bub1 via CDK9-WAC-RNF20/40 pathway is also 
known. The next question we addressed was the link between BRD4 and H2Bub1 during transcription. Our data points out that there is a decrease in H2Bub1 levels upon loss of BRD4. This connection was further strengthened by the RNA-seq analysis which showed a large overlap in the number of genes regulated by BRD4 and H2Bub1. In fact, the pattern of gene regulation by BRD4 and H2Bub1 was also quite similar. ChIP-seq analysis also highlighted the recruitment of BRD4 and H2Bub1 to the same sites. Moreover, we have shown that the overall gene expression is correlated with BRD4 and H2Bub1 occupancy on those genes. These results highlight that they could be acting together via BRD4-CDK9-WAC-RNF20/40 axis.

\subsection{BRD4 and H2Bub1 "teamwork" suppresses EMT and mammary stem cell phenotype}

We have shown in this study that upon loss of BRD4 and H2Bub1, mammary cells switch from an epithelial to a mesenchymal phenotype as well as acquire stem celllike phenotype. Under normal conditions, there is expression of epithelial genes while mesenchymal genes that can impart invasive properties to cells remain suppressed. In these circumstances, BRD4 and H2Bub1 could be performing their task of gene expression regulation by promoting expression of epithelial genes and tumor suppressors. However, upon BRD4 and H2Bub1 depletion, there is a switch in gene regulation where mesenchymal genes can no longer be suppressed. Based on

this, we propose a model for the mechanism of action of BRD4 and its role in suppression of EMT phenotype (Fig. 33). 
A

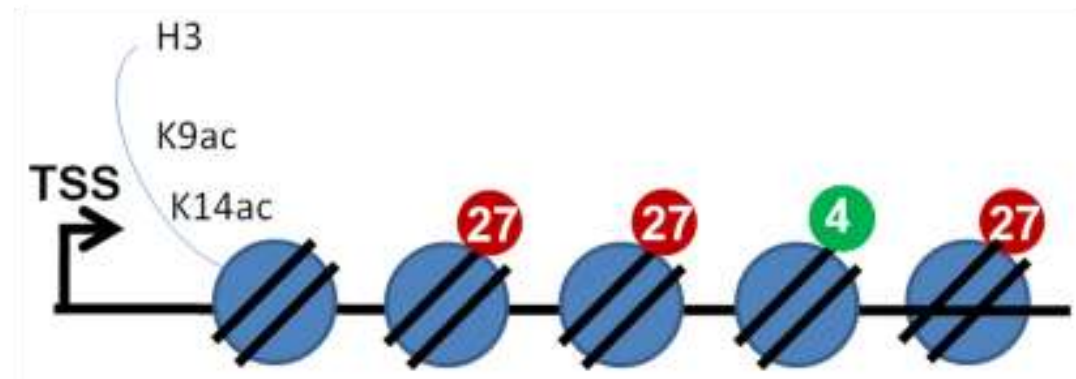

B

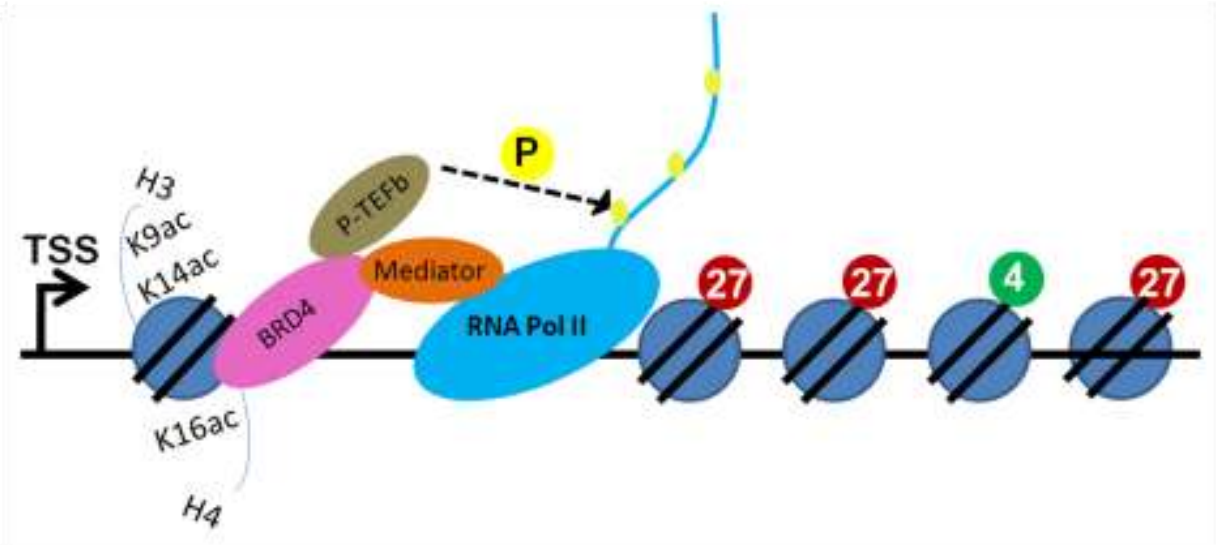

C

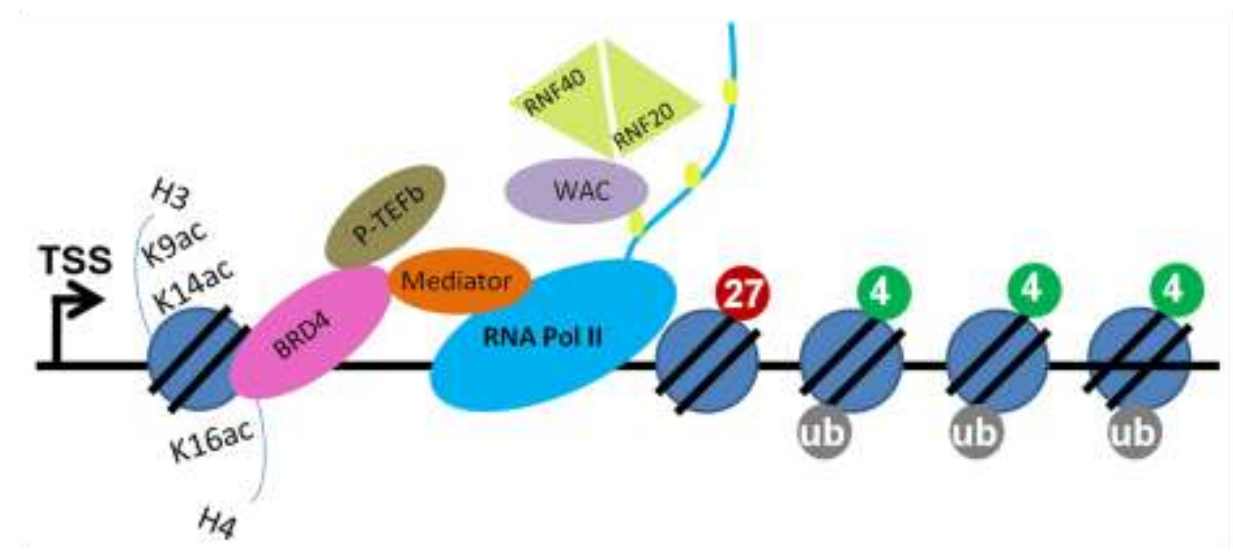

Fig. 33. Possible mechanism of H2Bub1 recruitment by BRD4 associated pathway. Red circles marked "27" depict H3K27me3, green circles marked "4" represent H3K4me3 and gray circles with "ub" denote H2Bub1. During transcription, BRD4 binds to acetylated chromatin and recruits H2Bub1 on the chromatin via CDK9-WAC-RNF20/40 axis.

In normal cells, histones surrounding the epithelial and tumor suppressor genes get acetylated by various acetyltransferases. This is then sensed by BRD4 and helps to recruit P-TEFb and RNAPII to the target genes. CDK9 mediates phosphorylation of Ser2-RNAPII and recruits adaptor protein, WAC which in turn recruits RNF20/40 
heterodimer responsible for H2Bub1. Presence of H2Bub1 in the transcribed regions indicates active transcription of genes regulating the epithelial phenotype of the cells.

\subsection{Possible mechanisms of regulation by BRD4 and H2Bub1}

Several studies in recent years have highlighted misregulation of epigenetic factors during EMT. There are several hypotheses that could explain the possible mechanism of regulation by BRD4 and H2Bub1 on EMT target genes.

\subsubsection{Gene Bivalency}

Human and mouse ES cells are enriched with functionally opposite histone modifications, H3K27me3 (a repressive mark) and H3K4me3 (an activating mark) (Azuara et al., 2006) (Bernstein et al., 2006); (Pan et al., 2007); (Zhao et al., 2007). This is referred to as a state of bivalency. H3K4me3 is deposited by proteins of Trithorax group while trimethylation of H3K27 is executed by PRC2 (Schuettengruber et al., 2007).

One such example of bivalency in EMT genes was illustrated by Wu et al. 2011. They had shown that epithelial genes are bivalent and their expression depends upon the extracellular signals. Under normal state, epithelial genes are expressed by resolution of their bivalency but upon EMT inducing conditions like hypoxia, epithelial genes are silenced and remain poised by both activating, H3K4me3 and repressive, H3K27me3 marks.

Our group had previously shown that RNF40 and H2Bub1 were important for resolution of bivalency on adipocyte-specific genes (Karpiuk et al., 2012a). On the same lines, we speculate that BRD4 could play a role in signal-dependent resolution of bivalency through H2Bub1-dependent pathway. Under normal conditions, BRD4 and H2Bub1 could promote transcription of epithelial genes by removing the repressive marks, H3K27me3 and resolving the gene bivalency. This could be 
achieved by the recruitment of demethylases. The demethylases responsible for the removal of H3K27me3 include KDM6A/UTX and KDM6B/JMJD3 (Agger et al., 2007). There are no evidences suggesting direct interaction of H2Bub1 and these demethylases. However, it is known that UTX and JMJD3 are important for transcription elongation (Seenundun et al., 2010); Chen et al. 2012). Thus, it could be possible that BRD4 interacts with these demethylases and helps in transcription. This proposed mechanism could be true since our data indicates that loss of BRD4 and H2Bub1 results in repression of epithelial genes which could be due to the failure to recruit the demethylases for removal of repressive marks and resolution of bivalency.

The role of BRD4 and H2Bub1 in resolution of bivalency on epithelial genes could be determined by comparing ChIP-seq profiles of H3K27me3 and H3K4me3 in control and BRD4 or RNF20/40 depleted cells. Moreover, the interaction partners of BRD4 and H2Bub1 could be determined by performing co-immunoprecipitation experiments followed by mass spectrometry. These experiments would be able to shed more light in this context.

It is also important to note that BRD4 and H2Bub1 are likely not responsible for resolution of bivalency of mesenchymal genes. In that case, depletion of BRD4 and H2Bub1 should result in repression of mesenchymal genes due to the inability to resolve the bivalency. However, our data indicates that loss of BRD4 and H2Bub1 rather increase the expression of mesenchymal genes. This probably indicates the repressive function of BRD4 and H2Bub1. 


\subsubsection{Repressive role of BRD4 and H2Bub1}

So far, BRD4 and H2Bub1 have been shown to positively regulate the transcription (Jang et al. 2005; (Yang et al., 2005); Minsky et al. 2008). However, there seems to be a repressive function of BRD4 and $\mathrm{H} 2 \mathrm{Bub1}$ in suppressing the expression of mesenchymal genes. As stated before, in normal cells, there is expression of epithelial genes while the mesenchymal genes are repressed and our data highlights that depletion of BRD4 and H2Bub1 results in the upregulation of mesenchymal genes and other EMT transcription factors. The only explanation could be that BRD4 and H2Bub1 keep the expression of mesenchymal genes under check by cooperating with p53 or through PRC2-mediated gene repression.

This is in concordance with the tumor suppressor role of BRD4 and H2Bub1 (Crawford et al., 2008); Shema et al. 2008). It was shown previously that RNF20 acts as a tumor suppressor by promoting transcription of p53 and repressing transcription of proto-oncogenes (Shema et al., 2008b). Loss of H2Bub1 is also coupled with tumorigenesis (Prenzel et al., 2011). BRD4 depletion has also been associated with tumorigenesis (Alsarraj et al., 2011b) and predicts patient survival (Crawford et al., 2008).

The above correlation could be confirmed by examining the p53 status and H3K27me3 levels in the BRD4 and H2Bub1 depleted cells. Moreover, ChIPsequencing of H3K27me3 would help address its repressive function on EMT target genes.

\subsubsection{Regulation of tumor suppressor microRNAs}

MicroRNAs (miRNAs) are small (22nt long) non-coding RNAs that regulate the gene expression by targeting mRNAs for degradation or suppression of translation (Bartel, 
2004). They have been recognized as important tumor suppressors by regulating the expression of the proto-oncogenes. It is also well established that miRNAs are often dysregulated in human cancers. Among several known miRNAs, miRNA-200 family is well established in regulating the EMT phenotype and stemness of cells (Wellner et al. 2009; (Korpal et al., 2008).

In our ChIP-seq data for BRD4 and H2Bub1, we found substantial BRD4 and H2Bub1 occupancy on the miRNA-200 family genes. This indicates that BRD4 and H2Bub1 could directly regulate the expression of tumor suppressor miRNAs and prevent expression of mesenchymal genes. Thus, loss of BRD4 and H2Bub1 would perturb the regulation and result in decreased expression of miRNA-200 family genes. As a result, it would upregulate the expression of several EMT transcription factors like ZEB-1 (Wellner et al., 2009b) and promote tumorigenesis.

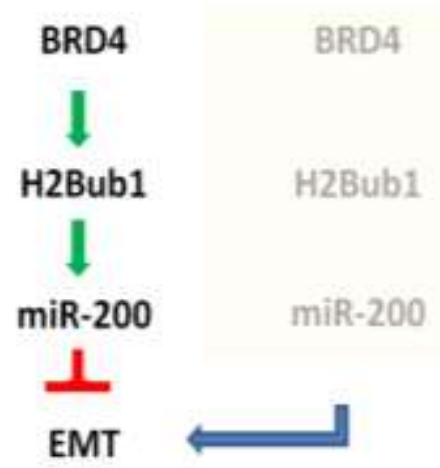

Fig. 34. Graphical representation of the role of BRD4 and H2Bub1 in miRNA regulation.

This proposed mechanism seems quite convincing and is being investigated further. The first step is to examine the expression of miRNA-200 family upon BRD4, RNF20 and RNF40 knockdowns. For this, we would like to perform high-throughput RNAsequencing of miRNA population from the knockdown samples. This would address the regulation of miRNA expression and likely provide the mechanistic basis for regulation of BRD4 and H2Bub1 in EMT and mammary stem cell phenotype. 


\subsubsection{Role of BRD4 and H2Bub1 in regulation of enhancer RNA expression}

Enhancer RNAs (eRNAs) are relatively short (50-2000 nt long) non-coding RNAs that are transcribed from the enhancer elements. Enhancer domains marked by histone modification, H3K4me1 have RNAPII binding that leads to bi-directional transcription of small eRNAs. These eRNAs seem to play an important role in transcriptional regulation of the nearby target genes either by proximal or distal regulation (Kim et al., 2010); (Ong and Corces, 2011); (Li et al., 2013). However, not much has been known about the mechanism of action of these eRNAs.

Our ChIP-seq data indicated the presence of BRD4 and H2Bub1 on distal sites marked by H3K4me1 (enhancers). This indicates that BRD4 and H2Bub1 could have a potential regulation of enhancer activity. All previous studies have identified H2Bub1 to be present in transcribed region of genes and associated with transcription elongation (Minsky et al., 2008b). However, our ChIP-seq data identifies a novel function of H2Bub1 and enhancer RNA regulation. Thus, we hypothesize that the expression of epithelial genes or tumor suppressor miRNAs are regulated by the eRNAs in the presence of BRD4 and H2Bub1. However, upon depletion of BRD4 and H2Bub1, these genes are no longer regulated by their eRNAs and are repressed.

This mechanism warrants further investigation which could be confirmed by RNAsequencing of short RNAs and then mapping them to the genome to determine their target gene regulation.

\subsubsection{Loss of H2Bub1 mimics activation of deubiquitinases (DUBs)}

The mechanism of addition of $\mathrm{H} 2 \mathrm{~B}$ monoubiquitination and its active removal by deubiquitinases is a tightly regulated process. In humans, H2Bub1 is deubiquitinated 
by the SAGA complex (Lang et al., 2011). Dysregulation of a fine balance between ubiquitination and deubiquitination could severely affect the cells. Depletion of H2Bub1 would interfere with the balance and result in accumulation of DUBs due to absence of substrates for deubiquitination.

Our data indicates that loss of H2Bub1 results in the acquisition of EMT and mammary stem cell phenotype. Overexpression of DUBs like USP22 has been found in tumors and likely results in stem cell-like phenotype and poor patient outcome (Glinsky et al., 2005); Zhang et al. 2008; Liu et al. 2011). The loss of H2Bub1 could be compared to an overexpressed state of deubiquitinases. Thus, the equilibrium between H2Bub1 and DUBs could be critical in imparting tumorigenic properties.

This could be determined by depletion of SAGA complex components and examining the effects on gene expression by RNA-seq analysis. A comparison of the gene expression profiles upon H2Bub1 and SAGA subunits depletion would help us to understand this complex mechanism.

\subsection{BRD4 inhibition by JQ1 enriches cancer stem cell pool}

A small molecule inhibitor, JQ1 was described that could bind to the acetyl lysine pocket of BET family proteins and prevent their binding to the chromatin (Filippakopoulos et al., 2010). BET protein inhibition by JQ1 proved to be an anticancer strategy in Myc-dependent myelomas (Zuber et al., 2011b). This was shown to be beneficial due to the role of BRD4 in sustaining Myc expression and disease maintenance by Myc target genes (Delmore et al., 2011). However, not all cancers are Myc-dependent.

The data presented in this study demonstrates that depletion of BRD4 or inhibition by JQ1 results in induction of mammary stem cell phenotype and enrichment of this 
stem cell population. The cancer stem cell (CSC) hypothesis has highlighted the significance of cancer cells which acquire stem cell-like and self-renewal properties (Reya et al., 2001). It has been widely accepted and emphasizes the relapse of malignancy due to the cancer stem cell population in tumors. We show here that using JQ1 as an anti-tumor drug might not be favourable and would result in enrichment of CSC population. There could be tumor regression for the initial phase of treatment but would ultimately result in relapse of tumor after the therapy is withdrawn, thereby resulting in tumors with enhanced metastatic properties which could no longer be treated by chemotherapy.

Taken together, this study reveals that $\mathrm{H} 2 \mathrm{~B}$ monoubiquitination regulates ERadependent signaling through histone chaperone, SUPT6H possibly via CDK9-WACRNF20/40 axis. Decrease in SUPT6H levels correlated with decrease in tumor differentiation status of the breast tissue sections. These data identify SUPT6H as a new epigenetic regulator of ERa activity and cellular differentiation. Moreover, this study also demonstrated that BRD4 suppresses the stem cell phenotype in mammary cells by H2Bub1 pathway. RNA-seq analysis revealed that loss of BRD4 and H2Bub1 results in the upregulation of mammary stem cell gene signature and imparts invasive properties to the cells thereby promoting tumorigenesis and metastasis. Suppression of BRD4 by JQ1 resulted in enrichment of mammary cancer stem cells which could give rise to more aggressive tumors. Altogether, restoration of BRD4 and H2Bub1 by inhibiting their degradation in breast cancer might provide useful anti-cancer therapies. 


\section{References}

Agger, K., Cloos, P.A.C., Christensen, J., Pasini, D., Rose, S., Rappsilber, J., Issaeva, I., Canaani, E., Salcini, A.E., and Helin, K. (2007). UTX and JMJD3 are histone H3K27 demethylases involved in HOX gene regulation and development. Nature 449, 731-734.

Agoulnik, I.U., Vaid, A., Bingman, W.E., Erdeme, H., Frolov, A., Smith, C.L., Ayala, G., Ittmann, M.M., and Weigel, N.L. (2005). Role of SRC-1 in the Promotion of Prostate Cancer Cell Growth and Tumor Progression. Cancer Res. 65, 7959-7967.

Alberts, B., Johnson, A., Lewis, J., Raff, M., Roberts, K., and Walter, P. (2002). Chromosomal DNA and Its Packaging in the Chromatin Fiber.

Ali, S., and Coombes, R.C. (2000). Estrogen Receptor Alpha in Human Breast Cancer: Occurrence and Significance. J. Mammary Gland Biol. Neoplasia 5, 271-281.

Ali, S., Metzger, D., Bornert, J.M., and Chambon, P. (1993). Modulation of transcriptional activation by ligand-dependent phosphorylation of the human oestrogen receptor $A / B$ region. EMBO J. 12, 1153-1160.

Alsarraj, J., Walker, R.C., Webster, J.D., Geiger, T.R., Crawford, N.P.S., Simpson, R.M., Ozato, K., and Hunter, K.W. (2011a). Deletion of the Proline-Rich Region of the Murine Metastasis Susceptibility Gene Brd4 Promotes Epithelial-to-Mesenchymal Transition- and Stem Cell-Like Conversion. Cancer Res. 71, 3121-3131.

Alsarraj, J., Walker, R.C., Webster, J.D., Geiger, T.R., Crawford, N.P.S., Simpson, R.M., Ozato, K., and Hunter, K.W. (2011b). Deletion of the Proline-Rich Region of the Murine Metastasis Susceptibility Gene Brd4 Promotes Epithelial-to-Mesenchymal Transition- and Stem Cell-Like Conversion. Cancer Res. 71, 3121-3131.

Amente, S., Lania, L., and Majello, B. (2013). The histone LSD1 demethylase in stemness and cancer transcription programs. Biochim. Biophys. Acta BBA - Gene Regul. Mech. 1829, 981-986.

Anders, S., and Huber, W. (2010). Differential expression analysis for sequence count data. Genome Biol. 11, R106.

Arrowsmith, C.H., Bountra, C., Fish, P.V., Lee, K., and Schapira, M. (2012). Epigenetic protein families: a new frontier for drug discovery. Nat. Rev. Drug Discov. 11, 384-400.

Avvakumov, N., Nourani, A., and Côté, J. (2011). Histone Chaperones: Modulators of Chromatin Marks. Mol. Cell 41, 502-514.

Azuara, V., Perry, P., Sauer, S., Spivakov, M., Jørgensen, H.F., John, R.M., Gouti, M., Casanova, M., Warnes, G., Merkenschlager, M., et al. (2006). Chromatin signatures of pluripotent cell lines. Nat. Cell Biol. 8, 532-538.

Baillat, D., Gardini, A., Cesaroni, M., and Shiekhattar, R. (2012). Requirement for SNAPC1 in transcriptional responsiveness to diverse extracellular signals. Mol. Cell. Biol. 32, 46424650 .

Barker, N., Hurlstone, A., Musisi, H., Miles, A., Bienz, M., and Clevers, H. (2001). The chromatin remodelling factor Brg-1 interacts with $\beta$-catenin to promote target gene activation. EMBO J. 20, 4935-4943. 
Barretina, J., Caponigro, G., Stransky, N., Venkatesan, K., Margolin, A.A., Kim, S., Wilson, C.J., Lehár, J., Kryukov, G.V., Sonkin, D., et al. (2012). The Cancer Cell Line Encyclopedia enables predictive modelling of anticancer drug sensitivity. Nature 483, 603-607.

Bartel, D.P. (2004). MicroRNAs: genomics, biogenesis, mechanism, and function. Cell 116, 281-297.

Batlle, E., Sancho, E., Francí, C., Domínguez, D., Monfar, M., Baulida, J., and García De Herreros, A. (2000). The transcription factor snail is a repressor of E-cadherin gene expression in epithelial tumour cells. Nat. Cell Biol. 2, 84-89.

Baylin, S.B., Esteller, M., Rountree, M.R., Bachman, K.E., Schuebel, K., and Herman, J.G. (2001). Aberrant patterns of DNA methylation, chromatin formation and gene expression in cancer. Hum. Mol. Genet. 10, 687-692.

Bellan, C., De Falco, G., Lazzi, S., Micheli, P., Vicidomini, S., Schürfeld, K., Amato, T., Palumbo, A., Bagella, L., Sabattini, E., et al. (2004). CDK9/CYCLIN T1 expression during normal lymphoid differentiation and malignant transformation. J. Pathol. 203, 946-952.

Bernstein, B.E., Mikkelsen, T.S., Xie, X., Kamal, M., Huebert, D.J., Cuff, J., Fry, B., Meissner, A., Wernig, M., Plath, K., et al. (2006). A Bivalent Chromatin Structure Marks Key Developmental Genes in Embryonic Stem Cells. Cell 125, 315-326.

Birchmeier, W., and Behrens, J. (1994). Cadherin expression in carcinomas: role in the formation of cell junctions and the prevention of invasiveness. Biochim. Biophys. Acta 1198, $11-26$.

Bortvin, A., and Winston, F. (1996). Evidence that Spt6p controls chromatin structure by a direct interaction with histones. Science 272, 1473-1476.

Bos, P.D., Nguyen, D.X., and Massagué, J. (2010). Modeling metastasis in the mouse. Curr. Opin. Pharmacol. 10, 571-577.

Bunone, G., Briand, P.A., Miksicek, R.J., and Picard, D. (1996). Activation of the unliganded estrogen receptor by EGF involves the MAP kinase pathway and direct phosphorylation. EMBO J. 15, 2174-2183.

Buus, R., Faronato, M., Hammond, D.E., Urbé, S., and Clague, M.J. (2009). Deubiquitinase Activities Required for Hepatocyte Growth Factor-Induced Scattering of Epithelial Cells. Curr. Biol. 19, 1463-1466.

Byles, V., Zhu, L., Lovaas, J.D., Chmilewski, L.K., Wang, J., Faller, D.V., and Dai, Y. (2012). SIRT1 induces EMT by cooperating with EMT transcription factors and enhances prostate cancer cell migration and metastasis. Oncogene 31, 4619-4629.

Campos, E.I., and Reinberg, D. (2009). Histones: annotating chromatin. Annu. Rev. Genet. 43, 559-599.

Cao, R., Tsukada, Y., and Zhang, Y. (2005). Role of Bmi-1 and Ring1A in H2A Ubiquitylation and Hox Gene Silencing. Mol. Cell 20, 845-854.

Carroll, J.S., Meyer, C.A., Song, J., Li, W., Geistlinger, T.R., Eeckhoute, J., Brodsky, A.S., Keeton, E.K., Fertuck, K.C., Hall, G.F., et al. (2006). Genome-wide analysis of estrogen receptor binding sites. Nat. Genet. 38, 1289-1297. 
Cedar, H., and Bergman, Y. (2009). Linking DNA methylation and histone modification: patterns and paradigms. Nat. Rev. Genet. 10, 295-304.

Chaffer, C.L., Marjanovic, N.D., Lee, T., Bell, G., Kleer, C.G., Reinhardt, F., D'Alessio, A.C., Young, R.A., and Weinberg, R.A. (2013). Poised chromatin at the ZEB1 promoter enables breast cancer cell plasticity and enhances tumorigenicity. Cell 154,61-74.

Chen, R., Keating, M.J., Gandhi, V., and Plunkett, W. (2005). Transcription inhibition by flavopiridol: mechanism of chronic lymphocytic leukemia cell death. Blood 106, 2513-2519.

Chen, S., Li, J., Wang, D.-L., and Sun, F.-L. (2012a). Histone H2B lysine 120 monoubiquitination is required for embryonic stem cell differentiation. Cell Res. 22, 14021405.

Chen, S., Ma, J., Wu, F., Xiong, L., Ma, H., Xu, W., Lv, R., Li, X., Villen, J., Gygi, S.P., et al. (2012b). The histone H3 Lys 27 demethylase JMJD3 regulates gene expression by impacting transcriptional elongation. Genes Dev. 26, 1364-1375.

Chen, S., Ma, J., Wu, F., Xiong, L.-J., Ma, H., Xu, W., Lv, R., Li, X., Villen, J., Gygi, S.P., et al. (2012c). The histone H3 Lys 27 demethylase JMJD3 regulates gene expression by impacting transcriptional elongation. Genes Dev. 26, 1364-1375.

Chernikova, S.B., and Brown, J.M. (2012). R-loops and genomic instability in Bre1 (RNF20/40)-deficient cells. Cell Cycle 11, 2980-2984.

Chernikova, S.B., Razorenova, O.V., Higgins, J.P., Sishc, B.J., Nicolau, M., Dorth, J.A., Chernikova, D.A., Kwok, S., Brooks, J.D., Bailey, S.M., et al. (2012a). Deficiency in Mammalian Histone H2B Ubiquitin Ligase Bre1 (Rnf20/Rnf40) Leads to Replication Stress and Chromosomal Instability. Cancer Res. 72, 2111-2119.

Chernikova, S.B., Razorenova, O.V., Higgins, J.P., Sishc, B.J., Nicolau, M., Dorth, J.A., Chernikova, D.A., Kwok, S., Brooks, J.D., Bailey, S.M., et al. (2012b). Deficiency in Mammalian Histone H2B Ubiquitin Ligase Bre1 (Rnf20/Rnf40) Leads to Replication Stress and Chromosomal Instability. Cancer Res. 72, 2111-2119.

Chiang, P.-W., Wang, S., Smithivas, P., Song, W.-J., Ramamoorthy, S., Hillman, J., Puett, S., Van Keuren, M.L., Crombez, E., Kumar, A., et al. (1996). Identification and Analysis of the Human and Murine Putative Chromatin Structure Regulator SUPT6H andSupt6h. Genomics 34, 328-333.

Choe, M.K., Hong, C.-P., Park, J., Seo, S.H., and Roh, T.-Y. (2012). Functional elements demarcated by histone modifications in breast cancer cells. Biochem. Biophys. Res. Commun. 418, 475-482.

Collett, K., Eide, G.E., Arnes, J., Stefansson, I.M., Eide, J., Braaten, A., Aas, T., Otte, A.P., and Akslen, L.A. (2006). Expression of enhancer of zeste homologue 2 is significantly associated with increased tumor cell proliferation and is a marker of aggressive breast cancer. Clin. Cancer Res. Off. J. Am. Assoc. Cancer Res. 12, 1168-1174.

Couse, J.F. (1999). Estrogen Receptor Null Mice: What Have We Learned and Where Will They Lead Us? Endocr. Rev. 20, 358-417.

Couse, J.F., Curtis Hewitt, S., and Korach, K.S. (2000). Receptor null mice reveal contrasting roles for estrogen receptor alpha and beta in reproductive tissues. J. Steroid Biochem. Mol. Biol. 74, 287-296. 
Crawford, N.P.S., Alsarraj, J., Lukes, L., Walker, R.C., Officewala, J.S., Yang, H.H., Lee, M.P., Ozato, K., and Hunter, K.W. (2008). Bromodomain 4 activation predicts breast cancer survival. Proc. Natl. Acad. Sci. 105, 6380-6385.

Cromer, A., Carles, A., Millon, R., Ganguli, G., Chalmel, F., Lemaire, F., Young, J., Dembélé, D., Thibault, C., Muller, D., et al. (2004). Identification of genes associated with tumorigenesis and metastatic potential of hypopharyngeal cancer by microarray analysis. Oncogene 23, 2484-2498.

De la Cruz, X., Lois, S., Sánchez-Molina, S., and Martínez-Balbás, M.A. (2005). Do protein motifs read the histone code? BioEssays 27, 164-175.

Daniel, J.A., Torok, M.S., Sun, Z.-W., Schieltz, D., Allis, C.D., Yates, J.R., and Grant, P.A. (2004). Deubiquitination of Histone H2B by a Yeast Acetyltransferase Complex Regulates Transcription. J. Biol. Chem. 279, 1867-1871.

Danielian, P.S., White, R., Lees, J.A., and Parker, M.G. (1992). Identification of a conserved region required for hormone dependent transcriptional activation by steroid hormone receptors. EMBO J. 11, 1025-1033.

Das, C., Tyler, J.K., and Churchill, M.E.A. (2010). The histone shuffle: histone chaperones in an energetic dance. Trends Biochem. Sci. 35, 476-489.

Dauvois, S., White, R., and Parker, M.G. (1993). The antiestrogen ICI 182780 disrupts estrogen receptor nucleocytoplasmic shuttling. J. Cell Sci. 106, 1377-1388.

Delmore, J.E., Issa, G.C., Lemieux, M.E., Rahl, P.B., Shi, J., Jacobs, H.M., Kastritis, E., Gilpatrick, T., Paranal, R.M., Qi, J., et al. (2011). BET bromodomain inhibition as a therapeutic strategy to target c-Myc. Cell 146, 904-917.

Dengl, S., Mayer, A., Sun, M., and Cramer, P. (2009). Structure and in Vivo Requirement of the Yeast Spt6 SH2 Domain. J. Mol. Biol. 389, 211-225.

Deroo, B.J., and Korach, K.S. (2006). Estrogen receptors and human disease. J. Clin. Invest. 116, 561-570.

Devaiah, B.N., Lewis, B.A., Cherman, N., Hewitt, M.C., Albrecht, B.K., Robey, P.G., Ozato, K., Sims, R.J., and Singer, D.S. (2012). BRD4 is an atypical kinase that phosphorylates Serine2 of the RNA Polymerase II carboxy-terminal domain. Proc. Natl. Acad. Sci. 109, 6927-6932.

Dey, A., Ellenberg, J., Farina, A., Coleman, A.E., Maruyama, T., Sciortino, S., LippincottSchwartz, J., and Ozato, K. (2000). A Bromodomain Protein, MCAP, Associates with Mitotic Chromosomes and Affects G2-to-M Transition. Mol. Cell. Biol. 20, 6537-6549.

Dey, A., Chitsaz, F., Abbasi, A., Misteli, T., and Ozato, K. (2003). The double bromodomain protein Brd4 binds to acetylated chromatin during interphase and mitosis. Proc. Natl. Acad. Sci. 100, 8758-8763.

Dey, A., Nishiyama, A., Karpova, T., McNally, J., and Ozato, K. (2009). Brd4 Marks Select Genes on Mitotic Chromatin and Directs Postmitotic Transcription. Mol. Biol. Cell 20, 48994909. 
Diebold, M.-L., Loeliger, E., Koch, M., Winston, F., Cavarelli, J., and Romier, C. (2010). Noncanonical Tandem SH2 Enables Interaction of Elongation Factor Spt6 with RNA Polymerase II. J. Biol. Chem. 285, 38389-38398.

Dong, C., Wu, Y., Yao, J., Wang, Y., Yu, Y., Rychahou, P.G., Evers, B.M., and Zhou, B.P. (2012). G9a interacts with Snail and is critical for Snail-mediated E-cadherin repression in human breast cancer. J. Clin. Invest. 122, 1469-1486.

Dong, C., Wu, Y., Wang, Y., Wang, C., Kang, T., Rychahou, P.G., Chi, Y.-I., Evers, B.M., and Zhou, B.P. (2013). Interaction with Suv39H1 is critical for Snail-mediated E-cadherin repression in breast cancer. Oncogene 32, 1351-1362.

Dumont, N., Wilson, M.B., Crawford, Y.G., Reynolds, P.A., Sigaroudinia, M., and Tlsty, T.D. (2008). Sustained induction of epithelial to mesenchymal transition activates DNA methylation of genes silenced in basal-like breast cancers. Proc. Natl. Acad. Sci. U. S. A. $105,14867-14872$.

Dupont, C., Armant, D.R., and Brenner, C.A. (2009). Epigenetics: definition, mechanisms and clinical perspective. Semin. Reprod. Med. 27, 351-357.

Egloff, S., and Murphy, S. (2008a). Cracking the RNA polymerase II CTD code. Trends Genet. TIG 24, 280-288.

Egloff, S., and Murphy, S. (2008b). Cracking the RNA polymerase II CTD code. Trends Genet. 24, 280-288.

Egloff, S., Herreweghe, E.V., and Kiss, T. (2006). Regulation of Polymerase II Transcription by 7SK snRNA: Two Distinct RNA Elements Direct P-TEFb and HEXIM1 Binding. Mol. Cell. Biol. 26, 630-642.

Abd El-Rehim, D.M., Pinder, S.E., Paish, C.E., Bell, J., Blamey, R., Robertson, J.F., Nicholson, R.I., and Ellis, I.O. (2004). Expression of luminal and basal cytokeratins in human breast carcinoma. J. Pathol. 203, 661-671.

Emmen, J.M.A., and Korach, K.S. (2009). Estrogen receptor knockout mice: phenotypes in the female reproductive tract.

Emre, N.C.T., Ingvarsdottir, K., Wyce, A., Wood, A., Krogan, N.J., Henry, K.W., Li, K., Marmorstein, R., Greenblatt, J.F., Shilatifard, A., et al. (2005). Maintenance of Low Histone Ubiquitylation by Ubp10 Correlates with Telomere-Proximal Sir2 Association and Gene Silencing. Mol. Cell 17, 585-594.

Endoh, M., Zhu, W., Hasegawa, J., Watanabe, H., Kim, D.-K., Aida, M., Inukai, N., Narita, T., Yamada, T., Furuya, A., et al. (2004). Human Spt6 stimulates transcription elongation by RNA polymerase II in vitro. Mol. Cell. Biol. 24, 3324-3336.

Falco, G.D., and Giordano, A. CDK9: From Basal Transcription to Cancer and AIDS. Cancer Biol. Ther. 1, 341-346.

Farley, F.W., Soriano, P., Steffen, L.S., and Dymecki, S.M. (2000). Widespread recombinase expression using FLPeR (flipper) mice. Genes. N. Y. N 2000 28, 106-110.

Ferrari-Amorotti, G., Fragliasso, V., Esteki, R., Prudente, Z., Soliera, A.R., Cattelani, S., Manzotti, G., Grisendi, G., Dominici, M., Pieraccioli, M., et al. (2013). Inhibiting Interactions 
of Lysine Demethylase LSD1 with Snail/Slug Blocks Cancer Cell Invasion. Cancer Res. 73, 235-245.

Fierz, B., Chatterjee, C., McGinty, R.K., Bar-Dagan, M., Raleigh, D.P., and Muir, T.W. (2011a). Histone H2B ubiquitylation disrupts local and higher-order chromatin compaction. Nat. Chem. Biol. 7, 113-119.

Fierz, B., Chatterjee, C., McGinty, R.K., Bar-Dagan, M., Raleigh, D.P., and Muir, T.W. (2011b). Histone H2B ubiquitylation disrupts local and higher-order chromatin compaction. Nat. Chem. Biol. 7, 113-119.

Filippakopoulos, P., Qi, J., Picaud, S., Shen, Y., Smith, W.B., Fedorov, O., Morse, E.M., Keates, T., Hickman, T.T., Felletar, I., et al. (2010). Selective inhibition of BET bromodomains. Nature 468, 1067-1073.

Finak, G., Bertos, N., Pepin, F., Sadekova, S., Souleimanova, M., Zhao, H., Chen, H., Omeroglu, G., Meterissian, S., Omeroglu, A., et al. (2008). Stromal gene expression predicts clinical outcome in breast cancer. Nat. Med. 14, 518-527.

Fleming, A.B., Kao, C.-F., Hillyer, C., Pikaart, M., and Osley, M.A. (2008). H2B Ubiquitylation Plays a Role in Nucleosome Dynamics during Transcription Elongation. Mol. Cell 31, 57-66.

Florence, B., and Faller, D.V. (2001). You bet-cha: a novel family of transcriptional regulators. Front. Biosci. J. Virtual Libr. 6, D1008-1018.

Foskett, S.M., Ghose, R., Tang, D.N., Lewis, D.E., and Rice, A.P. (2001). Antiapoptotic Function of Cdk9 (TAK/P-TEFb) in U937 Promonocytic Cells. J. Virol. 75, 1220-1228.

Francis, N.J., Kingston, R.E., and Woodcock, C.L. (2004). Chromatin Compaction by a Polycomb Group Protein Complex. Science 306, 1574-1577.

French, C.A., Miyoshi, I., Aster, J.C., Kubonishi, I., Kroll, T.G., Dal Cin, P., Vargas, S.O., Perez-Atayde, A.R., and Fletcher, J.A. (2001). BRD4 Bromodomain Gene Rearrangement in Aggressive Carcinoma with Translocation t(15;19). Am. J. Pathol. 159, 1987-1992.

French, C.A., Miyoshi, I., Kubonishi, I., Grier, H.E., Perez-Atayde, A.R., and Fletcher, J.A. (2003). BRD4-NUT Fusion Oncogene A Novel Mechanism in Aggressive Carcinoma. Cancer Res. 63, 304-307.

French, C.A., Kutok, J.L., Faquin, W.C., Toretsky, J.A., Antonescu, C.R., Griffin, C.A., Nose, V., Vargas, S.O., Moschovi, M., Tzortzatou-Stathopoulou, F., et al. (2004). Midline Carcinoma of Children and Young Adults With NUT Rearrangement. J. Clin. Oncol. 22, 4135-4139.

French, C.A., Ramirez, C.L., Kolmakova, J., Hickman, T.T., Cameron, M.J., Thyne, M.E., Kutok, J.L., Toretsky, J.A., Tadavarthy, A.K., Kees, U.R., et al. (2007). BRD-NUT oncoproteins: a family of closely related nuclear proteins that block epithelial differentiation and maintain the growth of carcinoma cells. Oncogene 27, 2237-2242.

Fu, T.-J., Peng, J., Lee, G., Price, D.H., and Flores, O. (1999). Cyclin K Functions as a CDK9 Regulatory Subunit and Participates in RNA Polymerase II Transcription. J. Biol. Chem. 274, 34527-34530. 
Fuchs, G., Shema, E., Vesterman, R., Kotler, E., Wolchinsky, Z., Wilder, S., Golomb, L., Pribluda, A., Zhang, F., Haj-Yahya, M., et al. (2012). RNF20 and USP44 regulate stem cell differentiation by modulating H2B monoubiquitylation. Mol. Cell 46, 662-673.

Fujinaga, K., Irwin, D., Huang, Y., Taube, R., Kurosu, T., and Peterlin, B.M. (2004). Dynamics of Human Immunodeficiency Virus Transcription: P-TEFb Phosphorylates RD and Dissociates Negative Effectors from the Transactivation Response Element. Mol. Cell. Biol. 24, 787-795.

Fujita, N., Jaye, D.L., Kajita, M., Geigerman, C., Moreno, C.S., and Wade, P.A. (2003). MTA3, a Mi-2/NuRD Complex Subunit, Regulates an Invasive Growth Pathway in Breast Cancer. Cell 113, 207-219.

Fullwood, M.J., Liu, M.H., Pan, Y.F., Liu, J., Xu, H., Mohamed, Y.B., Orlov, Y.L., Velkov, S., Ho, A., Mei, P.H., et al. (2009). An oestrogen-receptor-alpha-bound human chromatin interactome. Nature 462, 58-64.

Gardner, R.G., Nelson, Z.W., and Gottschling, D.E. (2005). Ubp10/Dot4p Regulates the Persistence of Ubiquitinated Histone H2B: Distinct Roles in Telomeric Silencing and General Chromatin. Mol. Cell. Biol. 25, 6123-6139.

Glickman, M.H., and Ciechanover, A. (2002). The Ubiquitin-Proteasome Proteolytic Pathway: Destruction for the Sake of Construction. Physiol. Rev. 82, 373-428.

Glinsky, G.V., Berezovska, O., and Glinskii, A.B. (2005). Microarray analysis identifies a death-from-cancer signature predicting therapy failure in patients with multiple types of cancer. J. Clin. Invest. 115, 1503-1521.

Glover-Cutter, K., Larochelle, S., Erickson, B., Zhang, C., Shokat, K., Fisher, R.P., and Bentley, D.L. (2009). TFIIH-Associated Cdk7 Kinase Functions in Phosphorylation of CTerminal Domain Ser7 Residues, Promoter-Proximal Pausing, and Termination by RNA Polymerase II. Mol. Cell. Biol. 29, 5455-5464.

Gotzmann, J., Fischer, A.N.M., Zojer, M., Mikula, M., Proell, V., Huber, H., Jechlinger, M., Waerner, T., Weith, A., Beug, H., et al. (2006). A crucial function of PDGF in TGF-betamediated cancer progression of hepatocytes. Oncogene 25, 3170-3185.

Grant, P.A., Duggan, L., Côté, J., Roberts, S.M., Brownell, J.E., Candau, R., Ohba, R., Owen-Hughes, T., Allis, C.D., Winston, F., et al. (1997). Yeast Gcn5 functions in two multisubunit complexes to acetylate nucleosomal histones: characterization of an Ada complex and the SAGA (Spt/Ada) complex. Genes Dev. 11, 1640-1650.

Green, S., Walter, P., Kumar, V., Krust, A., Bornert, J.-M., Argos, P., and Chambon, P. (1986). Human oestrogen receptor cDNA: sequence, expression and homology to v-erb-A. Nature 320, 134-139.

Grimshaw, M.J., Cooper, L., Papazisis, K., Coleman, J.A., Bohnenkamp, H.R., ChiaperoStanke, L., Taylor-Papadimitriou, J., and Burchell, J.M. (2008). Mammosphere culture of metastatic breast cancer cells enriches for tumorigenic breast cancer cells. Breast Cancer Res. 10, R52.

Grunstein, M. (1997). Histone acetylation in chromatin structure and transcription. Nature 389, 349-352. 
Hahn, M.A., Dickson, K.-A., Jackson, S., Clarkson, A., Gill, A.J., and Marsh, D.J. (2012). The tumor suppressor CDC73 interacts with the ring finger proteins RNF20 and RNF40 and is required for the maintenance of histone 2B monoubiquitination. Hum. Mol. Genet. 21, 559568.

Al-Hajj, M., Wicha, M.S., Benito-Hernandez, A., Morrison, S.J., and Clarke, M.F. (2003a). Prospective identification of tumorigenic breast cancer cells. Proc. Natl. Acad. Sci. 100, 3983-3988.

Al-Hajj, M., Wicha, M.S., Benito-Hernandez, A., Morrison, S.J., and Clarke, M.F. (2003b). Prospective identification of tumorigenic breast cancer cells. Proc. Natl. Acad. Sci. 100, 3983-3988.

Hartzog, G.A., Wada, T., Handa, H., and Winston, F. (1998). Evidence that Spt4, Spt5, and Spt6 control transcription elongation by RNA polymerase II inSaccharomyces cerevisiae. Genes Dev. 12, 357-369.

Hawkins, R.D., Hon, G.C., Lee, L.K., Ngo, Q., Lister, R., Pelizzola, M., Edsall, L.E., Kuan, S., Luu, Y., Klugman, S., et al. (2010). Distinct epigenomic landscapes of pluripotent and lineage-committed human cells. Cell Stem Cell 6, 479-491.

Haynes, S.R., Dollard, C., Winston, F., Beck, S., Trowsdale, J., and Dawid, I.B. (1992). The bromodomain: a conserved sequence found in human, Drosophila and yeast proteins. Nucleic Acids Res. 20, 2603-2603.

Henry, K.W., Wyce, A., Lo, W.-S., Duggan, L.J., Emre, N.C.T., Kao, C.-F., Pillus, L., Shilatifard, A., Osley, M.A., and Berger, S.L. (2003). Transcriptional activation via sequential histone H2B ubiquitylation and deubiquitylation, mediated by SAGA-associated Ubp8. Genes Dev. 17, 2648-2663.

Herranz, N., Pasini, D., Díaz, V.M., Francí, C., Gutierrez, A., Dave, N., Escrivà, M., Hernandez-Muñoz, I., Di Croce, L., Helin, K., et al. (2008). Polycomb complex 2 is required for E-cadherin repression by the Snail1 transcription factor. Mol. Cell. Biol. 28, 4772-4781.

Hershko, A., and Ciechanover, A. (1998). The Ubiquitin System. Annu. Rev. Biochem. 67, 425-479.

Hewitt, S.C., Couse, J.F., and Korach, K.S. (2000). Estrogen receptor transcription and transactivation: Estrogen receptor knockout mice - what their phenotypes reveal about mechanisms of estrogen action. Breast Cancer Res. 2, 345.

Hochstrasser, M. (1996). Ubiquitin-Dependent Protein Degradation. Annu. Rev. Genet. 30, 405-439.

Houzelstein, D., Bullock, S.L., Lynch, D.E., Grigorieva, E.F., Wilson, V.A., and Beddington, R.S.P. (2002). Growth and Early Postimplantation Defects in Mice Deficient for the Bromodomain-Containing Protein Brd4t. Mol. Cell. Biol. 22, 3794-3802.

Hsu, C.-H., Peng, K.-L., Kang, M.-L., Chen, Y.-R., Yang, Y.-C., Tsai, C.-H., Chu, C.-S., Jeng, Y.-M., Chen, Y.-T., Lin, F.-M., et al. (2012). TET1 suppresses cancer invasion by activating the tissue inhibitors of metalloproteinases. Cell Rep. 2, 568-579.

Huang, B., Yang, X.-D., Zhou, M.-M., Ozato, K., and Chen, L.-F. (2009). Brd4 Coactivates Transcriptional Activation of NF-?B via Specific Binding to Acetylated RelA. Mol. Cell. Biol. 29, 1375-1387. 
Huang, H., Jiang, X., Li, Z., Li, Y., Song, C.-X., He, C., Sun, M., Chen, P., Gurbuxani, S., Wang, J., et al. (2013). TET1 plays an essential oncogenic role in MLL-rearranged leukemia. Proc. Natl. Acad. Sci. U. S. A. 110, 11994-11999.

Hwang, W.W., Venkatasubrahmanyam, S., lanculescu, A.G., Tong, A., Boone, C., and Madhani, H.D. (2003). A Conserved RING Finger Protein Required for Histone H2B Monoubiquitination and Cell Size Control. Mol. Cell 11, 261-266.

Imhof, A., Yang, X.-J., Ogryzko, V.V., Nakatani, Y., Wolffe, A.P., and Ge, H. (1997). Acetylation of general transcription factors by histone acetyltransferases. Curr. Biol. 7, 689692.

Jaeger, J., Koczan, D., Thiesen, H.-J., Ibrahim, S.M., Gross, G., Spang, R., and Kunz, M. (2007). Gene expression signatures for tumor progression, tumor subtype, and tumor thickness in laser-microdissected melanoma tissues. Clin. Cancer Res. Off. J. Am. Assoc. Cancer Res. 13, 806-815.

Jafarnejad, S.M., and Li, G. (2012). Regulation of p53 by ING family members in suppression of tumor initiation and progression. Cancer Metastasis Rev. 31, 55-73.

James, L.I., and Frye, S.V. (2013). Targeting Chromatin Readers. Clin. Pharmacol. Ther. 93, 312-314.

Jang, M.K., Mochizuki, K., Zhou, M., Jeong, H.-S., Brady, J.N., and Ozato, K. (2005a). The Bromodomain Protein Brd4 Is a Positive Regulatory Component of P-TEFb and Stimulates RNA Polymerase II-Dependent Transcription. Mol. Cell 19, 523-534.

Jang, M.K., Mochizuki, K., Zhou, M., Jeong, H.-S., Brady, J.N., and Ozato, K. (2005b). The Bromodomain Protein Brd4 Is a Positive Regulatory Component of P-TEFb and Stimulates RNA Polymerase II-Dependent Transcription. Mol. Cell 19, 523-534.

Jeanmougin, F., Wurtz, J.-M., Le Douarin, B., Chambon, P., and Losson, R. (1997). The bromodomain revisited. Trends Biochem. Sci. 22, 151-153.

Jechlinger, M., Grunert, S., Tamir, I.H., Janda, E., Lüdemann, S., Waerner, T., Seither, P., Weith, A., Beug, H., and Kraut, N. (2003). Expression profiling of epithelial plasticity in tumor progression. Oncogene 22, 7155-7169.

Jensen, E.V., and DeSombre, E.R. (1973). Estrogen-Receptor Interaction Estrogenic hormones effect transformation of specific receptor proteins to a biochemically functional form. Science 182, 126-134.

Jenuwein, T., and Allis, C.D. (2001). Translating the histone code. Science 293, 1074-1080.

Johnsen, S.A. (2012a). The enigmatic role of H2Bub1 in cancer. FEBS Lett. 586, 15921601.

Johnsen, S.A. (2012b). The enigmatic role of H2Bub1 in cancer. FEBS Lett. 586, $1592-$ 1601.

Johnsen, S.A. (2012c). CDK9 and H2B monoubiquitination: a well-choreographed dance. PLoS Genet. 8, e1002860. 
Johnsen, S.A., Kangaspeska, S., Reid, G., and Gannon, F. (2006). Interfering with the dynamics of estrogen receptor-regulated transcription. Ernst Schering Found. Symp. Proc. $1-12$.

Jones, P.A., and Baylin, S.B. (2007). The epigenomics of cancer. Cell 128, 683-692.

K, P., and U, W. (2003). Micrometastasis in breast cancer and other solid tumors. J. Biol. Regul. Homeost. Agents 18, 120-125.

Kamalakaran, S., Varadan, V., Giercksky Russnes, H.E., Levy, D., Kendall, J., Janevski, A., Riggs, M., Banerjee, N., Synnestvedt, M., Schlichting, E., et al. (2011). DNA methylation patterns in luminal breast cancers differ from non-luminal subtypes and can identify relapse risk independent of other clinical variables. Mol. Oncol. 5, 77-92.

Kangaspeska, S., Stride, B., Métivier, R., Polycarpou-Schwarz, M., Ibberson, D., Carmouche, R.P., Benes, V., Gannon, F., and Reid, G. (2008). Transient cyclical methylation of promoter DNA. Nature 452, 112-115.

Kanno, T., Kanno, Y., Siegel, R.M., Jang, M.K., Lenardo, M.J., and Ozato, K. (2004).

Selective Recognition of Acetylated Histones by Bromodomain Proteins Visualized in Living Cells. Mol. Cell 13, 33-43.

Kaplan, C.D., Laprade, L., and Winston, F. (2003). Transcription elongation factors repress transcription initiation from cryptic sites. Science 301, 1096-1099.

Kari, V., Shchebet, A., Neumann, H., and Johnsen, S.A. (2011a). The H2B ubiquitin ligase RNF40 cooperates with SUPT16H to induce dynamic changes in chromatin structure during DNA double-strand break repair. Cell Cycle Georget. Tex 10, 3495-3504.

Kari, V., Shchebet, A., Neumann, H., and Johnsen, S.A. (2011b). The H2B ubiquitin ligase RNF40 cooperates with SUPT16 $\mathrm{H}$ to induce dynamic changes in chromatin structure during DNA double-strand break repair. Cell Cycle Georget. Tex 10, 3495-3504.

Karpiuk, O., Najafova, Z., Kramer, F., Hennion, M., Galonska, C., König, A., Snaidero, N., Vogel, T., Shchebet, A., Begus-Nahrmann, Y., et al. (2012a). The histone H2B monoubiquitination regulatory pathway is required for differentiation of multipotent stem cells. Mol. Cell 46, 705-713.

Karpiuk, O., Najafova, Z., Kramer, F., Hennion, M., Galonska, C., König, A., Snaidero, N., Vogel, T., Shchebet, A., Begus-Nahrmann, Y., et al. (2012b). The Histone H2B Monoubiquitination Regulatory Pathway Is Required for Differentiation of Multipotent Stem Cells. Mol. Cell 46, 705-713.

Kaufman, P.D. (1996). Nucleosome assembly: the CAF and the HAT. Curr. Opin. Cell Biol. 8, 369-373.

Ketchart, W., Ogba, N., Kresak, A., Albert, J.M., Pink, J.J., and Montano, M.M. (2011). HEXIM1 is a critical determinant of the response to tamoxifen. Oncogene 30, 3563-3569.

Khan, S.A., Rogers, M.A.M., Khurana, K.K., Meguid, M.M., and Numann, P.J. (1998). Estrogen Receptor Expression in Benign Breast Epithelium and Breast Cancer Risk. J. Natl. Cancer Inst. 90, 37-42.

Kilpinen, S., Autio, R., Ojala, K., Iljin, K., Bucher, E., Sara, H., Pisto, T., Saarela, M., Skotheim, R.I., Björkman, M., et al. (2008). Systematic bioinformatic analysis of expression 
levels of 17,330 human genes across 9,783 samples from 175 types of healthy and pathological tissues. Genome Biol. 9, R139.

Kim, J., Hake, S.B., and Roeder, R.G. (2005). The Human Homolog of Yeast BRE1 Functions as a Transcriptional Coactivator through Direct Activator Interactions. Mol. Cell 20, 759-770.

Kim, J., Daniel, J., Espejo, A., Lake, A., Krishna, M., Xia, L., Zhang, Y., and Bedford, M.T. (2006). Tudor, MBT and chromo domains gauge the degree of lysine methylation. EMBO Rep. 7, 397-403.

Kim, J., Guermah, M., McGinty, R.K., Lee, J.-S., Tang, Z., Milne, T.A., Shilatifard, A., Muir, T.W., and Roeder, R.G. (2009a). RAD6-Mediated Transcription-Coupled H2B Ubiquitylation Directly Stimulates H3K4 Methylation in Human Cells. Cell 137, 459-471.

Kim, J., Guermah, M., McGinty, R.K., Lee, J.-S., Tang, Z., Milne, T.A., Shilatifard, A., Muir, T.W., and Roeder, R.G. (2009b). RAD6-Mediated transcription-coupled H2B ubiquitylation directly stimulates H3K4 methylation in human cells. Cell 137, 459-471.

Kim, T.-K., Hemberg, M., Gray, J.M., Costa, A.M., Bear, D.M., Wu, J., Harmin, D.A., Laptewicz, M., Barbara-Haley, K., Kuersten, S., et al. (2010). Widespread transcription at neuronal activity-regulated enhancers. Nature 465, 182-187.

Kininis, M., Isaacs, G.D., Core, L.J., Hah, N., and Kraus, W.L. (2009). Postrecruitment regulation of RNA polymerase II directs rapid signaling responses at the promoters of estrogen target genes. Mol. Cell. Biol. 29, 1123-1133.

Köhler, A., Pascual-García, P., Llopis, A., Zapater, M., Posas, F., Hurt, E., and RodríguezNavarro, S. (2006). The mRNA Export Factor Sus1 Is Involved in Spt/Ada/Gcn5 Acetyltransferase-mediated H2B Deubiquitinylation through Its Interaction with Ubp8 and Sgf11. Mol. Biol. Cell 17, 4228-4236.

Kokura, K., Sun, L., Bedford, M.T., and Fang, J. (2010). Methyl-H3K9-binding protein MPP8 mediates E-cadherin gene silencing and promotes tumour cell motility and invasion. EMBO J. 29, 3673-3687.

Koman, I.E., Commane, M., Paszkiewicz, G., Hoonjan, B., Pal, S., Safina, A., Toshkov, I., Purmal, A.A., Wang, D., Liu, S., et al. (2012). Targeting FACT Complex Suppresses Mammary Tumorigenesis in Her2/neu Transgenic Mice. Cancer Prev. Res. (Phila. Pa.) 5, 1025-1035.

Korpal, M., Lee, E.S., Hu, G., and Kang, Y. (2008). The miR-200 Family Inhibits EpithelialMesenchymal Transition and Cancer Cell Migration by Direct Targeting of E-cadherin Transcriptional Repressors ZEB1 and ZEB2. J. Biol. Chem. 283, 14910-14914.

Kouzarides, T. (2007). Chromatin modifications and their function. Cell 128, 693-705.

Krubasik, D., Iyer, N.G., English, W.R., Ahmed, A.A., Vias, M., Roskelley, C., Brenton, J.D., Caldas, C., and Murphy, G. (2006). Absence of p300 induces cellular phenotypic changes characteristic of epithelial to mesenchyme transition. Br. J. Cancer 94, 1326-1332.

Kuiper, G.G., Enmark, E., Pelto-Huikko, M., Nilsson, S., and Gustafsson, J.A. (1996). Cloning of a novel receptor expressed in rat prostate and ovary. Proc. Natl. Acad. Sci. 93, 5925-5930. 
Lachner, M., and Jenuwein, T. (2002). The many faces of histone lysine methylation. Curr. Opin. Cell Biol. 14, 286-298.

Laemmli, U.K. (1970). Cleavage of structural proteins during the assembly of the head of bacteriophage T4. Nature 227, 680-685.

Lang, G., Bonnet, J., Umlauf, D., Karmodiya, K., Koffler, J., Stierle, M., Devys, D., and Tora, L. (2011). The Tightly Controlled Deubiquitination Activity of the Human SAGA Complex Differentially Modifies Distinct Gene Regulatory Elements. Mol. Cell. Biol. 31, 3734-3744.

Lee, D.K., Duan, H.O., and Chang, C. (2001). Androgen Receptor Interacts with the Positive Elongation Factor P-TEFb and Enhances the Efficiency of Transcriptional Elongation. J. Biol. Chem. 276, 9978-9984.

Lee, J.-S., Shukla, A., Schneider, J., Swanson, S.K., Washburn, M.P., Florens, L., Bhaumik, S.R., and Shilatifard, A. (2007). Histone Crosstalk between H2B Monoubiquitination and H3 Methylation Mediated by COMPASS. Cell 131, 1084-1096.

Lees, J.A., Fawell, S.E., and Parker, M.G. (1989). Identification of two transactlvation domains in the mouse oestrogen receptor. Nucleic Acids Res. 17, 5477-5488.

Lei, W., Zhang, K., Pan, X., Hu, Y., Wang, D., Yuan, X., Shu, G., and Song, J. (2010). Histone deacetylase 1 is required for transforming growth factor- $\beta 1$-induced epithelialmesenchymal transition. Int. J. Biochem. Cell Biol. 42, 1489-1497.

Levine, S.S., Weiss, A., Erdjument-Bromage, H., Shao, Z., Tempst, P., and Kingston, R.E. (2002). The Core of the Polycomb Repressive Complex Is Compositionally and Functionally Conserved in Flies and Humans. Mol. Cell. Biol. 22, 6070-6078.

Li, B., Carey, M., and Workman, J.L. (2007a). The Role of Chromatin during Transcription. Cell 128, 707-719.

Li, B., Carey, M., and Workman, J.L. (2007b). The Role of Chromatin during Transcription. Cell 128, 707-719.

Li, Q., Price, J.P., Byers, S.A., Cheng, D., Peng, J., and Price, D.H. (2005). Analysis of the Large Inactive P-TEFb Complex Indicates That It Contains One 7SK Molecule, a Dimer of HEXIM1 or HEXIM2, and Two P-TEFb Molecules Containing Cdk9 Phosphorylated at Threonine 186. J. Biol. Chem. 280, 28819-28826.

Li, W., Notani, D., Ma, Q., Tanasa, B., Nunez, E., Chen, A.Y., Merkurjev, D., Zhang, J., Ohgi, K., Song, X., et al. (2013). Functional roles of enhancer RNAs for oestrogen-dependent transcriptional activation. Nature 498, 516-520.

Lim, E., Wu, D., Pal, B., Bouras, T., Asselin-Labat, M.-L., Vaillant, F., Yagita, H., Lindeman, G.J., Smyth, G.K., and Visvader, J.E. (2010). Transcriptome analyses of mouse and human mammary cell subpopulations reveal multiple conserved genes and pathways. Breast Cancer Res. BCR 12, R21.

Lin, T., Ponn, A., Hu, X., Law, B.K., and Lu, J. (2010a). Requirement of the histone demethylase LSD1 in Snai1-mediated transcriptional repression during epithelialmesenchymal transition. Oncogene 29, 4896-4904. 
Lin, Y., Wu, Y., Li, J., Dong, C., Ye, X., Chi, Y.-I., Evers, B.M., and Zhou, B.P. (2010b). The SNAG domain of Snail1 functions as a molecular hook for recruiting lysine-specific demethylase 1. EMBO J. 29, 1803-1816.

Liu, P., Ramachandran, S., Ali Seyed, M., Scharer, C.D., Laycock, N., Dalton, W.B., Williams, H., Karanam, S., Datta, M.W., Jaye, D.L., et al. (2006). Sex-determining region Y box 4 is a transforming oncogene in human prostate cancer cells. Cancer Res. 66, 40114019.

Liu, T., Ortiz, J.A., Taing, L., Meyer, C.A., Lee, B., Zhang, Y., Shin, H., Wong, S.S., Ma, J., Lei, Y., et al. (2011a). Cistrome: an integrative platform for transcriptional regulation studies. Genome Biol. 12, R83.

Liu, Y.-L., Yang, Y.-M., Xu, H., and Dong, X.-S. (2011b). Aberrant expression of USP22 is associated with liver metastasis and poor prognosis of colorectal cancer. J. Surg. Oncol. 103, 283-289.

Liu, Y.-L., Jiang, S.-X., Yang, Y.-M., Xu, H., Liu, J.-L., and Wang, X.-S. (2012). USP22 Acts as an Oncogene by the Activation of BMI-1-Mediated INK4a/ARF Pathway and Akt Pathway. Cell Biochem. Biophys. 62, 229-235.

Lombaerts, M., van Wezel, T., Philippo, K., Dierssen, J.W.F., Zimmerman, R.M.E., Oosting, J., van Eijk, R., Eilers, P.H., van de Water, B., Cornelisse, C.J., et al. (2006). E-cadherin transcriptional downregulation by promoter methylation but not mutation is related to epithelial-to-mesenchymal transition in breast cancer cell lines. Br. J. Cancer 94, 661-671.

Long, J., Zuo, D., and Park, M. (2005). Pc2-mediated sumoylation of Smad-interacting protein 1 attenuates transcriptional repression of E-cadherin. J. Biol. Chem. 280, 3547735489.

Loyola, A., and Almouzni, G. (2004). Histone chaperones, a supporting role in the limelight. Biochim. Biophys. Acta BBA - Gene Struct. Expr. 1677, 3-11.

Lydon, J.P., and O'Malley, B.W. (2011). Minireview: Steroid Receptor Coactivator-3: A Multifarious Coregulator in Mammary Gland Metastasis. Endocrinology 152, 19-25.

Mani, S.A., Guo, W., Liao, M.-J., Eaton, E.N., Ayyanan, A., Zhou, A.Y., Brooks, M., Reinhard, F., Zhang, C.C., Shipitsin, M., et al. (2008). The Epithelial-Mesenchymal Transition Generates Cells with Properties of Stem Cells. Cell 133, 704-715.

Maruyama, R., Choudhury, S., Kowalczyk, A., Bessarabova, M., Beresford-Smith, B., Conway, T., Kaspi, A., Wu, Z., Nikolskaya, T., Merino, V.F., et al. (2011). Epigenetic regulation of cell type-specific expression patterns in the human mammary epithelium. PLoS Genet. 7, e1001369.

Maruyama, T., Farina, A., Dey, A., Cheong, J., Bermudez, V.P., Tamura, T., Sciortino, S., Shuman, J., Hurwitz, J., and Ozato, K. (2002). A Mammalian Bromodomain Protein, Brd4, Interacts with Replication Factor C and Inhibits Progression to S Phase. Mol. Cell. Biol. 22, 6509-6520.

McCabe, M.T., Brandes, J.C., and Vertino, P.M. (2009). Cancer DNA methylation: molecular mechanisms and clinical implications. Clin. Cancer Res. Off. J. Am. Assoc. Cancer Res. 15, 3927-3937. 
Métivier, R., Penot, G., Hübner, M.R., Reid, G., Brand, H., Kos, M., and Gannon, F. (2003). Estrogen receptor-alpha directs ordered, cyclical, and combinatorial recruitment of cofactors on a natural target promoter. Cell 115, 751-763.

Métivier, R., Reid, G., and Gannon, F. (2006). Transcription in four dimensions: nuclear receptor-directed initiation of gene expression. EMBO Rep. 7, 161-167.

Métivier, R., Gallais, R., Tiffoche, C., Le Péron, C., Jurkowska, R.Z., Carmouche, R.P., Ibberson, D., Barath, P., Demay, F., Reid, G., et al. (2008). Cyclical DNA methylation of a transcriptionally active promoter. Nature $452,45-50$.

Michels, A.A., Fraldi, A., Li, Q., Adamson, T.E., Bonnet, F., Nguyen, V.T., Sedore, S.C., Price, J.P., Price, D.H., Lania, L., et al. (2004). Binding of the 7SK snRNA turns the HEXIM1 protein into a P-TEFb (CDK9/cyclin T) inhibitor. EMBO J. 23, 2608-2619.

Miller, T., Krogan, N.J., Dover, J., Erdjument-Bromage, H., Tempst, P., Johnston, M., Greenblatt, J.F., and Shilatifard, A. (2001). COMPASS: A complex of proteins associated with a trithorax-related SET domain protein. Proc. Natl. Acad. Sci. 98, 12902-12907.

Minsky, N., Shema, E., Field, Y., Schuster, M., Segal, E., and Oren, M. (2008a).

Monoubiquitinated $\mathrm{H} 2 \mathrm{~B}$ is associated with the transcribed region of highly expressed genes in human cells. Nat. Cell Biol. 10, 483-488.

Minsky, N., Shema, E., Field, Y., Schuster, M., Segal, E., and Oren, M. (2008b).

Monoubiquitinated $\mathrm{H} 2 \mathrm{~B}$ is associated with the transcribed region of highly expressed genes in human cells. Nat. Cell Biol. 10, 483-488.

Mortazavi, A., Williams, B.A., McCue, K., Schaeffer, L., and Wold, B. (2008). Mapping and quantifying mammalian transcriptomes by RNA-Seq. Nat. Methods 5, 621-628.

Moyal, L., Lerenthal, Y., Gana-Weisz, M., Mass, G., So, S., Wang, S.-Y., Eppink, B., Chung, Y.M., Shalev, G., Shema, E., et al. (2011). Requirement of ATM-Dependent Monoubiquitylation of Histone H2B for Timely Repair of DNA Double-Strand Breaks. Mol. Cell 41, 529-542.

Mu, J.-J., Wang, Y., Luo, H., Leng, M., Zhang, J., Yang, T., Besusso, D., Jung, S.Y., and Qin, J. (2007). A Proteomic Analysis of Ataxia Telangiectasia-mutated (ATM)/ATM-Rad3related (ATR) Substrates Identifies the Ubiquitin-Proteasome System as a Regulator for DNA Damage Checkpoints. J. Biol. Chem. 282, 17330-17334.

Nakamura, K., Kato, A., Kobayashi, J., Yanagihara, H., Sakamoto, S., Oliveira, D.V.N.P., Shimada, M., Tauchi, H., Suzuki, H., Tashiro, S., et al. (2011). Regulation of Homologous Recombination by RNF20-Dependent H2B Ubiquitination. Mol. Cell 41, 515-528.

Nicassio, F., Corrado, N., Vissers, J.H.A., Areces, L.B., Bergink, S., Marteijn, J.A., Geverts, B., Houtsmuller, A.B., Vermeulen, W., Di Fiore, P.P., et al. (2007). Human USP3 is a chromatin modifier required for $S$ phase progression and genome stability. Curr. Biol. CB 17, 1972-1977.

Ogba, N., Chaplin, L.J., Doughman, Y.Q., Fujinaga, K., and Montano, M.M. (2008). HEXIM1

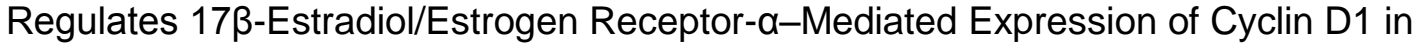
Mammary Cells via Modulation of P-TEFb. Cancer Res. 68, 7015-7024.

Ong, C.-T., and Corces, V.G. (2011). Enhancer function: new insights into the regulation of tissue-specific gene expression. Nat. Rev. Genet. 12, 283-293. 
Orlando, V. (2003). Polycomb, epigenomes, and control of cell identity. Cell 112, 599-606.

Pan, G., Tian, S., Nie, J., Yang, C., Ruotti, V., Wei, H., Jonsdottir, G.A., Stewart, R., and Thomson, J.A. (2007). Whole-Genome Analysis of Histone H3 Lysine 4 and Lysine 27 Methylation in Human Embryonic Stem Cells. Cell Stem Cell 1, 299-312.

Park, S.-M., Gaur, A.B., Lengyel, E., and Peter, M.E. (2008). The miR-200 family determines the epithelial phenotype of cancer cells by targeting the E-cadherin repressors ZEB1 and ZEB2. Genes Dev. 22, 894-907.

Parker, M.G. (1995). Structure and function of estrogen receptors. Vitam. Horm. 51, 267287.

Pavri, R., Zhu, B., Li, G., Trojer, P., Mandal, S., Shilatifard, A., and Reinberg, D. (2006). Histone H2B Monoubiquitination Functions Cooperatively with FACT to Regulate Elongation by RNA Polymerase II. Cell 125, 703-717.

Pece, S., Tosoni, D., Confalonieri, S., Mazzarol, G., Vecchi, M., Ronzoni, S., Bernard, L., Viale, G., Pelicci, P.G., and Di Fiore, P.P. (2010). Biological and molecular heterogeneity of breast cancers correlates with their cancer stem cell content. Cell 140, 62-73.

Pedersen, M.W., Pedersen, N., Damstrup, L., Villingshøj, M., Sønder, S.U., Rieneck, K., Bovin, L.F., Spang-Thomsen, M., and Poulsen, H.S. (2005). Analysis of the epidermal growth factor receptor specific transcriptome: Effect of receptor expression level and an activating mutation. J. Cell. Biochem. 96, 412-427.

Peinado, H., Lavotshkin, S., and Lyden, D. (2011). The secreted factors responsible for premetastatic niche formation: Old sayings and new thoughts. Semin. Cancer Biol. 21, 139146.

Peña, C., García, J.M., García, V., Silva, J., Domínguez, G., Rodríguez, R., Maximiano, C., García de Herreros, A., Muñoz, A., and Bonilla, F. (2006). The expression levels of the transcriptional regulators p300 and CtBP modulate the correlations between SNAIL, ZEB1, E-cadherin and vitamin D receptor in human colon carcinomas. Int. J. Cancer 119, 20982104.

Peng, J., Zhu, Y., Milton, J.T., and Price, D.H. (1998). Identification of multiple cyclin subunits of human P-TEFb. Genes Dev. 12, 755-762.

Peterlin, B.M., and Price, D.H. (2006). Controlling the Elongation Phase of Transcription with P-TEFb. Mol. Cell 23, 297-305.

Peterson, C.L., and Tamkun, J.W. (1995). The SWI-SNF complex: a chromatin remodeling machine? Trends Biochem. Sci. 20, 143-146.

Pettersson, K., and Gustafsson, J.-Å. (2001). Role of Estrogen Receptor Beta in Estrogen Action. Annu. Rev. Physiol. 63, 165-192.

Pham, C.T.N., Maclvor, D.M., Hug, B.A., Heusel, J.W., and Ley, T.J. (1996). Long-range disruption of gene expression by a selectable marker cassette. Proc. Natl. Acad. Sci. 93, 13090-13095.

Pirngruber, J., Shchebet, A., Schreiber, L., Shema, E., Minsky, N., Chapman, R.D., Eick, D., Aylon, Y., Oren, M., and Johnsen, S.A. (2009a). CDK9 directs H2B monoubiquitination and controls replication-dependent histone mRNA 3'-end processing. EMBO Rep. 10, 894-900. 
Pirngruber, J., Shchebet, A., and Johnsen, S.A. (2009b). Insights into the function of the human P-TEFb component CDK9 in the regulation of chromatin modifications and cotranscriptional mRNA processing. Cell Cycle 8, 3636-3642.

Pirngruber, J., Shchebet, A., and Johnsen, S.A. Insights into the function of the human PTEFb component CDK9 in the regulation of chromatin modifications and co-transcriptional mRNA processing. Cell Cycle 8, 3636-3642.

Polyak, K., and Weinberg, R.A. (2009). Transitions between epithelial and mesenchymal states: acquisition of malignant and stem cell traits. Nat. Rev. Cancer 9, 265-273.

Ponti, D., Costa, A., Zaffaroni, N., Pratesi, G., Petrangolini, G., Coradini, D., Pilotti, S., Pierotti, M.A., and Daidone, M.G. (2005). Isolation and In vitro Propagation of Tumorigenic Breast Cancer Cells with Stem/Progenitor Cell Properties. Cancer Res. 65, 5506-5511.

Prenzel, T., Begus-Nahrmann, Y., Kramer, F., Hennion, M., Hsu, C., Gorsler, T., Hintermair, C., Eick, D., Kremmer, E., Simons, M., et al. (2011). Estrogen-dependent gene transcription in human breast cancer cells relies upon proteasome-dependent monoubiquitination of histone H2B. Cancer Res. 71, 5739-5753.

Qin, L., Liu, Z., Chen, H., and Xu, J. (2009). The Steroid Receptor Coactivator-1 Regulates Twist Expression and Promotes Breast Cancer Metastasis. Cancer Res. 69, 3819-3827.

Raaphorst, F.M., Meijer, C.J.L.M., Fieret, E., Blokzijl, T., Mommers, E., Buerger, H., Packeisen, J., Sewalt, R.A.B., Otte, A.P., and van Diest, P.J. (2003). Poorly differentiated breast carcinoma is associated with increased expression of the human polycomb group EZH2 gene. Neoplasia N. Y. N 5, 481-488.

Radhakrishnan, S.K., and Gartel, A.L. CDK9 Phosphorylates p53 on Serine Residues 33, 315 and 392. Cell Cycle 5, 519-521.

Ramadoss, S., Chen, X., and Wang, C.-Y. (2012). Histone Demethylase KDM6B Promotes Epithelial-Mesenchymal Transition. J. Biol. Chem. 287, 44508-44517.

Reijm, E.A., Jansen, M.P.H.M., Ruigrok-Ritstier, K., van Staveren, I.L., Look, M.P., van Gelder, M.E.M., Sieuwerts, A.M., Sleijfer, S., Foekens, J.A., and Berns, E.M.J.J. (2011). Decreased expression of EZH2 is associated with upregulation of ER and favorable outcome to tamoxifen in advanced breast cancer. Breast Cancer Res. Treat. 125, 387-394.

Ren, S.-Y., Angrand, P.-O., and Rijli, F.M. (2002). Targeted insertion results in a Rhombomere 2-specific Hoxa2 knockdown and ectopic activation of Hoxa1 expression. Dev. Dyn. 225, 305-315.

Reya, T., Morrison, S.J., Clarke, M.F., and Weissman, I.L. (2001). Stem cells, cancer, and cancer stem cells. Nature 414, 105-111.

Richardson, A.L., Wang, Z.C., De Nicolo, A., Lu, X., Brown, M., Miron, A., Liao, X., Iglehart, J.D., Livingston, D.M., and Ganesan, S. (2006). X chromosomal abnormalities in basal-like human breast cancer. Cancer Cell 9, 121-132.

Richmond, T.J., Finch, J.T., Rushton, B., Rhodes, D., and Klug, A. (1984). Structure of the nucleosome core particle at 7 A resolution. Nature 311, 532-537.

Ringrose, L., and Paro, R. (2004). Epigenetic regulation of cellular memory by the Polycomb and Trithorax group proteins. Annu. Rev. Genet. 38, 413-443. 
Roche, J., Nasarre, P., Gemmill, R., Baldys, A., Pontis, J., Korch, C., Guilhot, J., Ait-Si-Ali, S., and Drabkin, H. (2013). Global Decrease of Histone H3K27 Acetylation in ZEB1-Induced Epithelial to Mesenchymal Transition in Lung Cancer Cells. Cancers 5, 334-356.

Ruifrok, A.C., and Johnston, D.A. (2001). Quantification of histochemical staining by color deconvolution. Anal. Quant. Cytol. Histol. Int. Acad. Cytol. Am. Soc. Cytol. 23, 291-299.

Saatcioglu, F., Bartunek, P., Deng, T., Zenke, M., and Karin, M. (1993). A conserved Cterminal sequence that is deleted in $\mathrm{v}$-ErbA is essential for the biological activities of c-ErbA (the thyroid hormone receptor). Mol. Cell. Biol. 13, 3675-3685.

Sánchez-Tilló, E., Lázaro, A., Torrent, R., Cuatrecasas, M., Vaquero, E.C., Castells, A., Engel, P., and Postigo, A. (2010). ZEB1 represses E-cadherin and induces an EMT by recruiting the SWI/SNF chromatin-remodeling protein BRG1. Oncogene 29, 3490-3500.

Sarrió, D., Rodriguez-Pinilla, S.M., Hardisson, D., Cano, A., Moreno-Bueno, G., and Palacios, J. (2008). Epithelial-mesenchymal transition in breast cancer relates to the basallike phenotype. Cancer Res. 68, 989-997.

Scacheri, P. c., Crabtree, J. s., Novotny, E. a., Garrett-Beal, L., Chen, A., Edgemon, K. a., Marx, S. j., Spiegel, A. m., Chandrasekharappa, S. c., and Collins, F. s. (2001). Bidirectional transcriptional activity of PGK-neomycin and unexpected embryonic lethality in heterozygote chimeric knockout mice. Genesis 30, 259-263.

Scheel, C., and Weinberg, R.A. (2012). Cancer stem cells and epithelial-mesenchymal transition: Concepts and molecular links. Semin. Cancer Biol. 22, 396-403.

Schneider, C.A., Rasband, W.S., and Eliceiri, K.W. (2012). NIH Image to ImageJ: 25 years of image analysis. Nat. Methods 9, 671-675.

Schuettengruber, B., Chourrout, D., Vervoort, M., Leblanc, B., and Cavalli, G. (2007). Genome Regulation by Polycomb and Trithorax Proteins. Cell 128, 735-745.

Schwabish, M.A., and Struhl, K. (2004). Evidence for Eviction and Rapid Deposition of Histones upon Transcriptional Elongation by RNA Polymerase II. Mol. Cell. Biol. 24, 1011110117.

Seenundun, S., Rampalli, S., Liu, Q.-C., Aziz, A., Palii, C., Hong, S., Blais, A., Brand, M., Ge, K., and Dilworth, F.J. (2010). UTX mediates demethylation of H3K27me3 at musclespecific genes during myogenesis. EMBO J. 29, 1401-1411.

Shan, B., Zhuo, Y., Chin, D., Morris, C.A., Morris, G.F., and Lasky, J.A. (2005). Cyclindependent Kinase 9 Is Required for Tumor Necrosis Factor- $\alpha$-stimulated Matrix Metalloproteinase-9 Expression in Human Lung Adenocarcinoma Cells. J. Biol. Chem. 280, 1103-1111.

Shema, E., Tirosh, I., Aylon, Y., Huang, J., Ye, C., Moskovits, N., Raver-Shapira, N., Minsky, N., Pirngruber, J., Tarcic, G., et al. (2008a). The histone H2B-specific ubiquitin ligase RNF20/hBRE1 acts as a putative tumor suppressor through selective regulation of gene expression. Genes Dev. 22, 2664-2676.

Shema, E., Tirosh, I., Aylon, Y., Huang, J., Ye, C., Moskovits, N., Raver-Shapira, N., Minsky, N., Pirngruber, J., Tarcic, G., et al. (2008b). The histone H2B-specific ubiquitin ligase RNF20/hBRE1 acts as a putative tumor suppressor through selective regulation of gene expression. Genes Dev. 22, 2664-2676. 
Shi, Y., Lan, F., Matson, C., Mulligan, P., Whetstine, J.R., Cole, P.A., Casero, R.A., and Shi, Y. (2004). Histone demethylation mediated by the nuclear amine oxidase homolog LSD1. Cell 119, 941-953.

Shimono, Y., Zabala, M., Cho, R.W., Lobo, N., Dalerba, P., Qian, D., Diehn, M., Liu, H., Panula, S.P., Chiao, E., et al. (2009). Downregulation of miRNA-200c links breast cancer stem cells with normal stem cells. Cell 138, 592-603.

Shukla, A., Stanojevic, N., Duan, Z., Shadle, T., and Bhaumik, S.R. (2006). Functional analysis of H2B-Lys-123 ubiquitination in regulation of H3-Lys-4 methylation and recruitment of RNA polymerase II at the coding sequences of several active genes in vivo. J. Biol. Chem. 281, 19045-19054.

Sif, S. (2004). ATP-dependent nucleosome remodeling complexes: Enzymes tailored to deal with chromatin. J. Cell. Biochem. 91, 1087-1098.

Simone, C., and Giordano, A. (2006). Abrogation of signal-dependent activation of the cdk9/cyclin T2a complex in human RD rhabdomyosarcoma cells. Cell Death Differ. 14, 192195.

Simonsen, J.L., Rosada, C., Serakinci, N., Justesen, J., Stenderup, K., Rattan, S.I.S., Jensen, T.G., and Kassem, M. (2002). Telomerase expression extends the proliferative lifespan and maintains the osteogenic potential of human bone marrow stromal cells. Nat. Biotechnol. 20, 592-596.

Smith, S., and Stillman, B. (1991). Stepwise assembly of chromatin during DNA replication in vitro. EMBO J. 10, 971-980.

Song, L.-B., Li, J., Liao, W.-T., Feng, Y., Yu, C.-P., Hu, L.-J., Kong, Q.-L., Xu, L.-H., Zhang, X., Liu, W.-L., et al. (2009). The polycomb group protein Bmi-1 represses the tumor suppressor PTEN and induces epithelial-mesenchymal transition in human nasopharyngeal epithelial cells. J. Clin. Invest. 119, 3626-3636.

Song, S.J., Ito, K., Ala, U., Kats, L., Webster, K., Sun, S.M., Jongen-Lavrencic, M., ManovaTodorova, K., Teruya-Feldstein, J., Avigan, D.E., et al. (2013). The Oncogenic MicroRNA miR-22 Targets the TET2 Tumor Suppressor to Promote Hematopoietic Stem Cell SelfRenewal and Transformation. Cell Stem Cell 13, 87-101.

Sørlie, T., Perou, C.M., Tibshirani, R., Aas, T., Geisler, S., Johnsen, H., Hastie, T., Eisen, M.B., van de Rijn, M., Jeffrey, S.S., et al. (2001). Gene expression patterns of breast carcinomas distinguish tumor subclasses with clinical implications. Proc. Natl. Acad. Sci. U. S. A. $98,10869-10874$.

Spaderna, S., Schmalhofer, O., Wahlbuhl, M., Dimmler, A., Bauer, K., Sultan, A., Hlubek, F., Jung, A., Strand, D., Eger, A., et al. (2008). The transcriptional repressor ZEB1 promotes metastasis and loss of cell polarity in cancer. Cancer Res. 68, 537-544.

Stokes, M.P., Rush, J., MacNeill, J., Ren, J.M., Sprott, K., Nardone, J., Yang, V., Beausoleil, S.A., Gygi, S.P., Livingstone, M., et al. (2007). Profiling of UV-induced ATM/ATR signaling pathways. Proc. Natl. Acad. Sci. 104, 19855-19860.

Tang, M., Shen, H., Jin, Y., Lin, T., Cai, Q., Pinard, M.A., Biswas, S., Tran, Q., Li, G., Shenoy, A.K., et al. (2013). The Malignant Brain Tumor (MBT) Domain Protein SFMBT1 Is an Integral Histone Reader Subunit of the LSD1 Demethylase Complex for Chromatin Association and Epithelial-to-mesenchymal Transition. J. Biol. Chem. 288, 27680-27691. 
Tepass, U., Truong, K., Godt, D., Ikura, M., and Peifer, M. (2000). Cadherins in embryonic and neural morphogenesis. Nat. Rev. Mol. Cell Biol. 1, 91-100.

Theodorou, V., Stark, R., Menon, S., and Carroll, J.S. (2013). GATA3 acts upstream of FOXA1 in mediating ESR1 binding by shaping enhancer accessibility. Genome Res. 23, 12 22.

Tiwari, N., Tiwari, V.K., Waldmeier, L., Balwierz, P.J., Arnold, P., Pachkov, M., MeyerSchaller, N., Schübeler, D., van Nimwegen, E., and Christofori, G. (2013). Sox4 is a master regulator of epithelial-mesenchymal transition by controlling Ezh2 expression and epigenetic reprogramming. Cancer Cell 23, 768-783.

Tora, L., White, J., Brou, C., Tasset, D., Webster, N., Scheer, E., and Chambon, P. (1989). The human estrogen receptor has two independent nonacidic transcriptional activation functions. Cell 59, 477-487.

Towbin, H., Staehelin, T., and Gordon, J. (1979). Electrophoretic transfer of proteins from polyacrylamide gels to nitrocellulose sheets: procedure and some applications. Proc. Natl. Acad. Sci. U. S. A. 76, 4350-4354.

Tsai, M., and O'Malley, B.W. (1994). Molecular Mechanisms of Action of Steroid/Thyroid Receptor Superfamily Members. Annu. Rev. Biochem. 63, 451-486.

Urasaki, Y., Heath, L., and Xu, C.W. (2012). Coupling of Glucose Deprivation with Impaired Histone H2B Monoubiquitination in Tumors. PLoS ONE 7, e36775.

Vandewalle, C., Comijn, J., De Craene, B., Vermassen, P., Bruyneel, E., Andersen, H., Tulchinsky, E., Van Roy, F., and Berx, G. (2005). SIP1/ZEB2 induces EMT by repressing genes of different epithelial cell-cell junctions. Nucleic Acids Res. 33, 6566-6578.

Wagner, K.-U., Wall, R.J., St-Onge, L., Gruss, P., Wynshaw-Boris, A., Garrett, L., Li, M., Furth, P.A., and Hennighausen, L. (1997). Cre-mediated gene deletion in the mammary gland. Nucleic Acids Res. 25, 4323-4330.

Wang, Y., and Shang, Y. (2013). Epigenetic control of epithelial-to-mesenchymal transition and cancer metastasis. Exp. Cell Res. 319, 160-169.

Wang, A.H., Zare, H., Mousavi, K., Wang, C., Moravec, C.E., Sirotkin, H.I., Ge, K., Gutierrez-Cruz, G., and Sartorelli, V. (2013a). The histone chaperone Spt6 coordinates histone H3K27 demethylation and myogenesis. EMBO J. 32, 1075-1086.

Wang, A.H., Zare, H., Mousavi, K., Wang, C., Moravec, C.E., Sirotkin, H.I., Ge, K., Gutierrez-Cruz, G., and Sartorelli, V. (2013b). The histone chaperone Spt6 coordinates histone H3K27 demethylation and myogenesis. EMBO J. 32, 1075-1086.

Wang, H., Wang, L., Erdjument-Bromage, H., Vidal, M., Tempst, P., Jones, R.S., and Zhang, Y. (2004). Role of histone H2A ubiquitination in Polycomb silencing. Nature 431, 873-878.

Wellner, U., Schubert, J., Burk, U.C., Schmalhofer, O., Zhu, F., Sonntag, A., Waldvogel, B., Vannier, C., Darling, D., Hausen, A. zur, et al. (2009a). The EMT-activator ZEB1 promotes tumorigenicity by repressing stemness-inhibiting microRNAs. Nat. Cell Biol. 11, 1487-1495.

Wellner, U., Schubert, J., Burk, U.C., Schmalhofer, O., Zhu, F., Sonntag, A., Waldvogel, B., Vannier, C., Darling, D., Hausen, A. zur, et al. (2009b). The EMT-activator ZEB1 promotes tumorigenicity by repressing stemness-inhibiting microRNAs. Nat. Cell Biol. 11, 1487-1495. 
White, R., and Parker, M.G. (1998). Molecular mechanisms of steroid hormone action. Endocr. Relat. Cancer 5, 1-14.

Wittmann, B.M., Fujinaga, K., Deng, H., Ogba, N., and Montano, M.M. (2005). The breast cell growth inhibitor, estrogen down regulated gene 1, modulates a novel functional interaction between estrogen receptor alpha and transcriptional elongation factor cyclin T1. Oncogene 24, 5576-5588.

Wong, A.K.C., Shanahan, F., Chen, Y., Lian, L., Ha, P., Hendricks, K., Ghaffari, S., Iliev, D., Penn, B., Woodland, A.-M., et al. (2000). BRG1, a Component of the SWI-SNF Complex, Is Mutated in Multiple Human Tumor Cell Lines. Cancer Res. 60, 6171-6177.

Wood, A., Krogan, N.J., Dover, J., Schneider, J., Heidt, J., Boateng, M.A., Dean, K., Golshani, A., Zhang, Y., Greenblatt, J.F., et al. (2003). Bre1, an E3 Ubiquitin Ligase Required for Recruitment and Substrate Selection of Rad6 at a Promoter. Mol. Cell 11, 267274.

Wu, S.-Y., and Chiang, C.-M. (2007a). The double bromodomain-containing chromatin adaptor Brd4 and transcriptional regulation. J. Biol. Chem. 282, 13141-13145.

Wu, S.-Y., and Chiang, C.-M. (2007b). The Double Bromodomain-containing Chromatin Adaptor Brd4 and Transcriptional Regulation. J. Biol. Chem. 282, 13141-13145.

Wu, M.-Z., Tsai, Y.-P., Yang, M.-H., Huang, C.-H., Chang, S.-Y., Chang, C.-C., Teng, S.-C., and Wu, K.-J. (2011a). Interplay between HDAC3 and WDR5 Is Essential for HypoxiaInduced Epithelial-Mesenchymal Transition. Mol. Cell 43, 811-822.

Wu, M.-Z., Tsai, Y.-P., Yang, M.-H., Huang, C.-H., Chang, S.-Y., Chang, C.-C., Teng, S.-C., and Wu, K.-J. (2011b). Interplay between HDAC3 and WDR5 is essential for hypoxiainduced epithelial-mesenchymal transition. Mol. Cell 43, 811-822.

Wu, S.-Y., Lee, A.-Y., Hou, S.Y., Kemper, J.K., Erdjument-Bromage, H., Tempst, P., and Chiang, C.-M. (2006). Brd4 links chromatin targeting to HPV transcriptional silencing. Genes Dev. 20, 2383-2396.

Xiao, T., Kao, C.-F., Krogan, N.J., Sun, Z.-W., Greenblatt, J.F., Osley, M.A., and Strahl, B.D. (2005). Histone H2B Ubiquitylation Is Associated with Elongating RNA Polymerase II. Mol. Cell. Biol. 25, 637-651.

Yamada, T., Yamaguchi, Y., Inukai, N., Okamoto, S., Mura, T., and Handa, H. (2006). PTEFb-Mediated Phosphorylation of hSpt5 C-Terminal Repeats Is Critical for Processive Transcription Elongation. Mol. Cell 21, 227-237.

Yamaguchi, Y., Narita, T., Inukai, N., Wada, T., and Handa, H. (2001). SPT Genes: Key Players in the Regulation of Transcription, Chromatin Structure and Other Cellular Processes. J. Biochem. (Tokyo) 129, 185-191.

Yang, H., Liu, Y., Bai, F., Zhang, J.-Y., Ma, S.-H., Liu, J., Xu, Z.-D., Zhu, H.-G., Ling, Z.-Q., Ye, D., et al. (2013). Tumor development is associated with decrease of TET gene expression and 5-methylcytosine hydroxylation. Oncogene 32, 663-669.

Yang, M.-H., Hsu, D.S.-S., Wang, H.-W., Wang, H.-J., Lan, H.-Y., Yang, W.-H., Huang, C.H., Kao, S.-Y., Tzeng, C.-H., Tai, S.-K., et al. (2010). Bmi1 is essential in Twist1-induced epithelial-mesenchymal transition. Nat. Cell Biol. 12, 982-992. 
Yang, Z., Yik, J.H.N., Chen, R., He, N., Jang, M.K., Ozato, K., and Zhou, Q. (2005). Recruitment of P-TEFb for Stimulation of Transcriptional Elongation by the Bromodomain Protein Brd4. Mol. Cell 19, 535-545.

Yap, K.L., and Zhou, M.-M. (2011). Structure and Mechanisms of Lysine Methylation Recognition by the Chromodomain in Gene Transcription. Biochemistry (Mosc.) 50, 19661980.

Yik, J.H.., Chen, R., Nishimura, R., Jennings, J.L., Link, A.J., and Zhou, Q. (2003). Inhibition of P-TEFb (CDK9/Cyclin T) Kinase and RNA Polymerase II Transcription by the Coordinated Actions of HEXIM1 and 7SK snRNA. Mol. Cell 12, 971-982.

Yoh, S.M., Cho, H., Pickle, L., Evans, R.M., and Jones, K.A. (2007). The Spt6 SH2 domain binds Ser2-P RNAPII to direct Iws1-dependent mRNA splicing and export. Genes Dev. 21, 160-174.

Yoshikawa, M., Hishikawa, K., Marumo, T., and Fujita, T. (2007). Inhibition of Histone Deacetylase Activity Suppresses Epithelial-to-Mesenchymal Transition Induced by TGF- $\beta 1$ in Human Renal Epithelial Cells. J. Am. Soc. Nephrol. 18, 58-65.

Zajicek, G. (1996). A new cancer hypothesis. Med. Hypotheses 47, 111-115.

Zhang, F., and Yu, X. (2011a). WAC, a Functional Partner of RNF20/40, Regulates Histone H2B Ubiquitination and Gene Transcription. Mol. Cell 41, 384-397.

Zhang, F., and Yu, X. (2011b). WAC, a Functional Partner of RNF20/40, Regulates Histone H2B Ubiquitination and Gene Transcription. Mol. Cell 41, 384-397.

Zhang, X.-Y., Varthi, M., Sykes, S.M., Phillips, C., Warzecha, C., Zhu, W., Wyce, A., Thorne, A.W., Berger, S.L., and McMahon, S.B. (2008a). The Putative Cancer Stem Cell Marker USP22 Is a Subunit of the Human SAGA Complex Required for Activated Transcription and Cell-Cycle Progression. Mol. Cell 29, 102-111.

Zhang, X.-Y., Varthi, M., Sykes, S.M., Phillips, C., Warzecha, C., Zhu, W., Wyce, A., Thorne, A.W., Berger, S.L., and McMahon, S.B. (2008b). The Putative Cancer Stem Cell Marker USP22 Is a Subunit of the Human SAGA Complex Required for Activated Transcription and Cell-Cycle Progression. Mol. Cell 29, 102-111.

Zhao, L., Li, W., Zang, W., Liu, Z., Xu, X., Yu, H., Yang, Q., and Jia, J. (2013). JMJD2B promotes epithelial-mesenchymal transition by cooperating with $\beta$-catenin and enhances gastric cancer metastasis. Clin. Cancer Res. clincanres.0254.2013.

Zhao, X.D., Han, X., Chew, J.L., Liu, J., Chiu, K.P., Choo, A., Orlov, Y.L., Sung, W.-K., Shahab, A., Kuznetsov, V.A., et al. (2007). Whole-Genome Mapping of Histone H3 Lys4 and 27 Trimethylations Reveals Distinct Genomic Compartments in Human Embryonic Stem Cells. Cell Stem Cell 1, 286-298.

Zhao, Y., Lang, G., Ito, S., Bonnet, J., Metzger, E., Sawatsubashi, S., Suzuki, E., Le Guezennec, X., Stunnenberg, H.G., Krasnov, A., et al. (2008). A TFTC/STAGA Module Mediates Histone H2A and H2B Deubiquitination, Coactivates Nuclear Receptors, and Counteracts Heterochromatin Silencing. Mol. Cell 29, 92-101.

Zhou, H.-J., Yan, J., Luo, W., Ayala, G., Lin, S.-H., Erdem, H., Ittmann, M., Tsai, S.Y., and Tsai, M.-J. (2005). SRC-3 Is Required for Prostate Cancer Cell Proliferation and Survival. Cancer Res. 65, 7976-7983. 
Zhou, M., Huang, K., Jung, K.-J., Cho, W.-K., Klase, Z., Kashanchi, F., Pise-Masison, C.A., and Brady, J.N. (2009). Bromodomain Protein Brd4 Regulates Human Immunodeficiency Virus Transcription through Phosphorylation of CDK9 at Threonine 29. J. Virol. 83, 10361044.

Zhu, B., Zheng, Y., Pham, A.-D., Mandal, S.S., Erdjument-Bromage, H., Tempst, P., and Reinberg, D. (2005a). Monoubiquitination of Human Histone H2B: The Factors Involved and Their Roles in HOX Gene Regulation. Mol. Cell 20, 601-611.

Zhu, B., Zheng, Y., Pham, A.-D., Mandal, S.S., Erdjument-Bromage, H., Tempst, P., and Reinberg, D. (2005b). Monoubiquitination of Human Histone H2B: The Factors Involved and Their Roles in HOX Gene Regulation. Mol. Cell 20, 601-611.

Zuber, J., Shi, J., Wang, E., Rappaport, A.R., Herrmann, H., Sison, E.A., Magoon, D., Qi, J., Blatt, K., Wunderlich, M., et al. (2011a). RNAi screen identifies Brd4 as a therapeutic target in acute myeloid leukaemia. Nature 478, 524-528.

Zuber, J., Shi, J., Wang, E., Rappaport, A.R., Herrmann, H., Sison, E.A., Magoon, D., Qi, J., Blatt, K., Wunderlich, M., et al. (2011b). RNAi screen identifies Brd4 as a therapeutic target in acute myeloid leukaemia. Nature 478, 524-528. 